\author{
Monograph \\ urn:lsid:zoobank.org:pub:173B2F6E-D172-41FE-A78D-FDCE5562E1BB
}

\title{
Review of Aphidiinae parasitoids (Hymenoptera: Braconidae) of the Middle East and North Africa: key to species and host associations
}

\author{
Ehsan RAKHSHANI ${ }^{1, *}$, Hossein BARAHOEI ${ }^{2}$, Zubair AHMAD $^{3}$, Petr STARÝ ${ }^{4}$, \\ Mostafa GHAFOURI-MOGHADDAM ${ }^{5}$, Mohsen MEHRPARVAR ${ }^{6}$, \\ Nickolas G. KAVALLIERATOS ${ }^{7}$, Jelisaveta $\breve{C K R K I C ́}^{8} \& \breve{Z ̌ e l j k o ~ T O M A N O V I C ́ ~}^{9}$ \\ ${ }^{1}$ Department of Plant Protection, College of Agriculture, \\ University of Zabol, P.O. Box 98615-538, I.R. Iran. \\ ${ }^{2}$ Institute of Agricultural Research, University of Zabol, P.O. Box 98615-538, I.R. Iran. \\ ${ }^{3}$ Research Center Advanced Materials Science (RCAMS), King Khalid University, \\ 9004, Abha 61413, Saudi Arabia. \\ ${ }^{3}$ Unit of Bee Research and Honey Production, Faculty of Science, King Khalid University, \\ P.O. Box 9004, Abha 61413, Saudi Arabia. \\ ${ }^{3}$ Department of Biology, Faculty of Sciences and Arts, King Khalid University, \\ Dhahran Al Janoub, Saudi Arabia. \\ ${ }^{4}$ Institute of Entomology, Biology Center, Laboratory of Aphidology, AVCR, \\ Branišovská 31, 37005 České Budějovice, Czech Republic. \\ ${ }^{5}$ Department of Plant Protection, College of Agriculture, \\ University of Zabol, P.O. Box 98615-538, I.R. Iran. \\ ${ }^{6}$ Department of Biodiversity, Institute of Science and High Technology and Environmental Sciences, \\ Graduate University of Advanced Technology, Kerman, I.R. Iran. \\ ${ }^{7}$ Laboratory of Agricultural Zoology and Entomology, Department of Crop Science, Agricultural \\ University of Athens, 75 Iera Odos str., 11855, Athens, Attica, Greece. \\ ${ }^{8,9}$ Institute of Zoology, Faculty of Biology, University of Belgrade, \\ Studensky trg 16, 11000 Belgrade, Serbia. \\ *Corresponding author: rakhshani@uoz.ac.ir \\ ${ }^{2}$ Email: hossein.barahoei@gmail.com \\ 32Email: dzubair@gmail.com \\ ${ }^{4}$ Email: stary@entu.cas.cz \\ ${ }^{5}$ Email: msc.ghafouri@gmail.com \\ ${ }^{6}$ Email: mehrparvar@aphidology.com \\ ${ }^{7}$ Email: nick_kaval@hotmail.com \\ ${ }^{8}$ Email: jckrkic@bio.bg.ac.rs \\ ${ }^{9}$ Email: ztoman@bio.bg.ac.rs

\footnotetext{
${ }^{1}$ urn:lsid:zoobank.org:author:86C5B3A2-BE3E-4C3B-B0F1-3B69148E0B9B

${ }^{2}$ urn:1sid:zoobank.org:author:44789571-73AB-4B58-ADA3-AF43B9EAC4BA

${ }^{3}$ urn:Isid:zoobank.org:author:769585C9-7C8C-4381-9DFE-ED5C32EFDCEE

${ }^{4}$ urn:1sid:zoobank.org:author:91D5DFA9-E28A-4DCA-A6A0-D05E125BD22D

${ }^{5}$ urn:1sid:zoobank.org:author:55BDC589-8306-4346-8712-7CB6F64BDA00
} 


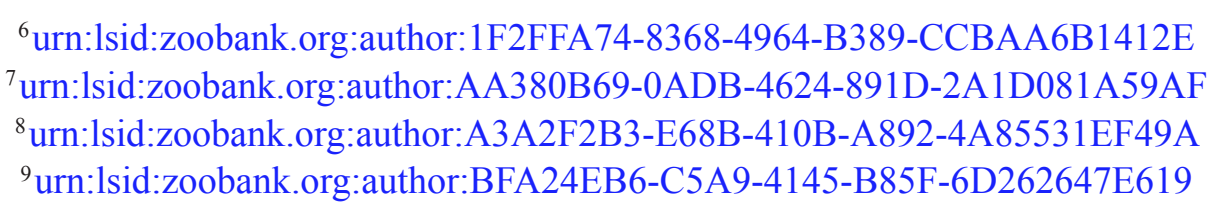

\begin{abstract}
In this study, a total of 108 Aphidiinae species, belonging to 18 genera, associated with 240 aphid species in 16 countries of the Middle East and North Africa are reviewed. 743 host aphidparasitoid associations are listed. New material was collected from various regions of Saudi Arabia during 2011-2013. Three species including Aphidius avenae Haliday, 1834, Aphidius platensis Brèthes, 1913 and Praon barbatum Mackauer, 1967 are first recorded for the fauna of this country. Lysiphlebus marismortui Mescheloff \& Rosen, 1990 syn. nov. is classified as the junior synonym of Lysiphlebus confusus Tremblay \& Eady, 1978. An illustrated up-to-date key to all known species of Aphidiinae that occur in the Middle East and North Africa is provided. The findings are discussed in relation to the overall parasitoid-aphid associations in the target investigated region.
\end{abstract}

Keywords. Aphids, parasitoids, fauna, Western Asia, North Africa, illustrated key.

Rakhshani E., Barahoei H., Ahmad Z., Starý P., Ghafouri-Moghaddam M., Mehrparvar M., Kavallieratos N.G., Čkrkić J. \& Tomanović Z. 2019. Review of Aphidiinae parasitoids (Hymenoptera: Braconidae) of the Middle East and North Africa: key to species and host associations. European Journal of Taxonomy 552: 1-132. https://doi.org/10.5852/ejt.2019.552

\title{
Introduction
}

Members of the subfamily Aphidiinae (Hymenoptera: Braconidae) belong to an important group as natural enemies of aphids (Starý 1970). Aphidiinae consist of solitary koinobiont parasitoids, among which several species are considered highly efficient biological control agents (Hågvar \& Hofsvang 1991; Boivin et al. 2012). This subfamily includes about 505 species belonging to 38 genera, around the world (Žikić et al. 2017). Aphidiinae are strictly associated to their host aphids, regarding local distribution and/or broader expansions (Starý 1981a). The complexity of biogeographical factors determines the area of distribution of parasitoid species and directly affects the host associations (Hawkins 1994). Consequently, each aphid parasitoid species is also expected to have a specific territory from which it can be distributed and reach the preferable host aphids (Charles \& Paine 2016). Additionally, the host plants have a crucial impact concerning the diversity of the feeding insects, especially on aphids and their parasitoids (Starý 1981a; Žikić et al. 2017).

The Middle East is a region that encompasses Western Asia and a part of North Africa. The traditional definition of the Middle East includes counties in Western Asia (Bahrain, Iran, Iraq, Israel, Jordan, Kuwait, Lebanon, Oman, Palestine, Saudi Arabia, Syria, United Arab Emirates and Yemen). Yet, the "greater" Middle East includes many other countries in central Asia (Afghanistan, Armenia, Azerbaijan, Georgia, Kazakhstan, Kyrgyzstan, Pakistan, Tajikistan, Turkmenistan, Uzbekistan, Turkey) and some northern African countries (Algeria, Egypt, Libya, Morocco, Somalia, Sudan and Tunisia). However, regardless of the geographical definition, a delimitation of the area is more biogeographically substantial with few additions of other countries including Turkey and Mediterranean North African countries. The countries of the Middle East generally have an arid and hot climate, relying on several major rivers to provide water for irrigation in order to support agriculture in moderately limited areas. The biotic history of Asia, as well as its complex topography and climatic diversity, led to the evolution of thousands of very specific plant species. The natural barrier of the Irano-Anatolian area, which is located between the Mediterranean Basin and the dry plateaus of Western Asia, serves as both a refuge and a corridor and 
it has contributed to the creation of many isolated areas with various patches of local endemism (Starý et al. 2000; Ma et al. 2010).

Such a complicated diversity of plant species, which is affected partially by the agricultural intensification, needs to be highlighted in conservation programs. The analyses of the faunal diversity and the biological connectivity of the regions, in the case of aphid parasitoids, are the main topics of ecological and conservation studies (Powell et al. 1990; Vollhardt et al. 2008). The area of North African countries is biogeographically divided into the Mediterranean climate region in the north, and the arid Sahara in the south, albeit both of them can be subdivided into various ecozones (Houerou 2000). Numerous faunistic and biosystematical research studies on Aphidiinae have been conducted in this complex region, although many parts are still unexplored. Earlier studies on aphid parasitoids of Central Asia (Starý 1979) and the Mediterranean (Starý 1976) include also some parts of the Middle East. Furthermore, scattered information has been published, in which the occurrence of the aphid parasitoid species has been recorded in various countries in the Middle East and North Africa.

The few published identification keys of Aphidiinae are restricted to the local fauna of Aphidiinae (i.e., Iran) (Nazari et al. 2012; Rakhshani et al. 2012b; Barahoei et al. 2013), local generic keys (Mescheloff \& Rosen 1988, 1990a, 1990b, 1993; Rakhshani et al. 2007a, 2008a, 2012a) or specific groups associated with economically important aphids (Rakhshani et al. 2005a, 2006a; Rakhshani 2012; Gadallah et al. 2017). However, these keys are associated with few countries in the Middle East, while numerous species have never been included. Here we review the Aphidiinae parasitoids of the Middle East and North Africa, aiming to compare their diversity in various countries and establish a background for future research, especially within the unexplored areas. New material, originating from Saudi Arabia, has been studied and new findings are presented. On the basis of line drawings of females, an original illustrated key to all known species in the Middle East and North Africa is provided.

\section{Material and methods}

New material was collected during 2011-2013 from various regions of Saudi Arabia. Plant material with aphid colonies (both live and mummified individuals) was collected from the field and transferred to the laboratory. Then, it was cleaned and subdivided into properly prelabeled plastic rearing jars covered with fine mesh for ventilation. The containers were kept in room temperature for a period of 1-2 weeks until emergence of adult parasitoids. As soon as the adult parasitoids emerged, they were immediately captured using an aspirator and transferred into vials containing 70\% ethyl alcohol. Female parasitoids were dissected and slide mounted in Berlese medium (Tomanović et al. 2018). The external morphology of parasitoids was studied using an Olympus ${ }^{\mathrm{TM}}$ SZX9 (Olympus Corporation, Japan) or a Nikon ${ }^{\mathrm{TM}}$ SMZ645 (Nikon Corporation, Japan) stereo microscopes and a Nikon ${ }^{\mathrm{TM}}$ Eclipse E200 (Nikon Corporation, Japan) or a Leica ${ }^{\mathrm{TM}}$ DMLS phase-contrast (Leica Microsystems GmBH, Wetzlar, Germany) microscopes. Line drawings were traced in Adobe Illustrator CS5 on the digital photographs from the slides, captured using a Canon ${ }^{\mathrm{TM}}$ EOS 700D digital camera (Canon Inc., Japan) directly mounted on the micrsoscope. The ratio measurements were based on the slide-mounted specimens using an ocular micrometer or using tpsDig software (Rohlf 2006) on the digital photographs. Specimens from the following depositories were examined and used to prepare the key and illustrations: Department of Plant Protection, University of Zabol, Iran; Institute of Entomology, Biology Centre, Česke Budějovice, Czech Republic; Institute of Zoology, Faculty of Biology, University of Belgrade, Serbia; Laboratory of Agricultural Zoology and Entomology, Department of Crop Science, Agricultural University of Athens, Greece, and Naturalis Biodiversity Centre, Leiden, The Netherlands. The new series of specimens from Saudi Arabia were deposited in the Collection of Department of Plant Protection, University of Zabol (DPPZ). The rest of the data have been extracted from literature that refers to taxonomy, faunal diversity and biology of Aphidiinae parasitoids in various countries of the Middle East and North African countries. The distribution of the listed species is also presented in this study. Terminology of morphological characters 
for the parasitoids follows Gärdenfors (1986) (for the sculpture of propodeum) and Sharkey \& Wharton (1997). Aphid nomenclature follows Remaudière \& Remaudière (1997) and in few cases updated according to Nieto Nafria et al. (2011) and Favret (2019).

\title{
Results
}

\section{Aphid parasitoids - host aphids - distribution and Taxonomy}

A total of 108 Aphidiinae species, belonging to 18 genera, associated with 240 aphid species in 16 countries of the Middle East and North Africa are reviewed. 743 host aphid-parasitoid associations are listed in the target area. Doubtful records are marked with "?", i.e., associations that fall out of parasitoids' known host range. These records need to be verified but are kept in the present account, since they may be worthy as issues to look upon in future studies in the target region and elsewhere.

\author{
Class Insecta L., 1758 \\ Order Hymenoptera L., 1758 \\ Family Braconidae Nees, 1811 \\ Subfamily Aphidiinae Haliday, 1833 \\ Aclitus obscuripennis Förster, 1862 \\ Figs 1, 73, 176, 272, 374, 510
}

\section{Host records}

Lacking host data (Farahani et al. 2017).

\section{Distribution in the Middle East and North Africa}

Iran (Farahani et al. 2017).

\section{General distribution}

Eastern (Iran) and Western Palaearctic (Europe).

Adialytus ambiguus Haliday, 1834

Figs 2, 74, 177, 273, 375, 500, 511

\section{Host records}

Sipha elegans Del Guercio (Elmali 1997; Uysal et al. 2004; Rakhshani et al. 2008b, 2012a; Barahoei et al. 2013; Taheri \& Rakhshani 2013); Sipha flava (Forbes) (Nazari et al. 2012; Rakhshani et al. 2012a); Sipha maydis Passerini (Mescheloff \& Rosen 1990a; Starý et al. 2000; Nazari et al. 2012; Rakhshani et al. 2012a, 2012b; Barahoei et al. 2013); Sipha sp. (Starý 1979).

\section{Distribution in the Middle East and North Africa}

Egypt (El-Ghiet et al. 2014 - as Adialytus cf. ambiguus (Haliday, 1834)); Iran (Starý 1979; Starý et al. 2000; Nazari et al. 2012; Rakhshani et al. 2012a, 2012b; Barahoei et al. 2013; Taheri \& Rakhshani 2013); Israel (Mescheloff \& Rosen 1990a); Turkey (Starý 1976; Central part - Elmali 1997; Western part - Uysal et al. 2004).

\section{General distribution}

Holarctic, Oriental. 
RAKHSHANI E. et al., Aphidiinae of the Middle East and North Africa

Adialytus salicaphis (Fitch, 1855)

Figs 3, 75, 178, 274, 376, 501, 512

\section{Host records}

Chaitophorus euphraticus Hodjat (Starý et al. 2000; Rakhshani et al. 2007b, 2012a; Talebi et al. 2009); Chaitophorus leucomelas Koch (Ölmez \& Ulusoy 2003; Uysal et al. 2004; Rakhshani et al. 2007b, 2012a; Barahoei et al. 2013; Taheri \& Rakhshani 2013); Chaitophorus populeti (Panzer) (Rakhshani et al. 2007b, 2012a); Chaitophorus populialbae (Boyer de Fonscolombe) (Starý et al. 2000; Rakhshani et al. 2007b, 2012a; Barahoei et al. 2013); Chaitophorus remaudierei Pintera (Starý et al. 2000; Rakhshani et al. 2007b, 2012a; Talebi et al. 2009; Nazari et al. 2012); Chaitophorus salijaponicus Essig \& Kuwana (Starý et al. 2000; Uysal et al. 2004; Rakhshani et al. 2007b, 2012; Nazari et al. 2012; Barahoei et al. 2013; Taheri \& Rakhshani 2013); Chaitophorus truncatus (Hausmann) (Rakhshani et al. 2007b; Talebi et al. 2009); Chaitophorus vitellinae (Schrank) (Rakhshani et al. 2007b, 2012a; Talebi et al. 2009; Barahoei et al. 2013); Chaitophorus spp. (Starý \& Kaddou 1971; Starý 1976, 1979; Starý et al. 2000; Barahoei et al. 2012, 2013; Rakhshani et al. 2012a, 2012b).

\section{Distribution in the Middle East and North Africa}

Iran (Starý 1979; Starý et al. 2000; Barahoei et al. 2012; Nazari et al. 2012; Rakhshani et al. 2007b, 2012a, 2012b; Taheri \& Rakhshani 2013); Iraq (Starý \& Kaddou 1971; Starý 1976); Turkey (Eastern part - Ölmez \& Ulusoy 2003; Western part - Uysal et al. 2004; Erdoğan et al. 2008).

\section{General distribution}

Holarctic, Neotropical, Oriental.

Adialytus thelaxis Starý, 1961

Figs 4, 76, 179, 275, 377, 502, 513

\section{Host records}

Thelaxes confertae Börner (Mescheloff \& Rosen 1990a); Thelaxes suberi (Del Guercio) (Starý 1969a, 1969b, 1976; Starý \& Kaddou 1971; Starý 1979; Babaee et al. 2000; Starý et al. 2000; Nazari et al. 2012; Rakhshani et al. 2012a); Thelaxes sp. (Starý 1976).

\section{Distribution in the Middle East and North Africa}

Iran (Starý 1979; Babaee et al. 2000; Starý et al. 2000; Nazari et al. 2012; Rakhshani et al. 2012a); Iraq (Starý 1969a, 1969b, 1976; Starý \& Kaddou 1971); Israel (Mescheloff \& Rosen 1990a); Turkey (Starý 1976).

\section{General distribution}

Eastern (Iran) and Western Palaearctic.

Adialytus veronicaecola (Starý, 1978)

Figs 5, 77, 180, 276, 378, 503, 514

\section{Host records}

Aphis craccivora Koch, Aphis gossypii Glover (Rakhshani et al. 2012a). 


\section{Distribution in the Middle East and North Africa}

Iran (Rakhshani et al. 2012a); Turkey - Western part (Erdoğan \& Akar 2018).

\section{General distribution}

Eastern (Iran, Kazakhstan) and Western Palaearctic (Turkey).

Aphidius absinthii Marshall, 1896

Figs 6, 78, 181, 277, 379, 476, 515

\section{Host records}

Macrosiphoniella abrotani (Walker) (Starý et al. 2000; Rakhshani et al. 2008a, 2011; Talebi et al. 2009); Macrosiphoniella artemisiae (Boyer de Fonscolombe) (Starý 1976; Aslan et al. 2004; Uysal et al. 2004; Rakhshani et al. 2008a, 2011; Talebi et al. 2009); Macrosiphoniella helichrysi Remaudière (Starý et al. 2000; Rakhshani et al. 2011); Macrosiphoniella oblonga (Mordvilko) (Starý et al. 2000; Rakhshani et al. 2008a, 2011; Talebi et al. 2009); Macrosiphoniella pulvera (Walker) (Starý 1979; Starý et al. 2000; Rakhshani et al. 2011); Macrosiphoniella riedeli Szelegiewicz (Mescheloff \& Rosen 1990b; Rakhshani et al. 2011); Macrosiphoniella tuberculata (Nevsky) (Starý 1979; Starý et al. 2000; Rakhshani et al. 2011; Mescheloff \& Rosen 1990b - as Macrosiphoniella nr. macrura Hille Ris Lambers); Macrosiphoniella sp. (Starý et al. 2000; Rakhshani et al. 2011).

\section{Distribution in the Middle East and North Africa}

Iran (Starý 1979; Starý et al. 2000; Talebi et al. 2009; Rakhshani et al. 2008a, 2011); Israel (Mescheloff \& Rosen 1990b); Turkey (Starý 1976; Central part - Aslan et al. 2004; Uysal et al. 2004; Western part Erdoğan et al. 2008).

\section{General distribution}

Holarctic, Oriental.

Aphidius arvensis (Starý, 1960)

Figs 7, 79, 182, 278, 380, 516

\section{Host records}

Coloradoa achilleae Hille Ris Lambers (Starý 1979; Starý et al. 2000; Talebi et al. 2009; Nazari et al. 2012; Barahoei et al. 2013; Taheri \& Rakhshani 2013).

\section{Distribution in the Middle East and North Africa}

Iran (Starý 1979; Starý et al. 2000; Talebi et al. 2009; Nazari et al. 2012; Barahoei et al. 2013; Taheri \& Rakhshani 2013).

\section{General distribution}

Eastern (Iran) and Western Palaearctic (Europe).

Aphidius asteris Haliday, 1834

Figs 80, 183, 279, 381, 477, 517

\section{Host records}

Macrosiphoniella spp. (Rakhshani et al. 2011). 


\section{Distribution in the Middle East and North Africa}

Iran (Rakhshani et al. 2011); Egypt (El-Ghiet et al. 2014).

\section{General distribution}

Holarctic, Oriental.

\section{Remarks}

Record of Aphidius asteris in association with alfalfa fields (El-Ghiet et al. 2014) can be a reason of migration from adjacent plants infested with Macrosiphoniella aphids/or a misidentification.

Aphidius avenae Haliday, 1834

Figs 81, 184, 280, 382, 478, 518

\section{Material examined}

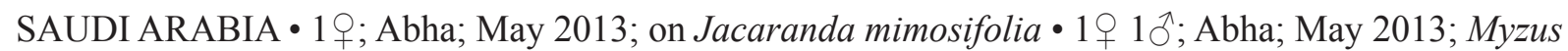
persicae (Sulzer) on Lectuca virosa -1q 1§; Abha; Jun. 2012; Acyrthosiphon pisum (Harris) on Trifolium alexandrinum; Z. Ahmad, leg. (DPPZ).

\section{Host records}

Acyrthosiphon pisum (Harris) (Tremblay et al. 1985; Laamari et al. 2011); Aphis sp. (Uysal et al. 2004); Hyperomyzus lactucae (L.) (Laamari et al. 2011); Myzus persicae (Sulzer) (Uysal et al. 2004); Rhopalosiphum maidis (Fitch) (Taheri \& Rakhshani 2013).

\section{Distribution in the Middle East and North Africa}

Algeria (Laamari et al. 2011); Iran (Taheri \& Rakhshani 2013); Lebanon (Tremblay et al. 1985); Morocco (Bleton \& Fieuzet 1939); Turkey (Central part - Erdoğan et al. 2008; Western part - Uysal et al. 2004; Erdoğan et al. 2008; Akar \& Erdoğan 2017 - as Aphidius picipes (Nees 1811)); Saudi Arabia (new record).

\section{General distribution}

Holarctic, Oriental.

Aphidius banksae Kittel, 2016

Figs 82, 185, 281, 383, 479, 519

\section{Host records}

Acyrthosiphon pisum (Harris) (Chen et al. 1991).

\section{Distribution in the Middle East and North Africa}

Israel, Turkey - Eastern part (Chen et al. 1991).

\section{General distribution}

Western Palaearctic, Nearctic (imported to USA, not recovered again).

\section{Remarks}

All records of Aphidius staryi Chen \& Luhman, 1991 (in Chen et al. 1991) refer to Aphidius banksae Kittel, 2016. 
Aphidius cingulatus Ruthe, 1859

Figs $8,83,186,282,384,480,520$

\section{Host records}

Pterocomma pilosum Buckton (Starý 1979; Starý et al. 2000; Rakhshani et al. 2007b, 2008a, 2012b; Talebi et al. 2009); Pterocomma populeum (Kaltenbach) (Starý 1979; Rakhshani et al. 2007b, 2008a, 2012b); Pterocomma sp. (Starý et al. 2000); Lacking host data (Düzgüneş et al. 1982; Uysal et al. 2004).

\section{Distribution in the Middle East and North Africa}

Iran (Starý 1979; Starý et al. 2000; Rakhshani et al. 2007b, 2008a); Turkey (Central part - Düzgüneş et al. 1982; Uysal et al. 2004).

\section{General distribution}

Holarctic, Neotropical, Oriental.

Aphidius colemani Viereck, 1912

Figs 84, 187, 283, 385, 481, 521

\section{Host records}

Acyrthosiphon gossypii Mordvilko (?), Acyrthosiphon pisum (Harris) (?) (Starý \& Erdelen 1982); Aphis aurantii Boyer de Fonscolombe (Rosen 1967, 1969; Avidov \& Harpaz 1969; Starý 1976; Tremblay et al. 1985; Yumruktepe \& Uygun 1994; Uysal et al. 2004; Yoldaş et al. 2011); Aphis chloris Koch (Mescheloff \& Rosen 1990b); Aphis craccivora Koch (Starý \& Erdelen 1982; Mescheloff \& Rosen 1990b; Ölmez \& Ulusoy 2003; Uysal et al. 2004; Yoldaş et al. 2011; Ahmad \& Bakr 2013; Satar et al. 2014); Aphis epilobiaria Theobald (Mescheloff \& Rosen 1990b); Aphis fabae Scopoli (Abou-Fakhr 1982; Starý \& Erdelen 1982; Tremblay et al. 1985; Mescheloff \& Rosen 1990b - as Aphis citricola van der Goot; Laamari et al. 2012); Aphis gossypii Glover (Mescheloff \& Rosen 1990b; Yoldaş et al. 2002, 2011; Uysal et al. 2004; Boukhris-Bouhachem 2011; Irshaid \& Hasan 2011; Laamari et al. 2012; Satar et al. 2014; Ayadi et al. 2017); Aphis hederae Kaltenbach, Aphis illinoisensis Shimer (El-Gantiry et al. 2012; Havelka et al. 2011); Aphis intybi Koch (Abou-Fakhr 1982; Mescheloff \& Rosen 1990b; AbouFakhr \& Kawar 1998; Laamari et al. 2012); Aphis nerii Boyer de Fonscolombe (Abou-Fakhr 1982; Tremblay et al. 1985; Mescheloff \& Rosen 1990b; Abou-Fakhr \& Kawar 1998; Laamari et al. 2012); Aphis nasturtii Kaltenbach (Starý \& Kaddou 1971; Starý 1976; Mescheloff \& Rosen 1990b - as Aphis zizyphi Theobald); Aphis pomi De Geer (Laamari et al. 2011, 2012); Aphis punicae Passerini (Starý 1976; Al-Azawi 1970; Mescheloff \& Rosen 1990b; Laamari et al. 2012); Aphis solanella Theobald (Ayadi et al. 2017); Aphis umbrella (Börner) (Mescheloff \& Rosen 1990b); Aphis verbasci Schrank (Mescheloff \& Rosen 1990b); Aphis sp. (Starý 1975, 1976); Brachycaudus amygdalinus (Schouteden) (Mescheloff \& Rosen 1990b); Brachycaudus cardui (L.) (Abou-Fakhr 1982; Tremblay et al. 1985; Abou-Fakhr \& Kawar 1998); Brachycaudus helichrysi (Kaltenbach) (Abou-Fakhr 1982; Starý \& Erdelen 1982; Tremblay et al. 1985; Mescheloff \& Rosen 1990b; Abou-Fakhr \& Kawar 1998; Ölmez \& Ulusoy 2003; Uysal et al. 2004; Satar et al. 2014); Capitophorus elaeagni (Del Guercio) (Mescheloff \& Rosen 1990b); Diuraphis noxia (Kurdjumov) (Starý \& Erdelen 1982; Starý et al. 2013); Hayhurstia atriplicis (L.) (Mescheloff \& Rosen 1990b); Hyadaphis foeniculi (Passerini) (Mescheloff \& Rosen 1990b); Hyperomyzus lactucae (L.) (?) (Güz \& Kilinçer 2005); Macrosiphum euphorbiae (Thomas) (Mescheloff \& Rosen 1990b); Macrosiphum rosae (L.) (?) (Mescheloff \& Rosen 1990b); Myzus persicae (Sulzer) (Abou-Fakhr 1982; Starý \& Erdelen 1982; Tremblay et al. 1985; Mescheloff \& Rosen 1990b; Abou-Fakhr \& Kawar 1998; Güz \& Kilinçer 2005; Yoldaş et al. 2011; Laamari et al. 2012; Ahmad \& Bakr 2013; Satar et al. 2014; Hasan 2016); Ovatus mentharius (van der Goot) (Güz \& Kilinçer 2005); Rhopalosiphum maidis (Fitch) (Starý \& Erdelen 1982; Ghanim \& El-Adl 1983; Mescheloff \& Rosen 
1990b; El-Heneidy et al. 2001, 2002, 2004; Sobhy et al. 2004; Güz \& Kilinçer 2005; Gadallah et al. 2017); Rhopalosiphum nymphaeae (L.) (Starý 1976); Rhopalosiphum padi (L.) (Ghanim \& El-Adl 1983; Tremblay et al. 1985; Mescheloff \& Rosen 1990b; Abdel-Rahman et al. 2000; El-Heneidy et al. 2001, 2002, 2004; Sobhy et al. 2004; Abdel-Rahman 2005; Slman 2006; Gadallah et al. 2017); Schizaphis graminum (Rondani) (Starý \& Erdelen 1982; Ghanim \& El-Adl 1983; Mescheloff \& Rosen 1990b; Abdel-Rahman et al. 2000; El-Heneidy et al. 2001, 2002, 2004; Sobhy et al. 2004; Abdel-Rahman 2005; Slman 2006); Schizaphis rosazevedoi Ilharco (Mescheloff \& Rosen 1990b); Sitobion avenae (Fabricius) (Ghanim \& El-Adl 1983; El-Heneidy et al. 2001, 2002; Sobhy et al. 2004; Gadallah et al. 2017); Sitobion fragariae (Walker) (Mescheloff \& Rosen 1990b).

\section{Distribution in the Middle East and North Africa}

Algeria (Starý 1975, 1976; Laamari et al. 2011, 2012); Egypt (Starý 1976; Ghanim \& El-Adl 1983; Abdel-Rahman et al. 2000; El-Heneidy \& Abdel-Samad 2001; El-Heneidy et al. 2001, 2002, 2003, 2004; Sobhy et al. 2004; Abdel-Rahman 2005; Slman 2006; El-Gantiry et al. 2012; El-Ghiet et al. 2014; Gadallah et al. 2017); Iraq (Al-Azawi 1970; Starý \& Kaddou 1971; Starý 1976); Israel (Rosen 1967, 1969; Avidov \& Harpaz 1969; Starý 1970, 1975, 1976; Mescheloff \& Rosen 1990b); Jordan (Irshaid \& Hasan 2011; Hasan 2016); Lebanon (Starý 1976; Abou-Fakhr 1982; Tremblay et al. 1985; Abou-Fakhr \& Kawar 1998); Libya (Havelka et al. 2011; Tomanović et al. 2014); Morocco (Starý \& Sekkat 1987); Palestine (Samara \& Qubbaj 2012); Saudi Arabia (Ahmad \& Bakr 2013); Syria (Starý 1975, 1976); Tunisia (Starý 1976; Ben Halima \& Ben Hamouda 2005; Boukhris-Bouhachem 2011; Ayadi et al. 2017); Turkey (Starý 1976; Uysal et al. 2004; Eastern part - Tozlu et al. 2002; Ölmez \& Ulusoy 2003; Central part - Yumruktepe \& Uygun 1994; Güz \& Kilinçer 2005; Satar et al. 2014; Western part - Yoldaş et al. 2002, 2011; Erdoğan et al. 2008; Akar \& Erdoğan 2017); UAE (Starý et al. 2013); Yemen (Starý \& Erdelen 1982; Starý et al. 2013).

\section{General distribution}

Cosmopolitan.

\section{Remarks}

All evidence from Iran (Starý 1979, Starý et al. 2000; Rakhshani et al. 2008a), refer to Aphidius platensis (Tomanović et al. 2014). Identification-revision of this species group is recommended. Description and illustration of Aphidius colemani in Mescheloff \& Rosen 1990b, clearly represent the diagnostic characters of Aphidius platensis.

Aphidius eadyi Starý, González \& Hall, 1980

Figs $9,85,188,285,386,482,522$

\section{Host records}

Acyrthosiphon pisum (Harris) (González et al. 1978; Starý 1979; Starý et al. 1980, Starý \& Sekkat 1987; Starý et al. 2000; Ölmez \& Ulusoy 2003; Uysal et al. 2004; Rakhshani et al. 2006a, 2008a; Laamari et al. 2012; Nazari et al. 2012; Barahoei et al. 2013; Chaouche \& Laamari 2015).

\section{Distribution in the Middle East and North Africa}

Algeria (Laamari et al. 2012; Chaouche \& Laamari 2015); Iran (Starý 1979; Starý et al. 1980, 2000; Rakhshani et al. 2006a, 2008a; Nazari et al. 2012; Barahoei et al. 2013); Morocco (Starý \& Sekkat 1987; Starý et al. 1980); Turkey (Central and Eastern parts - Ölmez \& Ulusoy 2003; Uysal et al. 2004; Western part - Erdoğan et al. 2008; Akar \& Erdoğan 2017). 


\title{
General distribution
}

Eastern and Western Palaearctic, Nearctic (imported), Oceanic.

Aphidius eglanteriae Haliday, 1834

Figs 86, 189, 284, 387, 523

\section{Host records}

Chaetosiphon tetrarhodum (Walker) (Barjadze et al. 2010).

\section{Distribution in the Middle East and North Africa}

Turkey (Western part - Barjadze et al. 2010).

\section{General distribution}

Western Palaearctic (Europe).

\author{
Aphidius ervi Haliday, 1834
}

Figs 10, 87, 190, 286, 388, 483, 524

\section{Host records}

Acyrthosiphon gossypii Mordvilko (Laamari et al. 2011, 2012; Barahoei et al. 2013); Acyrthosiphon ilka Mordvilko (as Acyrthosiphon bidentis Eastop - Starý 1976); Acyrthosiphon kondoi Shinji (González et al. 1978; Starý et al. 2000; Rakhshani et al. 2006a, 2008a, 2008b; Nazari et al. 2012; Barahoei et al. 2013); Acyrthosiphon lactucae (Passerini) (Zeren \& Düzgüneş 1983; Uysal et al. 2004); Acyrthosiphon pisum (Harris) (Bodenheimer \& Swirski 1957; Eady 1969; Avidov \& Harpaz 1969; Starý 1976; Starý \& González 1978; González et al. 1978; Monajemi \& Esmaili 1981; Tremblay et al. 1985; Talebi et al. 2009; Nazari et al. 2012; Taheri \& Rakhshani 2013; Barahoei et al. 2013; Düzgüneş et al. 1982; Zeren \& Düzgüneş 1983; Rasulian 989; Starý \& Sekkat 1987; Mescheloff \& Rosen 1990b; Starý et al. 2000; Uysal et al. 2004; Rakhshani et al. 2006a, 2008a, 2008b; Laamari et al. 2011, 2012; Rakhshani et al. 2012b; Chaouche \& Laamari 2015); Aphis craccivora Koch (Zeren \& Düzgüneş 1983; Laamari et al. 2011; Chaouche \& Laamari 2015); Aphis fabae Scopoli (Zeren \& Düzgüneş 1983; Uysal et al. 2004; Laamari et al. 2011); Capitophorus elaeagni (Del Guercio); Dysaphis foeniculus (Theobald) (Ayadi et al. 2017); Dysaphis spp., Hyperomyzus lactucae (L.) (Laamari et al. 2011, 2012); Lipaphis pseudobrassicae (Davis) (Chaouche \& Laamari 2015); Macrosiphum euphorbiae (Thomas) (Starý 1976; Abou-Fakhr 1982; Tremblay et al. 1985; Mescheloff \& Rosen 1990b; Zeren \& Düzgüneş 1983; Ben Halima \& Ben Hamouda 1993; Abou-Fakhr \& Kawar 1998; Uysal et al. 2004; Rakhshani et al. 2008a; Laamari et al. 2011, 2012); Metopolophium dirhodum (Walker) (Starý \& Sekkat 1987; Elmali \& Toros 1994; Gülçlü \& Özbek 2002; Uysal et al. 2004); Microlophium carnosum (Buckton) (Rakhshani et al. 2008a; Talebi et al. 2009); Myzus persicae (Sulzer) (Starý 1976; Zeren \& Düzgüneş 1983; Starý \& Sekkat 1987; Ben Halima \& Ben Hamouda 1993; Uysal et al. 2004; Rakhshani et al. 2008a; Laamari et al. 2012; Barahoei et al. 2013; Chaouche \& Laamari 2015); Rhopalosiphum padi (L.) (Laamari et al. 2011; Taheri \& Rakhshani 2013; Gadallah et al. 2017); Schizaphis graminum (Rondani) (Starý \& Sekkat 1987); Sitobion avenae (Fabricius) (Elmali \& Toros 1994; Darvish-Mojeni \& Bayat-Asadi 1995; Uysal et al. 2004; Rakhshani et al. 2008a; Laamari et al. 2011, 2012; Barahoei et al. 2013; Ayadi et al. 2017; Gadallah et al. 2017); Sitobion fragariae (Walker) (Laamari et al. 2011, 2012); Uroleucon aeneum (Hille Ris Lambers) (?); Uroleucon sonchi (L.) (?) (Laamari et al. 2011); Wahlgreniella nervata (Gillette) (Barahoei et al. 2013). 


\section{Distribution in the Middle East and North Africa}

Algeria (Starý 1976; Laamari et al. 2011, 2012; Chaouche \& Laamari 2015); Egypt (Gadallah et al. 2017); Iran (Starý 1979; Starý et al. 2000; Rakhshani et al. 2006a, 2008a, 2008b, 2012b; Nazari et al. 2012; Barahoei et al. 2013; Taheri \& Rakhshani 2013); Iraq (Al-Azawi 1970; Starý 1976); Israel (Bodenheimer \& Swirski 1957; Avidov \& Harpaz 1969; Starý 1976; Mescheloff \& Rosen 1990b); Lebanon (Mackauer \& Starý 1967; Starý 1976; Marsh 1977; Abou-Fakhr 1982; Tremblay et al. 1985; Abou-Fakhr \& Kawar 1998); Morocco (Mimeur 1934; Eady 1969; Starý 1976; Starý \& Sekkat 1987; González et al. 1978); Palestine (Samara \& Qubbaj 2012); Tunisia (Ben Halima \& Ben Hamouda 1993; Ayadi et al. 2017); Turkey (Eastern part - Elmali \& Toros 1994; Gülçlü \& Özbek 2002; Central part Zeren \& Düzgüneş 1983; Uysal et al. 2004; Erdoğan et al. 2008; Western part - Akar \& Erdoğan 2017); UAE (Starý et al. 2013); Yemen (Starý et al. 2013).

\section{General distribution}

Cosmopolitan.

Aphidius funebris Mackauer, 1961

Figs 11, 88, 191, 287, 389, 484, 525

\section{Host records}

Aphis fabae Scopoli (?) (Chaouche \& Laamari 2015); Brachycaudus cardui (L.) (?) (Güz \& Kilinçer 2005; Laamari et al. 2011, 2012); Hyperomyzus lactucae (L.) (?) (Laamari et al. 2011); Uroleucon acroptilidis Kadyrbekov, Renxin \& Shao (Barahoei et al. 2013); Uroleucon aeneum (Hille Ris Lambers) (Güz \& Kilinçer 2005; Laamari et al. 2011, 2012); Uroleucon chondrillae (Nevsky) (Starý 1979; Starý et al. 2000; Rakhshani et al. 2008a); Uroleucon cichorii (Koch) (Rakhshani et al. 2008a); Uroleucon compositae (Theobald) (Laamari et al. 2012; Rakhshani et al. 2008a; Talebi et al. 2009; Nazari et al. 2012; Barahoei et al. 2013); Uroleucon erigeronense (Thomas) (Rakhshani et al. 2008a; Talebi et al. 2009); Uroleucon jaceae (L.) (Al-Azawi 1970; Starý \& Kaddou 1971; Starý 1976; Güz \& Kilinçer 2005; Rakhshani et al. 2008a; Talebi et al. 2009; Laamari et al. 2011, 2012; Nazari et al. 2012; Barahoei et al. 2013; Taheri \& Rakhshani 2013); Uroleucon sonchi (L.) (Al-Azawi 1970; Starý \& Kaddou 1971; Starý 1976; Düzgüneş et al. 1982; Starý \& Sekkat 1987; Uysal et al. 2004; Güz \& Kilinçer 2005; Rakhshani et al. 2008a; Laamari et al. 2011, 2012; Barahoei et al. 2013; Taheri \& Rakhshani 2013; Chaouche \& Laamari 2015; Ayadi et al. 2017); Uroleucon sp. (Starý 1976; Aslan et al. 2004; Uysal et al. 2004; Barahoei et al. 2012, 2013).

\section{Distribution in the Middle East and North Africa}

Algeria (Starý 1976; Laamari et al. 2011, 2012; Chaouche \& Laamari 2015); Iran (Starý 1979; Starý et al. 2000; Rakhshani et al. 2008a; Barahoei et al. 2012, 2013; Nazari et al. 2012; Taheri \& Rakhshani 2013); Iraq (Al-Azawi 1970; Starý \& Kaddou 1971; Starý 1976); Morocco (Starý \& Sekkat 1987); Turkey (Central part - Düzgüneş et al. 1982; Aslan et al. 2004; Güz \& Kilinçer 2005; Eastern part Uysal et al. 2004; Western part - Erdoğan et al. 2008; Akar \& Erdoğan 2017).

\section{General distribution}

Eastern and Western Palaearctic, Oceanic, Oriental.

Aphidius hieraciorum Starý, 1962

Figs 89, 192, 288, 390, 526

\section{Host records}

Nasonovia ribisnigri (Mosley) (Nazari et al. 2012); Nasonovia sp. (Mossadegh et al. 2011). 


\section{Distribution in the Middle East and North Africa}

Iran (Mossadegh et al. 2011; Nazari et al. 2012).

\section{General distribution}

Eastern and Western Palaearctic.

Aphidius iranicus Rakhshani \& Starý, 2007

Figs 90, 193, 289, 391, 527

\section{Host records}

Titanosiphon neoartemisiae (Takahashi) (Tomanović et al. 2007; Talebi et al. 2009 - as Titanosiphon bellicosum Nevesky).

\section{Distribution in the Middle East and North Africa}

Iran (Tomanović et al. 2007; Talebi et al. 2009).

\section{General distribution}

This species has not been recorded outside Iran.

Aphidius matricariae Haliday, 1834

Figs 12, 91, 194, 290, 392, 485, 528

\section{Material examined}

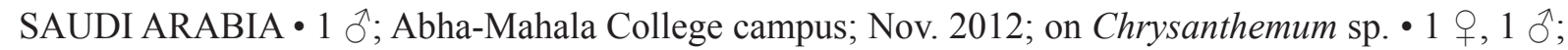

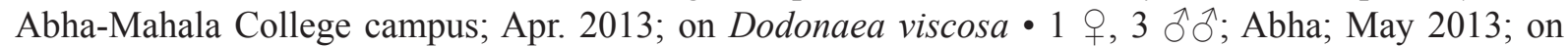

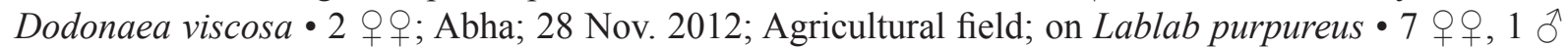
Abha; Nov. 2012; on Lablab purpureus • 1 +, 6 ठ $\sigma^{\lambda}$; Abha-Mahala College campus; Nov. 2012; on

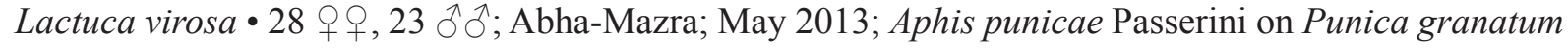

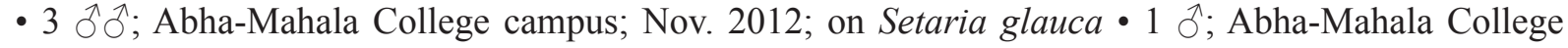

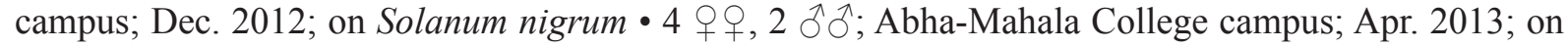
Solanum nigrum • 1 §’; Abha-Mahala College campus; Nov. 2012; on Sonchus oleraceus; Z. Ahmed leg. (DPPZ).

\section{Host records}

Acyrthosiphon gossypii Mordvilko (?) (Chaouche \& Laamari 2015); Amegosiphon platicaudum (Narzikulov) (Nazari et al. 2012); Aphis affinis Del Guercio (Starý \& Kaddou 1971; Starý 1976; Aslan et al. 2004; Uysal et al. 2004; Rakhshani et al. 2012b; Nazari et al. 2012; Barahoei et al. 2013); Aphis aurantii Boyer de Fonscolombe (Hussein \& Kawar 1984; Tremblay et al. 1985; Ben Halima et al. 1994; Uysal et al. 2004; Ben Halima \& Ben Hamouda 2005; Boukhris-Bouhachem 2011); Aphis craccivora Koch (Starý \& Kaddou 1971; Starý 1976; Mescheloff \& Rosen 1990b; Rakhshani et al. 2008a; Talebi et al. 2009; Abdel-Samad \& Ahmed 2009; Mossadegh et al. 2011; Laamari et al. 2011, 2012; Nazari et al. 2012; Barahoei et al. 2013; Taheri \& Rakhshani 2013; Chaouche \& Laamari 2015); Aphis crepidis (Börner) (Starý et al. 2000); Aphis dlabolai Holman (Barahoei et al. 2013); Aphis euphorbiae Kaltenbach (Rakhshani et al. 2008a; Talebi et al. 2009; Mossadegh et al. 2011; Barahoei et al. 2013); Aphis fabae Scopoli (Abou-Fakhr 1982; Tremblay et al. 1985; Mescheloff \& Rosen 1990b; Ben Halima \& Ben Hamouda 1993; Abou-Fakhr \& Kawar 1998; Rakhshani et al. 2008a; Talebi et al. 2009; Mossadegh et al. 2011; Laamari et al. 2011, 2012; Nazari et al. 2012; Barahoei et al. 2013; Taheri \& Rakhshani 2013; Ayadi et al. 2017); Aphis gossypii Glover (Starý 1979; Abou-Fakhr 1982; Tremblay et al. 1985; Mescheloff \& Rosen 1990b; Ben 
Halima \& Ben Hamouda 1993; Ben Halima et al. 1994; Starý et al. 2000; Ben Halima \& Ben Hamouda 2005; Rakhshani et al. 2008a; Talebi et al. 2009; Boukhris-Bouhachem 2011; Mossadegh et al. 2011; Laamari et al. 2011, 2012; Nazari et al. 2012; Ahmad \& Bakr 2013; Barahoei et al. 2013; Satar et al. 2014; Ayadi et al. 2017); Aphis hederae Kaltenbach (Mescheloff \& Rosen 1990b); Aphis illinoisensis Shimer (Barjadze et al. 2010; Havelka et al. 2011); Aphis intybi Koch (Barahoei et al. 2013; Taheri \& Rakhshani 2013); Aphis nasturtii Kaltenbach (Starý 1976; Mescheloff \& Rosen 1990b - as Aphis zizyphi Theobald; Nazari et al. 2012); Aphis nerii Boyer de Fonscolombe (AbouFakhr 1982; Tremblay et al. 1985; Ben Halima et al. 1994; Abou-Fakhr \& Kawar 1998; Ben Halima \& Ben Hamouda 2005; Laamari et al. 2012); Aphis parietariae Theobald (Starý \& Kaddou 1971; Starý 1976); Aphis plantaginis Goeze (Barahoei et al. 2013); Aphis pomi De Geer (Ölmez \& Ulusoy 2003; Rakhshani et al. 2008a; Rakhshani 2012; Barahoei et al. 2013); Aphis punicae Passerini (Laamari et al. 2012; Nazari et al. 2012; Barahoei et al. 2013); Aphis solanella Theobald (Barahoei et al. 2013); Aphis spiraecola Patch (Laamari et al. 2012; Rakhshani 2012); Aphis umbrella (Börner) (Schlinger \& Mackauer 1963; Abou-Fakhr 1982; Tremblay et al. 1985; Mescheloff \& Rosen 1990b; Abou-Fakhr \& Kawar 1998; Starý et al. 2000; Rakhshani et al. 2008a; Talebi et al. 2009; Laamari et al. 2011, 2012; Nazari et al. 2012); Aulacorthum solani (Kaltenbach) (Hussein \& Kawar 1984); Brachycaudus amygdalinus (Schouteden) (Mescheloff \& Rosen 1990b; Laamari et al. 2011, 2012; Nazari et al. 2012; Rakhshani 2012); Brachycaudus cardui (L.) (Ölmez \& Ulusoy 2003; Rakhshani et al. 2008a; Laamari et al. 2011, 2012; Rakhshani 2012); Brachycaudus divaricatae Shaposhnikov (Jafari et al. 2011; Jafari \& Modarres Awal 2012); Brachycaudus helichrysi (Kaltenbach) (Starý 1976; Erkin 1983; Mescheloff \& Rosen 1990b; Mokhtari et al. 2000; Starý et al. 2000; Uysal et al. 2004; Rakhshani et al. 2008a, 2012b; Talebi et al. 2009; Mossadegh et al. 2011; Rakhshani 2012; Jafari et al. 2011; Laamari et al. 2011, 2012; Jafari \& Modarres Awal 2012; Nazari et al. 2012; Barahoei et al. 2013; Satar et al. 2014; Chaouche \& Laamari 2015); Brachycaudus persicae (Passerini) (Rakhshani 2012); Brachycaudus tragopogonis (Kaltenbach) (Aslan et al. 2004; Uysal et al. 2004; Rakhshani et al. 2008a; Talebi et al. 2009); Brevicoryne brassicae (L.) (?) (Chaouche \& Laamari 2015); Capitophorus elaeagni (Del Guercio) (Mescheloff \& Rosen 1990b; Laamari et al. 2011, 2012; Chaouche \& Laamari 2015); Capitophorus inulae (Passerini) (Mescheloff \& Rosen 1990b); Diuraphis noxia (Kurdjumov) (González et al. 1992; Zareh et al. 1995; Starý et al. 2000; Uysal et al. 2004; Laamari et al. 2011, 2012, 2016); Dysaphis crataegi (Kaltenbach) (Abou-Fakhr 1982; Tremblay et al. 1985; Abou-Fakhr \& Kawar 1998); Dysaphis devecta (Walker) (Starý et al. 2000; Aslan \& Karaca 2005); Dysaphis foeniculus (Theobald) (Chaouche \& Laamari 2015); Dysaphis lappae (Koch) (Laamari et al. 2011, 2012); Dysaphis plantaginea (Passerini) (Radjabi 1989; Mescheloff \& Rosen 1990b - as Myzus mali Ferrari; Starý et al. 2000; Ölmez \& Ulusoy 2003; Aslan \& Karaca 2005; Laamari et al. 2011, 2012; Mossadegh et al. 2011; Rakhshani 2012); Dysaphis pyri (Boyer de Fonscolombe) (Erkin 1983; Radjabi 1989; Starý et al. 2000; Ölmez \& Ulusoy 2003; Uysal et al. 2004; Laamari et al. 2012); Eucarazzia elegans (Ferrari) (Rakhshani et al. 2008a; Talebi et al. 2009; Barahoei et al. 2013); Hyalopterus amygdali (Blanchard) (?) (Jafari et al. 2011; Jafari \& Modarres Awal 2012; Nazari et al. 2012); Hyalopterus pruni (Geoffroy) (?) (Erkin 1983; Mokhtari et al. 2000; Starý et al. 2000; Uysal et al. 2004; Laamari et al. 2011, 2012); Hyperomyzus lactucae (L.) (?) (Laamari et al. 2011; Mossadegh et al. 2011; Chaouche \& Laamari 2015); Lipaphis erysimi (Kaltenbach) (Mossadegh et al. 2011); Lipaphis lepidii (Nevsky) (Starý 1979; Starý et al. 2000; Mossadegh et al. 2011); Lipaphis pseudobrassicae (Davis) (Barahoei et al. 2013; Chaouche \& Laamari 2015); Macrosiphum euphorbiae (Thomas) (Yoldaş et al. 1990; Mescheloff \& Rosen 1990b; Ben Halima \& Ben Hamouda 1993; Uysal et al. 2004); Macrosiphum rosae (L.) (Ben Halima \& Ben Hamouda 1993; Laamari et al. 2011); Metopolophium dirhodum (Walker) (Starý et al. 2000; Uysal et al. 2004; Rakhshani et al. 2008a, 2008b; Nazari et al. 2012; Barahoei et al. 2013; Taheri \& Rakhshani 2013); Myzus ascalonicus Doncaster (Barahoei et al. 2013); Myzus beybienkoi (Narzykulov) (Starý et al. 2000); Myzus cerasi (Fabricius) (Erkin 1983; Mokhtari et al. 2000; Starý et al. 2000; Uysal et al. 2004; Rakhshani et al. 2008a; Rakhshani 2012); Myzus certus (Walker) 
(Barahoei et al. 2013); Myzus ornatus Laing (Starý et al. 2000); Myzus persicae (Sulzer) (Schlinger \& Mackauer 1963; Rosen 1967, 1969; Avidov \& Kotter 1966; Avidov \& Harpaz 1969; Starý \& Kaddou 1971; Starý 1976, 1979; Abou-Fakhr 1982; Erkin 1983; Tremblay et al. 1985; Karaat \& Goven 1986; Radjabi 1989; Mescheloff \& Rosen 1990b; Ben Halima \& Ben Hamouda 1993; Ben Halima et al. 1994; Abou-Fakhr \& Kawar 1998; Starý et al. 2000; Ölmez \& Ulusoy 2003; Uysal et al. 2004; Ben Halima \& Ben Hamouda 2005; Rakhshani et al. 2008a; Talebi et al. 2009; Boukhris-Bouhachem 2011; Laamari et al. 2011, 2012; Mossadegh et al. 2011; Jafari et al. 2011; Jafari \& Modarres Awal 2012; Barahoei et al. 2012, 2013; Nazari et al. 2012; Rakhshani 2012; Taheri \& Rakhshani 2013; Chaouche \& Laamari 2015; Ayadi et al. 2017); Nasonovia ribisnigri (Mosley) (?) (Laamari et al. 2011, 2012); Ovatus insitus (Walker) (Rakhshani et al. 2008a; Rakhshani 2012); Phorodon humuli (Schrank) (Schlinger \& Mackauer 1963; Starý et al. 2000); Rhopalomyzus sp. (Starý 1979); Rhopalosiphum maidis (Fitch) (Ibrahim 1990a, 1990b; Mescheloff \& Rosen 1990b; Ibrahim \& Afifi 1991; El-Heneidy 1994; Starý et al. 2000; El-Heneidy \& Abdel-Samad 2001; El-Heneidy et al. 2001, 2002, 2003; Abdel-Rahman 2005; Slman 2006; Laamari et al. 2011, 2012; Barahoei et al. 2012, 2013; Nazari et al. 2012; Chaouche \& Laamari 2015; Ayadi et al. 2017); Rhopalosiphum nymphaeae (L.) (Mokhtari et al. 2000; Starý et al. 2000); Rhopalosiphum padi (L.) (Abou-Fakhr 1982; Hussein \& Kawar 1984; Tremblay et al. 1985; Ibrahim 1990b; Mescheloff \& Rosen 1990b; El-Heneidy 1994, Abou-Fakhr \& Kawar 1998; Starý et al. 2000; El-Heneidy et al. 2001, 2002, 2003; Abdel-Rahman 2005; Slman 2006; Rakhshani et al. 2008a, 2008b; Laamari et al. 2011, 2012; Mossadegh et al. 2011; Nazari et al. 2012; Rakhshani 2012; Barahoei et al. 2013; Taheri \& Rakhshani 2013; Chaouche \& Laamari 2015; Gadallah et al. 2017); Schizaphis graminum (Rondani) (Mescheloff \& Rosen 1990b; El-Heneidy 1994; Starý et al. 2000; El-Heneidy \& Abdel-Samad 2001; El-Heneidy et al. 2001, 2002, 2003; Abdel-Rahman 2005; Slman 2006; Rakhshani et al. 2008a, 2008b; Nazari et al. 2012; Barahoei et al. 2013); Schizaphis rosazevedoi Ilharco (Mescheloff \& Rosen 1990b); Sitobion avenae (Fabricius) (Mescheloff \& Rosen 1990b; El-Heneidy 1994; Starý et al. 2000; El-Heneidy et al. 2001, 2002, 2003; Slman 2006; Rakhshani et al. 2008a, 2008b; Laamari et al. 2011, 2012; Mossadegh et al. 2011; Barahoei et al. 2013; Gadallah et al. 2017); Sitobion fragariae (Walker) (Mescheloff \& Rosen 1990b; Barahoei et al. 2013); Uroleucon compositae (Theobald) (?) (Laamari et al. 2011); Uroleucon sonchi (L.) (?) (Mescheloff \& Rosen 1990b); Wahlgreniella nervata (Gillette) (Barahoei et al. 2013).

\section{Distribution in the Middle East and North Africa}

Algeria (Starý 1976; Laamari et al. 2011, 2012; Chaouche \& Laamari 2015); Egypt (Ibrahim 1990a, 1990b; El-Heneidy 1994; El-Heneidy \& Abdel-Samad 2001; El-Heneidy et al. 2001, 2002, 2003, 2004; Abdel-Rahman 2005; Abdel-Samad \& Ahmed 2009; El-Ghiet et al. 2014; Gadallah et al. 2017); Iran (Starý 1979; Starý et al. 2000; Rakhshani et al. 2008a, 2008b; Rakhshani et al. 2012b; Nazari et al. 2012; Rakhshani 2012; Barahoei et al. 2012, 2013; Taheri \& Rakhshani 2013); Iraq (Starý \& Kaddou 1971; Starý 1976); Israel (Schlinger \& Mackauer 1963; Rosen 1967, 1969; Avidov \& Kotter 1966; Avidov \& Harpaz 1969; Starý 1976; Mescheloff \& Rosen 1990b); Lebanon (Abou-Fakhr 1982; Hussein \& Kawar 1984; Tremblay et al. 1985; Abou-Fakhr \& Kawar 1998); Saudi Arabia (Ahmad \& Bakr 2013); Tunisia (Ben Halima \& Ben Hamouda 1993; Ben Halima et al. 1994; Ben Halima \& Ben Hamouda 2005; Boukhris-Bouhachem 2011; Ayadi et al. 2017); Turkey (Starý 1976; Uysal et al. 2004; Eastern part - Karaat \& Goven 1986; Ölmez \& Ulusoy 2003; Central part - Aslan et al. 2004; Satar et al. 2014; Western part - Erkin 1983; Aslan \& Karaca 2005; Erdoğan et al. 2008; Barjadze et al. 2010; Havelka et al. 2011; Akar \& Erdoğan 2017); UAE (Starý et al. 2013); Yemen (Starý et al. 2013).

\section{General distribution}

Cosmopolitan. 
Aphidius microlophii Pennachio \& Tremblay, 1987

Figs 92, 195, 291, 393, 486, 529

\section{Host records}

Lacking host data (Akar \& Erdoğan 2017).

Distribution in the Middle East and North Africa

Turkey (Western part - Akar \& Erdoğan 2017).

\section{General distribution}

Western Palaearctic (Europe).

Aphidius myzocallidis Mescheloff \& Rosen, 1990

Figs 93, 196, 292, 530

\section{Host records}

Myzocallis glandulosa Hille Ris Lambers (Mescheloff \& Rosen 1990b).

Distribution in the Middle East and North Africa

Israel (Mescheloff \& Rosen 1990b).

\section{General distribution}

This species has not been recorded outside Israel.

Aphidius persicus Rakhshani \& Starý, 2006

Figs 13, 94, 197, 293, 394, 487, 531

\section{Host records}

Macrosiphoniella sp., Uroleucon bielawskii (Szelegiewicz), Uroleucon carthami (Hille Ris Lambers) (Barahoei et al. 2013); Uroleucon chondrillae (Nevsky); (Rakhshani et al. 2006b, 2008a, 2011; Nazari et al. 2012; Taheri \& Rakhshani 2013; Barahoei et al. 2013); Uroleucon compositae (Theobald); Uroleucon ochropus (Hille Ris Lambers), (Barahoei et al. 2013); Uroleucon sonchi (L.) (Rakhshani et al. 2008a; Mossadegh et al. 2011; Barahoei et al. 2012, 2013); Uroleucon spp. (Rakhshani et al. 2012b; Barahoei et al. 2012, 2013).

\section{Distribution in the Middle East and North Africa}

Iran (Rakhshani et al. 2006b, 2008a, 2012b; Mossadegh et al. 2011; Barahoei et al. 2012, 2013; Nazari et al. 2012, Taheri \& Rakhshani 2013); Iraq (Rakhshani et al. 2006b).

\section{General distribution}

Western Asia.

Aphidius platensis Brèthes, 1913

Figs 14, 95, 198, 294, 395, 488, 532

\section{Material examined}

SAUDI ARABIA • 1 ㅇ, 1 §ै; Abha-KKU campus; May 2012; Aphis gossypii Glover on Aster squamatus






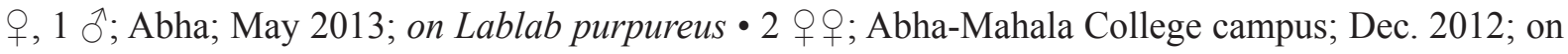
Setaria glauca 1 क; Abha-Mahala College campus; Apr. 2013; Aphis solanella Theobald on Solanum

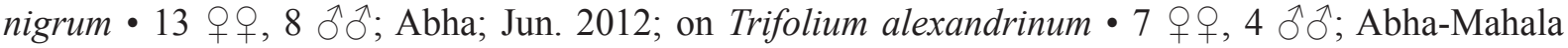
College campus; 6 Mar. 2013; on Trigonella sp.; Z. Ahmad leg. (DPPZ).

\section{Host records}

Amegosiphon platicaudum (Narzikulov) (Nazari et al. 2012); Aphis acetosae L. and Aphis affinis del Guercio (Barahoei et al. 2013); Aphis craccivora Koch (Rakhshani et al. 2005a, 2006a, 2008a; Talebi et al. 2009; Barahoei et al. 2013; Taheri \& Rakhshani 2013); Aphis fabae Scopoli (Rakhshani et al. 2008a, 2012b; Talebi et al. 2009; Barahoei et al. 2013; Taheri \& Rakhshani 2013); Aphis gossypii Glover (Rakhshani et al. 2008a; Talebi et al. 2009; Nazari et al. 2012; Current study), Aphis intybi Koch (Rakhshani et al. 2008a; Talebi et al. 2009; Barahoei et al. 2013; Taheri \& Rakhshani 2013); Aphis nerii Boyer de Fonscolombe (Rakhshani et al. 2008a; Talebi et al. 2009; Barahoei et al. 2013; Taheri \& Rakhshani 2013); Aphis plantaginis Goeze (Barahoei et al. 2013); Aphis punicae Passerini (Rakhshani et al. 2008a, 2012b; Barahoei et al. 2013; Taheri \& Rakhshani 2013); Aphis solanella Theobald (Barahoei et al. 2013; Current study); Aphis rumicis L. (Rakhshani et al. 2012b); Aphis umbrella (Börner) (Rakhshani et al. 2008a; Talebi et al. 2009; Nazari et al. 2012); Brachycaudus amygdalinus (Schouteden) (Rakhshani 2012; Barahoei et al. 2013); Brachycaudus cardui (L.) (Rakhshani et al. 2008a; Talebi et al. 2009; Barahoei et al. 2013; Taheri \& Rakhshani 2013); Brachycaudus helichrysi (Kaltenbach) (Rakhshani et al. 2008a; Talebi et al. 2009; Barahoei et al. 2013); Brachycaudus tragopogonis (Kaltenbach) (Barahoei et al. 2013); Capitophorus elaeagni (Del Guercio) (Rakhshani et al. 2008a; Talebi et al. 2009); Diuraphis noxia (Kurdjumov) (Starý et al. 2000); Dysaphis pulverina ssp. iranica Stroyan, Dysaphis radicola (Mordvilko) (Barahoei et al. 2013); Hayhurstia atriplicis (L.) (Rakhshani et al. 2012b); Hyalopterus amygdali (Blanchard) (Taheri \& Rakhshani 2013); Macrosiphum euphorbiae (Thomas) (Barahoei et al. 2013; Taheri \& Rakhshani 2013); Macrosiphum rosae (L.) (?) (Taheri \& Rakhshani 2013); Metopolophium dirhodum (Walker) (Barahoei et al. 2013); Myzus persicae (Sulzer) (Rakhshani et al. 2008a; Talebi et al. 2009; Mossadegh et al. 2011; Rakhshani 2012; Barahoei et al. 2013; Taheri \& Rakhshani 2013); Phorodon humuli (Schrank) (Rakhshani et al. 2008a; Rakhshani 2012); Rhopalosiphum maidis (Fitch) (Barahoei et al. 2013); Rhopalosiphum padi (L.) (Rakhshani et al. 2008a, 2008b; Nazari et al. 2012; Barahoei et al. 2013); Schizaphis graminum (Rondani) (Starý et al. 2000; Rakhshani et al. 2008a, 2008b; Barahoei et al. 2013); Sitobion avenae (F.) (Starý et al. 2000; Rakhshani et al. 2008a, 2008b; Barahoei et al. 2013).

\section{Distribution in the Middle East and North Africa}

Iran (Starý et al. 2000; Talebi et al. 2009; Mossadegh et al. 2011; Nazari et al. 2012; Rakhshani et al. 2005a, 2006a, 2008a; 2008b, 2012b; Rakhshani 2012; Barahoei et al. 2013; Taheri \& Rakhshani 2013); Saudi Arabia (new record).

\section{General distribution}

Eastern Palaearctic (Middle East), Neotropical (Chile), Oriental.

\section{Remarks}

Based on molecular and morphological studies, this species was separated from Aphidius colemani (Tomanović et al. 2014). All host records of Aphidius colemani Viereck, 1912 in Iran (Barahoei et al. 2014; Farahani et al. 2016) referring to Aphidius platensis. Other records of Aphidius colemani from the Western Asia and records of Aphidius transcaspicus Telenga from the aphids other than Hyalopterus spp. most probably refer to Aphidius platensis. 
Aphidius popovi Starý, 1978

Figs 15, 96, 199, 295, 396, 489, 533

\section{Host records}

Amphorophora catharinae (Nevsky) (Rakhshani et al. 2008a; Talebi et al. 2009; Nazari et al. 2012; Rakhshani et al. 2012b; Barahoei et al. 2013); Metopolophium dirhodum (Walker) (Barahoei et al. 2012, 2013).

\section{Distribution in the Middle East and North Africa}

Iran (Rakhshani et al. 2008a; Talebi et al. 2009; Barahoei et al. 2012; Nazari et al. 2012; Rakhshani et al. 2012b; Barahoei et al. 2013).

\section{General distribution}

Eastern Palaearctic (Central and Western Asia).

Aphidius rhopalosiphi De Stefani-Perez, 1902

Figs 16, 97, 200, 296, 397, 490, 534

\section{Host records}

Diuraphis noxia (Kurdjumov) (Starý et al. 2000; Uysal et al. 2004; Laamari et al. 2011, 2012, 2016); Metopolophium dirhodum (Walker) (Rakhshani et al. 2008a, 2008b; Nazari et al. 2012; Barahoei et al. 2013); Rhopalosiphum maidis (Fitch) (Ghanim \& El-Adl 1983; El-Serafy 1999; Laamari et al. 2011, 2012; Nazari et al. 2012; Gadallah et al. 2017); Rhopalosiphum padi (L.) (Ghanim \& El-Adl 1983; El-Serafy 1999; Starý et al. 2000; Uysal et al. 2004; Rakhshani et al. 2008a, 2008b; Laamari et al. 2011; Nazari et al. 2012; Barahoei et al. 2013; Taheri \& Rakhshani 2013; Chaouche \& Laamari 2015), Schizaphis graminum (Rondani) (Ghanim \& El-Adl 1983; El-Serafy 1999; Rakhshani et al. 2008a, 2008b; Nazari et al. 2012; Barahoei et al. 2013); Sitobion avenae (Fabricius) (Ghanim \& El-Adl 1983; Darvish-Mojeni 1994; El-Serafy 1999; Starý et al. 2000; Rakhshani et al. 2008a, 2008b; Mossadegh et al. 2011; Nazari et al. 2012; Barahoei et al. 2013; Gadallah et al. 2017)

\section{Distribution in the Middle East and North Africa}

Algeria (Laamari et al. 2011, 2012; Chaouche \& Laamari 2015); Egypt (Ghanim \& El-Adl 1983; ElSerafy 1999; Gadallah et al. 2017); Iran (Darvish-Mojeni 1994; Starý et al. 2000; Rakhshani et al. 2008a, 2008b; Mossadegh et al. 2011; Nazari et al. 2012; Barahoei et al. 2013; Taheri \& Rakhshani 2013); Israel (Starý 1981b); Turkey (Central part - Uysal et al. 2004).

\section{General distribution}

Holarctic, Neotropical, Oceanic, Oriental.

Aphidius ribis Haliday, 1834

Figs 98, 201, 297, 398, 535

\section{Host records}

Cryptomyzus ribis (L.) (Düzgüneş et al. 1982; Alaoğlu 1994; Uysal et al. 2004); Myzus persicae (Sulzer)

(?) (Düzgüneş et al. 1982; Uysal et al. 2004).

\section{Distribution in the Middle East and North Africa}

Turkey (Central part - Düzgüneş et al. 1982; Uysal et al. 2004; Eastern part - Alaoğlu 1994). 


\section{General distribution}

Holarctic, Oriental.

Aphidius rosae Haliday, 1833

Figs 17, 99, 202, 298, 399, 491, 536

\section{Host records}

Macrosiphum rosae (L.) (Starý \& Kaddou 1971; Starý 1976; Ben Halima \& Ben Hamouda 1993; Uysal et al. 2004; Mehrparvar et al. 2005; Rakhshani et al. 2008a; Talebi et al. 2009; Mossadegh et al. 2011; Nazari et al. 2012; Barahoei et al. 2013); Sitobion fragariae (Walker) (Düzgüneş et al. 1982; Uysal et al. 2004); Uroleucon sp. (?) (Mescheloff \& Rosen 1990b).

\section{Distribution in the Middle East and North Africa}

Iran (Mehrparvar et al. 2005; Rakhshani et al. 2008a; Mossadegh et al. 2011; Nazari et al. 2012; Barahoei et al. 2013); Iraq (Starý \& Kaddou 1971; Starý 1976); Israel (Mescheloff \& Rosen 1990b); Tunisia (Ben Halima \& Ben Hamouda 1993); Turkey (Central part - Düzgüneş et al. 1982; Uysal et al. 2004; Western part - Akar \& Erdoğan 2017).

\section{General distribution}

Holarctic, Neotropical, Oceanic, Oriental.

Aphidius salicis Haliday, 1834

Figs 18, 100, 203, 299, 400, 492, 537

\section{Host records}

Cavariella aegopodii (Scopoli) (Starý 1979; Düzgüneş et al. 1982; Mescheloff \& Rosen 1990b; Starý et al. 2000; Uysal et al. 2004; Rakhshani et al. 2007b, 2008a; Talebi et al. 2009; Barahoei et al. 2013; Taheri \& Rakhshani 2013); Cavariella aquatica (Gillette \& Bragg) (Starý 1979; Starý et al. 2000); Cavariella aspidaphoides Hille Ris Lambers (Starý 1979; Starý et al. 2000); Cavariella theobaldi (Gillette \& Bragg) (Starý 1979; Düzgüneş et al. 1982; Starý et al. 2000; Uysal et al. 2004); Hyadaphis foeniculi (Passerini) (Talebi et al. 2009).

\section{Distribution in the Middle East and North Africa}

Iran (Starý et al. 2000; Rakhshani et al. 2007b, 2008a; Talebi et al. 2009); Israel (Mescheloff \& Rosen 1990b); Turkey (Central part - Düzgüneş et al. 1982; Uysal et al. 2004).

\section{General distribution}

Australasian, Holarctic, Neotropical, Oceanic, Oriental.

Aphidius setiger (Mackauer, 1961)

Figs 19, 101, 204, 300, 401, 493, 538

\section{Host records}

Periphyllus testudinaceus (Fernie) (Rakhshani et al. 2008a); Periphyllus sp. (Starý 1979; Starý et al. 2000; Nazari et al. 2012).

\section{Distribution in the Middle East and North Africa}

Iran (Starý 1979; Starý et al. 2000; Rakhshani et al. 2008a; Nazari et al. 2012). 


\section{General distribution}

Afrotropical, Holarctic, Oriental.

Aphidius smithi Sharma \& Subba Rao, 1959

Figs 20, 102, 205, 301, 402, 494, 539

\section{Host records}

Acyrthosiphon kondoi Shinji (González et al. 1978; Starý et al. 2000; Rakhshani et al. 2006a); Acyrthosiphon pisum (Harris) (González et al. 1978; Starý 1979; Mescheloff \& Rosen 1990b; Starý et al. 2000; Rakhshani et al. 2006a, 2008a, 2012b; Nazari et al. 2012; Barahoei et al. 2013); Nearctaphis bakeri (Cowen) (Barahoei et al. 2013); Sitobion avenae (F.) (?) (Rakhshani et al. 2008a).

\section{Distribution in the Middle East and North Africa}

Algeria (Laamari et al. 2011); Iran (Starý 1979; Starý et al. 2000; Rakhshani et al. 2006a, 2008a, 2012b; Barahoei et al. 2012, 2013; Nazari et al. 2012); Iraq (Al-Azawi 1970); Israel (Mescheloff \& Rosen 1990b); Turkey (Western part - Akar \& Erdoğan 2017).

\section{General distribution}

Australasian, Holarctic, Neotropical, Oceanic, Oriental.

Aphidius sonchi Marshall, 1896

Figs 103, 206, 302, 403, 540

\section{Host records}

Hyperomyzus lactucae (L.) (Laamari et al. 2012; Ayadi et al. 2017)

\section{Distribution in the Middle East and North Africa}

Algeria (Laamari et al. 2012); Egypt (Hassan 1957; Starý 1976); Israel (Bodenheimer \& Swirski 1957; Starý 1976); Tunisia (Ayadi et al. 2017).

\section{General distribution}

Australasian, Eastern and Western Palaearctic, Oceanic, Oriental.

Aphidius stigmaticus Rakhshani \& Tomanović, 2011

Figs 21, 104, 207, 303, 404, 495, 541

\section{Host records}

Macrosiphoniella tanacetaria (Kaltenbach) (Rakhshani et al. 2011).

\section{Distribution in the Middle East and North Africa}

Iran (Rakhshani et al. 2011).

\section{General distribution}

This species has not been reported outside Iran. 
Aphidius transcaspicus Telenga, 1958

Figs 22, 105, 208, 304, 405, 496, 542

\section{Material examined}

SAUDI ARABIA • 9 우, 1 đ; Abha; May 2013; Hyalopterus pruni (Geoffroy) on Prunus armeniaca; Z. Ahmed leg. (DPPZ).

\section{Host records}

Aphis nasturtii Kaltenbach (?) (Starý \& Kaddou 1971 - as Aphis zizyphi Theobald); Brachycaudus amygdalinus (Schouteden) (?) (Mescheloff \& Rosen 1990b); Hyalopterus amygdali (Blanchard) (Aslan et al. 2004; Uysal et al. 2004; Rakhshani et al. 2008a; Mossadegh et al. 2011; Rakhshani 2012; Jafari et al. 2011; Jafari \& Modarres Awal 2012; Barahoei et al. 2013; Taheri \& Rakhshani 2013); Hyalopterus pruni (Geoffroy) (Starý \& Kaddou 1971; Erkin 1983; Mescheloff \& Rosen 1990b - as Aphidius magdae n.sp.; Mokhtari et al. 2000; Starý et al. 2000; Ölmez \& Ulusoy 2003; Aslan et al. 2004; Uysal et al. 2004; Lozier et al. 2008; Rakhshani et al. 2008a, 2012b; Jafari et al. 2011; Jafari \& Modarres Awal 2012; Laamari et al. 2011, 2012; Nazari et al. 2012; Rakhshani 2012; Tomanović et al. 2012; Barahoei et al. 2013; Taheri \& Rakhshani 2013; Ayadi et al. 2017); Melanaphis donacis (Passerini) (Starý \& Kaddou 1971; Mescheloff \& Rosen 1990b; Barahoei et al. 2014).

\section{Distribution in the Middle East and North Africa}

Algeria (Laamari et al. 2011, 2012); Iran (Starý et al. 2000; Mossadegh et al. 2011; Nazari et al. 2012; Rakhshani et al. 2008a, 2012b; Rakhshani 2012; Barahoei et al. 2013; Taheri \& Rakhshani 2013); Egypt (Lozier et al. 2008); Iraq (Al-Rawy et al. 1969; Starý 1969a, 1969c; Starý \& Kaddou 1971); Israel (Mescheloff \& Rosen 1990b; Lozier et al. 2008); Morocco (Lozier et al. 2008); Tunisia (Lozier et al. 2008; Ben Halima et al. 2013; Ayadi et al. 2017); Turkey (Uysal et al. 2004; Eastern part - Ölmez \& Ulusoy 2003; Lozier et al. 2008; Western part - Aslan et al. 2004; Erkin 1983; Erdoğan et al. 2008).

\section{General distribution}

Eastern and Western Palaearctic, Nearctic (imported), Oriental.

Aphidius uroleuci Mescheloff \& Rosen, 1990

Figs 106, 209, 305, 406, 497, 543

\section{Host records}

Uroleucon carthami (Hille Ris Lambers), Uroleucon inulae (Ferrari), Uroleucon jaceae (L.), Uroleucon sonchi (L.) (Mescheloff \& Rosen 1990b).

\section{Distribution in the Middle East and North Africa}

Israel (Mescheloff \& Rosen 1990b).

\section{General distribution}

This species has not been recorded outside Israel.

Aphidius urticae Haliday, 1834

Figs 23, 107, 210, 306, 407, 498, 544

\section{Host records}

Acyrthosiphon gossypii Mordvilko (Barahoei et al. 2013); Acyrthosiphon pisum (Harris) (Mescheloff \& Rosen 1990b; Rakhshani et al. 2008a); Acyrthosiphon sp. (Starý \& González 1978); Macrosiphum 
euphorbiae (Thomas) (Starý 1976); Microlophium carnosum (Buckton) (Starý et al. 2000; Talebi et al. 2009; Rakhshani et al. 2008a).

\section{Distribution in the Middle East and North Africa}

Iran (Starý et al. 2000; Rakhshani et al. 2008a; Barahoei et al. 2013); Israel (Mescheloff \& Rosen 1990b); Morocco (González et al. 1978); Turkey (Starý 1976; Eastern part - Tomanović et al. 2008; Western part - Tomanović et al. 2008; Akar \& Erdoğan 2017).

\section{General distribution}

Holarctic, Oceanic, Oriental.

Aphidius uzbekistanicus Luzhetzki, 1960

Figs 24, 108, 211, 307, 408, 499, 545

\section{Host records}

Diuraphis noxia (Kurdjumov) (Zareh et al. 1995; Starý et al. 2000); Metopolophium dirhodum (Walker) (Mescheloff \& Rosen 1990b; Özder \& Toros 1999; Aslan et al. 2004; Uysal et al. 2004; Rakhshani et al. 2008a, 2008b; Barahoei et al. 2013); Rhopalosiphum maidis (Fitch) (Ibrahim 1990a; El-Serafy 1999; Özder \& Toros 1999; Starý et al. 2000; Uysal et al. 2004; Gadallah et al. 2017); Rhopalosiphum padi (L.) (Starý 1979; Ibrahim 1990a; El-Serafy 1999; Starý et al. 2000; Sertkaya \& Yigit 2002; Aslan et al. 2004; Uysal et al. 2004; Rakhshani et al. 2008a, 2008b; Nazari et al. 2012; Gadallah et al. 2017); Schizaphis graminum (Rondani) (Ibrahim 1990a; El-Serafy 1999; Starý et al. 2000; Uysal et al. 2004; Rakhshani et al. 2008a, 2008b; Barahoei et al. 2013); Sitobion avenae (Fabricius) (Starý 1979; Mescheloff \& Rosen 1990b; Darvish-Mojeni \& Bayat-Asadi 1995; El-Serafy 1999; Özder \& Toros 1999; Starý et al. 2000; Sertkaya \& Yigit 2002; Ölmez \& Ulusoy 2003; Uysal et al. 2004; Rakhshani et al. 2008a, 2008b, 2012b; Barahoei et al. 2013; Gadallah et al. 2017); Sitobion fragariae (Walker) (Mescheloff \& Rosen 1990b; Laamari et al. 2011, 2012).

\section{Distribution in the Middle East and North Africa}

Algeria (Laamari et al. 2011, 2012); Egypt (Ibrahim 1990a; El-Serafy 1999; Gadallah et al. 2017); Iran (Starý 1979; Starý et al. 2000; Nazari et al. 2012; Rakhshani et al. 2008a, 2008b, 2012b; Barahoei et al. 2013); Israel (Mescheloff \& Rosen 1990b); Morocco (Starý 1981b); Turkey (Eastern part - Ölmez \& Ulusoy 2003; Central part - Aslan et al. 2004; Uysal et al. 2004; Western part - Özder \& Toros 1999; Sertkaya \& Yigit 2002; Erdoğan et al. 2008).

\section{General distribution}

Holarctic, Neotropical, Oriental.

$$
\text { Areopraon lepelleyi (Waterston, 1926) }
$$

Figs 25, 109, 212, 308, 409, 546

\section{Host records}

Eriosoma lanuginosum (Hartig) (Kazemzadeh et al. 2009; Rakhshani et al. 2012b).

\section{Distribution in the Middle East and North Africa}

Iran (Kazemzadeh et al. 2009; Rakhshani et al. 2012b).

\section{General distribution}

Eastern and Western Palaearctic, Oriental (India). 


\section{Betuloxys hortorum (Starý, 1960)}

Figs 110, 213, 309, 410, 547

\section{Host records}

Tinocallis saltans (Nevsky) (Mackauer \& Starý 1967; Starý 1979; Starý et al. 2000).

\section{Distribution in the Middle East and North Africa}

Iran (Mackauer \& Starý 1967; Starý 1979; Starý et al. 2000).

\section{General distribution}

Eastern Palaearctic (Iran), Western Palaearctic (Eastern and central Europe), Oriental (India).

Binodoxys acalephae (Marshall, 1896)

Figs 26, 111, 214, 310, 411, 548

\section{Host records}

Acyrthosiphon pisum (Harris) (?) (Talebi et al. 2009; Barahoei et al. 2013; Chaouche \& Laamari 2015); Anuraphis sp. (Güz \& Kilinçer 2005); Aphis affinis Del Guercio (Starý \& Kaddou 1971; Starý 1976); Aphis craccivora Koch (Starý 1979; Starý et al. 2000; Uysal et al. 2004; Rakhshani et al. 2005a, 2012b; Talebi et al. 2009; Laamari et al. 2011, 2012; Nazari et al. 2012; Barahoei et al. 2013; Ayadi et al. 2017); Aphis euphorbiae Kaltenbach (Talebi et al. 2009; Nazari et al. 2012); Aphis fabae Scopoli (Düzgüneş et al. 1982; Ölmez \& Ulusoy 2003; Uysal et al. 2004; Güz \& Kilinçer 2005; Talebi et al. 2009; Laamari et al. 2011, 2012; Rakhshani et al. 2012b; Nazari et al. 2012; Barahoei et al. 2013); Aphis fabae cirsiiacanthoidis Scopoli (Uysal et al. 2004); Aphis galiiscabri Schrank (Güz \& Kilinçer 2005); Aphis gossypii Glover (Yumruktepe \& Uygun 1994; Uysal et al. 2004; Laamari et al. 2011); Aphis grossulariae Kaltenbach (Starý 1976; Uysal et al. 2004); Aphis idaei van der Goot (Barahoei et al. 2014); Aphis nerii Boyer de Fonscolombe (Talebi et al. 2009); Aphis pomi de Geer (Rakhshani 2012); Aphis punicae Passerini (Ayadi et al. 2017); Aphis ruborum (Börner) (Uysal et al. 2004); Aphis vallei Hille Ris Lambers \& Stroyan (Starý 1976; Düzgüneş et al. 1982; Uysal et al. 2004); Aphis spiraecola Patch (Rakhshani 2012); Aphis umbrella (Börner) (Mackauer \& Starý 1967; Starý et al. 2000; Talebi et al. 2009; Nazari et al. 2012); Aphis urticata Gmelin (Talebi et al. 2009); Aphis sp. (Starý 1976; 1979; Mossadegh et al. 2011); Hyadaphis coriandri (Das) (Chaouche \& Laamari 2015); Protaphis sp. (Barahoei et al. 2012, 2013).

\section{Distribution in the Middle East and North Africa}

Algeria (Laamari et al. 2011, 2012; Chaouche \& Laamari 2015); Egypt (El-Heneidy 1994; AbdelRahman et al. 2000; El-Heneidy \& Abdel-Samad 2001; El-Heneidy et al. 2001, 2002, 2003, 2004); Iran (Starý 1979; Starý et al. 2000; Rakhshani et al. 2005a; Mossadegh et al. 2011; Barahoei et al. 2012, 2013; Nazari et al. 2012; Rakhshani 2012; Rakhshani et al. 2012b); Iraq (Starý \& Kaddou 1971; Starý 1976); Morocco (Starý 1976); Tunisia (Ayadi et al. 2017); Turkey (Starý 1976; Ölmez \& Ulusoy 2003; Uysal et al. 2004; Central part - Güz \& Kilinçer 2005; Western part - Akar \& Erdoğan 2017); UAE (Starý et al. 2013); Yemen (Starý et al. 2013).

\section{General distribution}

Holarctic, Oriental. 
RAKHSHANI E. et al., Aphidiinae of the Middle East and North Africa

Binodoxys angelicae (Haliday, 1833)

Figs 27, 112, 215, 311, 412, 549

\section{Host records}

Aphis acetosae L. (Barahoei et al. 2013); Aphis affinis del Guercio (Mossadegh et al. 2011; Barahoei et al. 2013; Taheri \& Rakhshani 2013); Aphis aurantii Boyer de Fonscolombe (Rosen 1967, 1969; Avidov \& Harpaz 1969; Starý 1976; Hussein \& Kawar 1984; Tremblay et al. 1985; Mescheloff \& Rosen 1993; Ben Halima \& Ben Hamouda 2005; Boukhris-Bouhachem 2011; Yoldaş et al. 2011); Aphis craccivora Koch (Avidov \& Harpaz 1969; Starý \& Kaddou 1971; Starý 1976; Düzgüneş et al. 1982; Abou-Fakhr 1982; Tremblay et al. 1985; Mescheloff \& Rosen 1993; Ragab 1996; Abou-Fakhr \& Kawar 1998; Ölmez \& Ulusoy 2003; Aslan et al. 2004; Uysal et al. 2004; Rakhshani et al. 2005a; Yoldaş et al. 2011; Laamari et al. 2011, 2012; Mossadegh et al. 2011; Nazari et al. 2012; Barahoei et al. 2013; Satar et al. 2014); Aphis epilobiaria Theobald (Mescheloff \& Rosen 1993; Nazari et al. 2012); Aphis fabae Scopoli (Al-Azawi 1970; Starý 1976; Abou-Fakhr 1982; Hussein \& Kawar 1984; Tremblay et al. 1985; Mescheloff \& Rosen 1993; Ben Halima et al. 1994 - as Aphis citricola van der Goot; Abou-Fakhr \& Kawar 1998; Ölmez \& Ulusoy 2003; Uysal et al. 2004; Ben Halima \& Ben Hamouda 2005 - as Aphis citricola van der Goot; Güz \& Kilinçer 2005; Talebi et al. 2009; Laamari et al. 2011, 2012; Mossadegh et al. 2011; Barahoei et al. 2012, 2013; Nazari et al. 2012; Taheri \& Rakhshani 2013); Aphis fabae cirsiiacanthoidis Scopoli (Aslan et al. 2004; Uysal et al. 2004); Aphis farinosa Gmelin (Starý et al. 2000); Aphis gossypii Glover (Mimeur 1934; Rosen 1967, 1969; Avidov \& Harpaz 1969; Al-Azawi 1970; Starý 1976; Abou-Fakhr 1982; Zeren \& Düzgüneş 1983; Hussein \& Kawar 1984; Tremblay et al. 1985; Mescheloff \& Rosen 1993; Ben Halima \& Ben Hamouda 1993; Yumruktepe \& Uygun 1994; Abou-Fakhr \& Kawar 1998; Starý et al. 2000; Yoldaş et al. 2002; Uysal et al. 2004; Mossadegh et al. 2011; Yoldaş et al. 2011; Laamari et al. 2011, 2012; Nazari et al. 2012; Barahoei et al. 2013; Satar et al. 2014; Ayadi et al. 2017); Aphis hederae Kaltenbach (Abou-Fakhr 1982; Tremblay et al. 1985; Mescheloff \& Rosen 1993; Abou-Fakhr \& Kawar 1998; Starý et al. 2000); Aphis idaei van der Goot (Barahoei et al. 2013; Taheri \& Rakhshani 2013); Aphis intybi Koch (Mescheloff \& Rosen 1993); Aphis nasturtii Kaltenbach (Starý \& Kaddou 1971 - as Aphis zizyphi Theobald; Starý 1976; Mescheloff \& Rosen 1993); Aphis nerii Boyer de Fonscolombe (Starý 1976; Mescheloff \& Rosen 1993; Ben Halima et al. 1994; Ben Halima \& Ben Hamouda 2005; Talebi et al. 2009; Laamari et al. 2011); Aphis pomi de Geer (Erkin 1983; Hussein \& Kawar 1984; Radjabi 1989; Mescheloff \& Rosen 1993; Starý et al. 2000; Uysal et al. 2004; Rakhshani 2012); Aphis polygonata (Nevsky) (Mossadegh et al. 2011); Aphis punicae Passerini (Starý 1976; Al-Azawi 1970; Starý \& Kaddou 1971; Mescheloff \& Rosen 1993; Ölmez \& Ulusoy 2003; Uysal et al. 2004; Laamari et al. 2011, 2012; Ayadi et al. 2017); Aphis ruborum (Börner) (Düzgüneş et al. 1982; Aslan et al. 2004; Uysal et al. 2004); Aphis rumicis L. (Talebi et al. 2009); Aphis solanella Theobald (Starý \& Kaddou 1971; Starý 1976; Güz \& Kilinçer 2005; Barahoei et al. 2012, 2013; Rakhshani 2012; Ayadi et al. 2017); Aphis spiraecola Patch (Mescheloff \& Rosen 1993; Boukhris-Bouhachem 2011; Yoldaş et al. 2011; Labdaoui \& Guenaoui 2018); Aphis umbrella (Börner) (Mackauer \& Starý 1967; Abou-Fakhr 1982; Tremblay et al. 1985; Mescheloff \& Rosen 1993; AbouFakhr \& Kawar 1998; Starý et al. 2000; Talebi et al. 2009; Laamari et al. 2011, 2012; Mossadegh et al. 2011; Nazari et al. 2012); Aphis viticis Ferrari (Starý \& Kaddou 1971; Starý 1976); Aphis sp. (Mimeur 1934; Starý 1976; Uysal et al. 2004; Laamari et al. 2012); Brachycaudus helichrysi (Kaltenbach) (Satar et al. 2014); Myzus persicae (Sulzer) (Hussein \& Kawar 1984; Tremblay et al. 1985; Satar et al. 2014); Sitobion avenae (Fabricius) (?) (Gadallah et al. 2017).

\section{Distribution in the Middle East and North Africa}

Algeria (Starý 1976; Laamari et al. 2011, 2012; Labdaoui \& Guenaoui 2018); Egypt (Mackauer \& Starý 1967; Starý 1976; Ragab 1996; Gadallah et al. 2017); Iran (Starý et al. 2000; Rakhshani et al. 2005a; Mossadegh et al. 2011; Barahoei et al. 2012, 2013; Nazari et al. 2012; Rakhshani 2012; Taheri \& 
Rakhshani 2013); Iraq (Starý 1969a; Al-Azawi 1970; Starý \& Kaddou 1971; Starý 1976); Israel (Rosen 1967, 1969; Avidov \& Harpaz 1969; Starý 1976; Mescheloff \& Rosen 1993); Lebanon (Mackauer \& Starý 1967; Starý 1976; Abou-Fakhr 1982; Hussein \& Kawar 1984; Tremblay et al. 1985; Abou-Fakhr \& Kawar 1998); Morocco (Mimeur 1934; Starý 1976); Tunisia (Mackauer \& Starý 1967; Starý 1976; Ben Halima \& Ben Hamouda 1993, 2005; Ben Halima et al. 1994; Boukhris-Bouhachem 2011; Ayadi et al. 2017); Turkey (Uysal et al. 2004; Eastern part - Ölmez \& Ulusoy 2003; Central part - Zeren \& Düzgüneş 1983; Düzgüneş et al. 1982; Aslan et al. 2004; Güz \& Kilinçer 2005; Yumruktepe \& Uygun 1994; Satar et al. 2014; Western part - Erkin 1983; Aslan \& Karaca 2005; Erdoğan et al. 2008; Yoldaş et al. 2011; Akar \& Erdoğan 2017); UAE (Starý et al. 2013).

\section{General distribution}

Eastern and Western Palaearctic, Oriental.

\section{Binodoxys brevicornis (Haliday, 1833)}

Figs 28, 113, 216, 312, 413, 550

\section{Host records}

Cavariella aegopodii (Scopoli) (Starý 1976; Mescheloff \& Rosen 1993; Rakhshani et al. 2007b; Talebi et al. 2009; Barahoei et al. 2013); Hyadaphis coriandri (Das) (Mescheloff \& Rosen 1993; Düzgüneş et al. 1982; Uysal et al. 2004; Ayadi et al. 2017); Hyadaphis foeniculi (Passerini) (Starý 1976); Hyadaphis sp. (Starý et al. 2000; Güz \& Kilinçer 2005).

\section{Distribution in the Middle East and North Africa}

Algeria (Starý 1976); Iran (Starý et al. 2000; Rakhshani et al. 2007b; Barahoei et al. 2013); Israel (Starý 1976; Mescheloff \& Rosen 1993); Tunisia (Ayadi et al. 2017); Turkey (Starý 1976; Central part - Düzgüneş et al. 1982; Uysal et al. 2004; Güz \& Kilinçer 2005).

\section{General distribution}

Eastern and Western Palaearctic, Neotropical (accidentally introduced), Oriental (India).

\section{Binodoxys centaureae (Haliday, 1833)}

Figs 114, 217, 313, 414, 551

\section{Host records}

Uroleucon sp. (Starý 1976; Laamari et al. 2012).

\section{Distribution in the Middle East and North Africa}

Algeria (Starý 1976; Laamari et al. 2012).

\section{General distribution}

Eastern and Western Palaearctic, Oriental.

Binodoxys heraclei (Haliday, 1833)

Figs 29, 115, 218, 314, 415, 552

\section{Host records}

Cavariella aspidaphoides Hille Ris Lambers (Rakhshani et al. 2007b; Talebi et al. 2009; Nazari et al. 2012); Cavariella theobaldi (Gillette \& Bragg) (Düzgüneş et al. 1982; Uysal et al. 2004). 
RAKHSHANI E. et al., Aphidiinae of the Middle East and North Africa

\section{Distribution in the Middle East and North Africa}

Iran (Rakhshani et al. 2007b; Talebi et al. 2009; Nazari et al. 2012); Turkey (Central part - Düzgüneş et al. 1982; Uysal et al. 2004).

\section{General distribution}

Eastern and Western Palaearctic, Oriental.

\section{Host records}

Binodoxys sp.

Lacking host data (Samara \& Qubbaj 2012).

\section{Distribution in the Middle East and North Africa}

Palestine (Samara \& Qubbaj 2012).

Diaeretiella rapae (M'Intosh, 1855)

Figs 30, 116, 219, 315, 416, 553

\section{Material examined}

SAUDI ARABIA • 1 क; Abha; agricultural field; 28 Nov. 2012; Brevicoryne brassicae (L.) on Brassica

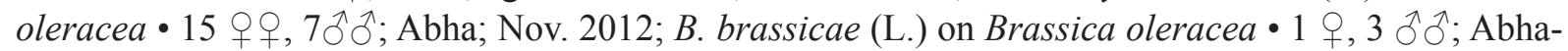
Mahala College campus; Nov. 2012; on Hordeum 14 웅, $5 \overbrace{}^{\widehat{\lambda}}$; Abha; May 2013; B. brassicae (L.) on Raphanus sativus $\bullet 6$ 우; Abha; Jun. 2012; on Trifolium alexandrinum; Z. Ahmed leg. (DPPZ).

\section{Host records}

Amegosiphon platicaudum (Narzikulov) (Nazari et al. 2012); Aphis aurantii Boyer de Fonscolombe (Tremblay et al. 1985); Aphis craccivora Koch (Mescheloff \& Rosen 1990b; Rakhshani et al. 2006a; Nazari et al. 2012; Taheri \& Rakhshani 2013; Barahoei et al. 2013; Ayadi et al. 2017); Aphis fabae Scopoli (Abou-Fakhr 1982; Tremblay et al. 1985; Güz \& Kilinçer 2005; Laamari et al. 2012; Barahoei et al. 2013); Aphis gossypii Glover (Ben Halima \& Ben Hamouda 1993; Laamari et al. 2011, 2012; Mossadegh et al. 2011; Barahoei et al. 2013; Satar et al. 2014); Aphis illinoisensis Shimer (El-Gantiry et al. 2012); Aphis nerii Boyer de Fonscolombe (Laamari et al. 2012); Aphis punicae Passerini (Nazari et al. 2012); Aphis solanella Theobald, Aphis umbrella (Börner) (Barahoei et al. 2013); Aphis spiraecola Patch (Laamari et al. 2012); Aspidaphis adjuvans (Walker) (Mescheloff \& Rosen 1990b); Brachycaudus amygdalinus (Schouteden) (Starý 1979; Mescheloff \& Rosen 1990b; Starý et al. 2000); Brachycaudus cardui (L.) (Starý 1979; Starý et al. 2000; Laamari et al. 2011, 2012); Brachycaudus helichrysi (Kaltenbach) (Erkin 1983; Uysal et al. 2004; Laamari et al. 2011, 2012; Chaouche \& Laamari 2015); Brevicoryne brassicae (L.) (Talhouk 1961; Hafez 1965; Davatchi \& Shojai 1968; Al-Azawi 1970; Starý 1976, 1979; Abou-Fakhr 1982; Kilinçer 1982; Düzgüneş et al. 1982; Zeren \& Düzgüneş 1983; Hussein \& Kawar 1984; Tremblay et al. 1985; Starý \& Sekkat 1987; Mescheloff \& Rosen 1990b; Avci \& Özbek 1991; Abou-Fakhr \& Kawar 1998; Starý et al. 2000; Ölmez \& Ulusoy 2003; Aslan et al. 2004; Uysal et al. 2004; Güz \& Kilinçer 2005; Talebi et al. 2009; Laamari et al. 2011, 2012; Mossadegh et al. 2011; Barahoei et al. 2012, 2013; Nazari et al. 2012; Rakhshani et al. 2012b; Taheri \& Rakhshani 2013; Chaouche \& Laamari 2015); Capitophorus inulae (Passerini) (Mescheloff \& Rosen 1990b); Diuraphis noxia (Kurdjumov) (Starý 1976; González et al. 1992; Ahmadi \& Sarafrazi 1993; Elmali \& Toros 1994; Zareh et al. 1995; Ahmadi 2000; Starý et al. 2000; Baer et al. 2004; Uysal et al. 2004; Rakhshani et al. 2008b, 2012b; Laamari et al. 2011, 2012; Nazari et al. 2012; Barahoei et al. 2013; Laamari et al. 2016); Dysaphis foeniculus (Theobald) (Ayadi et al. 2017); Dysaphis pyri (Boyer de Fonscolombe) (Erkin 1983; Uysal et al. 2004); Dysaphis tulipae (Boyer de Fonscolombe) (Starý 
1976; Laamari et al. 2011, 2012); Hayhurstia atriplicis (L.) (Starý 1976, 1979; Mescheloff \& Rosen 1990b; Starý et al. 2000; Güz \& Kilinçer 2005; Laamari et al. 2012; Nazari et al. 2012; Rakhshani et al. 2012b); Hyadaphis coriandri (Das) and Hyadaphis foeniculi (Passerini) (Güz \& Kilinçer 2005); Hyadaphis tataricae (Aizenberg) (Aslan et al. 2004; Uysal et al. 2004); Hyperomyzus lactucae (L.) (Güz \& Kilinçer 2005); Lipaphis erysimi (Kaltenbach) (Starý 1979; Starý et al. 2000; Ölmez \& Ulusoy 2003; Uysal et al. 2004; Mossadegh et al. 2011); Lipaphis lepidii (Nevsky) (Starý et al. 2000; Talebi et al. 2009; Mossadegh et al. 2011; Nazari et al. 2012); Lipaphis pseudobrassicae (Davis) (Barahoei et al. 2013); Lipaphis sp. (Güz \& Kilinçer 2005); Macrosiphoniella sanborni (Gillette) (Al-Azawi 1970; Starý 1976); Neomariaella lambersi (Szelegiewicz) (Starý 1979; Starý et al. 2000); Metopolophium dirhodum (Walker) (Rakhshani et al. 2008b); Myzus beybienkoi (Narzykulov) (Starý 1979; Starý et al. 2000); Myzus persicae (Sulzer) (Avidov \& Kotter 1966; Avidov \& Harpaz 1969; Starý 1976; Starý 1979; Erkin 1983; Starý \& Sekkat 1987; Hodjat \& Moradeshaghi 1988; Mescheloff \& Rosen 1990b; Starý et al. 2000; Boukhris-Bouhachem 2011; Laamari et al. 2011; Mossadegh et al. 2011; Jafari et al. 2011; Jafari \& Modarres Awal 2012; Nazari et al. 2012; Rakhshani 2012; Taheri \& Rakhshani 2013; Barahoei et al. 2013; Chaouche \& Laamari 2015); Rhopalosiphum maidis (Fitch) (Mimeur 1934; Starý 1976, 1979; Starý \& Sekkat 1987; El-Heneidy \& Attia 1988/1989; El-Heneidy 1991, 1994; Elmali \& Toros 1994; Starý et al. 2000; El-Heneidy \& Abdel-Samad 2001; El-Heneidy et al. 2001, 2002; Uysal et al. 2004; Slman 2006; Laamari et al. 2011, 2012; Barahoei et al. 2013; Ayadi et al. 2017); Rhopalosiphum padi (L.) (Hassan 1963; El-Heneidy \& Attia 1988/1989; El-Heneidy 1991, 1994; Starý et al. 2000; ElHeneidy \& Abdel-Samad 2001; El-Heneidy et al. 2001, 2002; Uysal et al. 2004; Abdel-Rahman 2005; Slman 2006; Rakhshani et al. 2008b; Mossadegh et al. 2011; Barahoei et al. 2013); Saltusaphis scirpus Theobald (Starý et al. 2000); Schizaphis graminum (Rondani) (Bodenheimer \& Swirski 1957; Starý 1976, 1979; El-Heneidy \& Attia 1988/1989; El-Heneidy 1991, 1994; Starý et al. 2000; El-Heneidy \& Abdel-Samad 2001; El-Heneidy et al. 2001, 2002; Abdel-Rahman 2005; Slman 2006; Rakhshani et al. 2008b; Barahoei et al. 2013); Sitobion avenae (Fabricius) (El-Heneidy \& Attia 1988/1989; El-Heneidy 1991, 1994; Starý et al. 2000; El-Heneidy et al. 2001, 2002; Slman 2006; Rakhshani et al. 2008b; Mossadegh et al. 2011; Rakhshani et al. 2012b; Barahoei et al. 2013); Uroleucon sonchi (L.) (?) (Starý \& Kaddou 1971).

\section{Distribution in the Middle East and North Africa}

Algeria (Starý 1976; Laamari et al. 2011, 2012; Chaouche \& Laamari 2015); Egypt (Hassan 1963; Hafez 1965; Mackauer \& Starý 1967; Starý 1976; El-Heneidy \& Attia 1988/1989; El-Heneidy 1991, 1994; El-Heneidy \& Abdel-Samad 2001; El-Heneidy et al. 2001, 2002; Ragab et al. 2002; Abdel-Rahman 2005; Slman 2006; El-Gantiry et al. 2012); Iran (Starý 1979; Starý et al. 2000; Mossadegh et al. 2011; Barahoei et al. 2012, 2013; Nazari et al. 2012; Rakhshani et al. 2006a, 2012b; Rakhshani 2012; Taheri \& Rakhshani 2013); Iraq (Al-Azawi 1970; Starý \& Kaddou 1971; Starý 1976); Israel (Bodenheimer \& Swirski 1957; Avidov \& Kotter 1966; Avidov \& Harpaz 1969; Starý 1976; Mescheloff \& Rosen 1990b); Jordan (Baer et al. 2004); Lebanon (Talhouk 1961; Starý 1976; Abou-Fakhr 1982; Hussein \& Kawar 1984; Tremblay et al. 1985; Abou-Fakhr \& Kawar 1998); Libya (Mackauer \& Starý 1967; Starý 1976); Morocco (Mimeur 1934; Starý 1976; Starý \& Sekkat 1987; Baer et al. 2004); Palestine (Samara \& Qubbaj 2012); Saudi Arabia (Ahmad \& Bakr 2013); Syria (Baer et al. 2004); Tunisia (Ben Halima \& Ben Hamouda 1993; Boukhris-Bouhachem 2011; Ayadi et al. 2017); Turkey (Eastern part - Ölmez \& Ulusoy 2003; Central part - Düzgüneş et al. 1982, Kilinçer 1982; Zeren \& Düzgüneş 1983; Elmali \& Toros 1994; Aslan et al. 2004; Uysal et al. 2004; Güz \& Kilinçer 2005; Satar et al. 2014; Western part Erkin 1983; Erdoğan et al. 2008; Akar \& Erdoğan 2017); Yemen (Starý et al. 2013).

\section{General distribution}

Cosmopolitan. 


\section{Diaeretus leucopterus (Haliday, 1834)}

Figs 117, 220, 316, 417, 554

\section{Host records}

Eulachnus tuberculostemmatus (Theobald) (Starý 1976; Mescheloff \& Rosen 1990b).

\section{Distribution in the Middle East and North Africa}

Israel (Starý 1976; Mescheloff \& Rosen 1990b).

\section{General distribution}

Eastern and Western Palaearctic, Oriental.

Ephedrus cerasicola Starý, 1962

Figs 31, 118, 221, 317, 418, 555

\section{Host records}

Dysaphis plantaginea (Passerini) (Rakhshani 2012; Barahoei et al. 2013); Myzus cerasi (F.) (Rakhshani 2012); Myzus persicae (Sulzer) (Düzgüneş et al. 1982; Uysal et al. 2004; Rakhshani 2012); Phorodon humuli (Schrank) (Mokhtari et al. 2000; Starý et al. 2000); Rhopalosiphum nymphaeae (L.) (Mokhtari et al. 2000; Starý et al. 2000).

\section{Distribution in the Middle East and North Africa}

Iran (Starý et al. 2000; Rakhshani 2012; Barahoei et al. 2013); Turkey (Central part - Düzgüneş et al. 1982; Uysal et al. 2004).

\section{General distribution}

Holarctic, Oceanic.

Ephedrus chaitophori Gärdenfors, 1986

Figs 119, 222, 318, 419, 556

\section{Host records}

Chaitophorus populeti (Panzer) (Rakhshani et al. 2007b).

\section{Distribution in the Middle East and North Africa}

Iran (Rakhshani et al. 2007b).

\section{General distribution}

Holarctic.

Ephedrus helleni Mackauer, 1968

Figs 32, 120, 223, 319, 420, 557

\section{Host records}

Cavariella aquatica (Gillette \& Bragg) (Starý 1979; Starý et al. 2000; Rakhshani et al. 2007b; Talebi et al. 2009); Hayhurstia atriplicis (L.) (Barahoei et al. 2014). 


\section{Distribution in the Middle East and North Africa}

Iran (Starý 1979; Starý et al. 2000; Rakhshani et al. 2007b; Talebi et al. 2009).

\section{General distribution}

Eastern and Western Palaearctic, Oriental.

\section{Ephedrus lacertosus (Haliday, 1833)}

Figs 121, 224, 320, 421, 558

\section{Host records}

Lacking host data (Tomanović et al. 2008).

\section{Distribution in the Middle East and North Africa}

Turkey (Eastern part - Tomanović et al. 2008).

\section{General distribution}

Holarctic, Neotropical, Oriental.

Ephedrus nacheri Quilis, 1934

Figs 122, 225, 321, 422, 559

\section{Host records}

Hayhurstia atriplicis (L.) (Düzgüneş et al. 1982; Uysal et al. 2004).

\section{Distribution in the Middle East and North Africa}

Turkey (Central part - Düzgüneş et al. 1982; Uysal et al. 2004).

\section{General distribution}

Eastern and Western Palaearctic, Oriental.

Ephedrus niger Gautier, Bonnamour \& Gaumont, 1929

Figs 33, 123, 226, 322, 423, 560

\section{Host records}

Macrosiphoniella abrotani (Walker) (Mackauer \& Starý 1967; Starý et al. 2000; Rakhshani et al. 2011); Macrosiphoniella sanborni (Gillette) (Rakhshani et al. 2011; Nazari et al. 2012); Macrosiphoniella sp. (Starý 1979; Starý et al. 2000; Rakhshani et al. 2011; Barahoei et al. 2013); Macrosiphum rosae (L.) (?) (Mescheloff \& Rosen 1988); Uroleucon acroptilidis Kadyrbekov, Renxin \& Shao, Uroleucon bielawskii (Szelegiewicz) (Nazari et al. 2012; Barahoei et al. 2013); Uroleucon cichorii (Koch) (Starý et al. 2000; Barahoei et al. 2012, 2013); Uroleucon compositae (Theobald) (Nazari et al. 2012; Barahoei et al. 2013); Uroleucon erigeronense (Thomas) (Starý et al. 2000; Talebi et al. 2009; Nazari et al. 2012); Uroleucon inulae (Ferrari) (Mescheloff \& Rosen 1988); Uroleucon jaceae (L.) (Starý et al. 2000; Talebi et al. 2009; Rakhshani et al. 2011, 2012b; Barahoei et al. 2012, 2013; Nazari et al. 2012); Uroleucon sonchi (L.) (Talebi et al. 2009; Rakhshani et al. 2012b; Barahoei et al. 2012, 2013; Nazari et al. 2012; Taheri \& Rakhshani 2013); Uroleucon sp. (Starý 1979; Starý et al. 2000; Barahoei et al. 2012, 2013). 
RAKHSHANI E. et al., Aphidiinae of the Middle East and North Africa

\section{Distribution in the Middle East and North Africa}

Algeria (Gärdenfors 1986; Laamari et al. 2011); Iran (Mackauer \& Starý 1967; Starý 1979; Starý et al. 2000; Talebi et al. 2009; Barahoei et al. 2012, 2013; Nazari et al. 2012; Rakhshani et al. 2012b; Taheri \& Rakhshani 2013); Israel (Mescheloff \& Rosen 1988).

\section{General distribution}

Eastern and Western Palaearctic, Oriental.

Ephedrus persicae Froggatt, 1904

Figs 34, 124, 227, 323, 424, 561

\section{Material examined}

SAUDI ARABIA • 1 §̊; Raidah; Apr. 2011; net sweeping; Z. Ahmad leg. (DPPZ).

\section{Host records}

Absinthaphis sp. (Mescheloff \& Rosen 1988); Acyrthosiphon malvae (Mosley) (Laamari et al. 2011, 2012); Acyrthosiphon pisum (Harris) (Laamari et al. 2011); Aphis affinis del Guercio (Talebi et al. 2009; Barahoei et al. 2013; Taheri \& Rakhshani 2013); Aphis aurantii Boyer de Fonscolombe (Rosen 1967, 1969; Avidov \& Harpaz 1969; Starý 1976; Hussein \& Kawar 1984; Tremblay et al. 1985; Boukhris-Bouhachem 2011; Yoldaş et al. 2011); Aphis brunnea Ferrari, Aphis chloris Koch (Mescheloff \& Rosen 1988); Aphis craccivora Koch (Mackauer 1963; Avidov \& Harpaz 1969; Starý 1976; Mescheloff \& Rosen 1988; Düzgüneş et al. 1982; Starý et al. 2000; Uysal et al. 2004; Rakhshani et al. 2005a, 2006a; Žikić et al. 2009; Laamari et al. 2011; Yoldaş et al. 2011; Barahoei et al. 2012, 2013; Nazari et al. 2012); Aphis epilobiaria Theobald (Mescheloff \& Rosen 1988); Aphis fabae Scopoli (Al-Azawi 1970; Starý 1976, 1979; Düzgüneş et al. 1982; Tremblay et al. 1985; Mescheloff \& Rosen 1988; Uysal et al. 2004; Žikić et al. 2009; Laamari et al. 2011, 2012; Barahoei et al. 2013; Taheri \& Rakhshani 2013); Aphis fabae cirsiiacanthoidis Scopoli (Uysal et al. 2004); Aphis gossypii Glover (Al-Azawi 1970; Starý 1976; Abou-Fakhr 1982; Düzgüneş et al. 1982; Zeren \& Düzgüneş 1983; Tremblay et al. 1985; Mescheloff \& Rosen 1988; Abou-Fakhr \& Kawar 1998; Uysal et al. 2004; Yoldaş et al. 2011; Laamari et al. 2011, 2012; Barahoei et al. 2013; Satar et al. 2014; Ayadi et al. 2017); Aphis hederae Kaltenbach, Aphis intybi Koch (Mescheloff \& Rosen 1988); Aphis nerii Boyer de Fonscolombe (Talebi et al. 2009; Barahoei et al. 2014); Aphis plantaginis Goeze (Barahoei et al. 2013); Aphis pomi De Geer (Düzgüneş et al. 1982; Starý et al. 2000; Uysal et al. 2004; Laamari et al. 2011, 2012); Aphis punicae Passerini (Mackauer 1963; Starý \& Sekkat 1987; Mescheloff \& Rosen 1988; Starý et al. 2000; Laamari et al. 2011, 2012); Aphis ruborum (Börner) (Starý 1976; Düzgüneş et al. 1982; Uysal et al. 2004); Aphis solanella Theobald (Tremblay et al. 1985); Aphis umbrella (Börner) (Starý 1976; Abou-Fakhr 1982; Tremblay et al. 1985; AbouFakhr \& Kawar 1998); Aphis verbasci Schrank, Aphis nasturtii Kaltenbach (Mescheloff \& Rosen 1988 - as Aphis zizyphi Theobald); Brachycaudus amygdalinus (Schouteden) (Bodenheimer \& Swirski 1957; Avidov \& Harpaz 1969; Starý 1976; Starý \& Sekkat 1987; Düzgüneş et al. 1982; Mescheloff \& Rosen 1988; Ölmez \& Ulusoy 2003; Uysal et al. 2004; Talebi et al. 2009; Žikić et al. 2009; Jafari et al. 2011; Laamari et al. 2011; Jafari \& Modarres Awal 2012; Nazari et al. 2012; Rakhshani 2012; Rakhshani et al. 2012b); Brachycaudus cardui (L.) (Düzgüneş et al. 1982; Uysal et al. 2004; Aslan \& Karaca 2005; Žikić et al. 2009); Brachycaudus helichrysi (Kaltenbach) (Starý 1979; Erkin 1983; Mescheloff \& Rosen 1988; Starý et al. 2000; Uysal et al. 2004; Žikić et al. 2009; Laamari et al. 2011; Nazari et al. 2012; Rakhshani 2012; Barahoei et al. 2013; Satar et al. 2014); Brachyunguis tamaricis (Lichtenstein) (Starý 1976, 1979; Starý et al. 2000; Laamari et al. 2012); Cavariella aegopodii (Scopoli); Cavariella theobaldi (Gillette \& Bragg) (Düzgüneş et al. 1982; Uysal et al. 2004); Diuraphis noxia (Kurdjumov) (Starý et al. 2000); Dysaphis crataegi 
(Kaltenbach) (Talhouk 1961; Starý 1976; Starý et al. 2000); Dysaphis devecta (Walker) (Aslan et al. 2004; Uysal et al. 2004; Aslan \& Karaca 2005; Žikić et al. 2009); Dysaphis foeniculus (Theobald) (Talebi et al. 2009); Dysaphis plantaginea (Passerini) (Düzgüneş et al. 1982; Erkin 1983; Hussein \& Kawar 1984 - as Dysaphis mali (Ferrari); Starý et al. 2000; Uysal et al. 2004; Aslan \& Karaca 2005; Laamari et al. 2011, 2012; Nazari et al. 2012; Rakhshani 2012; Barahoei et al. 2013); Dysaphis pyri (Boyer de Fonscolombe) (Mackauer 1963; Erkin 1983; Starý et al. 2000; Uysal et al. 2004; Žikić et al. 2009; Rakhshani 2012); Dysaphis reaumuri (Mordvilko) (Rakhshani 2012; Barahoei et al. 2013); Eulachnus tuberculostemmatus (Theobald) (?) (Mescheloff \& Rosen 1988); Hayhurstia atriplicis (L.) (Mescheloff \& Rosen 1988; Barahoei et al. 2013); Hyadaphis coriandri (Das) (Talebi et al. 2009; Barahoei et al. 2014); Hyadaphis foeniculi (Passerini) (Mescheloff \& Rosen 1988); Hyalopterus amygdali (Blanchard) (Ölmez \& Ulusoy 2003; Aslan et al. 2004; Uysal et al. 2004; Jafari et al. 2011; Jafari \& Modarres Awal 2012; Rakhshani 2012; Barahoei et al. 2013); Hyalopterus pruni (Geoffroy) (Düzgüneş et al. 1982; Erkin 1983; Mescheloff \& Rosen 1988; Uysal et al. 2004; Aslan \& Karaca 2005); Macchiatiella rhamni (Boyer de Fonscolombe) (Abou-Fakhr 1982; AbouFakhr \& Kawar 1998); Melanaphis donacis (Passerini) (Mescheloff \& Rosen 1988); Metopolophium dirhodum (Walker) (Starý et al. 2000; Rakhshani et al. 2008b); Myzus cerasi (Fabricius) (Erkin 1983; Aslan et al. 2004; Uysal et al. 2004; Žikić et al. 2009; Rakhshani 2012); Myzus persicae (Sulzer) (Starý 1974; Erkin 1983; Zeren \& Düzgüneş 1983; Karaat \& Goven 1986; Uysal et al. 2004; Talebi et al. 2009; Žikić et al. 2009; Boukhris-Bouhachem 2011; Yoldaş et al. 2011; Laamari et al. 2011; Nazari et al. 2012; Rakhshani 2012; Barahoei et al. 2013; Hasan 2016); Phorodon humuli (Schrank) (Rakhshani 2012; Barahoei et al. 2013); Rhopalosiphum maidis (Fitch) (Avidov \& Harpaz 1969; Starý 1976, 1979; Gärdenfors 1986; Starý et al. 2000; El-Heneidy \& Abdel-Samad 2001; El-Heneidy et al. 2001, 2002; Mossadegh et al. 2011; Barahoei et al. 2012, 2013); Rhopalosiphum padi (L.) (Starý 1979; Gärdenfors 1986; Özder \& Toros 1999; Starý et al. 2000; El-Heneidy \& Abdel-Samad 2001; El-Heneidy et al. 2001, 2002; Uysal et al. 2004; Rakhshani et al. 2008b; Gadallah et al. 2017); Schizaphis graminum (Rondani) (Starý et al. 2000; El-Heneidy \& Abdel-Samad 2001; El-Heneidy et al. 2001, 2002; Rakhshani et al. 2008b; Žikić et al. 2009; Rakhshani et al. 2012b); Sipha maydis Passerini (?) (Starý et al. 2000); Sitobion avenae (Fabricius) (Starý et al. 2000; El-Heneidy et al. 2001, 2002; Barahoei et al. 2013; Gadallah et al. 2017).

\section{Distribution in the Middle East and North Africa}

Algeria (Starý 1976; Gärdenfors 1986; Laamari et al. 2011, 2012); Egypt (Gärdenfors, 1986; ElHeneidy \& Abdel-Samad 2001; El-Heneidy et al. 2001, 2002, 2003; El-Ghiet et al. 2014); Iran (Starý 1979; Starý et al. 2000; Rakhshani et al. 2005a, 2006a, 2008b, 2012b; Mossadegh et al. 2011; Barahoei et al. 2012, 2013; Nazari et al. 2012; Rakhshani 2012; Taheri \& Rakhshani 2013); Iraq (Al-Azawi 1970; Starý 1976; Žikić et al. 2009); Israel (Rosen 1967, 1969; Bodenheimer \& Swirski 1957; Avidov \& Harpaz 1969; Starý 1974, 1976; Gärdenfors, 1986; Mescheloff \& Rosen 1988); Jordan (Hasan 2016); Lebanon (Talhouk 1961; Mackauer \& Starý 1967; Starý 1974, 1976; Abou-Fakhr 1982; Hussein \& Kawar 1984; Tremblay et al. 1985); Libya (Starý 1976); Morocco (Starý \& Sekkat 1987); Syria (Starý 1974, 1976); Tunisia (Boukhris-Bouhachem 2011; Ayadi et al. 2017); Turkey (Uysal et al. 2004; Eastern part - Karaat \& Goven 1986; Ölmez \& Ulusoy 2003; Central part - Düzgüneş et al. 1982; Zeren \& Düzgüneş 1983; Aslan et al. 2004; Satar et al. 2014; Western part - Erkin 1983; Özder \& Toros 1999; Aslan \& Karaca 2005; Žikić et al. 2009; Yoldaş et al. 2011; Akar \& Erdoğan 2017); UAE (Starý et al. 2013); Yemen (Starý et al. 2013).

\section{General distribution}

Cosmopolitan. 


\section{Host records}

Acyrthosiphon pisum (Harris), Aphis fabae Scopoli, Aphis pomi De Geer (Düzgüneş et al. 1982; Uysal et al. 2004); Brachycaudus cardui (L.) (Starý et al. 2000); Brachycaudus helichrysi (Kaltenbach) (Rakhshani 2012); Corylobium avellanae (Schrank) (Düzgüneş et al. 1982; Uysal et al. 2004); Diuraphis noxia (Kurdjumov) (Pike et al. 1991; Uysal et al. 2004); Dysaphis crataegi (Kaltenbach) (Düzgüneş et al. 1982; Starý et al. 2000; Uysal et al. 2004); Dysaphis devecta (Walker), Dysaphis plantaginea (Passerini) (Düzgüneş et al. 1982; Uysal et al. 2004); Dysaphis pyri (Boyer de Fonscolombe) (Rakhshani 2012); Dysaphis sp. (Starý 1979); Hyalopterus pruni (Geoffroy) (Düzgüneş et al. 1982; Hussein \& Kawar 1984; Uysal et al. 2004); Macrosiphum sp. (Starý 1976; Uysal et al. 2004); Myzus cerasi (F.) (Mokhtari et al. 2000; Starý et al. 2000); Rhopalosiphum oxyacanthae (Schrank) (Düzgüneş et al. 1982 - as Rhopalosiphum insertum (Walker); Uysal et al. 2004), Rhopalosiphum maidis (Fitch) (Düzgüneş et al. 1982; Uysal et al. 2004); Rhopalosiphum padi (L.) (Rakhshani et al. 2008b; Barahoei et al. 2013); Schizaphis graminum (Rondani) (Rakhshani et al. 2008b; Barahoei et al. 2014); Sitobion avenae (Fabricius) (Elmali \& Toros 1994; DarvishMojeni \& Bayat-Asadi 1995; Starý et al. 2000; Uysal et al. 2004; Rakhshani et al. 2008b; Nazari et al. 2012).

\section{Distribution in the Middle East and North Africa}

Egypt (Abdel-Rahman 2005; El-Ghiet et al. 2014); Iran (Starý 1979; Starý et al. 2000; Rakhshani 2012; Barahoei et al. 2013; Nazari et al. 2012); Iraq (Starý 1976); Lebanon (Hussein \& Kawar 1984); Turkey (Starý 1976; Uysal et al. 2004; Central part - Düzgüneş et al. 1982; Elmali \& Toros 1994; Western part - Erdoğan et al. 2008; Akar \& Erdoğan 2017).

\section{General distribution}

Australasian, Eastern and Western Palaearctic, Neotropical, Oceanic, Oriental.

Lipolexis gracilis Förster, 1862

Figs 36, 126, 229, 325, 426, 563

\section{Host records}

Aphis fabae Scopoli (Abou-Fakhr 1982; Tremblay et al. 1985; Abou-Fakhr \& Kawar 1998; Rakhshani et al. 2008d); Aphis gossypii Glover (Tremblay et al. 1985); Aphis pomi De Geer (Aslan \& Karaca 2005); Aphis salviae Walker (Barahoei et al. 2013; Alikhani et al. 2013); Aphis umbrella (Börner) (Abou-Fakhr 1982; Tremblay et al. 1985; Abou-Fakhr \& Kawar 1998); Aphis sp. (Starý 1976; Laamari et al. 2012); Brachycaudus amygdalinus (Schouteden) (Rakhshani et al. 2012b).

\section{Distribution in the Middle East and North Africa}

Algeria (Starý 1976; Laamari et al. 2012); Iran (Rakhshani et al. 2008c, 2012b; Barahoei et al. 2013; Alikhani et al. 2013); Lebanon (Abou-Fakhr 1982; Tremblay et al. 1985; Abou-Fakhr \& Kawar 1998); Turkey (Western part - Aslan \& Karaca 2005; Central part - Rakhshani et al. 2008d; Tomanović et al. 2008).

\section{General distribution}

Eastern and Western Palaearctic, Oriental. 
Lysiphlebus cardui (Marshall, 1896)

Figs 37, 127, 230, 326, 427, 504, 564

\section{Host records}

Aphis fabae Scopoli (Alikhani et al. 2013); Aphis gossypii Glover (?) (Laamari et al. 2012).

\section{Distribution in the Middle East and North Africa}

Algeria (Laamari et al. 2012); Iran (Alikhani et al. 2013).

\section{General distribution}

Eastern (Iran) and Western Palaearctic.

Lysiphlebus confusus Tremblay \& Eady, 1978

Figs 38, 128, 231, 327, 428, 505, 565

Lysiphlebus marismortui Mescheloff \& Rosen, 1990a, Israel Journal of Entomology, 24: 35-50. syn. nov.

\section{Host records}

Acyrthosiphon sp. (?) (Starý et al. 2000); Aphis affinis del Guercio (Taheri \& Rakhshani 2013); Aphis aurantii Boyer de Fonscolombe (Rosen 1967, 1969; Avidov \& Harpaz 1969; Starý 1976; Tremblay et al. 1985; Ben Halima et al. 1994; Starý 1976; Aghajanzadeh et al. 1995; Starý et al. 2000; Uysal et al. 2004; Ben Halima \& Ben Hamouda 2005); Aphis craccivora Koch (Al-Azawi 1970; Starý \& Kaddou 1971; Starý 1976, 1979; Abou-Fakhr 1982; Tremblay et al. 1985; Mescheloff \& Rosen 1990a; Abou-Fakhr \& Kawar 1998; Starý et al. 2000; Uysal et al. 2004; Güz \& Kilinçer 2005; Talebi et al. 2009; Nazari et al. 2012; Taheri \& Rakhshani 2013; Barahoei et al. 2013; Satar et al. 2014; Chaouche \& Laamari 2015; Rakhshani et al. 2005a, 2006a); Aphis euphorbiae Kaltenbach (Güz \& Kilinçer 2005); Aphis fabae Scopoli (Starý 1976; Al-Azawi 1970; Starý \& Kaddou 1971; Abou-Fakhr 1982; Hussein \& Kawar 1984; Tremblay et al. 1985; Mescheloff \& Rosen 1990a; Ben Halima \& Ben Hamouda 1993, 2005; Ben Halima et al. 1994 - as Aphis citricola van der Goot; Abou-Fakhr \& Kawar 1998; Ölmez \& Ulusoy 2003; Aslan et al. 2004; Ben Halima \& Ben Hamouda 2005 - as Aphis citricola van der Goot; Uysal et al. 2004; Talebi et al. 2009; Laamari et al. 2011, 2012; Nazari et al. 2012); Aphis farinosa Gmelin (Starý et al. 2000; Uysal et al. 2004; Rakhshani et al. 2007b; Talebi et al. 2009; Nazari et al. 2012); Aphis gossypii Glover (Starý 1976; Al-Azawi 1970; Starý \& Kaddou 1971; Mescheloff \& Rosen 1990a; Ben Halima \& Ben Hamouda 1993, 2005; Ben Halima et al. 1994; Ölmez \& Ulusoy 2003; Uysal et al. 2004; Laamari et al. 2011; Nazari et al. 2012; Satar et al. 2014); Aphis hederae Kaltenbach (Abou-Fakhr 1982; Tremblay et al. 1985; Mescheloff \& Rosen 1990a; Abou-Fakhr \& Kawar 1998); Aphis idaei van der Goot (Barahoei et al. 2013; Taheri \& Rakhshani 2013); Aphis intybi Koch (Barahoei et al. 2013; Taheri \& Rakhshani 2013); Aphis nasturtii Kaltenbach (Al-Azawi 1970; Starý 1976; Laamari et al. 2012); Aphis nasturtii Kaltenbach (Mescheloff \& Rosen 1990a - as Aphis zizyphi Theobald); Aphis nerii Boyer de Fonscolombe (Al-Azawi 1970; Starý 1976; Ben Halima et al. 1994; Ben Halima \& Ben Hamouda 2005); Aphis potentillae Nevsky (Laamari et al. 2011, 2012); Aphis punicae Passerini (Aslan et al. 2004; Uysal et al. 2004); Aphis ruborum (Börner) (Starý 1976; Mescheloff \& Rosen 1990a; Aslan et al. 2004; Uysal et al. 2004); Aphis solanella Theobald (Abou-Fakhr 1982; Tremblay et al. 1985; Abou-Fakhr \& Kawar 1998); Aphis umbrella (Börner) (Abou-Fakhr 1982; Tremblay et al. 1985; Mescheloff \& Rosen 1990a; Abou-Fakhr \& Kawar 1998); Aphis urticata Gmelin (Güz \& Kilinçer 2005); Aphis vallei Hille Ris Lambers \& Stroyan (Starý 1976; Güz \& Kilinçer 2005); Aphis verbasci Schrank (Mescheloff \& Rosen 1990a; Starý et al. 2000; Talebi et al. 2009; Rakhshani 
et al. 2012b; Barahoei et al. 2013); Aulacorthum sp. (Starý 1979); Brachycaudus amygdalinus (Schouteden) (Mescheloff \& Rosen 1990a); Brachycaudus cardui (L.) (Abou-Fakhr 1982; Tremblay et al. 1985; Abou-Fakhr \& Kawar 1998; Starý et al. 2000; Uysal et al. 2004); Brachycaudus helichrysi (Kaltenbach) (Aslan et al. 2004; Uysal et al. 2004; Nazari et al. 2012; Satar et al. 2014); Brachyunguis skafi Remaudière \& Talhouk (Starý et al. 2000); Dysaphis crataegi (Kaltenbach); Dysaphis foeniculus (Theobald) (Güz \& Kilinçer 2005); Ephedraphis ephedrae (Nevsky) (Starý 1979; Starý et al. 2000); Hyadaphis foeniculi (Passerini) (Tremblay et al. 1985); Hyperomyzus lactucae (L.) (?) (Barahoei et al. 2013); Macrosiphoniella tapuskae (Hottes \& Frison) (?) (Starý 1979; Starý et al. 2000; Rakhshani et al. 2011); Melanaphis sp. (Mescheloff \& Rosen 1990a); Myzus persicae (Sulzer) (Ben Halima et al. 1994; Abou-Fakhr \& Kawar 1998); Protaphis sp. (Starý 1979); Uroleucon sp. (?) (Barahoei et al. 2012, 2013).

\section{Distribution in the Middle East and North Africa}

Algeria (Starý 1976; Laamari et al. 2011, 2012; Chaouche \& Laamari 2015); Iran (Starý 1979; Aghajanzadeh et al. 1995; Starý et al. 2000; Rakhshani et al. 2005a; Rakhshani et al. 2007b; Barahoei et al. 2012, 2013; Nazari et al. 2012; Rakhshani et al. 2012b; Taheri \& Rakhshani 2013); Egypt (Mackauer \& Starý 1967; Starý 1976); Iraq (Starý 1969a, 1976, 1979; Al-Azawi 1970; Starý \& Kaddou 1971); Israel (Rosen 1967, 1969; Avidov \& Harpaz 1969; Starý 1976; Mescheloff \& Rosen 1990a); Lebanon (Abou-Fakhr 1982; Hussein \& Kawar 1984; Tremblay et al. 1985; Abou-Fakhr \& Kawar 1998); Tunisia (Ben Halima \& Ben Hamouda 1993, 2005; Ben Halima et al. 1994); Turkey (Starý 1976; Uysal et al. 2004; Eastern part - Ölmez \& Ulusoy 2003; Aslan et al. 2004; Güz \& Kilinçer 2005; Satar et al. 2014; Akar \& Erdoğan 2017); UAE (Starý et al. 2013).

\section{General distribution}

Eastern and Western Palaearctic, Oriental.

\section{Remarks}

Mescheloff \& Rosen (1990a) described Lysiphlebus marismortui from Israel. Based on the original description of this species, which is very closely related to Lysiphlebus confusus, and the fact that Lysiphlebus confusus exhibits major morphological variability (Tomanović et al. 2018), we believe that Lysiphlebus marismortui falls as a synonym of Lysiphlebus confusus.

Lysiphlebus desertorum Starý, 1965

Figs 39, 129, 232, 328, 429, 506, 566

\section{Host records}

Aphis craccivora Koch (Barahoei et al. 2013); Aphis sp. (Rakhshani et al. 2012b); Protaphis terricola (Rondani) (Barahoei et al. 2013); Protaphis sp. (Talebi et al. 2009; Nazari et al. 2012); Xerobion cinae (Nevsky) (Starý 1979; Starý et al. 2000; Barahoei et al. 2013).

\section{Distribution in the Middle East and North Africa}

Iran (Starý 1979; Starý et al. 2000; Talebi et al. 2009; Nazari et al. 2012; Rakhshani et al. 2012b; Barahoei et al. 2013).

\section{General distribution}

Eastern (Iran, Uzbekistan) and Western (Spain) Palaearctic. 
Lysiphlebus fabarum (Marshall, 1896)

Figs 40, 130, 233, 329, 430, 507, 567

\section{Material examined}

SAUDI ARABIA • 11 우, 3 § ô; Abha; May 2013; on Lablab purpureus; Z. Ahmad leg. (DPPZ).

\section{Host records}

Acyrthosiphon ilka Mordvilko (?) (Starý 1979; Starý et al. 2000); Acyrthosiphon gossypii Mordvilko (?) (Barahoei et al. 2013; Rakhshani et al. 2013); Acyrthosiphon lactucae (Passerini) (?) (Starý et al. 2000); Acyrthosiphon pisum (Harris) (?) (Zeren \& Düzgüneş 1983; Uysal et al. 2004); Aphis acetosae L. (Starý et al. 2000; Barahoei et al. 2013; Rakhshani et al. 2013); Aphis affinis Del Guercio (Starý \& Kaddou 1971; Starý 1976; Starý \& Sekkat 1987; Starý et al. 2000; Uysal et al. 2004; Talebi et al. 2009; Rakhshani et al. 2012b; Nazari et al. 2012; Tomanović et al. 2012; Barahoei et al. 2013; Rakhshani et al. 2013; Taheri \& Rakhshani 2013); Aphis althaeae (Nevsky) (Starý 1979; Starý et al. 2000; Rakhshani et al. 2012b); Aphis astragali Ossiannilsson (Laamari et al. 2011, 2012); Aphis aurantii Boyer de Fonscolombe (Rosen 1967, 1969; Avidov \& Harpaz 1969; Starý 1976; Hussein \& Kawar 1984; Tremblay et al. 1985; Yumruktepe \& Uygun 1994; Aghajanzadeh et al. 1995; Starý et al. 2000; Uysal et al. 2004; Boukhris-Bouhachem 2011; Rakhshani et al. 2013); Aphis brunnea Ferrari (Chaouche \& Laamari 2015); Aphis chloris Koch (Mescheloff \& Rosen 1990a); Aphis craccivora Koch (Starý 1976, 1979; Starý \& Kaddou 1971; Monajemi \& Esmaili 1981; Düzgüneş et al. 1982; Zeren \& Düzgüneş 1983; Abou-Fakhr 1982; Tremblay et al. 1985; Starý \& Sekkat 1987; Mescheloff \& Rosen 1990a; Abou-Fakhr \& Kawar 1998; Starý et al. 2000; Ölmez \& Ulusoy 2003; Aslan et al. 2004; Uysal et al. 2004; Güz \& Kilinçer 2005; Rakhshani et al. 2005a, 2006a; AbdelSamad \& Ahmed 2009; Talebi et al. 2009; Mossadegh et al. 2011; Barahoei et al. 2011, 2012, 2013; Laamari et al. 2011, 2012; Nazari et al. 2012; Rakhshani et al. 2012b, 2013; Taheri \& Rakhshani 2013; Satar et al. 2014); Aphis davletshinae Hillie Ris Lambers (Ölmez \& Ulusoy 2003; Uysal et al. 2004; Rakhshani et al. 2013); Aphis epilobiaria Theobald (Mescheloff \& Rosen 1990a); Aphis epilobii Kaltenbach (Nazari et al. 2012; Rakhshani et al. 2013); Aphis euphorbiae Kaltenbach (Rakhshani et al. 2013); Aphis euphorbicola Rezwani \& Lampel (Rakhshani et al. 2012b, 2013; Barahoei et al. 2013); Aphis fabae Scopoli (Davatchi \& Shojai 1968; Starý 1976; Al-Azawi 1970; Starý \& Kaddou 1971; Abou-Fakhr 1982; Düzgüneş et al. 1982; Zeren \& Düzgüneş 1983; Tremblay et al. 1985; Starý \& Sekkat 1987; Mescheloff \& Rosen 1990a; Yumruktepe \& Uygun 1994; AbouFakhr \& Kawar 1998; Starý et al. 2000; Ölmez \& Ulusoy 2003; Aslan et al. 2004; Uysal et al. 2004; Güz \& Kilinçer 2005; Talebi et al. 2009; Laamari et al. 2011, 2012; Rakhshani et al. 2012b, 2013; Nazari et al. 2012; Taheri \& Rakhshani 2013; Barahoei et al. 2011, 2012, 2013; Chaouche \& Laamari 2015); Aphis fabae cirsiiacanthoidis Scopoli (Aslan et al. 2004; Uysal et al. 2004; Rakhshani et al. 2013); Aphis galiiscabri Schrank (Güz \& Kilinçer 2005); Aphis gerardianae Mordvilko (Rakhshani et al. 2012b, 2013); Aphis gossypii Glover (Avidov \& Harpaz 1969; Al-Azawi 1970; Starý 1976, 1979; Abou-Fakhr 1982; Düzgüneş et al. 1982; Zeren \& Düzgüneş 1983; Tremblay et al. 1985; Starý \& Sekkat 1987; Mescheloff \& Rosen 1990a; Yumruktepe \& Uygun 1994; Abou-Fakhr \& Kawar 1998; Starý et al. 2000; Ölmez \& Ulusoy 2003; Uysal et al. 2004; Talebi et al. 2009; Laamari et al. 2011, 2012; Boukhris-Bouhachem 2011; Mossadegh et al. 2011; Rakhshani et al. 2012b, 2013; Nazari et al. 2012; Barahoei et al. 2011, 2012, 2013; Taheri \& Rakhshani 2013; Satar et al. 2014); Aphis hederae Kaltenbach (Abou-Fakhr 1982; Tremblay et al. 1985; Mescheloff \& Rosen 1990a; Abou-Fakhr \& Kawar 1998); Aphis idaei van der Goot (Talebi et al. 2009; Taheri \& Rakhshani 2013; Rakhshani et al. 2013; Barahoei et al. 2013); Aphis intybi Koch (Düzgüneş et al. 1982; Starý et al. 2000; Aslan et al. 2004; Uysal et al. 2004; Güz \& Kilinçer 2005; Talebi et al. 2009; Rakhshani et al. 2012b; Taheri \& Rakhshani 2013); Aphis nasturtii Kaltenbach (Starý 1976, 1979; Starý et al. 2000; Nazari et al. 2012; Laamari et al. 2012; Rakhshani et al. 2013; Barahoei et al. 2013); Aphis nerii 
RAKHSHANI E. et al., Aphidiinae of the Middle East and North Africa

Boyer de Fonscolombe (Starý et al. 2000; Aslan et al. 2004; Uysal et al. 2004; Talebi et al. 2009; Barahoei et al. 2013; Rakhshani et al. 2013); Aphis origani Passerini, Aphis plantaginis Goeze (Barahoei et al. 2013; Rakhshani et al. 2013); Aphis pomi De Geer (Düzgüneş et al. 1982; Radjabi 1989; Starý et al. 2000; Uysal et al. 2004; Rakhshani 2012); Aphis punicae (Passerini) (Starý 1976; Mescheloff \& Rosen 1990a; Talebi et al. 2009; Laamari et al. 2012; Nazari et al. 2012; Barahoei et al. 2013; Rakhshani et al. 2013; Taheri \& Rakhshani 2013); Aphis ruborum (Börner) (Starý \& Kaddou 1971; Starý 1976, 1979; Düzgüneş et al. 1982; Starý et al. 2000; Uysal et al. 2004; Güz \& Kilinçer 2005; Rakhshani et al. 2013); Aphis rumicis L. (Starý et al. 2000; Güz \& Kilinçer 2005; Talebi et al. 2009; Nazari et al. 2012; Rakhshani et al. 2013); Aphis salviae Walker (Starý 1979; Starý et al. 2000; Güz \& Kilinçer 2005); Aphis solanella Theobald (Starý \& Kaddou 1971; Starý 1976; Düzgüneş et al. 1982; Zeren \& Düzgüneş 1983; Uysal et al. 2004; Güz \& Kilinçer 2005; Nazari et al. 2012; Rakhshani et al. 2013 Talebi et al. 2009 - as Aphis evonymi F.; Barahoei et al. 2013; Rakhshani et al. 2013 - as Aphis evonymi F.); Aphis spiraecola Patch (Starý et al. 2000; Laamari et al. 2012; Rakhshani 2012; Rakhshani et al. 2013); Aphis taraxacicola (Börner) (Starý 1979; Starý et al. 2000); Aphis tirucallis Hille Ris Lambers (Ölmez \& Ulusoy 2003; Uysal et al. 2004); Aphis umbrella (Börner) (Abou-Fakhr 1982; Tremblay et al. 1985; Mescheloff \& Rosen 1990a; Abou-Fakhr \& Kawar 1998; Starý et al. 2000; Talebi et al. 2009; Nazari et al. 2012; Barahoei et al. 2013; Rakhshani et al. 2013); Aphis urticata Gmelin (Starý et al. 2000; Güz \& Kilinçer 2005; Rakhshani et al. 2013), Aphis verbasci Schrank (Güz \& Kilinçer 2005); Aphis sp. (Starý \& Kaddou 1971; Starý 1976, 1979; Starý et al. 2000; Güz \& Kilinçer 2005; Barahoei et al. 2013; Laamari et al. 2012; Nazari et al. 2012); Brachycaudus amygdalinus (Schouteden) (Nazari et al. 2012; Rakhshani 2012); Brachycaudus cardui (L.) (Düzgüneş et al. 1982; Elmali \& Toros 1994; Starý et al. 2000; Aslan et al. 2004; Uysal et al. 2004; Talebi et al. 2009; Laamari et al. 2011; Rakhshani et al. 2012b; 2013; Nazari et al. 2012; Barahoei et al. 2013; Taheri \& Rakhshani 2013); Brachycaudus helichrysi (Kaltenbach) (Starý 1976; Starý et al. 2000; Aslan et al. 2004; Uysal et al. 2004; Jafari et al. 2011; Laamari et al. 2011, 2012; Jafari \& Modarres Awal 2012; Nazari et al. 2012; Rakhshani 2012; Barahoei et al. 2013; Rakhshani et al. 2013; Taheri \& Rakhshani 2013); Brachycaudus persicae (Passerini) (Rakhshani 2012); Brachycaudus tragopogonis (Kaltenbach) (Düzgüneş et al. 1982; Uysal et al. 2004; Barahoei et al. 2012, 2013; Rakhshani et al. 2012b, 2013); Brachycaudus tragopogonis setosus (Hille Ris Lambers) (Starý et al. 2000); Brachyunguis harmalae Das (Talebi et al. 2009; Rakhshani et al. 2012b, 2013); Brachyunguis zygophylli (Nevsky) (Barahoei et al. 2011, 2012, 2013; Rakhshani et al. 2013); Cavariella aegopodii (Scopoli) (Abou-Fakhr 1982; Tremblay et al. 1985; Uysal et al. 2004); Capitophorus elaeagni (Del Guercio) (Düzgüneş et al. 1982; Uysal et al. 2004); Coloradoa sp. (Starý 1979; Starý et al. 2000); Dysaphis lappae Koch (Starý 1979); Dysaphis plantaginea (Passerini) (Düzgüneş et al. 1982; Starý et al. 2000; Aslan et al. 2004; Uysal et al. 2004; Rakhshani 2012; Barahoei et al. 2013; Rakhshani et al. 2013); Dysaphis radicola (Mordvilko) (Talebi et al. 2009; Rakhshani et al. 2013); Dysaphis rumecicola emicis (Mimeur) (Mescheloff \& Rosen 1990a); Hayhurstia atriplicis (L.) (Nazari et al. 2012; Rakhshani et al. 2013); Hyadaphis coriandri (Das) (Talebi et al. 2009; Rakhshani et al. 2013); Hyadaphis foeniculi (Passerini) (Tremblay et al. 1985); Hyalopterus amygdali (Blanchard) (?) (Rakhshani 2012); Hyalopterus pruni (Geoffroy) (?) (Starý 1976; Erkin 1983; Uysal et al. 2004; Jafari et al. 2011; Jafari \& Modarres Awal 2012; Laamari et al. 2012); Lipaphis erysimi (Kaltenbach) (Talebi et al. 2009; Rakhshani et al. 2013), Lipaphis fritzmuelleri Börner (Talebi et al. 2009); Lipaphis lepidii (Nevsky) (Starý 1979; Starý et al. 2000; Talebi et al. 2009; Rakhshani et al. 2013); Macrosiphoniella papillata Holman (?) (Barahoei et al. 2013; Rakhshani et al. 2013); Macrosiphoniella sanborni (Gillette) (?) (Starý et al. 2000); Macrosiphum rosae (L.) (?) (Taheri \& Rakhshani 2013); Melanaphis sacchari (Zehntner) (Barahoei et al. 2011; Rakhshani et al. 2013); Melanaphis sp. (Barahoei et al. 2012, 2013); Metopolophium dirhodum (Walker) (?) (Barahoei et al. 2013; Rakhshani et al. 2013); Myzus beybienkoi (Narzikulov) (Talebi et al. 2009; Rakhshani et al. 2013); Myzus persicae (Sulzer) (Karaat \& Goven 1986; Mescheloff \& Rosen 1990a; Ölmez \& Ulusoy 2003; Uysal et al. 2004; 
Güz \& Kilinçer 2005; Talebi et al. 2009; Boukhris-Bouhachem 2011; Laamari et al. 2011, 2012; Barahoei et al. 2013; Rakhshani et al. 2013); Phorodon humuli (Schrank) (Mokhtari et al. 2000; Starý et al. 2000); Protaphis alexandrae (Nevsky) (Rakhshani et al. 2012b, 2013); Protaphis anthemidis (Börner) (Talebi et al. 2009; Nazari et al. 2012; Rakhshani et al. 2013); Protaphis elongata (Nevsky) (Talebi et al. 2009; Rakhshani et al. 2013); Protaphis terricola (Rondani) (Güz \& Kilinçer 2005; Barahoei et al. 2013; Rakhshani et al. 2013); Protaphis sp. (Starý 1976, 1979; Mescheloff \& Rosen 1990a; Barahoei et al. 2013); Rhopalosiphum maidis (Fitch) (Starý 1976; Düzgüneş et al. 1982; Özder \& Toros 1999; Uysal et al. 2004); Rhopalosiphum nymphaeae (L.) (Mokhtari et al. 2000; Starý et al. 2000); Rhopalosiphum padi (L.) (Rakhshani et al. 2008b, 2013; Nazari et al. 2012; Taheri \& Rakhshani 2013; Gadallah et al. 2017); Saltusaphis scirpus Theobald (Starý et al. 2000; Tomanović et al. 2012); Saltusaphis sp. (Starý 1979); Sitobion avenae (Fabricius) (?) (Elmali \& Toros 1994; Özder \& Toros 1999; Uysal et al. 2004); Uroleucon compositae (Theobald) (?) (Laamari et al. 2011); Uroleucon jaceae (L.) (?) (Aslan et al. 2004; Uysal et al. 2004; Güz \& Kilinçer 2005).

\section{Distribution in the Middle East and North Africa}

Algeria (Starý 1976; Laamari et al. 2011, 2012; Chaouche \& Laamari 2015); Egypt (Mackauer \& Starý 1967; Starý 1976; Abdel-Samad \& Ahmed 2009; Gadallah et al. 2017); Iran (Starý 1979; Aghajanzadeh et al. 1995; Starý et al. 2000; Rakhshani et al. 2005a, 2006a, 2012b, 2013; Mossadegh et al. 2011; Nazari et al. 2012; Rakhshani 2012; Barahoei et al. 2011, 2012, 2013; Taheri \& Rakhshani 2013); Iraq (Starý 1969a, 1976; Al-Azawi 1970; Starý \& Kaddou 1971); Israel (Rosen 1967, 1969; Avidov \& Harpaz 1969; Starý 1976; Mescheloff \& Rosen 1990a); Lebanon (Mackauer \& Starý 1967; Starý 1976; Abou-Fakhr 1982; Hussein \& Kawar 1984; Tremblay et al. 1985; Abou-Fakhr \& Kawar 1998); Morocco (Mimeur 1934; Starý 1976; Starý \& Sekkat 1987); Syria (Starý 1976); Tunisia (BoukhrisBouhachem 2011); Turkey (Starý 1976; Eastern part - Karaat \& Goven 1986; Ölmez \& Ulusoy 2003; Aslan et al. 2004; Uysal et al. 2004; Güz \& Kilinçer 2005; Central part - Satar et al. 2014; Western part - Erkin 1983; Erdoğan et al. 2008; Akar \& Erdoğan 2017); UAE (Starý et al. 2013).

\section{General distribution}

Australasian, Eastern and Western Palaearctic, Oceanic, Oriental.

\section{Remarks}

Specimens reared from Brachycaudus tragopogonis (Kaltenbach) are most probably refer to Lysiphlebus volkli Tomanović \& Kavallieratos, 2018.

\section{Lysiphlebus fritzmuelleri Mackauer, 1960}

Figs 41, 131, 234, 330, 431, 508, 568

\section{Host records}

Lacking host data (Akar \& Erdoğan 2017).

\section{Distribution in the Middle East and North Africa}

Turkey (Western part - Akar \& Erdoğan 2017).

\section{General distribution}

Australasian, Eastern Palaearctic (Kazakhstan), Western Palaearctic (Eastern and central Europe), Oceanic, Oriental. 
RAKHSHANI E. et al., Aphidiinae of the Middle East and North Africa

Lysiphlebus testaceipes (Cresson, 1880)

Figs 42, 132, 235, 331, 432, 509, 569

\section{Host records}

Acyrthosiphon gossypii Mordvilko (Chaouche \& Laamari 2015); Aphis astragali Ossiannilsson (Laamari et al. 2012); Aphis aurantii Boyer de Fonscolombe (Boukhris-Bouhachem 2011; Yoldaş et al. 2011); Aphis brunnea Ferrari (Chaouche \& Laamari 2015); Aphis craccivora Koch (Rakhshani et al. 2005a; Yoldaş et al. 2011; Laamari et al. 2011, 2012; Chaouche \& Laamari 2015); Aphis euphorbiae Kaltenbach (Laamari et al. 2011, 2012); Aphis fabae Scopoli (Laamari et al. 2011, 2012); Aphis gossypii Glover (Ben Halima 2011; Boukhris-Bouhachem 2011; Yoldaş et al. 2011; Laamari et al. 2011, 2012; Chaouche \& Laamari 2015; Ayadi et al. 2017); Aphis illinoisensis Shimer (Havelka et al. 2011; Laamari et al. 2011, 2012); Aphis nerii Boyer de Fonscolombe (Laamari et al. 2011, 2012; Chaouche \& Laamari 2015; Ayadi et al. 2017); Aphis pomi De Geer (Laamari et al. 2011, 2012; Mdellel \& Ben Halima 2015), Aphis potentillae Nevsky, Aphis punicae (Passerini) and Aphis solanella Theobald (Ayadi et al. 2017); Aphis spiraecola Patch (Labdaoui \& Guenaoui 2018); Brachycaudus cardui (L.) and Brachycaudus helichrysi (Kaltenbach) (Laamari et al. 2011, 2012); Diuraphis noxia (Kurdjumov) (Laamari et al. 2016); Brevicoryne brassicae (L.) (Uysal et al. 2004); Dysaphis foeniculus (Theobald) (Chaouche \& Laamari 2015); Dysaphis plantaginea (Passerini); Dysaphis pyri (Boyer de Fonscolombe); Dysaphis tulipae (Boyer de Fonscolombe); Hyalopterus pruni (Geoffroy) (Laamari et al. 2011, 2012); Myzus persicae (Sulzer) (Boukhris-Bouhachem 2011; Yoldaş et al. 2011; Laamari et al. 2011, 2012; Chaouche \& Laamari 2015); Macrosiphum euphorbiae (Thomas) (Laamari et al. 2011, 2012); Rhopalosiphum maidis (Fitch) (Boukhris-Bouhachem 2011; Yoldaş et al. 2011; Laamari et al. 2011, 2012; Ayadi et al. 2017); Uroleucon compositae (Theobald) (Laamari et al. 2012).

\section{Distribution in the Middle East and North Africa}

Algeria (Havelka et al. 2011; Laamari et al. 2011, 2012; Chaouche \& Laamari 2015; Laamari et al. 2016; Labdaoui \& Guenaoui 2018); Egypt (Laboratory population - Adly \& El-Gantiry 2014); Iran (Rakhshani et al. 2005a); Libya (Mitrović et al. 2013; Žikić et al. 2015); Tunisia (Ben Halima 2011; BoukhrisBouhachem 2011; Ayadi et al. 2017); Turkey (Uysal et al. 2004; Western part - Yoldaş et al. 2011).

\section{General distribution}

Cosmopolitan.

\section{Host records}

Lysiphlebus volkli Tomanović \& Kavallieratos, 2018

Aphis verbasci Schrank, Brachycaudus tragopogonis (Kaltenbach) (Tomanović et al. 2018).

\section{Distribution in the Middle East and North Africa}

Iran (Tomanović et al. 2018).

\section{General distribution}

Eastern Palaearctic (Iran), Western Palaearctic (Southeastern Europe).

Monoctonia pistaciaecola Starý, 1962

Figs 43, 133, 236, 332, 433, 570

\section{Host records}

Forda sp. (Starý \& Kaddou 1971; Starý 1976); Smynthurodes betae Westwood (Wool \& Burstein 1991); Lacking host data (Kargarian et al. 2016). 


\section{Distribution in the Middle East and North Africa}

Iran (Kargarian et al. 2016), Iraq (Starý \& Kaddou 1971; Starý 1976); Israel (Wool \& Burstein 1991).

\section{General distribution}

Eastern and Western Palaearctic.

Monoctonia vesicarii Tremblay, 1991

Figs 44, 134, 237, 333, 434, 571

\section{Host records}

Pemphigus spyrothecae Passerini (Ghafouri Moghaddam et al. 2012; Rakhshani et al. 2015).

\section{Distribution in the Middle East and North Africa}

Iran (Ghafouri Moghaddam et al. 2012; Rakhshani et al. 2015).

\section{General distribution}

Eastern (Iran) and Western Palaearctic.

Monoctonus crepidis (Haliday, 1834)

Figs 135, 238, 334, 435, 572

\section{Host records}

Lacking host data (Tomanović et al. 2008).

\section{Distribution in the Middle East and North Africa}

Turkey (Central part - Tomanović et al. 2008).

\section{General distribution}

Western Palaearctic, Nearctic, Oriental.

\section{Monoctonus mali van Achterberg, 1989}

Figs 136, 239, 335, 436, 573

\section{Host records}

Ovatus insitus (Walker) (Ölmez \& Ulusoy 2003; Uysal et al. 2004).

\section{Distribution in the Middle East and North Africa}

Iran (Mosavi et al. 2012); Turkey (Eastern part - Ölmez \& Ulusoy 2003; Uysal et al. 2004).

\section{General distribution}

Eastern (Iran) and Western Palaearctic.

Pauesia abietis (Marshall, 1896)

Figs 137, 240, 336, 437, 574

\section{Host records}

Cinara pinimaritimae (Dufour) (Uysal et al. 2004 - as Cinara maritimae (Dufour)); Cinara pilicornis (Hartig) (Schimitschek, 1944; Starý 1976 - as Cinara pinicola (Kaltenbach)). 


\section{Distribution in the Middle East and North Africa}

Turkey (Schimitschek 1944; Starý 1976; Uysal et al. 2004).

\section{General distribution}

Afrotropical, Eastern and Western Palaearctic, Oriental.

\section{Pauesia anatolica Michelena, Assael \& Mendel, 2005}

Figs 138, 241, 337, 438, 575

\section{Host records}

Cinara cedri Mimeur (Michelena et al. 2005).

\section{Distribution in the Middle East and North Africa}

Israel (imported); Turkey (Western part - Michelena et al. 2005).

\section{General distribution}

Western Palaearctic.

Pauesia antennata (Mukerji, 1950)

Figs 45, 139, 242, 338, 439, 576

\section{Host records}

Pterochloroides persicae (Cholodkovsky) (Mackauer \& Starý 1967; Starý \& Kaddou 1971; Starý 1976; Starý et al. 2000; Rakhshani et al. 2005b, 2012b; Talebi et al. 2009; Nazari et al. 2012; Rakhshani 2012; Barahoei et al. 2013).

\section{Distribution in the Middle East and North Africa}

Iran (Mackauer \& Starý 1967; Rakhshani et al. 2005b, 2012b; Nazari et al. 2012; Rakhshani 2012; Barahoei et al. 2013); Iraq (Starý \& Kaddou 1971; Starý 1976); Tunisia (Mdellel et al. 2015); Yemen (Cross \& Poswal 1996).

\section{General distribution}

Eastern and Western Palaearctic, Oriental.

\section{Pauesia cedrobii Starý \& Leclant, 1977}

Figs 140, 339, 440, 577

\section{Host records}

Cinara (Cedrobium) laportei Remaudière (Starý \& Leclant 1977).

\section{Distribution in the Middle East and North Africa}

Morocco (Starý \& Leclant 1977; Fabre \& Rabasse 1987).

\section{General distribution}

Western Palaearctic. 
Pauesia hazratbalensis Bhagat, 1981

Figs 46, 141, 243, 340, 441, 578

\section{Host records}

Cinara (Cupressobium) tujafilina (del Guercio) (Starý et al. 2005; Rakhshani et al. 2012b, 2017); Cinara pinihabitans (Mordvilko) (Heidari Latibari et al. 2019).

\section{Distribution in the Middle East and North Africa}

Iran (Starý et al. 2005; Rakhshani et al. 2012b; 2017).

\section{General distribution}

Eastern Palaearctic, Oriental.

Pauesia picta (Haliday, 1834)

Figs 142, 244, 341, 442, 579

\section{Host records}

Cinara pini (L.) (Aslan et al. 2004; Uysal et al. 2004).

\section{Distribution in the Middle East and North Africa}

Turkey (Central part - Aslan et al. 2004; Uysal et al. 2004).

\section{General distribution}

Western Palaearctic, Oriental.

\section{Pauesia pini (Haliday, 1834)}

Figs 143, 342, 443, 580

\section{Host records}

Cinara palaestinensis Hille Ris Lambers (Bodenheimer \& Swirski 1957; Avidov \& Harpaz 1969; Starý 1976).

\section{Distribution in the Middle East and North Africa}

Israel (Bodenheimer \& Swirski 1957; Avidov \& Harpaz 1969; Starý 1976).

\section{General distribution}

Eastern and Western Palaearctic, Oriental.

Pauesia silana Tremblay, 1969

Figs 144, 245, 343, 444, 581

\section{Host records}

Cinara palaestinensis Hille Ris Lambers (Mescheloff \& Rosen 1990b); Cinara pini (L.) (Benhamacha et al. 2017).

\section{Distribution in the Middle East and North Africa}

Algeria (Benhamacha et al. 2017), Israel (Mescheloff \& Rosen 1990b). 


\section{General distribution}

Western Palaearctic.

Pauesia unilachni (Gahan, 1926)

Figs 145, 344, 445, 582

\section{Host records}

Cinara (Schizolachnus) pineti (Fabricius) (Düzgüneş et al. 1982; Uysal et al. 2004).

\section{Distribution in the Middle East and North Africa}

Turkey (Central part - Düzgüneş et al. 1982; Uysal et al. 2004).

\section{General distribution}

Afrotropical, Eastern and Western Palaearctic, Oriental.

\section{Host records}

Pauesia sp.

Cinara cedri Mimeur (Aslan et al. 2004; Uysal et al. 2004).

\section{Distribution in the Middle East and North Africa}

Saudi Arabia (Ahmad \& Bakr 2013); Turkey (Central part - Aslan et al. 2004; Uysal et al. 2004); Yemen (Starý et al. 2013).

Praon abjectum (Haliday, 1833)

Figs 47, 146, 246, 345, 446, 583

\section{Host records}

Aphis craccivora Koch, Aphis punicae Passerini, Aphis solanella Theobald, Aphis viticis Ferrari, Aphis sp. (Starý \& Kaddou 1971; Starý 1976); Brachycaudus cardui (L.) (Rakhshani et al. 2007a; Rakhshani 2012)

\section{Distribution in the Middle East and North Africa}

Iran (Rakhshani et al. 2007a; Rakhshani 2012); Iraq (Starý 1969a, 1976; Starý \& Kaddou 1971); Turkey (Western part - Tomanović et al. 2008; Akar \& Erdoğan 2017).

\section{General distribution}

Eastern and Western Palaearctic, Nearctic, Oriental.

Praon absinthii Bignell, 1894

Figs 48, 147, 247, 346, 447, 584

\section{Host records}

Macrosiphoniella absinthii (L.) (Starý 1979; Starý et al. 2000; Rakhshani et al. 2011); Macrosiphoniella oblonga (Mordvilko) (Rakhshani et al. 2007a, 2011; Talebi et al. 2009); Macrosiphoniella sanborni (Gillette) (Rakhshani et al. 2007a, 2011).

\section{Distribution in the Middle East and North Africa}

Iran (Starý 1979; Starý et al. 2000; Rakhshani et al. 2007a; Talebi et al. 2009). 


\section{General distribution}

Eastern and Western Palaearctic, Oriental.

Praon athenaeum Kavallieratos \& Lykouressis, 2000

Figs 148, 248, 347, 448, 585

\section{Host records}

Lacking host data (Akar \& Erdoğan 2017)

Distribution in the Middle East and North Africa

Turkey (Western part - Akar \& Erdoğan 2017).

\section{General distribution}

Western Palaearctic (Europe).

Praon barbatum Mackauer, 1967

Figs 49, 149, 249, 348, 449, 586

\section{Material examined}

SAUDI ARABIA • 4 우 ; Abha; Jun. 2012; Acyrthosiphon pisum (Harris) on Trifolium alexandrinum; Z. Ahmad leg. (DPPZ).

\section{Host records}

Acyrthosiphon pisum (Harris) (González et al. 1978; Starý \& González 1978; Starý 1979; Tremblay et al. 1985; Starý et al. 2000; Rakhshani et al. 2007a; Nazari et al. 2012; Barahoei et al. 2013; Taheri \& Rakhshani 2013); Nearctaphis bakeri (Cowen) (Barahoei et al. 2013).

\section{Distribution in the Middle East and North Africa}

Iran (Starý 1979; Starý et al. 2000; Rakhshani et al. 2007a; Nazari et al. 2012; Barahoei et al. 2013; Taheri \& Rakhshani 2013); Lebanon (Tremblay et al. 1985); Morocco (Starý 1976); Saudi Arabia (new record).

\section{General distribution}

Eastern and Western Palaearctic, Nearctic (imported).

Praon bicolor Mackauer, 1959

Figs 50, 150, 250, 349, 450, 587

\section{Host records}

Lacking host data (Farahani et al. 2015).

\section{Distribution in the Middle East and North Africa}

Iran (Farahani et al. 2015).

\section{General distribution}

Eastern (Iran) and Western Palaearctic. 
Praon exsoletum (Nees, 1811)

Figs 51, 151, 251, 350, 451, 588

\section{Material examined}

SAUDI ARABIA • 2 우, 2 §ో; Abha; Jun. 2012; Therioaphis trifolii (Monell) on Trifolium alexandrinum; Z. Ahmad leg. (DPPZ).

\section{Host records}

Acyrthosiphon pisum (Harris) (?) (Monajemi \& Esmaili 1981; Starý et al. 2000), Therioaphis riehmi (Börner) (van den Bosch 1957; Starý et al. 2000); Therioaphis trifolii (Monell) (Harpaz 1955; van den Bosch 1957; Farahbakhsh 1961; Starý 1979; Avidov \& Harpaz 1969; Starý 1976; Monajemi \& Esmaili 1981; Starý \& Erdelen 1982; Tremblay et al. 1985; Rasulian 1989; Mescheloff \& Rosen 1988; Starý et al. 2000; Uysal et al. 2004; Rakhshani et al. 2006a, 2007a; Laamari et al. 2011, 2012; Rakhshani et al. 2012b; Nazari et al. 2012; Barahoei et al. 2013); Therioaphis sp. (Starý \& Kaddou 1971; Starý 1976).

\section{Distribution in the Middle East and North Africa}

Algeria (Laamari et al. 2011, 2012); Egypt (Starý 1976); Iran (Starý et al. 2000; Rakhshani et al. 2006a, 2007a, 2012b; Nazari et al. 2012; Barahoei et al. 2013); Iraq (Starý \& Kaddou 1971; Starý 1976); Israel (Harpaz 1955; Avidov \& Harpaz 1969; Starý 1976; Mescheloff \& Rosen 1988); Lebanon (van den Bosch 1957; Starý 1976; Tremblay et al. 1985); Saudi Arabia (Ahmad \& Bakr 2013); Turkey (Starý 1976; Eastern part - van den Bosch 1957; Uysal et al. 2004; Western part - Erdoğan et al. 2008; Akar \& Erdoğan 2017); Yemen (Starý \& Erdelen 1982; Starý et al. 2013).

\section{General distribution}

Afrotropical, Holarctic, Neotropical.

Praon flavinode (Haliday, 1833)

Figs 52, 152, 252, 351, 452, 589

\section{Host records}

Tinocallis nevskyi Remaudière, Quednau \& Heie (Barahoei et al. 2010, 2012, 2013) Tuberculatus (Tuberculoides) albosiphonatus Hille Ris Lambers (Starý \& Kaddou 1971; Starý 1976); Tuberculatus (Tuberculoides) moerickei Hille Ris Lambers (Starý 1976); Tuberculatus sp. (Starý 1969b, 1976; Starý \& Kaddou 1971).

\section{Distribution in the Middle East and North Africa}

Iran (Barahoei et al. 2010, 2012, 2013); Iraq (Starý 1969b, 1976; Starý \& Kaddou 1971); Turkey (Western part - Akar \& Erdoğan 2017).

\section{General distribution}

Eastern and Western Palaearctic, Oriental.

Praon gallicum Starý, 1971

Figs 53, 153, 253, 352, 453, 590

\section{Host records}

Rhopalosiphum maidis (Fitch) (El-Serafy 1999); Rhopalosiphum padi (L.) (Ibrahim 1990a, 1990b; ElSerafy 1999; Bagheri-Matin et al. 2010; Nazari et al. 2012); Schizaphis graminum (Rondani) (El-Serafy 1999); Sitobion avenae (Fabricius) (El-Serafy 1999). 


\section{Distribution in the Middle East and North Africa}

Egypt (Ibrahim 1990a, 1990b; El-Serafy 1999); Iran (Bagheri-Matin et al. 2010; Nazari et al. 2012).

\section{General distribution}

Eastern and Western Palaearctic, Nearctic (imported), Neotropical (imported).

Praon longicorne Marshall, 1896

Figs 54, 154, 254, 353, 454, 591

\section{Host records}

Lacking host data (Tomanović et al. 2008).

\section{Distribution in the Middle East and North Africa}

Turkey (Central and Eastern part - Tomanović et al. 2008).

\section{General distribution}

Eastern and Western Palaearctic, Oriental.

Praon necans Mackauer, 1959

Figs 55, 155, 255, 354, 455, 592

\section{Host records}

Aphis craccivora Koch (Nazari et al. 2012); Aphis gossypii Glover, Aphis nerii Boyer de Fonscolombe (Mossadegh et al. 2011); Aphis punicae Passerini, Macrosiphoniella sanborni (Gillette) (Nazari et al. 2012); Myzus persicae (Sulzer) (Mossadegh et al. 2011); Rhopalosiphum maidis (Fitch) (El-Heneidy \& Abdel-Samad 2001; El-Heneidy et al. 2001, 2002, 2003); Rhopalosiphum nymphaeae (L.) (Nazari et al. 2012; Mossadegh et al. 2011); Rhopalosiphum padi (L.) (Abdel-Rahman et al. 2000; El-Heneidy \& Abdel-Samad 2001; El-Heneidy et al. 2001, 2002, 2003; Abdel-Rahman 2005; Mossadegh et al. 2011; Nazari et al. 2012); Schizaphis graminum (Rondani) (Abdel-Rahman et al. 2000; El-Heneidy \& AbdelSamad 2001; El-Heneidy et al. 2001, 2002, 2003; Abdel-Rahman 2005); Sitobion avenae (Fabricius) (El-Heneidy et al. 2001, 2002, 2003; Mossadegh et al. 2011).

\section{Distribution in the Middle East and North Africa}

Egypt (Abdel-Rahman et al. 2000; El-Heneidy \& Abdel-Samad 2001; El-Heneidy et al. 2001, 2002, 2003; Abdel-Rahman 2005; El-Ghiet et al. 2014); Iran (Nazari et al. 2012; Mossadegh et al. 2011); Iraq (Starý 1976).

\section{General distribution}

Eastern and Western Palaearctic, Oriental.

Praon nonveilleri Tomanović \& Kavallieratos, 2003

Figs 156, 256, 355, 456, 593

\section{Host records}

Lacking host data (Akar \& Erdoğan 2017).

\section{Distribution in the Middle East and North Africa}

Turkey (Western part - Akar \& Erdoğan 2017). 


\section{General distribution}

Western Palaearctic (Europe).

Praon orpheusi Kavallieratos, Athanassiou \& Tomanović, 2003

Figs 56, 157, 257, 356, 457, 594

\section{Host records}

Hyperomyzus lactucae (L.) (Rakhshani et al. 2007a; Talebi et al. 2009).

\section{Distribution in the Middle East and North Africa}

Iran (Rakhshani et al. 2007a, Talebi et al. 2009).

\section{General distribution}

Eastern (Iran) and Western Palaearctic (Bulgaria, Greece).

Praon pubescens Starý, 1961

Figs 57, 158, 258, 357, 458, 595

\section{Host records}

Nasonovia ribisnigri (Mosley) (Nazari et al. 2012); Lacking host data (Tomanović et al. 2008).

\section{Distribution in the Middle East and North Africa}

Iran (Nazari et al. 2012); Turkey (Central part - Tomanović et al. 2008; Western part - Akar \& Erdoğan 2017).

\section{General distribution}

Eastern (Iran) and Western Palaearctic, Oriental.

Praon rosaecola Starý, 1961

Figs 58, 159, 259, 358, 459, 596

\section{Host records}

Macrosiphum rosae (L.) (Mescheloff \& Rosen 1988; Rakhshani et al. 2007a, 2012b; Talebi et al. 2009; Barahoei et al. 2010, 2012, 2013); Wahlgreniella nervata (Gillette) (Barahoei et al. 2013).

\section{Distribution in the Middle East and North Africa}

Iran (Rakhshani et al. 2007a; 2012b; Barahoei et al. 2010, 2012, 2013); Israel (Mescheloff \& Rosen 1988).

\section{General distribution}

Eastern (Iran) and Western Palaearctic.

Praon unitum Mescheloff \& Rosen, 1988

Figs 59, 160, 260, 359, 460, 597

\section{Host records}

Macrosiphoniella sanborni (Gillette) (Mescheloff \& Rosen 1988; Rakhshani et al. 2011); Uroleucon acroptilidis Kadyrbekov, Renxin \& Shao (Barahoei et al. 2010, 2012, 2013); Uroleucon jaceae (L.) (Barahoei et al. 2013); Uroleucon sonchi (L.) (Mescheloff \& Rosen 1988; Barahoei et al. 2013). 


\section{Distribution in the Middle East and North Africa}

Iran (Barahoei et al. 2010, 2012, 2013); Israel (Mescheloff \& Rosen 1988).

\section{General distribution}

Eastern (Iran) and Western Palaearctic (Israel).

Praon uroleucon Tomanović \& Kavallieratos, 2003

Figs 161, 261, 360, 461, 598

\section{Host records}

Lacking host data (Akar \& Erdoğan 2017).

\section{Distribution in the Middle East and North Africa}

Turkey (Akar \& Erdoğan 2017).

\section{General distribution}

Western Palaearctic (Southeastern Europe).

Praon volucre (Haliday, 1833)

Figs 60, 162, 262, 361, 462, 599

\section{Material examined}

SAUDI ARABIA • 9 Q $\bigcirc$; Abha; Jun. 2012; Acyrthosiphon pisum (Harris) on Trifolium alexandrinum • 3 ㅇ; A Abha-Mahala College campus; 28 Nov. 2012; Uroleocon sp. on Sonchus oleraceus 1 1 9 ; AbhaMahala College campus; Dec. 2012; Aphis solanella Thoebald on Solanum nigrum • 1 q; Abha-Mahala

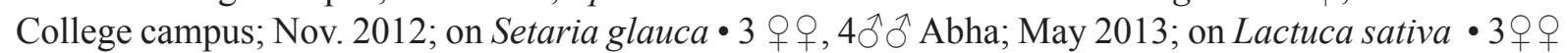

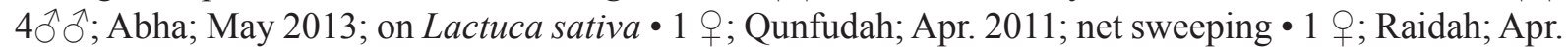
2011; Z. Ahmad leg (DPPZ).

\section{Host records}

Acyrthosiphon lactucae (Passerini) (Düzgüneş et al. 1982; Uysal et al. 2004; Rakhshani et al. 2007a); Acyrthosiphon pisum (Harris) (Zeren \& Düzgüneș 1983; Uysal et al. 2004; Rakhshani et al. 2006a, 2007a; Laamari et al. 2011, 2012; Chaouche \& Laamari 2015); Amphorophora catharinae (Nevsky) (Rakhshani et al. 2012b; Barahoei et al. 2010, 2012, 2013); Aphis affinis del Guercio (Rakhshani et al. 2012b); Aphis aurantii Boyer de Fonscolombe (Hussein \& Kawar 1984; Tremblay et al. 1985; Boukhris-Bouhachem 2011; Yoldaş et al. 2011); Aphis craccivora Koch (Zeren \& Düzgüneş 1983; Mescheloff \& Rosen 1988; Uysal et al. 2004; Rakhshani et al. 2005a, 2006a, 2007a; Barahoei et al. 2010; Yoldaş et al. 2011; Laamari et al. 2011, 2012; Barahoei et al. 2012, 2013; Rakhshani et al. 2012b); Aphis dlabolai Holman (Barahoei et al. 2013); Aphis epilobiaria Theobald (Mescheloff \& Rosen 1988); Aphis fabae Scopoli (Starý 1979; Abou-Fakhr 1982; Düzgüneş et al. 1982; Hussein \& Kawar 1984; Tremblay et al. 1985; Mescheloff \& Rosen 1988; Ben Halima et al. 1994 - as Aphis citricola van der Goot; Abou-Fakhr \& Kawar 1998; Starý et al. 2000; Tomanović et al. 2003; Kavallieratos et al. 2004; Uysal et al. 2004; Ben Halima \& Ben Hamouda 2005 - as Aphis citricola van der Goot; Güz \& Kilinçer 2005; Rakhshani et al. 2007a; Laamari et al. 2011, 2012; Nazari et al. 2012; Barahoei et al. 2013; Kavallieratos et al. 2013; Taheri \& Rakhshani 2013); Aphis gossypii Glover (Abou-Fakhr 1982; Hussein \& Kawar 1984; Tremblay et al. 1985; Yoldaş et al. 2011; Barahoei et al. 2013; Satar et al. 2014); Aphis nasturtii Kaltenbach (Mescheloff \& Rosen 1988 - as Aphis zizyphi Theobald); Aphis nerii Boyer de Fonscolombe (Tremblay et al. 1985; Abou-Fakhr \& Kawar 1998); Aphis pomi de Geer (Düzgüneş et al. 1982; Hussein \& Kawar 1984; Uysal et al. 2004; Rakhshani 2012); Aphis punicae Passerini (Hussein \& Kawar 1984); Aphis salviae Walker (Laamari 
et al. 2011, 2012); Aphis solanella Theobald (Düzgüneş et al. 1982; Uysal et al. 2004; Rakhshani et al. 2007a; Talebi et al. 2009; Barahoei et al. 2010, 2012, 2013); Aphis spiraecola Patch (Yoldaş et al. 2011; Boukhris-Bouhachem 2011); Aphis umbrella (Börner) (Tremblay et al. 1985; Abou-Fakhr \& Kawar 1998); Aphis urticata Gmelin (Rakhshani et al. 2012b); Brachycaudus amygdalinus (Schouteden) (Düzgüneş et al. 1982; Uysal et al. 2004; Rakhshani et al. 2007a; Talebi et al. 2009; Rakhshani 2012); Brachycaudus cardui (L.) (Düzgüneş et al. 1982; Uysal et al. 2004; Aslan \& Karaca 2005; Laamari et al. 2011, 2012; Rakhshani 2012); Brachycaudus helichrysi (Kaltenbach) (Tremblay et al. 1985; Rakhshani et al. 2007a; Talebi et al. 2009; Jafari et al. 2011; Jafari \& Modarres Awal 2012; Rakhshani 2012; Barahoei et al. 2013); Brachycaudus persicae (Passerini) (Rakhshani 2012); Brachycaudus tragopogonis (Kaltenbach) (Mescheloff \& Rosen 1988); Brachycaudus sp. (Mescheloff \& Rosen 1988); Brevicoryne brassicae (L.) (Kilinçer 1982; Uysal et al. 2004); Diuraphis noxia (Kurdjumov) (Pike et al. 1991; Starý et al. 2000; Uysal et al. 2004; Rakhshani et al. 2007a, 2008b; Barahoei et al. 2013); Dysaphis pyri (Boyer de Fonscolombe) (Rakhshani 2012); Hyalopterus amygdali (Blanchard) (Kavallieratos et al. 2004; Rakhshani et al. 2007a; Nazari et al. 2012; Taheri \& Rakhshani 2013; Rakhshani 2012); Hyalopterus pruni (Geoffroy) (Starý \& Kaddou 1971; Starý 1976; Düzgüneş et al. 1982; Hussein \& Kawar 1984; Tomanović et al. 2003; Kavallieratos et al. 2004; Ölmez \& Ulusoy 2003; Uysal et al. 2004; Ben Halima \& Ben Hamouda 2005; Aslan \& Karaca 2005; Güz \& Kilinçer 2005; Rakhshani et al. 2007a; Rakhshani 2012; Jafari et al. 2011; Jafari \& Modarres Awal 2012; Barahoei et al. 2013); Hyperomyzus lactucae (L.) (Abou-Fakhr 1982; Düzgüneş et al. 1982; Hussein \& Kawar 1984; Tremblay et al. 1985; Mescheloff \& Rosen 1988; Abou-Fakhr \& Kawar 1998; Tomanović et al. 2003; Uysal et al. 2004; Güz \& Kilinçer 2005; Rakhshani et al. 2007a; Talebi et al. 2009; Laamari et al. 2011, 2012; Chaouche \& Laamari 2015); Hyperomyzus picridis (Börner) (Laamari et al. 2011, 2012); Liosomaphis berberidis (Kaltenbach) (Laamari et al. 2011); Macrosiphum euphorbiae (Thomas) (Zeren \& Düzgüneş 1983; Mescheloff \& Rosen 1988; Yoldaş et al. 1990; Ben Halima \& Ben Hamouda 1993; Uysal et al. 2004); Macrosiphum rosae (L.) (Starý \& Kaddou 1971; Starý 1976; Abou-Fakhr 1982; Düzgüneş et al. 1982; Hussein \& Kawar 1984; Tremblay et al. 1985; Abou-Fakhr \& Kawar 1998; Starý \& Sekkat 1987; Uysal et al. 2004; Rakhshani et al. 2007a; Talebi et al. 2009; Barahoei et al. 2010, 2012, 2013; Laamari et al. 2011; Nazari et al. 2012; Kavallieratos et al. 2013); Macrosiphum sp. (Starý et al. 2000); Macrosiphoniella riedeli Szelegiewicz (Mescheloff \& Rosen 1988); Macrosiphoniella sp. (Rakhshani et al. 2011); Melanaphis donacis (Passerini) (Starý \& Kaddou 1971; Starý 1976); Metopolophium dirhodum (Walker) (Rakhshani et al. 2008b; Barahoei et al. 2013; Taheri \& Rakhshani 2013); Myzaphis rosarum (Kaltenbach) (Düzgüneş et al. 1982; Uysal et al. 2004); Myzus beybienkoi (Narzykulov) (Starý et al. 2000); Myzus persicae (Sulzer) (Rosen 1967, 1969; Avidov \& Harpaz 1969; Starý 1976, 1979; Abou-Fakhr 1982; Düzgüneş et al. 1982; Zeren \& Düzgüneş 1983; Hussein \& Kawar 1984; Tremblay et al. 1985; Starý \& Sekkat 1987; Mescheloff \& Rosen 1988; Ben Halima \& Ben Hamouda 1993; Ben Halima et al. 1994; Abou-Fakhr \& Kawar 1998; Starý et al. 2000; Uysal et al. 2004; Ben Halima \& Ben Hamouda 2005; Güz \& Kilinçer 2005; Rakhshani et al. 2007a; Rakhshani 2012; Jafari et al. 2011; Boukhris-Bouhachem 2011; Yoldaş et al. 2011; Laamari et al. 2012; Jafari \& Modarres Awal 2012; Nazari et al. 2012; Barahoei et al. 2013; Chaouche \& Laamari 2015); Phorodon humuli (Schrank) (Rakhshani et al. 2007a); Rhopalosiphum maidis (Fitch) (Hafez 1994; Güz \& Kilinçer 2005; Rakhshani et al. 2007a, 2008b; Nazari et al. 2012; Barahoei et al. 2010, 2012, 2013); Rhopalosiphum padi (L.) (Starý 1976; Hafez 1994; Özder \& Toros 1999; Uysal et al. 2004; Rakhshani et al. 2007a, 2008b; Barahoei et al. 2013; Gadallah et al. 2017); Schizaphis graminum (Rondani) (Mescheloff \& Rosen 1988; Hafez 1994; Rakhshani et al. 2007a, 2008b; Nazari et al. 2012; Barahoei et al. 2013); Sitobion avenae (Fabricius) (Düzgüneş et al. 1982; Özder \& Toros 1999; Ölmez \& Ulusoy 2003; Uysal et al. 2004; Rakhshani et al. 2007a, 2008b; Barahoei et al. 2013; Gadallah et al. 2017); Sitobion fragariae (Walker) (Starý 1976; Mescheloff \& Rosen 1988; Laamari et al. 2011, 2012); Uroleucon aeneum (Hille Ris Lambers) (Tomanović et al. 2003; Kavallieratos et al. 2004); Uroleucon cichorii (Koch) (Tremblay et al. 1985; Rakhshani et al. 2007a); Uroleucon compositae (Theobald) (Rakhshani et al. 2007a; Barahoei et al. 2013); Uroleucon inulicola (Hille Ris Lambers) (Abou-Fakhr \& Kawar 1998); Uroleucon jaceae (L.) (Rakhshani et al. 2007a); Uroleucon sonchi (L.) (Abou-Fakhr 1982; 
Tremblay et al. 1985; Abou-Fakhr \& Kawar 1998; Kavallieratos et al. 2004; Güz \& Kilinçer 2005; Rakhshani et al. 2007a, 2012b; Talebi et al. 2009; Nazari et al. 2012; Laamari et al. 2011, 2012; Barahoei et al. 2010, 2012, 2013); Uroleucon sp. (Chaouche \& Laamari 2015)

\section{Distribution in the Middle East and North Africa}

Algeria (Starý 1976; Laamari et al. 2011, 2012; Chaouche \& Laamari 2015); Egypt (Hafez 1994; ElGhiet et al. 2014; Gadallah et al. 2017); Iran (Starý 1979; Starý et al. 2000; Rakhshani et al. 2005a, 2006a, 2007a, 2012b; Jafari et al. 2011; Jafari \& Modarres Awal 2012; Rakhshani 2012; Nazari et al. 2012; Taheri \& Rakhshani 2013; Barahoei et al. 2010, 2012, 2013); Iraq (Starý \& Kaddou 1971; Starý 1976); Israel (Fleschner 1963; Rosen 1967, 1969; Avidov \& Harpaz 1969; Starý 1976; Mescheloff \& Rosen 1988); Lebanon (Abou-Fakhr 1982; Hussein \& Kawar 1984; Tremblay et al. 1985; AbouFakhr \& Kawar 1998); Morocco (Starý \& Sekkat 1987); Tunisia (Ben Halima \& Ben Hamouda 1993, 2005; Ben Halima et al. 1994; Boukhris-Bouhachem 2011); Turkey (Tomanović et al. 2003; Eastern part - Ölmez \& Ulusoy 2003; Central part - Düzgüneş et al. 1982; Zeren \& Düzgüneş 1983; Kilinçer 1982; Güz \& Kilinçer 2005; Satar et al. 2014; Western part - Yoldaş et al. 1990; Özder \& Toros 1999; Kavallieratos et al. 2004, 2013; Aslan \& Karaca 2005; Erdoğan et al. 2008; Yoldaş et al. 2011; Akar \& Erdoğan 2017).

\section{General distribution}

Eastern and Western Palaearctic, Neotropical, Oriental.

Praon yomenae Takada, 1968

Figs $61,163,263,362,463,600$

\section{Material examined}

SAUDI ARABIA • 1 đ̇; Abha; May 2013; Uroleucon sp. on Sonchus oleraceus $\bullet 5$ 우; Abha-Mahala College campus; Nov. 2012; Uroleucon sp. on Sonchus oleraceus; Z. Ahmad leg. (DPPZ).

\section{Host records}

Acyrthosiphon pisum (Harris) (?) (Chaouche \& Laamari 2015); Metopolophium dirhodum (Walker) (?) (Gülçlü \& Özbek 2002; Uysal et al. 2004); Uroleucon acroptilidis Kadyrbekov, Renxin \& Shao (Barahoei et al. 2010, 2012, 2013); Uroleucon aeneum Hille Ris Lambers (Laamari et al. 2012); Uroleucon carthami (Hille Ris Lambers) (Mescheloff \& Rosen 1988 - as Praon dorsale; Barahoei et al. 2013); Uroleucon cichorii (Koch) (Barahoei et al. 2010, 2012, 2013); Uroleucon chondrillae (Nevsky) (Rakhshani et al. 2007a; Nazari et al. 2012); Uroleucon compositae (Theobald) (Rakhshani et al. 2007a); Uroleucon jaceae (L.) (Güz \& Kilinçer 2005 - as Praon dorsale; Rakhshani et al. 2007a; Barahoei et al. 2010, 2012, 2013; Laamari et al. 2011, 2012; Chaouche \& Laamari 2015); Uroleucon picridis (Fabricius) (Mescheloff \& Rosen 1988 - as Praon dorsale); Uroleucon sonchi (L.) (Starý \& Sekkat 1987; Mescheloff \& Rosen 1988 - as Praon dorsale; Tomanović et al. 2003; Kavallieratos et al. 2004; Rakhshani et al. 2007a, 2012b; Talebi et al. 2009; Barahoei et al. 2010, 2012, 2013); Uroleucon tortuosissimae Rezwani \& Lampel (Barahoei et al. 2013).

\section{Distribution in the Middle East and North Africa}

Algeria (Laamari et al. 2011, 2012; Chaouche \& Laamari 2015); Iran (Rakhshani et al. 2007a, 2012b; Barahoei et al. 2010, 2012, 2013; Nazari et al. 2012); Israel (Mescheloff \& Rosen 1988); Morocco (Starý \& Sekkat 1987); Turkey (Eastern part - Gülçlü \& Özbek 2002; Tomanović et al. 2003; Central part - Güz \& Kilinçer 2005; Erdoğan et al. 2008; Western part - Kavallieratos et al. 2004; Uysal et al. 2004; Akar \& Erdoğan 2017); Yemen (Starý et al. 2013). 


\section{General distribution}

Eastern and Western Palaearctic, Oriental.

\section{Remarks}

All records for Praon dorsale (Haliday 1833) associated with Uroleucon aphids in the Middle East and North Africa refer to Praon yomenae. Record of P. yomenae on Acyrthosiphon pisum (Chaouche \& Laamari 2015), is probably referring to a misidentification of Praon barbatum Mackauer, which is very similar to P. yomane.

\section{Host records}

Tanytrichophorus petiolaris Mackauer, 1961

Brachycaudus persicae (Passerini) (Mackauer 1961; Starý 1979; Starý et al. 2000).

\section{Distribution in the Middle East and North Africa}

Tehran (Starý et al. 2000).

\section{General distribution}

The species has not been reported outside Iran.

\section{Remarks}

The species was described on the basis of male specimens only (Mackauer 1961), while no female specimens have been recorded since the original description. Therefore, it has been excluded from the key of the present study.

Toxares deltiger (Haliday, 1833)

Figs 62, 164, 264, 363, 464, 601

\section{Host records}

Lacking host data (Tomanović et al. 2008).

\section{Distribution in the Middle East and North Africa}

Turkey (Eastern part - Tomanović et al. 2008).

\section{General distribution}

Holarctic, Oriental.

Trioxys asiaticus Telenga, 1953

Figs 63, 165, 265, 364, 465, 602

\section{Host records}

Acyrthosiphon gossypii Mordvilko (Mackauer 1960; Starý 1979; Starý et al. 2000; Rakhshani et al. 2008c; Talebi et al. 2009; Barahoei et al. 2013); Acyrthosiphon pisum (Harris) (Barahoei et al. 2013).

\section{Distribution in the Middle East and North Africa}

Iran (Mackauer 1960; Starý 1979; Starý et al. 2000; Talebi et al. 2009; Barahoei et al. 2013).

\section{General distribution}

Eastern Palaearctic. 
Trioxys cirsii (Curtis, 1831)

Figs 64, 166, 266, 365, 466, 603

\section{Host records}

Drepanosiphum platanoidis (Schrank) (Babaee et al. 2000; Starý et al. 2000; Rakhshani et al. 2008c).

\section{Distribution in the Middle East and North Africa}

Iran (Babaee et al. 2000; Starý et al. 2000; Rakhshani et al. 2008c).

\section{General distribution}

Australasian, Eastern and Western Palaearctic, Nearctic.

Trioxys complanatus Quilis, 1931

Figs 65, 167, 267, 366, 467, 604

\section{Host records}

Therioaphis khayami Remaudière (Starý et al. 2000); Therioaphis riehmi (Börner) (van den Bosch 1957; Starý 1979; Starý et al. 2000); Therioaphis trifolii (Monell) (van den Bosch 1957; Farahbakhsh 1961; Avidov \& Harpaz 1969; Al-Azawi 1970; Starý 1976, 1979; Monajemi \& Esmaili 1981; Starý \& Erdelen 1982; Düzgüneş et al. 1982; Tremblay et al. 1985; Starý et al. 2000; Uysal et al. 2004; Rakhshani et al. 2006a, 2008c, 2012b; Nazari et al. 2012; Barahoei et al. 2013; Laamari et al. 2012; Starý et al. 2013); Therioaphis spp. (Starý 1976, 1979; Mescheloff \& Rosen 1993).

\section{Distribution in the Middle East and North Africa}

Algeria (Laamari et al. 2012); Iran (van den Bosch 1957; Farahbakhsh 1961; Starý 1979; Monajemi \& Esmaili 1981; Starý et al. 2000; Rakhshani et al. 2006a, 2012b; Nazari et al. 2012; Barahoei et al. 2013); Iraq (van den Bosch 1957; Al-Azawi 1970; Starý 1976); Israel (van den Bosch 1957; Starý 1976; Mescheloff \& Rosen 1993); Lebanon (Tremblay et al. 1985); Morocco (Starý 1976); Turkey (van den Bosch 1957; Avidov \& Harpaz 1969; Starý 1976; Central part - Düzgüneş et al. 1982; Uysal et al. 2004); UAE (Starý et al. 2013); Yemen (Starý \& Erdelen 1982; Starý et al. 2013).

\section{General distribution}

Cosmopolitan.

Trioxys curvicaudus Mackauer, 1967

Figs 66, 168, 367, 468, 605

\section{Host records}

Eucallipterus tiliae (L.) (Rakhshani et al. 2008c).

\section{Distribution in the Middle East and North Africa}

Iran (Rakhshani et al. 2008c).

\section{General distribution}

Eastern and Western Palaearctic, Nearctic. 
Trioxys moshei Mescheloff \& Rosen, 1993

Figs 68, 170, 369, 470, 607

\section{Host records}

Hoplocallis picta (Ferrari) (Mescheloff \& Rosen 1993 - as Myzocallis bodenheimeri Hille Ris Lambers).

\section{Distribution in the Middle East and North Africa}

Israel (Mescheloff \& Rosen 1993).

\section{General distribution}

This species has not been reported outside Israel.

Trioxys metacarpalis Rakhshani \& Starý, 2012

Figs 67, 169, 268, 368, 469, 606

\section{Host records}

Chaitaphis tenuicauda Nevsky (Rakhshani et al. 2012b).

\section{Distribution in the Middle East and North Africa}

Iran (Rakhshani et al. 2012b).

\section{General distribution}

This species has not been reported outside Iran.

Trioxys pallidus (Haliday, 1833)

Figs 69, 171, 269, 370, 471, 608

\section{Host records}

Chromaphis juglandicola (Kaltenbach) (van den Bosch et al. 1970; Starý \& Kaddou 1971; van den Bosch \& Messenger 1973; Starý 1976; Starý 1979; Starý \& Sekkat 1987; Mohammadbeigi 2000; Starý et al. 2000; Ölmez \& Ulusoy 2003; Aslan et al. 2004; Rakhshani et al. 2004, Uysal et al. 2004; Talebi et al. 2009; Laamari et al. 2011, 2012; Rakhshani et al. 2008c, 2012b; Barahoei et al. 2012, 2013; Nazari et al. 2012; Taheri \& Rakhshani 2013); Hoplocallis picta (Ferrari) (Rakhshani et al. 2008c); Monellia caryella (Fitch) (Mescheloff \& Rosen 1993 - as Monellia costalis (Fitch)); Pterocallis alni (De Geer) (Babaee et al. 2000; Starý et al. 2000); Tuberculatus (Tuberculoides) albosiphonatus Hille Ris Lambers (Starý 1976); Tuberculatus (Tuberculoides) moerickei Hille Ris Lambers (Starý 1976); Tuberculatus sp. (Starý 1969b; Starý \& Kaddou 1971).

\section{Distribution in the Middle East and North Africa}

Algeria (Laamari et al. 2011, 2012); Iran (van den Bosch et al. 1970; van den Bosch \& Messenger 1973; Starý 1979; Mohammadbeigi 2000; Starý et al. 2000; Rakhshani et al. 2004; Barahoei et al. 2012, 2013; Nazari et al. 2012; Taheri \& Rakhshani 2013); Iraq (Starý 1969b, 1976; Starý \& Kaddou 1971); Israel (Mescheloff \& Rosen 1993); Morocco (Starý \& Sekkat 1987); Turkey (Central part - Starý 1976; Aslan et al. 2004; Uysal et al. 2004; Eastern part - Ölmez \& Ulusoy 2003).

\section{General distribution}

Eastern and Western Palaearctic, Nearctic (imported), Neotropical (imported), Oriental. 
Trioxys pannonicus Starý, 1960

Figs 70, 172, 270, 371, 472, 609

\section{Host records}

Macrosiphoniella tuberculata (Nevsky) (Starý 1979; Starý et al. 2000; Rakhshani et al. 2011); Macrosiphoniella sp. (Rakhshani et al. 2008c; Talebi et al. 2009); Titanosiphon neoartemisiae (Takahashi) (Barahoei et al. 2014)

\section{Distribution in the Middle East and North Africa}

Iran (Talebi et al. 2009; Starý 1979; Starý et al. 2000; Barahoei et al. 2014).

\section{General distribution}

Eastern and Western Palaearctic, Oriental.

\section{Trioxys pappi Takada, 1979 \\ Figs 71, 173, 473, 610}

\section{Host records}

Lacking host data (Davidian 2005).

\section{Distribution in the Middle East and North Africa}

Iran (Davidian 2005) - as Trioxys persicus Davidian.

\section{General distribution}

Eastern Palaearctic (Iran, Mongolia).

\section{Trioxys quercicola Starý, 1969}

Figs 174, 372, 474, 611

\section{Host records}

Thelaxes suberi (Del Guercio) (Starý 1969b, 1976; Starý \& Kaddou 1971).

\section{Distribution in the Middle East and North Africa}

Iraq (Starý 1969b, 1976; Starý \& Kaddou 1971).

\section{General distribution}

This species has not been reported outside Iraq.

Trioxys tanaceticola Starý, 1971

Figs $72,175,271,373,475,612$

\section{Host records}

Coloradoa heinzei Börner (Starý 1979; Starý et al. 2000); Coloradoa absinthii (Lichtenstein) (Talebi et al. 2009); Coloradoa sp. (Barahoei et al. 2014); Titanosiphon neoartemisiae (Takahashi) (?) (Rakhshani et al. 2008c; Talebi et al. 2009).

\section{Distribution in the Middle East and North Africa}

Iran (Starý 1979; Starý et al. 2000; Rakhshani et al. 2008c; Talebi et al. 2009). 


\section{General distribution}

Eastern and Western Palaearctic.

\section{Host records}

\section{Trioxys spp.}

Aphis craccivora Koch (?) (Abdel-Samad \& Ahmed 2009); Hyadaphis coriandri (Das) (Chaouche \& Laamari 2015).

\section{Distribution in the Middle East and North Africa}

Algeria (Chaouche \& Laamari 2015); Egypt (El-Heneidy 1991, 1994; El-Heneidy \& Abdel-Samad 2001; El-Heneidy et al. 2001, 2002; Abdel-Rahman 2005; Abdel-Samad \& Ahmed 2009).

\section{Remarks}

These species may belong to genus Binodoxys, on the basis of host range patterns.

\section{Host aphid-parasitoid associations}

Absinthaphis sp.: Ephedrus persicae Froggatt.

Acyrthosiphon gossypii Mordvilko: Aphidius colemani Viereck [- platensis Brèthes, in part]; Aphidius ervi Haliday; Aphidius matricariae Haliday (?); Aphidius urticae Haliday; Lysiphlebus fabarum (Marshall) (?); Lysiphlebus testaceipes (Cresson); Trioxys asiaticus Telenga.

Acyrthosiphon ilka Mordvilko: Lysiphlebus fabarum (Marshall) (?); Aphidius ervi Haliday.

Acyrthosiphon kondoi Shinji: Aphidius ervi Haliday; Aphidius smithi Sharma \& Subba Rao.

Acyrthosiphon lactucae (Passerini): Aphidius ervi Haliday; Lysiphlebus fabarum (Marshall) (?); Praon volucre (Haliday).

Acyrthosiphon malvae (Mosley): Ephedrus persicae Froggatt.

Acyrthosiphon pisum (Harris): Aphidius avenae Haliday; Aphidius banksae Kittel; Aphidius colemani Viereck [- platensis Brèthes, in part] (?); Aphidius eadyi Starý, González \& Hall; Aphidius ervi Haliday; Aphidius smithi Sharma \& Subba Rao; Aphidius urticae Haliday; Binodoxys acalephae (Marshall) (?); Ephedrus persicae Froggatt; Ephedrus plagiator (Nees); Lysiphlebus fabarum (Marshall) (?); Praon barbatum Mackauer; Praon exsoletum (Nees) (?); Praon volucre (Haliday); Praon yomenae Takada (?); Trioxys asiaticus Telenga.

Acyrthosiphon sp.: Aphidius urticae Haliday; Lysiphlebus confusus Tremblay \& Eady (?).

Amegosiphon platicaudum (Narzikulov): Aphidius matricariae Haliday; Aphidius platensis Brèthes; Diaeretiella rapae (M'Intosh).

Amphorophora catharinae (Nevsky): Aphidius popovi Starý; Praon volucre (Haliday).

Anuraphis sp.: Binodoxys acalephae (Marshall).

Aphis acetosae L.: Aphidius platensis Brèthes; Binodoxys angelicae (Haliday); Lysiphlebus fabarum (Marshall).

Aphis affinis Del Guercio: Aphidius matricariae Haliday; Aphidius platensis Brèthes; Binodoxys acalephae (Marshall); Binodoxys angelicae (Haliday); Ephedrus persicae Froggatt; Lysiphlebus confusus Tremblay \& Eady; Lysiphlebus fabarum (Marshall); Praon volucre (Haliday).

Aphis althaeae (Nevsky): Lysiphlebus fabarum (Marshall). 
Aphis astragali Ossiannilsson: Lysiphlebus fabarum (Marshall); Lysiphlebus testaceipes (Cresson).

Aphis aurantii Boyer de Fonscolombe: Aphidius colemani Viereck [- platensis Brèthes, in part]; Aphidius matricariae Haliday; Binodoxys angelicae (Haliday); Diaeretiella rapae (M'Intosh); Ephedrus persicae Froggatt; Lysiphlebus confusus Tremblay \& Eady; Lysiphlebus fabarum (Marshall); Lysiphlebus testaceipes (Cresson); Praon volucre (Haliday).

Aphis brunnea Ferrari: Ephedrus persicae Froggatt; Lysiphlebus fabarum (Marshall); Lysiphlebus testaceipes (Cresson).

Aphis chloris Koch: Aphidius colemani Viereck [- platensis Brèthes, in part]; Ephedrus persicae Froggatt; Lysiphlebus fabarum (Marshall).

Aphis craccivora Koch: Adialytus veronicaecola (Starý); Aphidius colemani Viereck [- platensis Brèthes, in part]; Aphidius ervi Haliday; Aphidius matricariae Haliday; Aphidius platensis Brèthes; Binodoxys acalephae (Marshall); Binodoxys angelicae (Haliday); Diaeretiella rapae (M'Intosh); Ephedrus persicae Froggatt; Lysiphlebus confusus Tremblay \& Eady; Lysiphlebus desertorum Starý; Lysiphlebus fabarum (Marshall); Lysiphlebus testaceipes (Cresson); Praon abjectum (Haliday); Praon necans Mackauer; Praon volucre (Haliday); Trioxys sp. (?).

Aphis crepidis (Börner): Aphidius matricariae Haliday.

Aphis davletshinae Hillie Ris Lambers: Lysiphlebus fabarum (Marshall).

Aphis dlabolai Holman: Aphidius matricariae Haliday; Praon volucre (Haliday).

Aphis epilobiaria Theobald: Aphidius colemani Viereck [- platensis Brèthes, in part]; Binodoxys angelicae (Haliday); Ephedrus persicae Froggatt; Lysiphlebus fabarum (Marshall); Praon volucre (Haliday).

Aphis epilobii Kaltenbach: Lysiphlebus fabarum (Marshall).

Aphis euphorbiae Kaltenbach: Aphidius matricariae Haliday; Binodoxys acalephae (Marshall); Lysiphlebus confusus Tremblay \& Eady; Lysiphlebus fabarum (Marshall); Lysiphlebus testaceipes (Cresson).

Aphis euphorbicola Rezwani \& Lampel: Lysiphlebus fabarum (Marshall).

Aphis fabae cirsiiacanthoidis Scopoli: Binodoxys acalephae (Marshall); Binodoxys angelicae (Haliday); Ephedrus persicae Froggatt; Lysiphlebus fabarum (Marshall).

Aphis fabae Scopoli: Aphidius colemani Viereck [- platensis Brèthes, in part]; Aphidius ervi Haliday; Aphidius funebris Mackauer (?); Aphidius matricariae Haliday; Aphidius platensis Brèthes; Binodoxys acalephae (Marshall); Binodoxys angelicae (Haliday); Diaeretiella rapae (M'Intosh); Ephedrus persicae Froggatt; Ephedrus plagiator (Nees); Lipolexis gracilis Förster; Lysiphlebus cardui (Marshall); Lysiphlebus confusus Tremblay \& Eady; Lysiphlebus fabarum (Marshall); Lysiphlebus testaceipes (Cresson); Praon volucre (Haliday).

Aphis farinosa Gmelin: Lysiphlebus confusus Tremblay \& Eady.

Aphis galiiscabri Schrank: Binodoxys acalephae (Marshall); Lysiphlebus fabarum (Marshall).

Aphis gerardianae Mordvilko: Lysiphlebus fabarum (Marshall).

Aphis gossypii Glover: Lysiphlebus cardui (Marshall) (?); Adialytus veronicaecola (Starý); Aphidius colemani Viereck [- platensis Brèthes, in part]; Aphidius matricariae Haliday; Aphidius platensis Brèthes; Binodoxys acalephae (Marshall); Binodoxys angelicae (Haliday); Diaeretiella rapae (M'Intosh); Ephedrus persicae Froggatt; Lipolexis gracilis Förster; Lysiphlebus confusus Tremblay \& 
Eady; Lysiphlebus fabarum (Marshall); Lysiphlebus testaceipes (Cresson); Praon necans Mackauer; Praon volucre (Haliday).

Aphis grossulariae Kaltenbach: Binodoxys acalephae (Marshall).

Aphis hederae Kaltenbach: Aphidius colemani Viereck [- platensis Brèthes, in part]; Aphidius matricariae Haliday; Binodoxys angelicae (Haliday); Ephedrus persicae Froggatt; Lysiphlebus confusus Tremblay \& Eady; Lysiphlebus fabarum (Marshall).

Aphis idaei van der Goot: Binodoxys acalephae (Marshall); Lysiphlebus confusus Tremblay \& Eady; Lysiphlebus fabarum (Marshall).

Aphis illinoisensis Shimer: Aphidius colemani Viereck [- platensis Brèthes, in part]; Aphidius matricariae Haliday; Diaeretiella rapae (M’Intosh); Lysiphlebus testaceipes (Cresson).

Aphis intybi Koch: Aphidius colemani Viereck [- platensis Brèthes, in part]; Aphidius matricariae Haliday; Aphidius platensis Brèthes; Binodoxys angelicae (Haliday); Ephedrus persicae Froggatt; Lysiphlebus confusus Tremblay \& Eady; Lysiphlebus fabarum (Marshall).

Aphis nasturtii Kaltenbach: Aphidius colemani Viereck [- platensis Brèthes, in part]; Aphidius matricariae Haliday; Aphidius transcaspicus Telenga (?); Binodoxys angelicae (Haliday); Ephedrus persicae Froggatt; Lysiphlebus confusus Tremblay \& Eady; Lysiphlebus confusus Tremblay \& Eady; Lysiphlebus fabarum (Marshall); Praon volucre (Haliday).

Aphis nerii Boyer de Fonscolombe: Aphidius colemani Viereck [- platensis Brèthes, in part]; Aphidius matricariae Haliday; Aphidius platensis Brèthes; Binodoxys acalephae (Marshall); Binodoxys angelicae (Haliday); Diaeretiella rapae (M'Intosh); Ephedrus persicae Froggatt; Lysiphlebus confusus Tremblay \& Eady; Lysiphlebus fabarum (Marshall); Lysiphlebus testaceipes (Cresson); Praon necans Mackauer; Praon volucre (Haliday).

Aphis origani Passerini: Lysiphlebus fabarum (Marshall).

Aphis parietariae Theobald: Aphidius matricariae Haliday.

Aphis plantaginis Goeze: Aphidius matricariae Haliday; Aphidius platensis Brèthes; Ephedrus persicae Froggatt; Lysiphlebus fabarum (Marshall).

Aphis polygonata (Nevsky): Binodoxys angelicae (Haliday).

Aphis pomi De Geer: Aphidius colemani Viereck [- platensis Brèthes, in part]; Aphidius matricariae Haliday; Binodoxys acalephae (Marshall); Binodoxys angelicae (Haliday); Ephedrus persicae Froggatt; Ephedrus plagiator (Nees); Lipolexis gracilis Förster; Lysiphlebus fabarum (Marshall); Lysiphlebus testaceipes (Cresson); Praon volucre (Haliday).

Aphis potentillae Nevsky: Lysiphlebus confusus Tremblay \& Eady; Lysiphlebus testaceipes (Cresson).

Aphis punicae (Passerini): Aphidius colemani Viereck [- platensis Brèthes, in part]; Aphidius matricariae Haliday; Aphidius matricariae Haliday; Aphidius platensis Brèthes; Binodoxys acalephae (Marshall); Binodoxys angelicae (Haliday); Diaeretiella rapae (M'Intosh); Ephedrus persicae Froggatt; Lysiphlebus confusus Tremblay \& Eady; Lysiphlebus fabarum (Marshall); Lysiphlebus testaceipes (Cresson); Praon abjectum (Haliday); Praon necans Mackauer; Praon volucre (Haliday).

Aphis ruborum (Börner): Binodoxys acalephae (Marshall); Binodoxys angelicae (Haliday); Ephedrus persicae Froggatt; Lysiphlebus confusus Tremblay \& Eady; Lysiphlebus fabarum (Marshall).

Aphis rumicis L.: Aphidius platensis Brèthes; Binodoxys angelicae (Haliday); Lysiphlebus fabarum (Marshall). 
Aphis salviae Walker: Lipolexis gracilis Förster; Lysiphlebus fabarum (Marshall); Praon volucre (Haliday).

Aphis solanella Theobald: Aphidius colemani Viereck [- platensis Brèthes, in part]; Aphidius matricariae Haliday; Aphidius platensis Brèthes; Aphidius platensis Brèthes; Binodoxys angelicae (Haliday); Diaeretiella rapae (M'Intosh); Lysiphlebus confusus Tremblay \& Eady; Lysiphlebus fabarum (Marshall); Lysiphlebus testaceipes (Cresson); Praon abjectum (Haliday); Praon volucre (Haliday).

Aphis spiraecola Patch: Aphidius matricariae Haliday; Binodoxys acalephae (Marshall); Binodoxys angelicae (Haliday); Diaeretiella rapae (M'Intosh); Lysiphlebus fabarum (Marshall); Lysiphlebus testaceipes; Praon volucre (Haliday).

Aphis taraxacicola (Börner): Lysiphlebus fabarum (Marshall).

Aphis tirucallis Hille Ris Lambers: Lysiphlebus fabarum (Marshall).

Aphis umbrella (Börner): Aphidius colemani Viereck [- platensis Brèthes, in part]; Aphidius matricariae Haliday; Aphidius platensis Brèthes; Binodoxys acalephae (Marshall); Binodoxys angelicae (Haliday); Diaeretiella rapae (M'Intosh); Ephedrus persicae Froggatt; Lipolexis gracilis Förster; Lysiphlebus confusus Tremblay \& Eady; Lysiphlebus fabarum (Marshall); Praon volucre (Haliday).

Aphis urticata Gmelin: Binodoxys acalephae (Marshall); Lysiphlebus confusus Tremblay \& Eady; Lysiphlebus fabarum (Marshall); Praon volucre (Haliday).

Aphis vallei Hille Ris Lambers \& Stroyan: Binodoxys acalephae (Marshall); Lysiphlebus confusus Tremblay \& Eady.

Aphis verbasci Schrank: Aphidius colemani Viereck [- platensis Brèthes, in part]; Ephedrus persicae Froggatt; Lysiphlebus confusus Tremblay \& Eady; Lysiphlebus fabarum (Marshall); Lysiphlebus volkli Tomanović \& Kavallieratos.

Aphis viticis Ferrari: Binodoxys angelicae (Haliday); Praon abjectum (Haliday).

Aphis spp.: Aphidius avenae Haliday; Aphidius colemani Viereck [- platensis Brèthes, in part]; Binodoxys acalephae (Marshall); Binodoxys angelicae (Haliday); Lipolexis gracilis Förster; Lysiphlebus desertorum Starý; Lysiphlebus fabarum (Marshall); Praon abjectum (Haliday).

Aspidaphis adjuvans (Walker): Diaeretiella rapae (M'Intosh).

Aulacorthum solani (Kaltenbach): Aphidius matricariae Haliday.

Aulacorthum sp.: Lysiphlebus confusus Tremblay \& Eady.

Brachycaudus amygdalinus (Schouteden): Aphidius colemani Viereck [- platensis Brèthes, in part]; Aphidius matricariae Haliday; Aphidius platensis Brèthes; Aphidius transcaspicus Telenga (?); Diaeretiella rapae (M'Intosh); Ephedrus persicae Froggatt; Lipolexis gracilis Förster; Lysiphlebus confusus Tremblay \& Eady; Lysiphlebus fabarum (Marshall); Praon volucre (Haliday).

Brachycaudus cardui (L.): Aphidius colemani Viereck [- platensis Brèthes, in part]; Aphidius funebris Mackauer (?); Aphidius matricariae Haliday; Aphidius platensis Brèthes; Diaeretiella rapae (M'Intosh); Ephedrus persicae Froggatt; Ephedrus plagiator (Nees); Lysiphlebus confusus Tremblay \& Eady; Lysiphlebus fabarum (Marshall); Lysiphlebus testaceipes (Cresson); Praon abjectum (Haliday); Praon volucre (Haliday).

Brachycaudus divaricatae Shaposhnikov: Aphidius matricariae Haliday.

Brachycaudus helichrysi (Kaltenbach): Aphidius colemani Viereck [- platensis Brèthes, in part]; Aphidius matricariae Haliday; Aphidius platensis Brèthes; Binodoxys angelicae (Haliday); Diaeretiella rapae 
(M'Intosh); Ephedrus persicae Froggatt; Ephedrus plagiator (Nees); Lysiphlebus confusus Tremblay \& Eady; Lysiphlebus fabarum (Marshall); Lysiphlebus testaceipes (Cresson); Praon volucre (Haliday).

Brachycaudus persicae (Passerini): Aphidius matricariae Haliday; Lysiphlebus fabarum (Marshall); Praon volucre (Haliday); Tanytrichophorus petiolaris Mackauer.

Brachycaudus tragopogonis (Kaltenbach): Aphidius matricariae Haliday; Aphidius platensis Brèthes; Lysiphlebus volkli Tomanović \& Kavallieratos; Praon volucre (Haliday).

Brachycaudus tragopogonis setosus (Hille Ris Lambers): Lysiphlebus fabarum (Marshall).

Brachycaudus sp.: Praon volucre (Haliday).

Brachyunguis harmalae Das: Lysiphlebus fabarum (Marshall).

Brachyunguis skafi Remaudière \& Talhouk: Lysiphlebus confusus Tremblay \& Eady.

Brachyunguis tamaricis (Lichtenstein): Ephedrus persicae Froggatt.

Brachyunguis zygophylli (Nevsky): Lysiphlebus fabarum (Marshall).

Brevicoryne brassicae (L.): Aphidius matricariae Haliday (?); Diaeretiella rapae (M'Intosh); Lysiphlebus testaceipes (Cresson); Praon volucre (Haliday).

Capitophorus elaeagni (Del Guercio): Aphidius colemani Viereck [- platensis Brèthes, in part]; Aphidius ervi Haliday; Aphidius matricariae Haliday; Aphidius platensis Brèthes; Lysiphlebus fabarum (Marshall).

Capitophorus inulae (Passerini): Aphidius matricariae Haliday; Diaeretiella rapae (M'Intosh).

Cavariella aegopodii (Scopoli): Aphidius salicis Haliday; Binodoxys brevicornis (Haliday); Ephedrus persicae Froggatt; Lysiphlebus fabarum (Marshall).

Cavariella aquatica (Gillette \& Bragg): Aphidius salicis Haliday; Ephedrus helleni Mackauer.

Cavariella aspidaphoides Hille Ris Lambers: Aphidius salicis Haliday; Binodoxys heraclei (Haliday).

Cavariella theobaldi (Gillette \& Bragg): Aphidius salicis Haliday; Binodoxys heraclei (Haliday); Ephedrus persicae Froggatt.

Chaetosiphon tetrarhodum (Walker): Aphidius eglanteriae Haliday.

Chaitaphis tenuicauda Nevsky: Trioxys metacarpalis Rakhshani \& Starý.

Chaitophorus euphraticus Hodjat: Adialytus salicaphis (Fitch).

Chaitophorus leucomelas Koch: Adialytus salicaphis (Fitch).

Chaitophorus populeti (Panzer): Adialytus salicaphis (Fitch); Ephedrus chaitophori Gärdenfors.

Chaitophorus populialbae (Boyer de Fonscolombe): Adialytus salicaphis (Fitch).

Chaitophorus remaudierei Pintera: Adialytus salicaphis (Fitch).

Chaitophorus salijaponicus Essig \& Kuwana: Adialytus salicaphis (Fitch).

Chaitophorus truncatus (Hausmann): Adialytus salicaphis (Fitch).

Chaitophorus vitellinae (Schrank): Adialytus salicaphis (Fitch).

Chaitophorus spp.: Adialytus salicaphis (Fitch).

Chromaphis juglandicola (Kaltenbach): Trioxys pallidus (Haliday).

Cinara (Cedrobium) laportei Remaudière: Pauesia cedrobii Starý \& Leclant. 
Cinara (Cupressobium) tujafilina (del Guercio): Pauesia hazratbalensis Bhagat.

Cinara (Schizolachnus) pineti (Fabricius): Pauesia unilachni (Gahan).

Cinara cedri Mimeur: Pauesia anatolica Michelena, Assael \& Mendel; Pauesia sp.

Cinara palaestinensis Hille Ris Lambers: Pauesia pini (Haliday); Pauesia silana Tremblay.

Cinara pilicornis (Hartig): Pauesia abietis (Marshall).

Cinara pini (L.): Pauesia picta (Haliday).

Cinara pinimaritimae (Dufour): Pauesia abietis (Marshall).

Coloradoa absinthii (Lichtenstein): Trioxys tanaceticola Starý.

Coloradoa achilleae Hille Ris Lambers: Aphidius arvensis (Starý).

Coloradoa heinzei Börner: Trioxys tanaceticola Starý.

Coloradoa sp.: Lysiphlebus fabarum (Marshall); Trioxys tanaceticola Starý.

Corylobium avellanae (Schrank): Ephedrus plagiator (Nees).

Cryptomyzus ribis (L.): Aphidius ribis Haliday.

Diuraphis noxia (Kurdjumov): Aphidius colemani Viereck [- platensis Brèthes, in part]; Aphidius matricariae Haliday; Aphidius platensis Brèthes; Aphidius rhopalosiphi De Stefani-Perez; Aphidius uzbekistanicus Luzhetzki; Diaeretiella rapae (M'Intosh); Ephedrus plagiator (Nees); Lysiphlebus testaceipes (Cresson); Praon volucre (Haliday).

Drepanosiphum platanoidis (Schrank): Trioxys cirsii (Curtis).

Dysaphis crataegi (Kaltenbach): Aphidius matricariae Haliday; Ephedrus persicae Froggatt; Ephedrus plagiator (Nees); Lysiphlebus confusus Tremblay \& Eady.

Dysaphis devecta (Walker): Aphidius matricariae Haliday; Ephedrus persicae Froggatt; Ephedrus plagiator (Nees).

Dysaphis foeniculus (Theobald): Aphidius ervi Haliday; Aphidius matricariae Haliday; Diaeretiella rapae (M'Intosh); Lysiphlebus confusus Tremblay \& Eady; Lysiphlebus testaceipes (Cresson); Ephedrus persicae Froggatt.

Dysaphis lappae (Koch): Aphidius matricariae Haliday; Lysiphlebus fabarum (Marshall).

Dysaphis plantaginea (Passerini): Aphidius matricariae Haliday; Ephedrus cerasicola Starý; Ephedrus persicae Froggatt; Ephedrus plagiator (Nees); Lysiphlebus fabarum (Marshall); Lysiphlebus testaceipes (Cresson).

Dysaphis pulverina ssp. iranica Stroyan: Aphidius platensis Brèthes.

Dysaphis pyri (Boyer de Fonscolombe): Aphidius matricariae Haliday; Diaeretiella rapae (M'Intosh); Ephedrus persicae Froggatt; Ephedrus plagiator (Nees); Lysiphlebus testaceipes (Cresson); Praon volucre (Haliday).

Dysaphis radicola (Mordvilko): Aphidius platensis Brèthes; Lysiphlebus fabarum (Marshall).

Dysaphis reaumuri (Mordvilko): Ephedrus persicae Froggatt.

Dysaphis rumecicola emicis (Mimeur): Lysiphlebus fabarum (Marshall).

Dysaphis tulipae (Boyer de Fonscolombe): Diaeretiella rapae (M'Intosh); Lysiphlebus testaceipes (Cresson).

Dysaphis spp.: Aphidius ervi Haliday; Ephedrus plagiator (Nees).

Ephedraphis ephedrae (Nevsky): Lysiphlebus confusus Tremblay \& Eady.

Eriosoma lanuginosum (Hartig): Areopraon lepelleyi (Waterson). 
Eucallipterus tiliae (L.): Trioxys curvicaudus Mackauer.

Eucarazzia elegans (Ferrari): Aphidius matricariae Haliday.

Eulachnus tuberculostemmatus (Theobald): Diaeretus leucopterus (Haliday); Ephedrus persicae Froggatt (?).

Forda sp.: Monoctonia pistaciaecola Starý.

Hayhurstia atriplicis (L.): Aphidius colemani Viereck [- platensis Brèthes, in part]; Aphidius platensis Brèthes; Diaeretiella rapae (M'Intosh); Ephedrus helleni Mackauer; Ephedrus nacheri Quilis; Ephedrus persicae Froggatt; Lysiphlebus fabarum (Marshall).

Hoplocallis picta (Ferrari): Trioxys moshei Mescheloff \& Rosen; Trioxys pallidus (Haliday).

Hyadaphis coriandri (Das): Binodoxys acalephae (Marshall); Binodoxys brevicornis (Haliday); Diaeretiella rapae (M'Intosh); Ephedrus persicae Froggatt; Lysiphlebus fabarum (Marshall); Trioxys sp.

Hyadaphis foeniculi (Passerini): Aphidius colemani Viereck [- platensis Brèthes, in part]; Aphidius salicis Haliday; Binodoxys brevicornis (Haliday); Diaeretiella rapae (M'Intosh); Ephedrus persicae Froggatt; Lysiphlebus confusus Tremblay \& Eady; Lysiphlebus fabarum (Marshall).

Hyadaphis tataricae (Aizenberg): Diaeretiella rapae (M'Intosh).

Hyadaphis sp.: Binodoxys brevicornis (Haliday).

Hyalopterus amygdali (Blanchard): Aphidius matricariae Haliday (?); Aphidius platensis Brèthes; Aphidius transcaspicus Telenga; Ephedrus persicae Froggatt; Lysiphlebus fabarum (Marshall) (?); Praon volucre (Haliday).

Hyalopterus pruni (Geoffroy): Aphidius matricariae Haliday (?); Aphidius transcaspicus Telenga; Ephedrus persicae Froggatt; Ephedrus plagiator (Nees); Lysiphlebus fabarum (Marshall) (?); Lysiphlebus testaceipes (Cresson); Praon volucre (Haliday).

Hyperomyzus lactucae (L.): Aphidius colemani Viereck [- platensis Brèthes, in part] (?); Aphidius funebris Mackauer (?); Aphidius matricariae Haliday (?); Aphidius avenae Haliday; Aphidius ervi Haliday; Aphidius sonchi Marshall; Diaeretiella rapae (M'Intosh); Lysiphlebus confusus Tremblay \& Eady (?); Praon orpheusi Kavallieratos, Athanassiou \& Tomanović; Praon volucre (Haliday).

Hyperomyzus picridis (Börner): Praon volucre (Haliday).

Liosomaphis berberidis (Kaltenbach): Praon volucre (Haliday).

Lipaphis erysimi (Kaltenbach): Diaeretiella rapae (M'Intosh); Lysiphlebus fabarum (Marshall); Aphidius matricariae Haliday.

Lipaphis fritzmuelleri Börner: Lysiphlebus fabarum (Marshall).

Lipaphis lepidii (Nevsky): Aphidius matricariae Haliday; Diaeretiella rapae (M'Intosh); Lysiphlebus fabarum (Marshall).

Lipaphis pseudobrassicae (Davis): Aphidius ervi Haliday; Aphidius matricariae Haliday; Diaeretiella rapae (M'Intosh).

Lipaphis sp.: Diaeretiella rapae (M'Intosh).

Macchiatiella rhamni (Boyer de Fonscolombe): Ephedrus persicae Froggatt.

Macrosiphoniella abrotani (Walker): Aphidius absinthii Marshall; Ephedrus niger Gautier, Bonnamour \& Gaumont. 
Macrosiphoniella absinthii (L.): Praon absinthii Bignell.

Macrosiphoniella artemisiae (Boyer de Fonscolombe): Aphidius absinthii Marshall.

Macrosiphoniella helichrysi Remaudière: Aphidius absinthii Marshall.

Macrosiphoniella nr. macrura Hille Ris Lambers: Aphidius absinthii Marshall.

Macrosiphoniella oblonga (Mordvilko): Aphidius absinthii Marshall; Praon absinthii Bignell.

Macrosiphoniella papillata Holman: Lysiphlebus fabarum (Marshall) (?).

Macrosiphoniella pulvera (Walker): Aphidius absinthii Marshall.

Macrosiphoniella riedeli Szelegiewicz: Aphidius absinthii Marshall; Praon volucre (Haliday).

Macrosiphoniella sanborni (Gillette): Diaeretiella rapae (M'Intosh); Ephedrus niger Gautier, Bonnamour \& Gaumont; Lysiphlebus fabarum (Marshall) (?); Praon absinthii Bignell; Praon necans Mackauer; Praon unitum Mescheloff \& Rosen.

Macrosiphoniella tanacetaria (Kaltenbach): Aphidius stigmaticus Rakhshani \& Tomanović.

Macrosiphoniella tapuskae (Hottes \& Frison): Lysiphlebus confusus Tremblay \& Eady (?).

Macrosiphoniella tuberculata (Nevsky): Aphidius absinthii Marshall; Trioxys pannonicus Starý.

Macrosiphoniella spp.: Aphidius absinthii Marshall; Aphidius asteris Haliday; Aphidius persicus Rakhshani \& Starý; Ephedrus niger Gautier, Bonnamour \& Gaumont; Praon volucre (Haliday); Trioxys pannonicus Starý.

Macrosiphum euphorbiae (Thomas): Aphidius colemani Viereck [- platensis Brèthes, in part]; Aphidius ervi Haliday; Aphidius matricariae Haliday; Aphidius platensis Brèthes; Aphidius urticae Haliday; Lysiphlebus testaceipes (Cresson); Praon volucre (Haliday).

Macrosiphum rosae (L.): Aphidius colemani Viereck [- platensis Brèthes, in part] (?); Aphidius platensis Brèthes (?); Aphidius matricariae Haliday; Aphidius rosae Haliday; Ephedrus niger Gautier, Bonnamour \& Gaumont (?); Lysiphlebus fabarum (Marshall) (?); Praon rosaecola Starý; Praon volucre (Haliday).

Macrosiphum spp.: Ephedrus plagiator (Nees); Praon volucre (Haliday).

Neomariaella lambersi (Szelegiewicz): Diaeretiella rapae (M'Intosh).

Melanaphis donacis (Passerini): Aphidius transcaspicus Telenga; Ephedrus persicae Froggatt; Praon volucre (Haliday).

Melanaphis sacchari (Zehntner): Lysiphlebus fabarum (Marshall).

Melanaphis sp.: Lysiphlebus confusus Tremblay \& Eady; Lysiphlebus fabarum (Marshall).

Metopolophium dirhodum (Walker): Aphidius ervi Haliday; Aphidius matricariae Haliday; Aphidius platensis Brèthes; Aphidius popovi Starý; Aphidius rhopalosiphi De Stefani-Perez; Aphidius uzbekistanicus Luzhetzki; Diaeretiella rapae (M'Intosh); Ephedrus persicae Froggatt; Lysiphlebus fabarum (Marshall) (?); Praon volucre (Haliday); Praon yomenae Takada (?).

Microlophium carnosum (Buckton): Aphidius ervi Haliday; Aphidius urticae Haliday.

Monellia caryella (Fitch): Trioxys pallidus (Haliday).

Myzaphis rosarum (Kaltenbach): Praon volucre (Haliday).

Myzocallis glandulosa Hille Ris Lambers: Aphidius myzocallidis Mescheloff \& Rosen.

Myzus ascalonicus Doncaster: Aphidius matricariae Haliday. 
Myzus beybienkoi (Narzikulov): Aphidius matricariae Haliday; Diaeretiella rapae (M'Intosh); Lysiphlebus fabarum (Marshall); Praon volucre (Haliday).

Myzus cerasi (F.): Aphidius matricariae Haliday; Ephedrus cerasicola Starý; Ephedrus persicae Froggatt; Ephedrus plagiator (Nees).

Myzus ornatus Laing: Aphidius matricariae Haliday.

Myzus persicae (Sulzer): Aphidius avenae Haliday; Aphidius colemani Viereck [- platensis Brèthes, in part]; Aphidius ervi Haliday; Aphidius matricariae Haliday; Aphidius platensis Brèthes; Aphidius ribis Haliday (?); Binodoxys angelicae (Haliday); Diaeretiella rapae (M'Intosh); Ephedrus cerasicola Starý; Ephedrus persicae Froggatt; Lysiphlebus confusus Tremblay \& Eady; Lysiphlebus fabarum (Marshall); Lysiphlebus testaceipes (Cresson); Praon necans Mackauer; Praon volucre (Haliday).

Nasonovia ribisnigri (Mosley): Aphidius hieraciorum Starý; Aphidius matricariae Haliday (?); Praon pubescens Starý.

Nearctaphis bakeri (Cowen): Aphidius smithi Sharma \& Subba Rao, Praon barbatum Mackauer.

Ovatus insitus (Walker): Aphidius matricariae Haliday; Monoctonus mali van Achterberg.

Ovatus mentharius (van der Goot): Aphidius colemani Viereck [- platensis Brèthes, in part].

Pemphigus spyrothecae Passerini: Monoctonia vesicarii Tremblay.

Periphyllus testudinaceus (Fernie): Aphidius setiger (Mackauer).

Periphyllus sp.: Aphidius setiger (Mackauer).

Phorodon humuli (Schrank): Aphidius matricariae Haliday; Aphidius platensis Brèthes; Ephedrus cerasicola Starý; Ephedrus persicae Froggatt; Lysiphlebus fabarum (Marshall); Praon volucre (Haliday).

Protaphis alexandrae (Nevsky): Lysiphlebus fabarum (Marshall).

Protaphis anthemidis (Börner): Lysiphlebus fabarum (Marshall).

Protaphis elongata (Nevsky): Lysiphlebus fabarum (Marshall).

Protaphis terricola (Rondani): Lysiphlebus fabarum (Marshall); Lysiphlebus desertorum Starý.

Protaphis spp.: Binodoxys acalephae (Marshall); Lysiphlebus confusus Tremblay \& Eady; Lysiphlebus desertorum Starý; Lysiphlebus fabarum (Marshall).

Pterochloroides persicae (Cholodkovsky): Pauesia antennata (Mukerji).

Pterocomma pilosum Buckton: Aphidius cingulatus Ruthe.

Pterocomma populeum (Kaltenbach): Aphidius cingulatus Ruthe.

Pterocomma sp.: Aphidius cingulatus Ruthe.

Rhopalomyzus sp.: Aphidius matricariae Haliday.

Rhopalosiphum maidis (Fitch): Aphidius avenae Haliday; Aphidius colemani Viereck [- platensis Brèthes, in part]; Aphidius matricariae Haliday; Aphidius platensis Brèthes; Aphidius rhopalosiphi De StefaniPerez; Aphidius uzbekistanicus Luzhetzki; Diaeretiella rapae (M'Intosh); Ephedrus persicae Froggatt; Ephedrus plagiator (Nees); Lysiphlebus fabarum (Marshall); Lysiphlebus testaceipes (Cresson); Praon gallicum Starý; Praon necans Mackauer; Praon volucre (Haliday).

Rhopalosiphum nymphaeae (L.): Aphidius colemani Viereck [- platensis Brèthes, in part]; Aphidius matricariae Haliday; Ephedrus cerasicola Starý; Lysiphlebus fabarum (Marshall); Praon necans Mackauer. 
Rhopalosiphum oxyacanthae (Schrank): Ephedrus plagiator (Nees).

Rhopalosiphum padi (L.): Aphidius colemani Viereck [- platensis Brèthes, in part]; Aphidius ervi Haliday; Aphidius matricariae Haliday; Aphidius platensis Brèthes; Aphidius rhopalosiphi De StefaniPerez; Aphidius uzbekistanicus Luzhetzki; Diaeretiella rapae (M'Intosh); Ephedrus persicae Froggatt; Ephedrus plagiator (Nees); Lysiphlebus fabarum (Marshall); Praon gallicum Starý; Praon necans Mackauer; Praon volucre (Haliday).

Saltusaphis scirpus Theobald: Diaeretiella rapae (M'Intosh); Lysiphlebus fabarum (Marshall).

Saltusaphis sp.: Lysiphlebus fabarum (Marshall).

Schizaphis graminum (Rondani): Aphidius colemani Viereck [- platensis Brèthes, in part]; Aphidius ervi Haliday; Aphidius matricariae Haliday; Aphidius platensis Brèthes; Aphidius rhopalosiphi De StefaniPerez; Aphidius uzbekistanicus Luzhetzki; Diaeretiella rapae (M'Intosh); Ephedrus persicae Froggatt; Ephedrus plagiator (Nees); Praon gallicum Starý; Praon necans Mackauer; Praon volucre (Haliday).

Schizaphis rosazevedoi Ilharco: Aphidius colemani Viereck [- platensis Brèthes, in part]; Aphidius matricariae Haliday.

Sipha elegans Del Guercio: Adialytus ambiguus (Haliday).

Sipha flava (Forbes): Adialytus ambiguus (Haliday).

Sipha maydis Passerini: Adialytus ambiguus (Haliday); Ephedrus persicae Froggatt (?).

Sipha sp.: Adialytus ambiguus (Haliday).

Sitobion avenae (F.): Aphidius colemani Viereck [- platensis Brèthes, in part]; Aphidius ervi Haliday; Aphidius matricariae Haliday; Aphidius platensis Brèthes; Aphidius rhopalosiphi De Stefani-Perez; Aphidius smithi Sharma \& Subba Rao (?); Aphidius uzbekistanicus Luzhetzki; Binodoxys angelicae (Haliday) (?); Diaeretiella rapae (M'Intosh); Ephedrus persicae Froggatt; Ephedrus plagiator (Nees); Lysiphlebus fabarum (Marshall) (?); Praon gallicum Starý; Praon necans Mackauer; Praon volucre (Haliday).

Sitobion fragariae (Walker): Aphidius colemani Viereck [- platensis Brèthes, in part]; Aphidius ervi Haliday; Aphidius matricariae Haliday; Aphidius rosae Haliday; Aphidius uzbekistanicus Luzhetzki; Praon volucre (Haliday).

Smynthurodes betae Westwood: Monoctonia pistaciaecola Starý.

Thelaxes confertae Börner: Adialytus thelaxis Starý.

Thelaxes suberi (Del Guercio): Adialytus thelaxis Starý; Trioxys quercicola Starý.

Thelaxes sp.: Adialytus thelaxis Starý.

Therioaphis khayami Remaudière: Trioxys complanatus Quilis.

Therioaphis riehmi (Börner): Praon exsoletum (Nees); Trioxys complanatus Quilis.

Therioaphis trifolii (Monell): Praon exsoletum (Nees); Trioxys complanatus Quilis.

Therioaphis sp.: Praon exsoletum (Nees).

Tinocallis nevskyi Remaudière, Quednau \& Heie: Praon flavinode (Haliday).

Tinocallis saltans (Nevsky): Betuloxys hortorum (Starý).

Titanosiphon neoartemisiae (Takahashi): Aphidius iranicus Rakhshani \& Starý; Trioxys pannonicus Starý; Trioxys tanaceticola Starý (?).

Tuberculatus (Tuberculoides) albosiphonatus Hille Ris Lambers: Praon flavinode (Haliday); Trioxys pallidus (Haliday). 
Tuberculatus (Tuberculoides) moerickei Hille Ris Lambers: Praon flavinode (Haliday); Trioxys pallidus (Haliday).

Tuberculatus sp.: Praon flavinode (Haliday); Trioxys pallidus (Haliday).

Uroleucon acroptilidis Kadyrbekov, Renxin \& Shao: Aphidius funebris Mackauer; Ephedrus niger Gautier, Bonnamour \& Gaumont; Praon unitum Mescheloff \& Rosen; Praon yomenae Takada.

Uroleucon aeneum (Hille Ris Lambers): Aphidius ervi Haliday (?); Aphidius funebris Mackauer; Praon volucre (Haliday); Praon yomenae Takada.

Uroleucon bielawskii (Szelegiewicz): Aphidius persicus Rakhshani \& Starý; Ephedrus niger Gautier, Bonnamour \& Gaumont.

Uroleucon carthami (Hille Ris Lambers): Aphidius uroleuci Mescheloff \& Rosen; Praon yomenae Takada; Aphidius persicus Rakhshani \& Starý.

Uroleucon chondrillae (Nevsky): Aphidius funebris Mackauer; Aphidius persicus Rakhshani \& Starý; Praon yomenae Takada.

Uroleucon cichorii (Koch): Aphidius funebris Mackauer; Ephedrus niger Gautier, Bonnamour \& Gaumont; Praon volucre (Haliday); Praon yomenae Takada.

Uroleucon compositae (Theobald): Aphidius matricariae Haliday (?); Aphidius funebris Mackauer; Aphidius persicus Rakhshani \& Starý; Ephedrus niger Gautier, Bonnamour \& Gaumont; Lysiphlebus fabarum (Marshall) (?); Lysiphlebus testaceipes (Cresson); Praon volucre (Haliday); Praon yomenae Takada.

Uroleucon erigeronense (Thomas): Aphidius funebris Mackauer; Ephedrus niger Gautier, Bonnamour \& Gaumont.

Uroleucon inulae (Ferrari): Aphidius uroleuci Mescheloff \& Rosen; Ephedrus niger Gautier, Bonnamour \& Gaumont.

Uroleucon inulicola (Hille Ris Lambers): Praon volucre (Haliday).

Uroleucon jaceae (L.): Aphidius funebris Mackauer; Aphidius uroleuci Mescheloff \& Rosen; Ephedrus niger Gautier, Bonnamour \& Gaumont; Lysiphlebus fabarum (Marshall) (?); Praon unitum Mescheloff \& Rosen; Praon volucre (Haliday); Praon yomenae Takada.

Uroleucon ochropus (Hille Ris Lambers): Aphidius persicus Rakhshani \& Starý.

Uroleucon picridis (Fabricius): Praon yomenae Takada.

Uroleucon sonchi (L.): Aphidius ervi Haliday (?); Aphidius matricariae Haliday (?); Aphidius funebris Mackauer; Aphidius persicus Rakhshani \& Starý; Aphidius uroleuci Mescheloff \& Rosen; Diaeretiella rapae (M'Intosh) (?); Ephedrus niger Gautier, Bonnamour \& Gaumont; Praon unitum Mescheloff \& Rosen; Praon volucre (Haliday); Praon yomenae Takada.

Uroleucon tortuosissimae Rezwani \& Lampel: Praon yomenae Takada.

Uroleucon sp.: Aphidius funebris Mackauer; Aphidius persicus Rakhshani \& Starý; Aphidius rosae Haliday (?); Ephedrus niger Gautier, Bonnamour \& Gaumont; Lysiphlebus confusus Tremblay \& Eady (?); Praon volucre (Haliday).

Wahlgreniella nervata (Gillette): Aphidius ervi Haliday; Aphidius matricariae Haliday; Praon rosaecola Starý.

Xerobion cinae (Nevsky): Lysiphlebus desertorum Starý. 


\section{Key to female Aphidiinae of the Middle East and North Africa}

1. Forewing with seven closed cells. Forewing 2RS present and well developed (Figs 118-125, 164)

- Forewing with four closed cells or fewer. Forewing 2RS absent (Figs 73-117, 126-163, 165175)

2. Antennae 18-segmented (Fig. 62). Ovipositor sheath deltoid shaped (Fig. 601)

.Toxares deltiger (Haliday)

- Antennae 13-segmented (Figs 31-35). Ovipositor sheath elongated (Figs 555-562)

3. Forewing $3 \mathrm{RSa}$ vein distinctly shorter than $2 \mathrm{RS}$ vein (Figs 119,124$)$. Petiole less than $1.5 \times$ as long as wide (Figs 419, 424)

- Forewing 3RSa vein subequal or distinctly longer than 2RS vein (Figs 118, 120-123, 125). Petiole more than $1.8 \times$ as long as wide (Figs $418,420-423,425$ )

4. Antenna slightly thickened at apex (Fig. 34). F1 4.00-4.50× as long as wide. Ovipositor sheath stout (Fig. 561)

Ephedrus persicae Froggatt

- Antenna distinctly thickened at the apex. F1 $4.50-4.80 \times$ as long as wide. Ovipositor sheath elongated (Fig. 556)

Ephedrus chaitophori Gärdenfors

5. Antennae thickened at apex, apical and praeapical segments broadly joined forming a club (Fig. 32). Ovipositor sheath stout and wide at base (Fig. 557)

Ephedrus helleni Mackauer

- Antennae filiform or only slightly thickened towards apex. Apical and praeapical segments normally joined, never forming a club (Figs 31, 33, 35) Ovipositor sheath medium sized (Figs 555, 559, 562) to elongated (Figs 558, 560), never widened at base

6. Flagellomere 1 very long, 1.4-1.5 $\times$ flagellomere 2. Forewing $r$ vein as long as or slightly longer than width of stigma (Fig. 121) Ephedrus lacertosus (Haliday)

- Flagellomer 1 stout, 1.3× flagellomere 2 or shorter (Figs 31, 33, 35). Forewing vein $r$ distinctly shorter than width of stigma (Figs 118, 122, 123, 125)

7. Ovipositor sheath considerably elongated with praeapical depression (Fig. 560)

Ephedrus niger Gautier, Bonnamour \& Gaumont

- Ovipositor sheath less elongated, without praeapical depression (Figs 555, 559, 562) 8

8. Petiole with strongly prominent central and less-developed lateral longitudinal carinae (Fig. 422). Flagellomere $13.00-3.50 \times$ as long as wide Ephedrus nacheri Quilis

- Petiole with less developed central and prominent lateral longitudineal carinae (Figs 418, 425). Flagellomere $13.80-4.20 \mathrm{x}$ as long as wide (Figs 31,35)

9. Flagellomere 1 yellow, $1.3-1.5 \times$ as long as flagellomere 2 bearing $1-2$ longitudinal placodes (Fig. 31)

Ephedrus cerasicola Starý

- Flagellomere 1 brown with narrow yellowish ring, 1.1-1.3 $\times$ as long as flagellomere 2 bearing 3-5 longitudinal placodes (Fig. 35)

Ephedrus plagiator (Nees)

10. Notauli complete (Figs 212, 245-263). Forewing RS+M vein present, sometimes colourless throughout or partially (Figs 109, 146-163)

- Notauli incomplete or absent (Figs 176-211, 213-220, 229-245, 265-271). Forewing RS+M vein absent (Figs 73-108, 110-117, 126-145, 165-175) 
11. Antennae 13-14-segmented (Fig. 25). Propodeum areolated (Fig. 308). Ovipositor sheath densely setose at apical half (Fig. 546) Areopraon lepelleyi (Waterson)

- Antennae 15-22(23)-segmented (Figs 47-61). Propodeum smooth (Figs 345-362). Ovipositor sheath sparsely setose (Figs 583-600)

12. Lateral lobes of mesonotum densely pubescent (Figs 250, 254, 257-259, 262)

- Lateral lobes of mesonotum with small (Figs 246, 248, 261) to large hairless areas (Figs 247, 249, $251-253,255,256,260,263)$

13. Forewing RS vein effaced, point-like (Fig. 157). Dorsal aspect of propodeum sparsely pubescent (Fig. 356) Praon orpheusi Kavallieratos, Athanassiou \& Tomanović

- Forewing RS never point-like and normally developed (Figs 150, 154, 158, 159, 162). Dorsal aspect of propodeum densely pubescent (Figs 349, 353, 357, 358, 361) 14

14. Flagellomere 1 yellow. Petiole elongated, $1.5-1.6 \times$ as long as wide at spiracle (Figs 450, 454). Antennae 19-22 (23)-segmented

- Flagellomere 1 brown, with small yellowish part at base. Petiole short, 1.20-1.35× as long as wide at spiracle (Figs 458, 459, 462). Antennae 16-18(19)-segmented 16

15. Antennae 19-segmented (Fig. 50). Flagellomere 1 yellow. Dorsal aspect of petiole with few sparse hairs along sides (Fig. 450). Dorsal outline of ovipositor sheath almost straight (Fig. 587)

Praon bicolor Mackauer

- Antenna 21-23-segmented (Fig. 54). Dorsal aspect of petiole with dense hairs along sides (Fig. 454). Dorsal outline of ovipositor sheath concave (Fig. 591) Praon longicorne Marshall

16. Dorsal aspect of petiole with sparse hairs along sides (Fig. 459). Stigma 1.9-2.3× as long as R1 vein (Fig. 159)

Praon rosaecola Starý

- Dorsal aspect of petiole with dense hairs along sides (Figs 458, 462). Stigma $1.25-1.65 \times$ as long as R1 vein (Figs 158, 162)

17. Antenna 16-17-segmented (Fig. 57). Ovipositor sheath rounded at apex (Fig. 595)

Praon pubescens Starý

- Antenna (17)18-19-segmented (Fig. 60). Ovipositor sheath sharply pointed at apex (Fig. 599) ....

Praon volucre (Haliday)

18. Lateral lobes of mesonotum with small hairless areas (Figs 246, 248, 261)

Lateral lobes of mesonotum with large hairless areas (Figs 247, 249, 251-253, 255, 256, 260, 263)

19. Forewing m-cu complete and coloured throughout (Fig. 146). Antenna 15-16-segmented (Fig. 47). Flagellomere 1 brown, yellowish at base, $4.0-4.5 \times$ as long as wide

Praon abjectum (Haliday)

- Forewing m-cu complete but colourless or effaced distally (Figs 148, 161). Antenna 20-21-segmented. Flagellomere 1 yellow with a dark ring at the apex and $0.5-0.6 \times$ as long as wide

20. Forewing vein m-cu colourless proximally and effaced distally (Fig. 161). Stigma $3.5 \times$ as long as wide and $1.5 \times$ as long as R1 vein. Antenna 21-segmented. Flagellomere $15.0 \times$ as long as wide. Petiole stout, subquadrate (Fig. 561) Praon uroleucon Tomanović \& Kavallieratos

- Forewing vein m-cu coloured on first third and colourless for remaning part (Fig. 148). Stigma 4.0× as long as wide and $1.8 \times$ as long as R1 vein. Antenna 20-segmented. Flagellomere 10.6 as long as 
wide. Petiole more elongated, $1.22 \times$ as long as wide at spiracles (Fig. 448)

Praon athenaeum Kavallieratos \& Lykouressis

21. Forewing m-cu complete and coloured throughout (Fig. 147). Flagellomere 1 brown, only yellowish at base

- Forewing m-cu coloureless throughout or partially or completely effaced (Figs 149, 151-153, 155, $156,160,163)$. Flagellomere 1 yellowish, sometimes darkened to brown at tip

22. Forewing m-cu vein developed, but partially colourless or thoroughly colourless (Figs 149, 151,



- $\quad$ Forewing m-cu vein effaced (Figs 153, 155). Stigma narrow, $3.6-4.2 \times$ as long as wide ............. 28

23. Antenna 20-21-segmented (Fig. 49). Ovipositor sheath considerably elongated (Fig. 586). Face and propodeum very densely pubescent (Fig. 348)

Praon barbatum Mackauer

- Antenna 15-19-segmented. Ovipositor sheath less elongated (Figs 588, 589, 593, 597, 600). Face and propodeum normally or sparsely pubescent (Figs 350, 351, 355, 359, 362)

24. Petiole less than $1.2 \times$ as long as wide. Dorsal aspect of petiole with a pair of triple hairs at posterior margin (Fig. 452). Ovipositor sheath stout with length/width ratio less than 2.4 (Fig. 589)

Praon flavinode (Haliday)

- Petiole more than $1.3 \times$ as long as wide. Dorsal aspect of petiole with sparse to dense hairs located between spircales (Figs 451, 456, 460, 463). Ovipositor sheath more elongated with length/width ratio more than 2.5 (Figs 588, 593, 597, 600)

25. Dorsal aspect of petiole almost hairless or with 4-5 short setae along sides (Fig. 451). Propodeum covered with few and scattered hairs (Fig. 350)

Praon exsoletum (Nees)

- Dorsal aspect of petiole with more than 10 short setae along each side (Figs 456, 460, 463).

Propodeum covered with several long hairs (Figs 355, 359, 362) 26

26. Antenna 18-19(20)-segmented (Fig. 61). Forewing cu vein coloured throughout (Fig. 163)

Praon yomenae Takada

- Antenna 16-17-segmented (Fig. 59). Forewing cu vein colourless throughout or coloured only prior to m-cu vein (Figs 156, 160) 27

27. Dorsal aspect of propodeum sparsely pubescent (Fig. 359). Flagellomere $16.0 \times$ as long as wide (Fig. 59). Mesosoma yellowish to yellow Praon unitum Mesheloff \& Rosen

- Dorsal aspect of propodeum densely pubescent (Fig. 355). Flagellomere $15.5 \times$ as long as wide. Mesosoma dark brown Praon nonveilleri Tomanović \& Kavallieratos

28. Flagellomeres 1 and 2 yellow. Stigma 2.0× as long as R1 vein (Fig. 153). Dorsal aspect of propodeum covered with normal hairs (Fig. 352)

Praon gallicum Starý

- Flagellomere 1 yellow with brown apice, flagellomere 1 brown. Stigma as long as R1 vein (Fig. 155). Dorsal aspect of propodeum covered with very long hairs (Fig. 354) .......Praon necans Mackauer

29. Forewing $1 \mathrm{RS}$ vein long, reaching R1 vein at tip of wing margin (Fig. 73). Petiole very short, $0.8 \times$ as long as wide at spiracles (Fig. 374). Ovipositor sheath dagger shaped (Fig. 510). Eyes considerably reduced in size Aclitus obscuripennis Förster

- Forewing 1RS short, never reaching R1 vein and wing margin (Figs 74-108, 110-117, 126-145, 165-175). Petiole always longer than width at spiracles (Figs 375-408, 410-417, 426-445, 465475). Ovipositor sheath of different shapes (Figs 511-545, 547-554, 563-582, 602-612). Eyes normally developed 
30. Terminal metasomal sternum with a pair of prongs (Figs 547-552, 602-612) .......................... 31

- Terminal metasomal sternum without prongs (Figs 511-545, 553, 554, 563-582) .................... 47

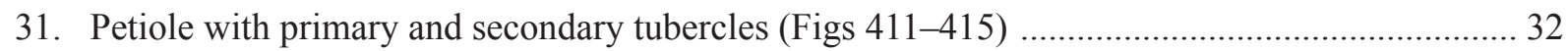

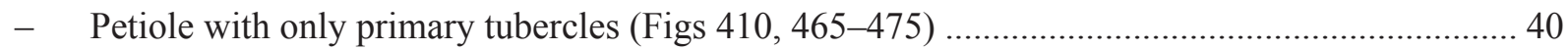

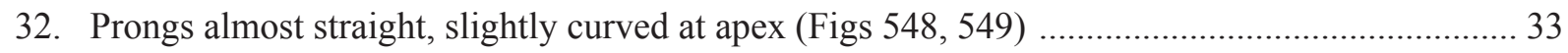

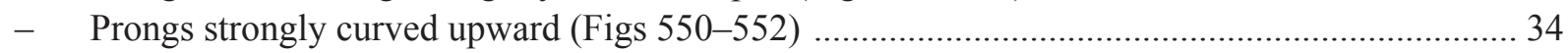

33. Distance between primary and secondary tubercles less than width at spiracles (Fig. 411). Stigma 2.1-2.3× as long as wide and 1.9-2.1× as long as R1 vein (Fig. 111). Ovipositor sheath subquadrate at base (Fig. 548). Metasoma dark brown

Binodoxys acalephae (Marshall)

- Distance between primary and secondary tubercles more than width at spiracles (Fig. 412). Stigma 2.6-2.8× as long as wide and 1.4-1.6× as long as R1 vein (Fig. 112). Ovipositor sheath rounded at base (Fig. 549). Petiole and last metasomal segments yellowish brown

Binodoxys angelicae (Haliday)

34. Dorsal aspect of prongs with 7-8 long hairs (Fig. 551)

Binodoxys centaureae (Haliday)

- Dorsal aspect of prongs with 2-3 long hairs (Figs 550, 552) ....

35. Antenna 10-segmented. (Fig. 28). Propodeum smooth with only posterolateral carinae developed at base (Fig. 312). Primary and secondary tubercles almost fused, distance between them less than width of petiole at spiracles (Fig. 413)

Binodoxys brevicornis (Haliday)

- Antenna 11-segmented. (Fig. 29). Propodeum carinated with well developed and complete central areola (Fig. 314). Primary and secondary tubercles distinctly separated, distance between them more than width at spiracles (Fig. 415)

Binodoxys heraclei (Haliday)

36. Apical portion of prongs differentiated, bearing several stout basally dilated bristles (Fig. 547) ....

Betuloxys horturom (Starý)

- Apical portion of prongs tubular with 1-2 bristles of various shapes (simple, ovoid or uniformly



37. R1 vein reduced, equal to or shorter than half of stigma (Figs 156, 169, 172, 175) .................. 38 R1 vein well developed, distinctly longer than half of stigma (Figs 166, 167, 168, 170, 171, 173, 174)

38. Prongs very short and straight with a pair of ovoid-shaped bristles at apex (Fig. 612). R1 vein $0.45-0.5 \times$ as long stigma (Fig. 175)

Trioxys tanaceticola Starý

- Prongs long and distinctly curved upward with a pair of simple or uniformly dilated bristles at apex (Figs $602,606,609)$. R1 vein less than $0.35 \times$ as long stigma (Fig. 165, 169, 172)

39. Prongs with 3-4 long setae at dorsal surface and a pair of simple bristles at apex (Fig. 606). Maxillary and labial palps with 3 and 1 palpomeres, respectively .. Trioxys metacarpalis Rakhshani \& Starý

- Prongs with more than 6-7 long setae at dorsal surface and a pair of uniformly dilated bristles (Figs 602, 609). Maxillary and labial palps with 4 and 2 palpomeres, respectively

40. Antenna 11-segmented (Fig. 70). Petiole short, less than $1.8 \times$ as long as wide at spiracles (Fig. 472)

- Antenna 12-segmented (Fig. 63). Petiole elongated, more than $2.2 \times$ as long as wide at spiracles (Fig. 465) 
41. Apex of prongs with single claw-shaped bristle (Figs 604, 605, 607, 608, 611)

Apex of prongs with a pair of simple or uniformly dilated bristles (Figs 603, 610)

42. Prongs distinctly curved upward (Fig. 605)

Trioxys curvicaudus Mackauer

- Prongs straight or very slightly curved (Figs 604, 607, 608, 611) .

43. Prongs without any long setae at dorsal surface (Fig. 611)

Trioxys quercicola Starý

- Prongs with 1-4 long setae at dorsal surface (Figs 604, 607, 608) 44

44. Prongs with 1-2 long setae at dorsal surface (Fig. 607) ........ Trioxys moshei Mescheloff \& Rosen

- Prongs with 3-4 long setae at dorsal surface (Figs 604, 608) 45

45. Ovipositor sheath short, $2.10-2.20 \times$ as long as wide at base (Fig. 604). R1 vein $0.60-0.80 \times$ as long stigma (Fig. 167)

Trioxys complanatus Quilis

- Ovipositor sheath elongated, $2.90-3.10 \times$ as long as wide at base (Fig. 608). R1 vein $0.5 \times$ as long stigma (Fig. 171)

Trioxys pallidus (Haliday)

46. Propodeum areolated with well developed anterolateral and central carinae (Fig. 365). Prongs almost straight with 6-8 long setae at dorsal surface and a pair of uniformly dilated bristles at apex (Fig. 603)

Trioxys cirsii (Curtis)

- Propodeum smooth having short posterolateral carinae at base. Prongs upcurved apically with 4-5 long setae at dorsal surface and a pair of simple bristles at apex (Fig. 610) ..Trioxys pappi Takada

47. Propodeum reticulated and irregularly areolated (Figs 332, 333). Petiole short and subquadrate, $1.0-1.2 \times$ as long as wide at spiracles (Figs 433, 434). Antenna monilliform (Figs 43, 44) ........ 48

- Propodeum smooth (Figs 273-276, 326-331) or regularly areoleted (Figs 277-307, 315, 316, 325, 334-344). Petiole elongated, more than $1.5 \times$ as long as wide at spiracles (Figs 375-408, 416, 417, 426-432, 435-445). Antenna filiform (Figs 2-24, 30, 36-42, 45, 46)

48. Antenna 12-13-segmented. F1 2.6-2.7× as long as wide. F1 and F2 with 1-2 and 5-6 longitudinal placodes, respectively (Fig. 43). Ovipositor sheath triangular, curved downwards, narrowed to the apex (Fig. 570)

Monoctonia pistaciaecola Starý

- Antenna 16 (17)-segmented. F1 1.75-2.00× as long as wide. F1 and F2 with 0 and 1 longitudinal placodes, respectively (Fig. 44). Ovipositor sheath quadrangular at base, sharply narrowed ventrally, truncated at tip (Fig. 571) Monoctonia vesicarii Tremblay

49. Ovipositor sheath ploughshare shaped (Figs 572, 573)

Ovipositor sheath of different shapes, cup shaped (Fig. 563), subovoid (Figs 515-545, 553, 574.

576. 580, 581), triangular (Figs 511-514, 564-569), quadrangular (Fig. 554), spatulated (Fig. 578), slender (Fig. 579), acincaciform (Figs 575, 577, 582)

50. Forewing $\mathrm{r} \& \mathrm{RS}, \mathrm{M}+\mathrm{m}-\mathrm{cu}$ and $\mathrm{r}-\mathrm{m}$ veins well developed and visible (Fig. 135). Petiole elongated, 2.0-2.2 $\times$ as long as wide at spiracles (Fig. 435) Monoctonus crepidis (Haliday)

- Forewing $\mathrm{r} \& \mathrm{RS}, \mathrm{M}+\mathrm{m}-\mathrm{cu}$ and $\mathrm{r}-\mathrm{m}$ veins effaced (Fig. 136). Petiole short, 1.6-1.8× as long as wide at spiracles (Fig. 436) Monoctonus mali van Achterberg

51. Forewing $r \&$ RS vein extending over tip of R1 vein, reaching the outer border of forewing (Fig. 126). Dorsal aspect of petiole with a pair of strong carinae, diverging backward (Fig. 426). Ovipositor sheath elongated-cup shaped, strongly downcurved (Fig. 563)

Lipolexis gracilis Förster

- Forewing r \& RS vein reaching the end of R1 vein in maximum length (Figs 74-108, 116, 117, 127-132, 137-145). Dorsal aspect of petiole smooth, slightly swollen or with straight central keel 
(Figs 375-408, 416, 417, 427-432, 437-445). Ovipositor sheath of different shapes (Figs 511-545, $553-554,564-569,574-582)$

52. Propodeum with very narrow, small, central areola (Figs 277-307, 315) or smooth (Figs 273-276, 326-331)

- Propodeum with wide central pentagonal areola $(336-342,344)$ or only with anterolateral and central carinae (Figs 316, 343)

53. Dorsal aspect of propodeum carinated with complete or incomplete central areola (Figs 277-307, 315). Ovipositor sheath truncated at tip (Figs 515-545, 553)

- Dorsal aspect of propodeum smooth or with two divergent carinae at lower part (Figs 273-276, 326-331). Ovipositor sheath pointed at tip (Figs 511-514, 564-569)

54. Forewing $\mathrm{r}-\mathrm{m}$ and $\mathrm{M}+\mathrm{m}-\mathrm{cu}$ veins absent (Fig. 116)

Diaeretiella rapae (M'Intosh)

- Forewing $\mathrm{r}-\mathrm{m}$ vein present, $\mathrm{M}+\mathrm{m}$-cu complete (Figs 78, 80-109) or reduced anteriorly (Fig. 79) ...55

55. Anterolateral area of petiole rugose (Figs 483, 486) 56 Anterolateral area of petiole costate (Figs 478, 481, 388, 496), or costulate (Figs 476-477, 479$480,482,484-485,487-495,497-499)$

56. Stigma 3.8-4.3× as long as wide (Fig. 92). Anterodorsal area of ovipositor sheath strongly elevated leading to a clearly concave dorsal outline (Fig. 529)

Aphidius microlophii Pennachio \& Tremblay

- Stigma 3.4-3.9× as long as wide (Fig. 87). Antero-dorsal area of ovipositor sheath moderately elevated leading to a slightly concave dorsal outline (Fig. 524) Aphidius ervi Haliday

57. Anterolateral area of petiole costate (Figs 478, 481, 388, 496) 58 Anterolateral area of petiole costulate (Figs 476-477, 479-480, 482, 484-485, 487-495, 497499)

58. Labial palps with three palpomeres. Length of stigma 1.6-2.0× R1 vein (Figs 81, 105). Antenna (16)17-18-segmented

- Labial palps with two palpomeres. Length of stigma 1.1-1.2× R1 vein (Figs 84, 96). Antenna (14)15-16-segmented

59. Petiole with swollen anterodorsal area (Fig. 405). General body color yellowish brown

Aphidius transcaspicus Telenga

- Petiole with deep anterodorsal area (Fig. 382). General body color dark brown to black

Aphidius avenae Haliday

60. Anterolateral area of petiole smooth with 3-4 blunt costae (Fig. 385)

Aphidius colemani Viereck

- Anterolateral area of petiole strongly curved with 5-6 sharp costae (Fig. 395)

Aphidius platensis Brèthes

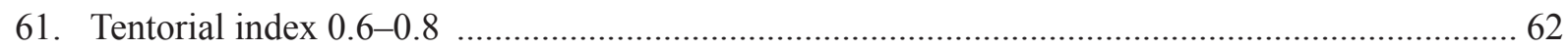

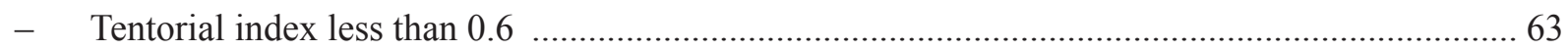

62. Antenna (18)19-20-segmented. Flagellomeres 1 and 2 with 2-5 and 3-7 longitudinal placodes, respectively (Fig. 8). Petiole 3.00-3.50× as long as wide at spiracles (Fig. 384) 
- Antenna13-14-segmented.Flagellomeres 1 and2 with1-3 and3-5longitudinal placodes, respectively. Petiole $2.50-3.00 \times$ as long as wide at spiracles (Fig. 401) Aphidius setiger (Mackauer)

63. Maxillary palps with 3 palpomeres, if one palp is with 4 palpomeres then the last palpomere bears trace of two segments

- Maxillary palps clearly 4-segmented 67

64. Labial palps with 2 palpomeres. Propodeum with complete central areola (Figs 290, 292) ........ 65

- $\quad$ Labial palps with 1 palpomere. Propodeum with incomplete central areola (Figs 278, 289) ...... 66

65. Stigma $4.5 \times$ as long as wide (Fig. 93). Clypeus with 6 scattered long setae. Flagellomere 1 basally yellow becoming brown midway to apex Aphidius myzocallidis Mescheloff \& Rosen

- Stigma 3.3-3.8× as long as wide (Fig. 91). Clypeus with 10-12 long setae. Flagellomere 1 yellowish brown with narrow apical dark ring

Aphidius matricariae Haliday

66. Dorsal aspect of petiole with a pair of convergent grooves (Fig. 380). Forewing M+m-cu vein incomplete, stigma $2.0 \times$ as long R1 vein (Fig. 79) Aphidius arvensis (Starý)

- Dorsal aspect of petiole smooth with fine rugosities (Fig. 391). Forewing M+m-cu vein complete, stigma equal or slightly longer than R1 vein (Fig. 90) Aphidius iranicus Rakhshani \& Starý

67. Labial palps with two palpomeres 68

- Labial palps with three palpomeres 75

68. Stigma narrowly triangular, $4.0-4.5 \times$ as long as wide, subequal to R1 vein $(1.0-1.2 \times$ as long R1 vein) (Figs 86, 98). Antenna 14-15-segmented 69

- Stigma widely triangular, 2.8 $-3.8 \times$ as long as wide, distinctly longer than R1 vein $(1.3-7.52 \times$ as long as R1 vein) (Figs 78, 80, 94, 96, 104, 106). Antenna (15)16-17-segmented 70

69. Apical flagellomere of antenna distinctly thickened. Flagellomere $12.6-3.0 \times$ as long as wide. Upper areola of propodeum with 2-4 long setae (Fig. 284). Dorsal outline of ovipositor sheath concave (Fig. 523)

Aphidius eglanteriae Haliday

- Apical flagellomere of antenna not thickened. Flagellomere $13.1-3.4 \times$ as long as wide. Upper areola of propodeum with 5-7 long setae (Fig. 297). Dorsal outline of ovipositor sheath smooth (Fig. 535)

Aphidius ribis Haliday

70. R1 vein very short, $0.15-0.20 \times$ as long as stigma (Fig. 96) Aphidius popovi Starý

- $\quad$ R1 vein short, $0.45-0.75 \times$ as long as stigma (Figs $78,80,94,104,106)$.................................. 71

71. Dorsal aspect of petiole smooth with fine rugosities (Figs 379, 381, 404) ............................... 72

_ Dorsal aspect of petiole with moderately prominent central carina (Figs 394, 406) ................... 74

72. Flagellomere 1 elongated, $4.06-4.33 \times$ as long as wide (Fig. 21)

Aphidius stigmaticus Rakhshani \& Tomanović

- Flagellomere 1 short, 3.00-3.50× as long as wide (Figs 6, 13) 73

73. Antenna 15-segmented. Petiole 2.00-2.50× as long as wide at spiracles (Fig. 381)

Aphidius asteris Haliday

- Antenna 16-17-segmented (Fig. 6). Petiole 3.00-3.50× as long as wide at spiracles (Fig. 379) ..... 
74. Propodeum with closed central areola. Upper areola of propodeum with 5-8 long setae (Fig. 293). Antenna 16-17-segmented Aphidius persicus Rakhshani \& Starý

- Propodeum with incomplete closed central areola by weak and broken carinae. Upper areola of propodeum with 3 long setae (Fig. 305). Antenna 15-16-segmented (Fig. 13)

Aphidius uroleuci Mescheloff \& Rosen

75. Antenna 13-14-segmented (Fig. 18) Aphidius salicis Haliday

- Antenna with more than 14 segments 76

76. Ovipositor sheath elongate and strongly prominent (Fig. 525) Aphidius funebris Mackauer

- Ovipositor sheath broad (Figs 519, 522, 526, 534, 536, 539-540, 544-545) 77

77. Antenna 15-18-segmented 78

- Antenna 19-20-segmented

78. R1 vein $0.50-0.55 \times$ as long as stigma (Fig. 99) Aphidius rosae Haliday

- $\quad$ R1 vein more than $0.75 \times$ as long as stigma (Figs $87,97,103,108)$ 79

79. Antenna 15-16-segmeneted 80

- Antenna 16-17-segmeneted 81

80. Stigma 3.5-4.0× as long as wide (Fig. 89). Flagellomere $12.5-3.0 \times$ as long as wide. Petiole, 3.0-3.5 $\times$ as long as wide at spiracles (Fig. 390) Aphidius hieraciorum Starý

- Stigma 3.0-3.5 $\times$ as long as wide (Fig. 103). Flagellomere $13.0-3.5 \times$ as long as wide. Petiole $2.5-3.0 \times$ as long as wide at spiracles (Fig. 403) Aphidius sonchi Marshall

81. Stigma elongately triangular, 3.30-3.90× as long as wide (Fig. 97). Dorsal aspect of petiole with strong rugosities and unclear central carina, anterolateral aspect with 10-12 straight costulae extended over posterior half (Fig. 490) Aphidius rhopalosiphi De Stefani-Perez

- Stigma widely triangular, 2.80-3.10× as long as wide (Fig. 108). Dorsal aspect of petiole with fine rugosities and prominent central carina, anterolateral area with aspect with 10-14 curved costulae, limited to the anterior half (Fig. 499) Aphidius uzbekistanicus Luzhetzki

82. Stigma 1.5-2.2× as long as R1 vein (Fig. 85). Antenna 20-21-segmented (Fig. 9) Aphidius eadyi Starý, Gonzáles \& Hall

- Stigma 1.1-1.3× as long as R1 vein (Figs 82, 102, 107). Antenna 19-20-segmented 83

83. Forewing $\mathrm{R} 1$ vein $1.9-2.2 \times$ as long as $\mathrm{r}$ vein (Fig. 107) Aphidius urticae Haliday

- Forewing R1 vein $1.4-1.6 \times$ as long as $r$ vein (Figs 82, 102) 84

84. Propodeum with wide central pentagonal areola (Fig. 281). Petiole $3.0-3.2 \times$ as long as wide at spiracles, its posterior part strongly rugose (Fig. 383). Anterolateral area of petiole with 9-10 irregular curved costulae (Fig. 479) Aphidius banksae Kittel

- Propodeum with narrow and small central pentagonal areola (Fig. 301). Petiole 2.4-2.8× as long as wide at spiracles, its posterior part mainly smooth (Fig. 402). Anterolateral area of petiole with 4-6 almost straight costulae (Fig. 494) Aphidius smithi Sharma \& Subba Rao

85. Forewing $\mathrm{M}+\mathrm{m}-\mathrm{cu}$ and $\mathrm{r}-\mathrm{m}$ veins absent (Fig. 117). Ovipositor sheath stout, subquadrate (Fig. 554). Notauli absent (Fig. 220). Antenna 15-16-segmented Diaeretus leucopterus (Haliday) 
- $\quad$ Forewing $\mathrm{M}+\mathrm{m}-\mathrm{cu}$ and $\mathrm{r}-\mathrm{m}$ veins present (Figs 137-145). Ovipositor sheath elongated in different shape (Figs 574-582). Notauli developed in anterior part of mesonotum (Figs 240-245). Antenna with more than 17 segments 86

86. Stigma narrow, its width distinctly less than $r$ vein (Fig. 141). Ovipositor sheath with lateral spatula (Fig. 578)

- Stigma wide, its width equal or distinctly more than $r$ vein (Figs 137-140, 142-145). Ovipositor sheath without lateral spatula (Figs 574-577, 579-582)

87. Ovipositor sheath elongated, $4.0-5.0 \times$ as long as wide at base (Figs 579,582 )

88. Antenna 21-segmented. Ovipositor sheath slender, upcurved, apically rounded (Fig. 579). Propodeum densly setose, with small central pentagonal areola (Fig. 341). Petiole elongated, $3.4-$ $3.6 \times$ as long as wide at spircales (Fig. 442)

Pauesia picta (Haliday)

- Antenna 16-17-segmented. Ovipositor sheath narrow and long, almost straight, apically pointed (Fig. 582). Propodeum sparsely setose, with large central pentagonal areola (Fig. 344). Petiole short, $2.8-3.2 \times$ as long as wide at spircales (Fig. 445)

Pauesia unilachni (Gahan)

89. Posterolateral carinae of propodeum absent, only the strong anterolateral carinae and a weak central carina present (Fig. 343)

Pauesia silana Tremblay

- Posterolateral carinae of propodeum present, defining the central areola (Figs 336-339, 342)... 90

90. Antenna 17-19-segmented. Propodeum with small central areola (Figs 337, 339). Ovipositor sheath strongly curved upwards, pointed apically (Figs 575, 577)

- Antenna 20-22-segmented. Propodeum with large central areola (Figs 336, 338, 342). Ovipositor sheath very slightly curved upward, apically rounded or truncated (Figs 574, 576, 580)

91. Antenna 18-19-segmented. Propodeum with complete and straight posterolateral carinae (Fig. 337). Stigma 3.2-3.5× as long as wide (Fig. 138). Petiole with spiracales located at half of the segment, slightly widened posteriorly, 3.2-3.3× as long as wide at spiracles (Fig. 438) Pauesia anatolica Michelena, Assael \& Mendel

- Antenna 17-segmented. Propodeum with incomplete and irregular posterolateral carinae (Fig. 339). Stigma 2.5-2.8× as long as wide (Fig. 140). Petiole with spiracles located at anterior one third of the segment, parallel sided, $3.5 \times$ as long as wide at spiracles (Fig. 440)

Pauesia cedrobii Starý \& Leclant

92. Propodeum densly setose, with irregular carinae (Fig. 338). Petiole parallel sided (Fig. 439). Ovipositor sheath truncated apically (Fig. 562)

Pauesia antennata (Mukerji)

- Propodeum sparsely setose, with regular carinae (Figs 336, 342). Petiole with prominent spiracular tubercles, distinctly widened to the base (Fig. 437, 443). Ovipositor sheath truncated apically (Figs 574, 580)

93. Stigma uniformly brown. Propodeum with less concave central areola (Fig. 336)

Pauesia abietis (Marshall)

- Stigma brown, yellowish at base. Propodeum with very concave central areola (Fig. 342)

Pauesia pini (Haliday) 




- $\quad$ Forewing with incomplete $\mathrm{M}+\mathrm{m}-\mathrm{cu}$ vein, $\mathrm{r}$ vein distinct (Figs 127-131) .................................... 98

95. Ovipositor sheath considerably elongated, lengh/width ratio 2.80-3.20 (Fig. 511)

Adialytus ambiguus (Haliday)

- Ovipositor sheath stout, lengh/width ratio 2.20-2.70 (Figs 512-514) 96

96. Flagellar segments (Fig. 4) subquadrate, slightly longer than their maximum width, length/width ratio 1.50-1.60. Flagellar segments and hind femur covered with long and prevalently erected setae (Fig. 502). Ovipositor sheath sharply angular (Fig. 513) Adialytus thelaxis (Starý)

- Flagellar segments (Figs 3, 5) cylindrical, considerably longer than their maximum width, length/ width ratio 2.00-2.90. Flagellar segments and hind femur covered with semi-erected (Fig. 501) or adpressed (Fig. 503) setae. Ovipositor sheath roundly angular (Figs 512, 514)

97. Petiole elongated, 2.20-2.40× as long as wide at spiracles (Fig. 376). Flagellar segments (Fig. 3) covered with prevalently semi-erected setae equal to segment diameter. Flagellomere 1 with 3-4 longitudinal placodes. Hind femur covered with prevalently semi-erected setae (Fig. 501)

Adialytus salicaphis (Fitch)

- Petiole short, 1.90-2.10× as long as wide at spiracles (Fig. 378). Flagellar segments (Fig. 5) covered with adpressed setae distinctly shorter than segment diameter. Flagellomere 1 with $0-1$ longitudinal placode. Hind femur covered with short adpressed setae (Fig. 503)

Adialytus veronicaecola (Starý)

98. Stigma equal or distinctly longer than R1 vein. (Figs 129, 131, 132). Petiole narrowly triangular (Figs 429, 431, 432). Labial palps with 2 palpomeres

- Stigma distinctly shorter than R1 vein, reaching outer margin of wing (Figs 127, 128, 130). Petiole widely triangular (Fig. 427, 428, 430). Labial palps with 1 palpomere 101

99. Petiole wide at base, $1.0-1.3 \times$ as long as wide at base (Fig. 429). Hind femur with semi-erected setae (Fig. 506)

Lysiphlebus desertorum Starý

- Petiole more elongate, 1.5-2.0× as long as wide at base (Figs 431, 432). Hind femur with appressed setae (Figs 508, 509) 100

100. Petiole $1.5-1.7 \times$ as long as wide at base (Fig. 431)

- Petiole 1.7-2.0× as long as wide at base (Fig. 432)

Lysiphlebus fritzmuelleri Mackauer Lysiphlebus testaceipes (Cresson)

101. Forewing marginal setae longer than those on the surface (Fig. 128)

Lysiphlebus confusus Tremblay \& Eady

- Forewing marginal setae as long as those on the surface (Figs 127, 129) 102

102. Hind femur with appressed setae (Fig. 507). Stigma 2.8-3.4× as long as wide (Fig. 130)

Lysiphlebus fabarum (Marshall)

- $\quad$ Hind femur with semi-erected setae (Fig. 504). Stigma 3.2-4.0× as long as wide (Fig. 127) ... 103

103. Stigma 3.2-3.6× as long as wide (Fig. 127). Flagellomere 1 usually without or exceptionally with 1 longitudinal placode (Fig. 37) Lysiphlebus cardui (Marshall)

- Stigma 3.8-4.0× as long as wide. Flagellomere 1 with 1-2 longitudinal placodes 
Table 1. Numbers of recorded genera and species of Aphidiinae in different countries of the Middle East and North Africa.

\begin{tabular}{llll}
\hline Countries & Area $(\mathbf{k m 2})$ & $\begin{array}{l}\text { Number } \\
\text { genera }\end{array}$ & $\begin{array}{l}\text { of } \\
\text { species }\end{array}$ \\
\hline Algeria & $2,381,741$ & 7 & 29 \\
Bahrain & 765.3 & - & - \\
Djibeuti & 23,200 & - & - \\
Egypt & $1,001,449$ & 8 & 22 \\
Eritrea & 117,598 & - & - \\
Iran & $1,648,000$ & 16 & 75 \\
Iraq & 437,072 & 10 & 28 \\
Israel & 20,770 & 11 & 38 \\
Jordan & 89,342 & 3 & 3 \\
Kuwait & 17,820 & - & - \\
Lebanon & 10,452 & 7 & 13 \\
Libya & $1,759,540$ & 2 & 4 \\
Morocco & 446,550 & 8 & 19 \\
Oman & 309,501 & - & - \\
Palestine & 6,220 & 3 & 4 \\
Qatar & 11,571 & - & - \\
Saudi Arabia & $2,150,000$ & 4 & 8 \\
Somalia & 637,657 & - & - \\
Sudan & $1,886,068$ & - & - \\
Syria & 185,180 & 4 & 3 \\
Tunisia & 163,610 & 7 & 16 \\
Turkey & 783,562 & 13 & 57 \\
UAE & 83,600 & 5 & 9 \\
Yemen & 528,076 & 7 & 11 \\
\hline
\end{tabular}

\section{Discussion}

The overall number of the recorded species from the Middle East and North Africa is less than one fifth of the total known Aphidiinae species, worldwide. Among the 24 counties of the Middle East and North Africa (Table 1), there are still no published records on aphid parasitoids from eight countries (Bahrain, Djibeuti, Eritrea, Kuwait, Oman, Qatar, Somalia, Sudan) (Fig. 613). Except on a few occasions, the explored areas of the Middle East and Noth Africa were mostly limited to the agricultural landscapes and nearby areas (Abou-Fakhr \& Kawar 1998; Havelka et al. 2011; Ahmad \& Bakr 2013; Starý et al. 2013; Tomanović et al. 2014). With a diverse range of habitats explored, Iran and Turkey are the most investigated regions of the Middle East and North Africa, in regards to Aphidiinae. For example, Barahoei et al. (2014) recorded 78 species of aphid parasitoids, belonging to 17 genera, from Iran (including some misidentified genera and species). However, the last up-to-date of Aphidiinae of Iran (Farahani et al. 2016) includes 73 species belonging to 15 genera. Two genera, Toxares Haliday and Euaphidius Mackauer, are not listed in Farahani et al. (2016) since the former is considered as a misidentification, while species of the latter are classified in Aphidius Nees on the basis of molecular and morphological analyses (Milošević et al. 2015). Incorporating a missing record, Lysiphlebus cardui (Alikhani et al. 
2013) and the newly recorded species (Kargarian et al. 2016; Farahani et al. 2017), the number of Aphidiinae species in Iran reaches 76. Among the recorded aphidiines of Iran, four species are recorded only from Iran. Twenty-four species are only recorded from Iran in the Middle East and North Africa regions, but are also recorded from elsewhere in the world.

On the basis of biogeographical complexity, Aphidiinae of the Middle East and North Africa can be categorized in three groups consisting of endemic rare, endemic widely distributed, or invasive species. The first group is represented by few species (i.e., Aphidius myzocallidis, Aphidius uroleuci, Trioxys moshei and Trioxys quercicola), which have not been collected since their original description. A large group of species includes native elements of the Western Palaearctic region that are irregularly distributed in the Middle East and North Africa. The well known invasive species Lysiphlebus testaceipes which is probably native to South America (Starý et al. 2014) is now widely distributed in Europe (Kavallieratos et al. 2001, 2004, 2005, 2010, 2013; Žikić et al. 2015; Kavallieratos et al. 2016; Tomanović et al. 2018) and North African countries (Laamari \& Coeur d' Acier 2010; Havelka et al. 2011; BoukhrisBouhachem 2011; Ben Halima 2011; Mitrović et al. 2013; Tomanović et al. 2018). This species has even been recorded in Iran (Rakhshani et al. 2005a) and Turkey (Uysal et al. 2004; Yoldaş et al. 2011; Satar et al. 2014). Some other species have been recorded occasionally and can be classified as rare taxa of the Middle East and North Africa, although they are widely distributed in Europe (i.e., Aphidius eglanteriae, Binodoxys centaureae, Diaeretus leucopterus, Ephedrus lacertosus, Ephedrus nacheri, Lysiphlebus cardui, Pauesia spp., Praon flavinode, Praon longicorne and Praon pubescens). Seven species (i.e., Aphidius eglanteriae, Aphidius microlophii, Lysiphlebus fritzmuelleri, Pauesia anatolica, Praon athenaeum, Praon nonveilleri and Praon uroleucon) are exclusively recorded in the western part of Turkey (Barjadze et al. 2010; Michelena et al. 2005; Akar \& Erdoğan 2017) and possibly could not be considered as elements of the Middle East area. Binodoxys centaureae, Pauesia cedrobii and Pauesia silana are members of the Mediterranean region, which are only recorded in North Africa (Starý 1976; Starý \& Leclant 1977; Laamari et al. 2012; Benhamacha et al. 2017). Few species of the genus Pauesia have been recorded in association with Cinarinae aphids in Middle Eastern countries, i.e., Algeria - Pauesia silana (Benhamacha et al. 2017); Iran - Pauesia antennata (Rakhshani et al. 2005b) and Pauesia hazratbalensis (Starý et al. 2005); Iraq - Pauesia antennata (Starý \& Kaddou 1971); Morocco - Pauesia cedrobii (Starý \& Leclant 1977; Fabre \& Rabasse 1987); Saudi Arabia Pauesia sp. (Ahmad \& Bakr 2013; Starý et al. 2013); Tunisia (Mdellel et al. 2015) and Yemen (Cross \& Poswal 1996) - Pauesia antennata. Furthermore, six species have been recorded from Turkey (Pauesia abietis - Schimitschek 1944; Starý 1976; Pauesia unilachni - Düzgüneş et al. 1982; Pauesia picta Aslan et al. 2004, Uysal et al. 2004; Pauesia anatolica - Michelena et al. 2005) and Israel (Pauesia pini - Bodenheimer \& Swirski 1957; Avidov \& Harpaz 1969; Starý 1976; Pauesia silana - Mescheloff \& Rosen 1990b; Pauesia anatolica - Michelena et al. 2005) as representative species of the Mediterranean region.

Recent evidence (Ghaliow et al. 2018) indicated that Aphidius banksae [former Aphidius staryi (Das \& Chakrabarti 1990), synonymy by Kittel (2016)], which is described from Israel and Turkey, occurs in Europe and it is widely distributed from Greece to Great Britain. Aphidius colemani is a biological control agent distributed in Europe and the Mediterranean region. Recent molecular analysis revealed the existence of another related species, Aphidius platensis (Tomanović et al. 2014) in Iran, which is considered to be the same species as in Chile (South America). Our new material from Saudi Arabia also confirms the broader distribution of A. platensis in the Middle East, while recent records of this species in India (Lokeshwari et al. 2016) suggest an even further expanded area of distribution.

Among the 743 parasitoid-aphid associations from Middle Eastern and North Africa, 52 records were doubtful and need further investigation. They may correspond to data originated from mixed aphid colonies and/or misidentifications. For example, the parasitoid complex of Urolecuon aphids is reviewed 
by Rakhshani et al. (2006b) but A. ervi has never been recorded as a member of this complex. Similarly, although E. niger is a specific parasitoid of Uroleucon and Macrosiphoniella aphids (Rakhshani et al. 2011), literature data that associate it to Macrosiphum rosae (Mescheloff \& Rosen 1988) has never been confirmed.

The present study summarizes the presence of some important invasive aphid species and their aphidiine parasitoids as potential biocontrol agents in the target area. For example, Aphis illinoisensis has been determined as an invasive species in several Mediterranean countries including North Africa (Havelka et al. 2011; El-Gantiry et al. 2012), while Cinara cedri is an invasive species in Israel (Michelena et al. 2005), Saudi Arabia (Ahmad \& Bakr 2013), Turkey (Central part - Aslan et al. 2004; Uysal et al. 2004) and Yemen (Starý et al. 2013). With regards to host range pattern of aphid parasitoids, occurrence of both rare and common species in the target region depended on the distribution of their host plants and associated aphids. However, major parts of Algeria and Libya, as the largest countries in North Africa, and of Saudi Arabia in Western Asia, are not adequately explored for aphid parasitoids.

Regarding the large number of Aphidiinae species identified in various areas of Middle Eastern and North Africa, further taxonomic revision should be conducted on some species of complicated genera. For example, the most recent revision of the genus Lysiphlebus Förster, 1862 in Europe (Tomanović et al. 2018) revealed the existence of some new species, of which Lysiphlebus volkli Tomanović \& Kavallieratos is also widely present in Iran. Despite the recent revision by Tomanović et al. (2018), the taxonomic problem of the genus Lysiphlebus in the Palaearctic region has not been completely resolved. Among unresolved species, we propose Lysiphlebus marismortui Mescheloff \& Rosen as a junior synonym of Lysiphlebus confusus Tremblay \& Eady. The former has all diagnostic and general characters that fall into normal variability within populations of $L$. confusus. It is worthy noting that the records of Adialytus ambiguus from some countries (i.e., Algeria, Iraq), actually refer to Lysiphlebus ambiguus. The latter has been subsequently renamed as Lysiphlebus confusus (Tremblay \& Eady 1978). Therefore, we excluded these records from the current study. Adialytus ambiguus is a species that is strictly associated with Sipha aphids (Rakhshani et al. 2012a; Stanković et al. 2015).

The lack of an adequate number of clear diagnostic characters is one of the main difficulties in the identification of Aphidiinae (Rakhshani et al. 2012b; Tomanović et al. 2018). This issue is common for some species of the genera Aphidius and Praon. Thus, the use of molecular markers, geometric morphometric analyses and ecological observations is necessary to identify species and elucidate the taxonomic ambiguities in some species-groups with similar host range patterns (Tomanović et al. 2014; Milošević et al. 2015; Rakhshani et al. 2015; Tomanović et al. 2018).

On the basis of the current review, and the background data, additional field surveys are necessary to be conducted in the unexplored regions, especially within the isolated areas from desert lowlands to humid and mountaneous highlands in different countries of the Middle East and North Africa so as to shed light on the very complex plant-aphid-aphidiine tritrophic associations and to reveal new species. As numerous species have been recorded in the region of the Middle East and North Africa, the proposed key will be a useful tool for the forthcoming studies that will help the accurate identification of aphid parasitoids.

\section{Acknowledgements}

The contribution by E. Rakhshani was supported by the project UOZ-GR-9517-2 that was granted by the University of Zabol. The contribution of P. Starý was partially supported by institutional support. Zubair Ahmad extends his gratitude to the Research Center Advanced Materials Science (RCAMS), King Khalid University for funding through research program (RCAMS)-009/19. 


\section{References}

Abdel-Rahman M.A.A. 2005. The relative abundance and species composition of hymenopterous parasitoids attacking cereal aphids (Homoptera: Aphididae) infesting wheat plants at Upper Egypt. Egyptian Journal of Agricultural Research 83 (2): 633-645.

Abdel-Rahman M.A.A., Nasser M.A.K. \& Ali A.M. 2000. Incidence of hymenopterous parasitoids attacking cereal aphids in wheat fields in Upper Egypt. Assiut Journal of Agricultural Sciences 31 (2): $317-328$.

Abdel-Samad S.S. \& Ahmed M.A. 2009. Population fluctuations of Aphis craccivora and liriomyza trifolii and their endoparasitoids on faba bean varieties. In: Kumari S., Bayaa B., Makkouk K., El-Ahmed A., El-Heneidy A., Jamal M., Jboory I., Abou-Gharbieh W., Abu Irmaileh B., Choueiri E., Kfoury L., Haidar M., Dawabah A., Shehab A. \& Abu-Jawdeh Y. (eds) $10^{\text {th }}$ Arab Congress of Plant Protection: 131. Arab Society for Plant Protection, Beirut.

Abou-Fakhr E.M. \& Kawar N.S. 1998. Complex of endoparasitoids of aphids (Hom.: Aphididae) on vegetables and other plants. Entomologicheskoe Obozrenie 77: 753-763. [In Russian]

Abou-Fakhr I. 1982. Aphid Endoparasitoid Complex on Certain Vegetables Ornamentals and Weeds in Lebanon. Ph.D. thesis, American University of Beirut.

Adly D. \& El-Gantiry A. 2014. Defense reactions of the grape aphid Aphis illinoisensis (Hemiptera: Aphididae) to parasitoid species Lysiphlebus testaceipes (Hymenoptera: Braconidae) and Aphelinus albipodus (Hymenoptera: Aphelinidae). Journal of Crop Protection 3: 683-690.

Aghajanzadeh S., Rasulian G., Rezwani A. \& Esmaili M. 1995. Identification of the aphids attacking citrus trees in West-Mazandaran and their population dynamics. In: Proceedings of the 12th Iranian Plant Protection Congress Vol. 1: 208. University of Tehran, Karadj.

Ahmad Z. \& Bakr R.F.A. 2013. Biosystematics of aphid parasitoids (Hymenoptera: Braconidae: Aphidiinae) from Asir region of Saudi Arabia. World Journal of Applied Sciences and Research 3 (1): $23-26$.

Ahmadi R. 2000. Natural enemies of Russian wheat aphid Diuraphis noxia, in Hamadan province. In: Proceedings of the $14^{\text {th }}$ Iranian Plant Protection Congress Vol. 1: 226. Isfahan University of Technology, Isfahan.

Ahmadi, A.A. \& Sarafrazi, A.M. 1993. Distribution and natural enemies of Russian Wheat aphid, Diuraphis noxia in Fars province. In: Proceedings of the 11th Iranian Plant Protection Congress: Vol 1: 1. University of Guilan, Rasht.

Akar S. \& Erdoğan Ö.Ç. 2017. Contributions to Aphidiinae (Hymenoptera: Braconidae) fauna of Turkey with new records. Trakya University Journal of Natural Sciences 18 (2): 89-96.

https://doi.org/10.23902/trkjnat.282623

Alaoğlu O. 1994. Erzurum'da frenkuzumu (Ribes aureum L.) zararhsi Cryptomyzus ribis (L.) (Aphididae: Homoptera)'in parazitoit ve predator faunas. In: Proceedings of the $3^{\text {rd }}$ Turkish National Congress of Biological Control: 49-58. Ege Universitesi, Izmir

Al-Azawi A.F. 1970. Some aphid parasitoids from central and south Iraq with notes on their occurrence. Bulletin of Iraq Natural History Museum 4: 27-31.

Alikhani M., Rezwani A., Starý P., Kavallieratos N.G. \& Rakhshani E. 2013. Aphid parasitoids (Hymenoptera: Braconidae: Aphidiinae) in cultivated and non-cultivated areas of Markazi Province Iran. Biologia 68 (5): 966-973. https://doi.org/10.2478/s11756-013-0234-y 
Al-Rawy M.A., Kaddou I.K. \& Starý P. 1969. Predation of Chrysopa carnea Steph. on mummified aphids and its possible significance in population regulation (Neuroptera Hymenoptera Homoptera). Bulletin of Biological Research Centre (Baghdad) 4: 30-34.

Aslan B. \& Karaca I. 2005. Fruit tree Aphids and their natural enemies in Isparta region Turkey. Journal of Pest Science 78: 227-229. https://doi.org/10.1007/s10340-005-0097-2

Aslan M.M., Uygun N. \& Starý P. 2004. A survey of aphid parasitoids in Kahramanmaras Turkey (Hymenoptera: Braconidae; Aphidiinae; and Hymenoptera: Aphelinidae). Phytoparasitica 32 (3): 255263. https://doi.org/10.1007/BF02979820

Avci Ü. \& Özbek H. 1991. Natural enemies of the cabbage aphid Brevicoryne brassicae (L.) (Homoptera: Aphididae) in Erzurum. Turkish Journal of Entomology 15 (1): 37-41. [In Turkish].

Avidov Z. \& Harpaz L. 1969. Plant Pests of Israel. Israel Universities Press, Jerusalem.

Avidov Z. \& Kotter E. 1966. The pests of safflower Carthamus tinctoria L. in Israel. Scripta Hierosolymitana 18: 9-26.

Ayadi M., Starý P., Ben Halima K.M. \& Belkadi M.S. 2017. Aphid parasitoid species and their hosts in Western South of Tunisia (Hymenoptera: Braconidae: Aphidiinae; Hemiptera: Aphididae). Egyptian Journal of Biological Pest Control 27 (2): 223-226.

Babaee M.R., Sahragard A. \& Rezwani A. 2000. Three species of parasitoids (Aphidiidae) on forest trees aphids in Mazandaran and a new method for determining percent parasitism. In: Proceedings of the $14^{\text {th }}$ Iranian Plant Protection Congress Vol. 1: 128. Isfahan University of Technology, Isfahan.

Baer C.F., Tripp D.W., Bjorksten T.A. \& Antolin M.F. 2004. Phylogeography of a parasitoid wasp (Diaeretiella rapae): no evidence of host-associated lineages. Molecular Ecology 13: 1859-1869.

https://doi.org/10.1111/j.1365-294X.2004.02196.x

Bagheri-Matin S., Shahrokhi S. \& Starý P. 2010. Report of Praon gallicum (Hymenoptera: Braconidae: Aphidiinae) from Iran. Applied Entomology and Phytopathology 78: 33-34.

Barahoei H., Madjdzadeh S.M., Mehrparvar M. \& Starý P. 2010. A study of Praon Haliday (Hymenoptera: Braconidae: Aphidiinae) in south-east Iran with two new records. Acta Entomologica Serbica 15: 107120.

Barahoei H., Madjdzadeh S.M., Mehrparvar M. 2011. Morphometric differentiation of five biotypes of Lysiphlebus fabarum (Marshall) (Hymenoptera: Braconidae: Aphidiinae) in Iran. Zootaxa 2745 (1): $43-52$.

Barahoei H., Madjdzadeh S.M. \& Mehrparvar M. 2012. Aphid parasitoids (Hymenoptera: Braconidae: Aphidiinae) and their tritrophic relationships in Kerman province Southeastern Iran. Iranian Journal of Animal Biosystematics 8 (1): 1-14.

Barahoei H., Rakhshani E., Madjdzadeh S.M., Alipour A., Taheri S., Nader E., Mitrovski-Bogdanović A., Petrović-Obradović O., Starý P., Kavallieratos N.G. \& Tomanović Ž. 2013. Aphid parasitoid species (Hymenoptera: Braconidae: Aphidiinae) of central submountains of Iran. North Western Journal of Zoology 9 (1): 70-93.

Barahoei H., Rakhshani E., Nader E., Starý P., Kavallieratos N.G., Tomanović Ž. \& Mehrparvar M. 2014. Checklist of Aphidiinae parasitoids (Hymenoptera: Braconidae) and their host aphid associations in Iran. Journal of Crop Protection 3 (2): 199-232.

Barjadze S., Karaca I., Yasar B. \& Gratiashvili N. 2010. New evidence of parasitoids of pest aphids on roses and grapevine in Turkey (Hem.: Aphididae; Hym.: Braconidae: Aphidiinae). Journal of Entomology and Acarology Research 42 (3): 143-145. https://doi.org/10.4081/jear.2010.143 
Ben Halima K.M. 2011. Effectiveness of Lysiphlebus testaceipes Cresson as biocontrol agent of Aphis gossypii Glover infesting pepper plants. European Journal of Environmental Sciences 1 (1): 28-32. https://doi.org/10.14712/23361964.2015.62

Ben Halima K.M. \& Ben Hamouda M.H. 1993. Les pucerons des cultures protegees et leurs ennemis en Tunisie. Tropicultura 11 (2): 50-53.

Ben Halima K.M. \& Ben Hamouda M.H. 2005. A propos des pucerons des arbres fruitiers de Tunisie. Notes fauniques de Gembloux 58: 11-16.

Ben Halima K.M., Rabasse J.M. \& Ben Hamouda M.H. 1994. Les pucerons des agrumes et leurs ennemis en Tunisie. Tropicultura 12 (4): 145-147. Available from http://www.tropicultura.org/content/v12n4.html [accessed 3 Jul. 2019].

Ben Halima K.M., Mdellel L., Karboul H. \& Zouari S. 2013. Natural enemies of Hyalopterus pruni species complex in Tunisia. Tunisian Journal of Plant Protection 8 (2): 119-126.

Benhamacha M., Ghezali D., Chemala A. \& Marniche F. 2017. First report of Pauesia silana Tremblay, 1969 (Hymenoptera: Aphidiidae), a natural enemy of the aphid Cinara pini L., in Algeria. EPPO Bulletin 47: 283-284. https://doi.org/10.1111/epp.12387

Bleton C.A. \& Fieuzet L. 1939. Notes sur quelques insectes auxiliaires observés dans la région de Fès. Bulletin de la Société des sciences naturelles du Maroc 19: 57-65. Available from https://bibdigital.rjb.csic.es/idurl/1/15070 [accessed 3 Jul. 2019].

Bodenheimer F.S. \& Swirski E. 1957. The Aphidoidea of the Middle East. Weisman Science Press, Jerusalem.

Boivin G., Hance T. \& Brodeur J. 2012. Aphid parasitoids in biological control. Canadian Journal of Plant Science 92: 1-12. https://doi.org/10.4141/cjps2011-045

Boukhris-Bouhachem S. 2011. Aphid enemies reported from Tunisian citrus orchards. Tunisian Journal of Plant Protection 6: 21-27.

Chaouche S.T. \& Laamari M. 2015. Aphid parasitoid species (Hymenoptera: Braconidae: Aphidiinae) and their associations in Biskra province Algeria. Journal of Entomology and Zoology Studies 3 (2): $179-184$.

Charles J.J. \& Paine T.D. 2016. Fitness effects of food resources on the polyphagous aphid parasitoid Aphidius colemani Viereck (Hymenoptera: Braconidae: Aphidiinae). PloS One 11 (1): e0147551.

https://doi.org/10.1371/journal.pone.0147551

Chen J.H., González D. \& Luhman J. 1991. A new species of Aphidius (Hymenoptera) attacking the pea aphid, Acyrthosiphon pisum. Entomophaga 35 (4): 509-514. https://doi.org/10.1007/BF02375085

Cross A.E. \& Poswal M.A. 1996. Dossier on Pauesia antennata (Mukerji): Biological Control Agent for the Brown Peach Aphid Pterochloroides persicae, in Yemen. International Institute of Biological Control Ascot Berkshire.

Darvish-Mojeni T. \& Bayat-Asadi H. 1995. Identification of natural enemies of wheat green aphid Sitobion avenae, in Gorgan and Dasht. In: Proceedings of the $12^{\text {th }}$ Iranian Plant Protection Congress Vol. 1: 21. University of Tehran, Karadj.

Darvish-Mojeni T. 1994. An introduction to one parasitoid wasp species and on new hyperparasitoid wasp genus for the fauna of Iran. Journal of Entomological Society of Iran 14: 79.

Das B.C. \& Chakrabarti S. 1990. New and little known aphidiid parasitoids (Hymenoptera: Aphidiidae) of gall forming aphids in western Himalaya, with notes on their seasonal history. Oriental Insects 24 (1): 399-414. https://doi.org/10.1080/00305316.1990.11835548 
Davatchi A. \& Shojai M. 1968. Entomophagous Hymenopterous Wasps of Iran. University of Tehran Faculty of Agriculture.

Davidian E.M. 2005. A review of species of the subgenus Trioxys s. str., genus Trioxys Haliday (Hymenoptera: Aphidiidae) of Russia and adjacent countries. Entomological Review 84 (3): 579-609.

Düzgüneş Z., Toros S., Kilincer N. \& Kovanci B. 1982. The Parasites and the Predators of Aphidoidea in Ankara. Turkish Ministry of Agriculture Ankara. [In Turkish].

Eady R.D. 1969. A new diagnostic character in Aphidius (Hymenoptera: Braconidae) of special significance in species on pea aphid. Systematic Entomology 38: 165-173.

https://doi.org/10.1111/j.1365-3113.1969.tb00224.x

El-Gantiry A.M., El-Heneidy A.H., Mousa S.F. \& Adly D. 2012. Aphis illinoisensis Shimer (Hemiptera: Aphididae) a recent invasive aphid species in Egypt. Egyptian Journal of Biological Pest Control 22 (2): 225-226.

El-Ghiet U.M.A., Edmardash Y.A.E. \& Gadallah N.S. 2014. Braconidae diversity (Hymenoptera: Ichneumonoidea) in alfalfa fields Medicago sativa L. of some Western Desert Oases. Egyptian Journal of Crop Protection 3 (4): 543-556.

El-Heneidy A.H. 1991. Seasonal abundance of aphids and their natural enemies in wheat fields in Upper Egypt. Egyptian Journal of Biological Pest Control 1 (1): 5-10.

El-Heneidy A.H. 1994. Efficacy of aphidophagous insects against aphids at wheat fields in Egypt, a 5year evaluation. Egyptian Journal of Biological Pest Control 4 (2): 113-123.

El-Heneidy A.H. \& Abdel-Samad S.S. 2001. Tritrophic interaction among Egyptian wheat plant, cereal aphids and natural enemies. Egyptian Journal of Biological Pest Control 11 (2): 119-125.

El-Heneidy A.H. \& Attia A.A. 1988/1989. Evaluation to the role of parasitoids and predators associated with aphids in wheat fields Egypt. Bulletin of Entomological Society of Egypt 17: 137-147.

El-Heneidy A.H., González D., Starý P., Adly D. \& El-Khawas M.A. 2001. A survey of primary and secondary parasitoid species of cereal aphids on wheat in Egypt. Egyptian Journal of Biological Pest Control 11 (2): 193-194.

El-Heneidy A.H., González D., Starý P. \& Adly D. 2002. Significance of hyperparasitization of primary cereal aphid parasitoids in Egypt "Hymenoptera Parasitic". Egyptian Journal of Biological Pest Control 12 (2): 109-114.

El-Heneidy A.H., Agamy E.A., El-Husseini M.M. \& Adly D. 2003. Seasonal occurrence of the aphid parasitoid Aphidius matricariae Hal. (Hymenoptera: Aphidiidae) in Egyptian wheat fields. Agricultural Research Journal Suez Canal University 2 (1): 103-108.

El-Heneidy A.H., Sobhy H.M., Abd-El-Wahed S.M.N. \& Mikhail W.Z.A. 2004. Biological aspects and life table analysis of cereal aphid species and their parasitoid Aphidius colemani Viereck (Hymenoptera: Aphidiidae). Egyptian Journal of Biological Pest Control 14 (1): 43-51.

Elmali M. 1997. Sipha (Rungsia) elegans in Konya Province of Turkey. Annales ANPP 3 (3): 903-910.

Elmali M. \& Toros S. 1994. Investigations on the natural enemies of aphids damaging to wheat in Konya province. In: Proceedings of the $3^{\text {rd }}$ Turkish National Congress of Biological Control: 13-29. Ege Universitesi, Izmir.

El-Serafy H.A. 1999. Population density of cereal aphids' parasitoids and their role in suppressing cereal aphids on wheat plantations at Mansoura district. Archiv für Phytopathologie und Pflanzenschutz 32 (3): 257-264. https://doi.org/10.1080/03235409909383295 
Erdoğan Ö.Ç., Tomanović Ž. \& Beyarslan A. 2008. New aphid parasitoids (Hymenoptera: Braconidae: Aphidiinae) in the region of Marmara Turkey. Acta Entomologica Serbica 13 (1-2): 85-88.

Erdoğan Ö.Ç. \& Akar S. 2018. First record of the species Adialytus veronicaecola (Starý, 1978) (Hymenoptera: Braconidae: Aphidiinae) from the West Palaearctic Region. Plant Protection Bulletin 58 (4): 227-230. [In Turkish]. https://doi.org/10.16955/bitkorb.396011

Erkin E. 1983. Investigation on the hosts, distribution and efficiency of the natural enemies of the family Aphididae (Homoptera) harmful to pome and stone fruit trees in Izmir province of Aegean region. Türkiye Bitki Koruma Dergisi 7: 29-49.

Fabre J.P. \& Rabasse J.M. 1987. Introduction dans le Sud-Est de la France d'un parasite: Pauesia cedrobii [Hym.: Aphidiidae] du puceron: Cedrobium laportei [Hom.: Lachnidae] du cèdre de l'atlas: Cedrus atlantica. Entomophaga 32: 127-141. https://doi.org/10.1007/BF02373123

Farahani S., Talebi A.A. \& Barahoei H. 2015. Occurrence of the rare aphid parasitoid Praon bicolor Mackauer, 1959 (Hymenoptera: Braconidae: Aphidiinae) in central Asia. Journal of Insect Biodiversity and Systematics 1 (1): 11-15.

Farahani S., Talebi A.A. \& Rakhshani E. 2016. Iranian Braconidae (Insecta: Hymenoptera: Ichneumonoidea): diversity, distribution and host association. Journal of Insect Biodiversity and Systematics 2 (1): 1-92.

Farahani S., Talebi A.A., Starý P. \& Rakhshani E. 2017. Occurrence of the rare root aphid parasitoid Aclitus obscuripennis (Hymeoptera: Braconidae: Aphidiinae) in Iran. Biologia 72 (12): 1494-1498. https://doi.org/10.1515/biolog-2017-0167

Farahbakhsh G. 1961. A checklist of economically important insects and other enemies of plants and agricultural products of Iran. Ministry of Agriculture Department of Plant Protection Tehran.

Favret C. 2019. Aphid Species File. Version 5.0. Available from http://aphid.speciesfile.org [accessed 3 February 2019].

Fleschner C.A. 1963. Releases of recently imported insect parasites and predators in California, 196061. Pan-Pacific Entomologist 39: 114-116.

Gadallah N.S., El-Heneidy A.H., Mahmoud S.M. \& Kavallieratos N.G. 2017. Identification key, diversity and host associations of parasitoids (Hymenoptera: Braconidae: Aphidiinae) of aphids attacking cereal crops in Egypt. Zootaxa 4312 (1): 143-154. https://doi.org/10.11646/zootaxa.4312.1.6

Gärdenfors U. 1986. Taxonomic and biological revision of Palaearctic Ephedrus Haliday (Hymenoptera: Braconidae: Aphidiinae). Entomologia Scandinavica (Supplement) 27: 1-95.

Ghafouri Moghaddam M., Rakhshani E., Starý P., Tomanović Ž. \& Kavallieratos N.G. 2012. Occurrence of Monoctonia vesicarii Tremblay (Hym.: Braconidae: Aphidiinae), a very rare parasitoid of the gall forming aphids Pemphigus spp. (Hemi., Eriosomatidae) in Iran. In: Sarafrazi A., Asef M.R., Mozhdehi M., Mozhdehi M., Solhjouy Fard S. \& Abdollahi T. (eds) Proceedings of the $20^{\text {th }}$ Iranian Plant Protection Congress Vol. 1: 212. University Shiraz, Shiraz.

Ghaliow M.E., Petrović A., Kocić K., Čkrkić J., Bogdanović A.M., Starý P., Kavallieratos N.G. \& Tomanović Ž. 2018. Key for identification of the parasitoids (Hymenoptera: Braconidae: Aphidiinae) of aphids infesting alfalfa in Europe. Zootaxa 4378 (1): 98-110. https://doi.org/10.11646/zootaxa.4378.1.6

Ghanim A. \& El-Adl M. 1983. Aphids infesting wheat and the effect of their predators in suppressing their populations in fields at Mansoura district Egypt. Journal of Agriculctural Science 8 (4): 958-968. 
González D., White W., Hall J. \& Dickson R.C. 1978. Geographical distribution of Aphidiidae (Hym.) imported to California for biological control of Acyrthosiphon kondoi and Acyrthosiphon pisum (Hom.: Aphididae). Entomophaga 23: 239-248. https://doi.org/10.1007/BF02373098

González D., Gilstrop F., McKinnon L., Zhang J., Zareh N., Zhang G., Starý P., Wooley J. \& Wang R. 1992. Foreign exploration for natural enemies of Russian wheat aphid in Iran and in the Kunlun, Tian Shan, and Altai Mountain Valleys of The People's Republic of China. In: Proceedings of Russian Wheat Aphid Conference: 197-208. Great Plains Agriculture Council Publications, Texas.

Gülçlü C. \& Özbek H. 2002. Effects of the parasitoids Aphidius ervi Hal. and Praon dorsale Hal. (Hymenoptera: Aphidiidae) on Metopolophium dirhodum (Walker) (Homoptera Aphididae), pest of rosehip (Rosa spp.) in Erzurum. In: Özbek H., Güçlü Ş. and Hayat R. (eds) Proceedings of Fifth Turkish National Congress of Biological Control: 81-88. Ataturk University, Erzurum.

Güz N. \& Kilinçer N. 2005. Aphid parasitoids (Hymenoptera: Braconidae: Aphidiinae) on weeds from Ankara Turkey. Phytoparasitica 33 (4): 359-366. https://doi.org/10.1007/BF02981302

Hafez A.A. 1994. Increasing the role of biocontrol agents against cereal aphids infesting wheat in Qalubia-Egypt. Egyptian Journal of Biological Pest Control 4 (2): 57-71.

Hafez M. 1965. Characteristics of the open empty mummies of the cabbage aphid Brevicoryne brassicae (L.) indicating the identity of the emerged parasites. Agricultural Research Review 43 (4): 85-88.

Hågvar E.B. \& Hofsvang T. 1991. Aphid parasitoids (Hymenoptera: Aphidiidae): biology, host selection, and use in biological control. Biocontrol News and Information 12: 13-41.

Harpaz L. 1955. Bionomics of Therioaphis maculata (Buckton) in Israel. Journal of Economic Entomology 48: 668-671. https://doi.org/10.1093/jee/48.6.668

Hasan H.S. 2016. Survey of aphid species and associated parasitoids in Al-Homra Jordan. Journal of Entomology and Zoology Studies 4 (5): 1-4.

Hassan M.S. 1957. Studies on the damage and control of Aphis maidis Fitch, in Egypt (Hemiptera: Homoptera: Aphididae). Bulletin of the Entomological Society of Egypt 41: 213-230.

Hassan M.S. 1963. Natural Enemies of Some Root Aphids, in Egypt (Hemiptera-Homoptera: Aphididae). Cairo, General Organisation for Goverment Printing Offices.

Havelka J., Shukshuk A.H., Ghaliow M.E., Laamari M., Kavallieratos N.G., Tomanović Ž., Rakhshani E., Pons X. \& Starý P. 2011. Review of invasive grapevine aphid Aphis illinoisensis Shimer, and native parasitoids in the Mediterranean (Hemiptera: Aphididae; Hymenoptera: Braconidae: Aphidiinae). Archives of Biological Sciences Belgrade 63 (1): 269-274. https://doi.org/10.2298/ABS1101269H

Hawkins B.A. 1994. Pattern and Process in Host Parasitoid Interactions. Cambridge University Press, Cambridge.

Heidari Latibari M., Moravej G., Ghafouri Moghaddam M., Barahoei H. \& Hanley G.A. 2019. The novel host associations for the aphid parasitoid, Pauesia hazratbalensis (Hymenoptera, Braconidae, Aphidiinae). Oriental Insects 10: 1-8. https://doi.org/10.1080/00305316.2019.1586780

Hodjat S.H. \& Moradeshaghi M.J. 1988. Citrus aphids of Iran. Journal of Plant Protection 31: 1-40.

Houerou H.N.L. 2000. Restoration and rehabilitation of arid and semiarid Mediterranean ecosystems in North Africa and West Asia: a review. Arid Soil Research and Rehabilitation 14 (11): 3-14.

https://doi.org/10.1080/089030600263139

Hussein M.K. \& Kawar N.S. 1984. A study of aphids and their natural enemies in Southern Lebanon. Arab Journal of Plant Protection 2: 17-82. [In Arabic]. 
Ibrahim A.M.A. \& Afifi A.I. 1991. The relationship between cereal aphids and aphidophagous syrphids, coccinellids and chrysopids on wheat and barley in Egypt. Bulletin of the Faculty of Agriculture Cairo University 42 (1): 151-166.

Ibrahim A.M.A. 1990a. Corn leaf aphid Rhopalosiphum maidis (F) (Hom.: Aphididae) on wheat and associated primary parasitoids and hyperparasitoids. Bulletin of the Entolomological Society of Egypt 69: 149-157.

Ibrahim A.M.A. 1990b. Population dynamics of bird-cherry aphid Rhopalosiphum padi L. (Hom.: Aphididae) and its primary parasitoids and hyperparasitoids association on wheat in Egypt. Bulletin of the Entolomological Society of Egypt 69: 137-147.

Irshaid L.A. \& Hasan H.S. 2011. Bioresidual effect of two insecticides on melon aphid Aphis gossypii Glover (Homoptera: Aphididae) and its parasitoid Aphidius colemani Viereck (Hymenoptera: Brachonidae). American-Eurasian Journal of Agricultural \& Environmental Sciences 11 (2): 228-236.

Jafari N. \& Modarres Awal M. 2012. Aphid parasitoids associations on stone fruit trees in Khorasan-eRazavi province (Iran) (Hymenoptera: Braconidae: Aphidiinae). Munis Entomology and Zoology 7 (1): $418-423$.

Jafari N., Karimi J., Modarres Awal M. \& Rakhshani E. 2011. Morphological and molecular methods in identification of Aphidius transcaspicus Telenga (Hym: Braconidae: Aphidiinae) parasitoid of Hyalopterus spp. (Hom.: Aphididae) with additional data on Aphidiinae phylogeny. Journal of the Entomological Research Society 13 (2): 91-103.

Karaat S. \& Goven M.A. 1986. General status of natural enemies of green peach aphid (Myzus persicae Sulz.) in tobacco areas in southeastern Anatolia. In: Proceedings of the $1^{\text {st }}$ Turkish National Congress of Biological Control: 162-172. Çukurova Üniversitesi, Adana.

Kargarian F., Hesami S. \& Rakhshani E. 2016. First report of Monoctonia pistaciaecola (Hymenoptera: Braconidae) from Iran. Journal of Entomological Research 8 (3): 263-267. [In Persian.]

Kavallieratos N.G., Lykouressis D.P., Sarlis G.P., Stathas G.J., Sanchis Segovia A. \& Athanassiou C.G. 2001. The Aphidiinae (Hymenoptera: Ichneumonoidea: Braconidae) of Greece. Phytoparasitica 29 (4): 306-340. https://doi.org/10.1007/BF02981847

Kavallieratos N.G., Tomanović Z., Starý P., Athanassiou C.G., Sarlis G.P., Petrovic O., Niketic M. \& Veroniki M.A. 2004. A survey of aphid parasitoids (Hymenoptera: Braconidae: Aphidiinae) of Southeastern Europe and their aphid-plant associations. Applied Entomology and Zoology 39 (3): 527563. https://doi.org/10.1303/aez.2004.527

Kavallieratos N.G., Tomanović Ž., Athanassiou C.G., Starý P., Žikić V., Sarlis G.P. \& Fasseas C. 2005. Aphid parasitoids (Hymenoptera: Braconidae: Aphidiinae) infesting cotton, citrus, tobacco and cereal crops in southerneastern Europe: aphid-plant associations and keys. The Canadian Entomologist 137 (5): 516-531. https://doi.org/10.4039/n05-020

Kavallieratos N.G., Tomanović Ž., Starý P., Žikić V. \& Petrović Obradović V. 2010. Parasitoids (Hymenoptera: Braconidae: Aphidiinae) attacking aphids feeding on Solanaceae and Cucurbitaceae crops in southeastern Europe: aphidiine-aphid-plant associations and key. Annals of the Entomological Society of America 103 (2): 153-164. https://doi.org/10.1603/AN09004

Kavallieratos N.G., Tomanović Ž., Petrović A., Janković M., Starý P., Yovkova M. \& Athanassiou C.G. 2013. Review and key for the identification of parasitoids (Hymenoptera: Braconidae: Aphidiinae) of aphids infesting herbaceous and shrubby ornamental plants in Southeastern Europe. Annals of the Entomological Society of America 106 (3): 294-309. https://doi.org/10.1603/AN12090 
Kavallieratos N.G., Tomanović Ž., Petrović A., Kocić K., Janković M. \& Starý P. 2016. Parasitoids (Hymenoptera: Braconidae: Aphidiinae) of aphids feeding on ornamental trees in southeastern Europe: key for identification and tritrophic associations. Annals of the Entomological Society of America 109 (3): 473-487. https://doi.org/10.1093/aesa/saw003

Kazemzadeh S., Rakhshani E., Tomanović Ž., Starý P. \& Petrović A. 2009. Areopraon lepelleyi (Waterston) (Hymenoptera: Braconidae: Aphidiinae), a parasitoid of Eriosomatinae (Hemiptera: Aphidoidea: Pemphigidae) new to Iran. Acta Entomologica Serbica 14: 55-63.

Kilinçer N. 1982. Der Parasitenkomplex der Mehligen Kohlblattlaus in Ankara umfasst zwei Parasitenund vier Hyperparasitenarten. Plant Protection Bulletin 22, 1-12. [In Turkish.]

Kittel R.N. 2016. Eighty-nine replacement names for Braconidae and Ichneumonidae (Insecta: Hymenoptera: Ichneumonoidea). Japanese Journal of Systematic Entomology 22 (2): 161-174.

Laamari M. \& Coeur d'Acier A. 2010. The grapevine aphid Aphis illinoisensis now found in Algeria. EPPO Bulletin 40: 167-168. [In French.] https://doi.org/10.1111/j.1365-2338.2009.02368.x

Laamari M., Tahar C.S., Benferhat S., Abbès S.B., Merouani H., Ghodbane S., Khenissa N. \& Starý P. 2011. Interactions tritrophiques: plante-puceron-hyménoptère parasitoïde observées en milieux naturels et cultivés de l'Est Algérien. Entomologie Faunistique 63 (3): 115-120.

Laamari M., Chaouche S.T., Halimi C.W., Benferhat S., Abbes S.B., Khenissa N. \& Starý P. 2012. A review of aphid parasitoids and their associations in Algeria (Hymenoptera: Braconidae: Aphidiinae; Hemiptera: Aphidoidea). African Entomology 20 (1): 161-170. https://doi.org/10.4001/003.020.0120

Laamari M., Boughida S. \& Merouani H. 2016. Distribution, parasitoids and cyclic appearance of Russian wheat aphid Diuraphis noxia (Mordvilko, 1913) (Hemiptera Aphididae) in Algeria. European Journal of Environmental Sciences 6 (2): 103-107. https://doi.org/10.14712/23361964.2016.15

Labdaoui Z.E. \& Guenaoui Y. 2018. Some observations on the predominance of Aphis spiraecola on citrus in Northwestern Algeria. Tunisian Journal of Plant Protection 13: 147-157.

Lokeshwari D., Kumar N.K. \& Rakhshani E. 2016. Parasitoids (Hymenoptera: Braconidae: Aphidiinae) of Aphis gossypii (Hem., Aphididae) in Bengaluru India. Journal of Insect Biodiversity and Systematics 1 (2): 155-163.

Lozier D.J., Roderick K.G. \& Mills J.N. 2008. Evolutionarily significant units in natural enemies: Identifying regional populations of Aphidius transcaspicus (Hymenoptera: Braconidae) for use in biological control of mealy plum aphid. Biological Control 46: 532-541.

https://doi.org/10.1016/j.biocontrol.2008.05.002

Ma K., Chen G., Liu B., Chen B., Amin R., Zhang J. \& Chun M. 2010. The First Asian Plant Conservation Report: A Review of Progress in Implementing the Global Strategy for Plant Conservation. Chinese Academy of Sciences, Beijing.

Mackauer M. 1960. Trioxys (Trioxys) vandenboschi n.sp., ein neuer Blattlaus - Parasit aus dem Iran (Hymenoptera: Braconidae: Aphidiinae). Senckenbergiana Biologica 41 (5-6): 359-362.

Mackauer M. 1961. Neue europaeische Blattlatus-Schlupfwespen (Hymenoptera: Aphidiidae). Bollettino del Laboratorio di Entomologia Agraria 'Filippo Silvestri' 19: 270-290.

Mackauer M. 1963. A re-examination of C. F. Baker's collection of aphid parasites (Hymenoptera: Aphidiidae). Canadian Entomologist 95: 921-935. https://doi.org/10.4039/Ent95921-9

Mackauer M. \& Finlayson T. 1967. The hymenopterous parasites (Hymenoptera: Aphidiidae et Aphelinidae) of the pea aphid in eastern North America. Canadian Entomologist 99 (10): 1051-1082. https://doi.org/10.4039/Ent991051-10 
Mackauer M. \& Starý P. 1967. Hymenoptera Ichneumonoidea: World Aphidiidae. In: Delucchi V \& Remaudière G. (eds) Index of Entomophagous Insects: 1-195. Le Francois, Paris.

Marsh P.M. 1977. Notes on the taxonomy and nomenclature of Aphidius species [Hym.: Aphidiidae] parasitic on the pea aphid in North America. Entomophaga 2 2(4): 365-372.

https://doi.org/10.1007/BF02373261

Mdellel L. \& Ben Halima K.M. 2015. Apple aphid's species and their natural enemies in Tunisian orchards. Journal of New Science Agriculture and Biotechnology 24 (4): 1108-1114.

Mdellel L., Ben Halima M. \& Rakhshani E. 2015. Laboratory evaluation of Pauesia antennata (Hymenoptera: Braconidae), specific parasitoid of Pterochloroides persicae (Hemiptera: Aphididae). Journal of Crop Protection 4 (3): 385-393.

Mehrparvar M., Hatami B. \& Starý P. 2005. Report of Aphidius rosae (Hym.: Braconidae), a parasitoid of rose aphid Macrosiphum rosae (Hom.: Aphididae) from Iran. Journal of Entomological Society of Iran 25 (1): 63-64.

Mescheloff E. \& Rosen D. 1988. Biosystematic studies on the Aphidiidae of Israel (Hymenoptera: Ichneumonoidea), 2. The genera Ephedrus and Praon. Israel Journal of Entomology 22: 75-100.

Mescheloff E. \& Rosen D. 1990a. Biosystematic studies on the Aphidiidae of Israel (Hymenoptera: Ichneumonoidea), 3. The genera Adialytus and Lysiphlebus. Israel Journal of Entomology 24: 35-50.

Mescheloff E. \& Rosen D. 1990b. Biosystematic studies on the Aphidiidae of Israel (Hymenoptera: Ichneumonoidea), 4. The genera Pauesia Diaeretus Aphidius and Diaeretiella. Israel Journal of Entomology 24: 51-91.

Mescheloff E. \& Rosen D. 1993. Biosystematic studies on the Aphidiidae of Israel (Hymenoptera: Ichneumonoidea), 5. The genera Trioxys and Binodoxys. Israel Journal of Entomology 27: 31-47.

Michelena M.J., Assael F. \& Mendel Z. 2005. Description of Pauesia (Pauesia) anatolica (Hymenoptera: Braconidae: Aphidiinae) sp. nov., a parasitoid of the Cedar aphid Cinara cedri. Phytoparasitica 33 (5): 499-505. https://doi.org/10.1007/bf02981399

Milošević M.I., Petrović A., Stanković S.S., Čkrkić J., Starý P., Žikić V. \& Tomanović Ž. 2015. Taxonomic position and phylogenetic relationships of the genera and species Euaphidius and Remaudierea (Hymenoptera: Braconidae: Aphidiinae) analyzed using molecular markers and geometric morphometrics. Annals of the Entomological Society of America 108 (3): 435-445.

https://doi.org/10.1093/aesa/sav018

Mimeur J. 1934. Aphididae of Morocco. Mémoires de la Société des sciences naturelles du Maroc 40: $1-17$.

Mitrović M., Petrović A., Kavallieratos N.G., Starý P., Petrović-Obradović O., Tomanović Ž. \& Vorburger C. 2013. Geographic structure with no evidence for host-associated lineages in European populations of Lysiphlebus testaceipes, an introduced biological control agent. Biological Control 66 (3): 150-158. https://doi.org/10.1016/j.biocontrol.2013.05.007

Mohammadbeigi A. 2000. Natural enemies of the walnut aphids in Qazvin province. Proceedings of the $14^{\text {th }}$ Iranian Plant Protection Congress Vol. 1: 278. Isfahan University of Technology, Isfahan.

Mokhtari A., Sahragard A., Rezwani A. \& Salehi L. 2000. Study on five parasitoid species of the stone fruit aphids in Gilan province. Proceedings of the 14 ${ }^{\text {th }}$ Iranian Plant Protection Congress, Vol. 1: 273. Isfahan University of Technology, Isfahan.

Monajemi N. \& Esmaili M. 1981. Population dynamics of alfalfa aphids and their natural controling factors, in Karadj. Journal of Entomological Society of Iran 6 (1-2): 41-63. 
Mosavi H., Havaskary M., Monem R., Khodaparast S. \& Sakenin H. 2012. On a collection of Braconidae (Hymenoptera) from East Azarbaijan and vicinity Iran. Calodema 226: 1-4.

Mossadegh M.S., Starý P. \& Salehipour H. 2011. Aphid parasitoids in a dry lowland area of Khuzestan Iran (Hymenoptera: Braconidae: Aphidiinae). Asian Journal of Biological Science 4: 175-181. https://doi.org/10.3923/ajbs.2011.175.181

Nazari Y., Zamani A.A., Masoumi S.E., Rakhshani E., Petrović-Obradović O., Tomanović S., Starý P. \& Tomanović Ž. 2012. Diversity and host associations of aphid parasitoids (Hymenoptera: Braconidae: Aphidiinae) in the farmlands of western Iran. Acta Entomologica Musei Nationalis Pragae 55 (2): 559584.

Nieto Nafria J., Favret C., Akimoto S., Barbagallo S., Chakrabarti S., Mier Durante M., Miller G.A., Qiao G.X., Sano M., Pérez Hidalgo N., Stekolshchikov A. \& Wegierek P. 2011. Register of genus-group taxa of Aphidoidea. In: Nieto Nafria J.M. \& Favret C. (eds) Registers of Family-Group and GenusGroup Taxa of Aphidoidea (Hemiptera: Sternorrhyncha): 481-635. Universidad de Leon, Leon.

Ölmez S. \& Ulusoy M.R. 2003. A survey of aphid parasitoids (Hymenoptera: Braconidae: Aphidiinae) in Diyarbakir Turkey. Phytoparasitica 31 (5): 524-528. https://doi.org/10.1007/BF02979745

Özder N. \& Toros S. 1999. Investigations on the natural enemies of aphid species damaging to wheat plants in Tekirdag Province. In: Proceedings of the $4^{\text {th }}$ Turkish National Congress of Biological Control: 501-512. Çukurova Üniversitesi, Adana.

Pike K.S., Allison D., Tanigoshi L.K., Harwood R.F., Clement S.L., Halbert S.E., Smith C.M., Johnson J.B., Reed G.L. \& Zwer P.K. 1991. Russian Wheat Aphid Biology Damage and Management. Washington Oregon Idaho Pacific Northwest Ext. Publ. PNW371, Pullman Washington.

Powell W., Budenberg W.J., Decker U.M. \& Wright A.F. 1990. Conservation and manipulation of aphid parasitoids. In: Proceedings of the Brighton Crop Protection Conference Pests and Diseases Vol. 2: 685-690. British Crop Protection Council, Brighton.

Radjabi G. 1989. Insects Attacking Rosaceous Fruit Trees in Iran. Vol. 3: Homoptera. Plant Pests and Diseases Research Institute, Tehran.

Ragab M.E. 1996. Biology and efficiency of Trioxys angelicae Hal. (Hymenoptera: Aphidiidae) a newly recorded parasitoid of Aphis craccivora Koch (Homoptera: Aphididae) in Egypt. Egyptian Journal of Biological Pest Control 6 (1): 7-11.

Ragab M.E., Gahanim A.A., Abou EL-Naga A.M. \& Saleh A.A. 2002. Effect of host aphid species, temperature and food supply on some biological characteristics of the two aphid parasitoids Diaeretiella rapae and Aphidius sp. (Hymenoptera: Aphidiidae). Journal of Agricultural Science (Mansoura University) 27 (7): 4997-5002.

Rakhshani E. 2012. Aphid parasitoids (Hym.: Braconidae: Aphidiinae) associated with pome and stone fruit trees in Iran. Journal of Crop Protection 1 (2): 81-95.

Rakhshani E., Talebi A.A., Kavallieratos N. \& Fathipour Y. 2004. Host stage preference, juvenile mortality and functional response of Trioxys pallidus (Hymenoptera: Aphidiinae). Biologia 59: 197-203.

Rakhshani E., Talebi A.A., Kavallieratos N.G., Rezwani A., Manzari S. \& Tomanović Ž. 2005a. Parasitoid complex (Hymenoptera: Braconidae: Aphidiinae) of Aphis craccivora Koch (Hemiptera: Aphidoidea) in Iran. Journal of Pest Science 78: 193-198. https://doi.org/10.1007/s10340-004-0080-3

Rakhshani E., Talebi A.A., Starý P., Manzari S. \& Rezwani A. 2005b. Re-description and biocontrol information of Pauesia antennata (Mukerji) (Hym.: Braconidae: Aphidiinae), parasitoid of Pterochloroides persicae (Chol.) (Hom.: Aphidoidea: Lachnidae). Journal of Entomological Research Society 7 (3): 61-71. 
Rakhshani E., Talebi A.A., Manzari S., Rezwani A. \& Rakhshani H. 2006a. An investigation on alfalfa aphids and their parasitoids in different parts of Iran, with a key to the parasitoids (Hemiptera: Aphididae; Hymenoptera: Braconidae: Aphidiinae). Journal of Entomological Society of Iran 25 (2): 1-14.

Rakhshani E., Talebi A.A., Starý P., Tomanović Ž., Manzari S. \& Kavallieratos N. 2006b. A new species of Aphidius Nees, 1818 (Hymenoptera: Braconidae: Aphidiinae) attacking Uroleucon aphids (Homoptera: Aphididae) from Iran and Iraq. Journal of Natural History 40 (32-34): 1923-1929. https://doi.org/10.1080/00222930601046451

Rakhshani E., Talebi A.A., Manzari S.,Tomanović Ž., Starý P. \& Rezwani A. 2007a. Preliminary taxonomic study of genus Praon Haliday (Hymenoptera: Braconidae: Aphidiinae) and its host associations in Iran. Journal of Entomological Society of Iran 26 (2): 19-34.

Rakhshani E., Talebi A.A., Starý P., Tomanović Ž. \& Manzari S. 2007b. Aphid-parasitoid (Hymenoptera: Braconidae: Aphidiinae) associations on willows and poplars in the biocorridors of Iran. Acta Zoologica Academiae Scientiarum Hungaricae 53: 281-297.

Rakhshani E., Talebi A.A., Starý P., Tomanović Ž., Kavallieratos N.G. \& Manzari S. 2008a. A review of Aphidius Nees in Iran: host associations, distribution, and taxonomic notes. Zootaxa 1767: 37-54.

Rakhshani E., Tomanović Ž. Starý P., Talebi A.A., Kavallieratos N.G., Zamani A.A. \& Stanković. S. 2008b. Distribution and diversity of wheat aphid parasitoids (Hymenoptera: Braconidae: Aphidiinae) in Iran. European Journal of Entomology 105: 863-870. https://doi.org/10.14411/eje.2008.114

Rakhshani E., Kazemzadeh S., Talebi A.A., Starý P., Manzari S., Rezwani A. \& Asadi G. 2008c. Preliminary taxonomic study of genus Trioxys Haliday (Hym.: Braconidae: Aphidiinae) and its host associations in Iran. In: Manzari Sh. (ed) Proceedings of the $18^{\text {th }}$ Iranian Plant Protection Congress Vol. 1: 97. Bu-Ali Sina University, Hamadan.

Rakhshani E., Talebi A.A., Manzari S., Starý P., Tomanović Ž. \& Arjmandi A.A. 2008d. Lipolexis gracilis Förster (Hymenoptera: Braconidae: Aphidiinae), an aphid parasitoid newly determined in Iran and Turkey. In: Manzari Sh. (ed) Proceedings of the $18^{\text {th }}$ Iranian Plant Protection Congress Vol. 1: 98. Bu-Ali Sina University, Hamadan.

Rakhshani E., Tomanović Ž., Starý P., Kavallieratos N.G., Ilić S.M., Stanković S. \& Rajabi-Mazhar N. 2011. Aphidiinae parasitoids (Hymenoptera: Braconidae) of Macrosiphoniella aphids (Hemiptra: Aphididae) in the western Palaearctic region. Journal of Natural History 45: 2560-2576.

https://doi.org/10.1080/00222933.2011.597004

Rakhshani E., Starý P. \& Tomanović Ž. 2012a. Species of Adialytus Förster, 1862 (Hymenoptera: Braconidae: Aphidiinae) in Iran: taxonomic notes and tritrophic associations. ZooKeys 221: 81-95. https://doi.org/10.3897/zookeys.221.3541

Rakhshani E., Kazemzadeh S., Starý P., Barahoei H., Kavallieratos N.G., Ćetković A., Popović A., Bodlah I. \& Tomanović Ž. 2012b. Parasitoids (Hymenoptera: Braconidae: Aphidiinae) of northeastern Iran: Aphidiine-aphid-plant associations, key and description of a new species. Journal of Insect Science 12:143. https://doi.org/10.1673/031.012.14301

Rakhshani E., Starý P. \& Tomanović Ž. 2013. Tritrophic associations and taxonomic notes on Lysiphlebus fabarum (Marshall) (Hymenoptera: Braconidae: Aphidiinae), A keystone aphid parasitoid in Iran. Archives of Biological Science Belgrade 65 (2): 667-680. https://doi.org/10.2298/ABS1302667R

Rakhshani E., Starý P., Pérez Hidalgo N., Čkrkić J., Ghafouri Moghaddam M., Tomanović S., Petrović A. \& Tomanović Ž. 2015. Revision of the world Monoctonia Starý, parasitoids of gall aphids: taxonomy, distribution, host range, and phylogeny (Hymenoptera: Braconidae: Aphidiinae). Zootaxa 3905 (4): 474-488. https://doi.org/10.11646/zootaxa.3905.4.2 
Rakhshani E., Starý P. \& Davidian E. 2017. A taxonomic review of the subgenus Pauesiella Sedlag \& Starý, 1980 (Hym.: Braconidae: Aphidiinae). Plant Pest Research (Guilan University) 7 (3): 53-66.

Rasulian G. 1989. Evaluation of some natural enemies of alfaalfa aphids. In: Proceedings of the $9^{\text {th }}$ Iranian Plant Protection Congress Vol. 1: 16. Ferdowsi University, Mashhad.

Remaudière G. \& Remaudière M. 1997. Catalogue of the World's Aphididae. INRA, Paris.

Rohlf F.J. 2006. TPSDig. Ver. 2.05. Stony Brook NY, Department of Ecology and Evolution State University of New York.

Rosen D. 1967. The hymenopterous parasites and hyperparasites of aphids on citrus in Israel. Annals of the Entomological Society of America 60: 394-399. https://doi.org/10.1093/aesa/60.2.394

Rosen D. 1969. The parasites of coccids, aphids and aleyrodids on citrus in Israel: some zoogeographical considerations. Israel Journal of Entomology 4: 45-53.

Samara R.Y. \& Qubbaj T.A. 2012. Preliminary study of some aphid natural enemies of TulkarmNorthern West-Bank and their aphid-plant associations. International Journal of Agronomy and Plant Production 3 (4): 123-127.

Satar S., Satar G., Karacaoğlu M., Uygun N., Kavallieratos N.G., Starý P. \& Athanassiou C.G. 2014. Parasitoids and hyperparasitoids (Hymenoptera) on aphids (Hemiptera) infesting citrus in east Mediterranean region of Turkey. Journal of Insect Science 14 (178): 1-7.

https://doi.org/10.1093/jisesa/ieu040

Schimitschek E. 1944. Forstinsekten der Türkei und ihre Umwelt: Grundlagen der türkischen Forstentomologie. Volk \& Reich Verlag, Prag/Amsterdam/Berlin/Vienna.

Schlinger E.I. \& Mackauer M. 1963. Identity, distribution and hosts of Aphidius matricariae Haliday, an important parasite of the green peach aphid Myzus persicae (Hymenoptera: Aphidiidae; Homoptera: Aphidoidea). Annals of the Entomological Society of America 65: 648-653.

https://doi.org/10.1093/aesa/56.5.648

Sertkaya E. \& Yigit A. 2002. Aphid species injurious on wheat and their natural enemies in Antakya Province. In: Özbek H., Güçlü Ş. and Hayat R. (eds) Proceedings of the $5^{\text {th }}$ Turkish National Congress of Biological Control: 265-274. Ataturk University, Erzurum.

Sharkey M.J. \& Wharton R.A. 1997. Morphology and terminology. In: Wharton R.A., Marsh P.M. \& Sharkey M.J. (eds) Manual of the New World Genera of the Family Braconidae (Hymenoptera): 19-37. Special Publication 1, International Society of Hymenopterists. Washington DC.

Slman F.A.A. 2006. Incidence of cereal aphids and seasonal abundance of their parasitoids in wheat fields in Sohag (Upper Egypt). Assiut Journal of Agricultural Science 37: 211-220.

Sobhy H.M., El-Heneidy A.H., Abd-El-Wahed S.M.N. \& Mikhail W.Z.A. 2004. Seasonal occurrence of aphid parasitoids Aphidius colemani Viereck (Hymenoptera: Aphidiidae) in the Middle Delta Egypt. Egyptian Journal of Biological Pest Control 14: 213-216.

Stanković S.S., Petrović A., Ilić Milošević M., Starý P., Kavallieratos N.G., Žikić V., Tomanović Ž. 2015. Morphological and molecular characterization of the common European Adialytus species (Hymenoptera: Braconidae: Aphidiinae) based on the mtCOI barcoding gene and geometric morphometrics of forewings. European Journal of Entomology 112: 165-174.

https://doi.org/10.14411/eje.2015.007

Starý P. 1969a. Aphids-ant-parasite relationship in Iraq. Insect Sociaux 4: 269-278.

https://doi.org/10.1007/BF02308999 
Starý P. 1969b. Aphids and their parasites associated with Oaks in Iraq. Proceeding of the Entomological Society of Washington 71 (3): 279-298.

Starý P. 1969c. Aphid migration and impact of an indigenous parasite Aphidius transcaspicus Telenga, on populations of Hyalopterus pruni (Geoffr.) in Iraq (Homoptera: Aphididae and Hymenoptera: Aphidiidae). Bulltein of the Entomological Society of Egypt 53: 185-198.

Starý P. 1970. Biology of Aphid Parasites (Hymenoptera: Aphidiidae) with Respect to Integrated Control. Dr W. Junk b.v., The Hague.

Starý P. 1974. Parasite spectrum (Hym.: Aphidiidae) of the green peach aphid Myzus persicae (Sulz.) (Hom., Aphididae). Bollettino del Laboratorio di Entomologia Agraria di Portici 31: 61-98.

Starý P. 1975. Aphidius colemani Viereck: its taxonomy, distribution and host range (Hymenoptera: Aphidiidae). Acta Entomologica Bohemoslovaca 72: 156-163.

Starý P. 1976. Aphid Parasites (Hymenoptera: Aphidiidae) of the Mediterranean Area. Dr. W. Junk b.v., The Hague /Academia, Prague.

Starý P. 1979. Aphid Parasites (Hymenoptera: Aphidiidae) of the Central Asian Area. Dr. W. Junk b.v., The Hague/Academia, Prague.

Starý P. 1981a. On the strategy, tactics and trends of host specificity evolution in aphid parasitoids (Hymenoptera: Aphidiidae). Acta Entomologica Bohemoslovaca 78: 65-75.

Starý P. 1981b. Biosystematic synopsis of parasitoids on cereal aphids in the western Palaearctic (Hymenoptera Aphidiidae; Homoptera Aphidoidea). Acta Entomologica Bohemoslovaca 78(6): 382396.

Starý P. \& Erdelen C. 1982. Aphid parasitoids [Hym.: Aphidiidae: Aphelinidae] from the Yemen Arab Republic. BioControl 27 (1): 105-108. https://doi.org/10.1007/BF02371943

Starý P. \& González D. 1978. Parasitoid spectrum of Acyrthosiphon aphids in Central Asia (Hymenoptera: Aphidiidae). Scandinavian Entomology 9: 140-145. https://doi.org/10.1163/187631278X00089

Starý P. \& Juchnevič L.A. 1978. Aphid parasites (Hymenoptera: Aphidiidae) from Kazakhstan USSR. Polskie Pismo Entomologiczne 48: 523-532.

Starý P. \& Kaddou I.K. 1971. Fauna and distribution of aphid parasites (Hym.: Aphidiidae) in Iraq. Acta Faunistica Entomologica Musei Nationalis Pragae 14: 179-197.

Starý P. \& Leclant F. 1977. Pauesia (Pauesia) cedrobii, n.sp., premier parasite connu du puceron de cedre Cedrobium laportei Remaudière (Hym.: Aphidiidae). Annales de la Société entomologique de France 13 (1): 159-163.

Starý P. \& Sekkat A. 1987. Parasitoids (Hymenoptera: Aphidiinae) of aphid pests in Morocco. Annales de la Société entomologique de France 23 (3): 145-149.

Starý P., González D. \& Hall J.C. 1980. Aphidius eadyi n. sp. (Hymenoptera: Aphidiidae), a widely distributed parasitoid of the pea aphid Acyrthosiphon pisum (Harris) in the Palearctic. Entomologia Scandinavica 11: 473-480. https://doi.org/10.1163/187631280794710079

Starý P., Remaudière G., González D. \& Shahrokhi S. 2000. A review and host associations of aphid parasitoids (Hym.: Braconidae: Aphidiinae) of Iran. Parasitica 56 (1): 15-41.

Starý P., Rakhshani E. \& Talebi A.A. 2005. Parasitoids of aphid pests on conifers and their state as biocontrol agents in the Middle East to Central Asia on the world background (Hym., Braconidae, Aphidiinae; Hom., Aphididae). Egyptian Journal of Biological Pest Control 15 (2): 147-151. 
Starý P., Rakhshani E., Tomanović Ž. Kavallieratos N.G. \& Havelka. J. 2013. Order Hymenoptera, family Braconidae - Aphidiinae Aphid parasitoids of the Arabian Peninnsula In: van Harten A. (ed.), Arthropod Fauna of UAE Vol 5: 407-425. Al Amal Printing Press. Abu Dhabi.

Starý P., Rakhshani E., Žikić V., Kavallieratos N.G., Lavandero B. \& Tomanović Ž. 2014. Altitudinal zonation of aphid parasitoids (Hymenoptera: Braconidae: Aphidiinae) in the Neotropical region. Entomological News 124 (2): 86-97. https://doi.org/10.3157/021.124.0203

Starý P., Remaudière G. \& Leclant F. 1971. Les Aphidiidae (Hym.) de France et leurs hotes (Hom.: Aphididae). Entomophaga 5: 1-65.

Taheri S. \& Rakhshani E. 2013. Identification of aphid parasitoids (Hym.: Braconidae: Aphidiinae) and determination of their host relationships in Southern Zagros. Journal of Plant Protection (Ferdowsi University Iran) 27 (1): 85-95. [In Persian.]

Talebi A.A., Rakhshani E., Fathipour Y., Starý P., Tomanović Ž. \& Rajabi-Mazhar N. 2009. Aphids and their parasitoids (Hym.: Braconidae: Aphidiinae) associated with medicinal plants in Iran. AmericanEurasian Journal of Sustainable Agriculture 3 (2): 205-219.

Talhouk A.S. 1961. Records of entomophagous insects from Lebanon. Entomophaga 6: 207-209.

Tomanović Ž., Kavallieratos N.G., Athanassiou C.G. \& Stanisavljević L.Ž. 2003. A review of the West Palaearctic aphidiines (Hymenoptera: Braconidae: Aphidiinae) parasitic on Uroleucon spp., with the description of a new species. Annales de la Société entomologique de France (N.S.) 39 (4): 343-353.

Tomanović Ž., Rakhshani E., Starý P., Kavallieratos N.G., Stanisavljević L.Ž., Žikić V. \& Athanassiou C.G. 2007. Phylogenetic relationships between the genera Aphidius Nees and Lysaphidus (Hymenoptera: Braconidae: Aphidiinae) with description of Aphidius iranicus sp. nov. The Canadian Entomologist 139: 297-307. https://doi.org/10.4039/n06-007

Tomanović Ž., Beyerslan A., Erdoğan Ö.Ç. \& Žikić Ž. 2008. New records of aphid parasitoids (Hymenoptera: Braconidae: Aphidiinae) from Turkey. Periodicum Biologorum 110 (4): 335-338.

Tomanović Ž., Starý P., Kavallieratos N.G., Gagić V., Plećaš M., Janković M., Rakhshani E., Ćetković A. \& Petrović A. 2012. Aphid parasitoids (Hymenoptera: Braconidae: Aphidiinae) in wetland habitats in western Palaearctic: key and associated aphid parasitoid guilds. Annales de la Société entomologique de France (N.S.) 48 (1-2): 189-198. https://doi.org/10.1080/00379271.2012.10697763

Tomanović Ž., Petrović A., Mitrović M., Kavallieratos N.G., Starý P., Rakhshani E., Rakhshanipour M., Popović A., Shukshuk A.H \& Ivanović A. 2014. Molecular and morphological variability within the Aphidius colemani group with redescription of Aphidius platensis Brethes (Hymenoptera: Braconidae: Aphidiinae). Bulletin of Entomological Research, 104, 552-565.

https://doi.org/10.1017/S0007485314000327

Tomanović Ž., Mitrović M., Petrović A., Kavallieratos N.G., Zikić V., Ivanović A., Rakhshani E., Starý P. \& Vorburger C. 2018. Revision of the European Lysiphlebus species (Hymenoptera: Braconidae: Aphidiinae) on the basis of COI and 28SD2 molecular markers and morphology. Arthropod Systematics \& Phylogeny 76 (2): 179-213.

Tozlu G., Gültekin L., Hayat R. \& Güçlü Ş. 2002. Studies on the natural enemies of cabbage pests in Erzurum. In: Özbek H., Güçlü Ş. and Hayat R. (eds) Proceedings of the $5^{\text {th }}$ Turkish National Congress of Biological Control: 227-235. Ataturk University, Erzurum.

Tremblay E. \& Eady R.D. 1978. Lysiphlebus confusus n.sp. per Lysiphlebus ambiguus sensu auct. nec Haliday (1834) (Hymenoptera: Ichneumonoidea). Bollettino del Laboratorio di entomologia Agraria Filippo Silvestri Portici 35: 180-184. 
Tremblay E., Kawar N. \& Barbagallo S. 1985. Aphids (Homoptera: Aphidoidea) and aphidiines (Hymenoptera: Braconidae) of Lebanon. Bollettino del Laboratorio di entomologia Agraria Filippo Silvestri Portici 42: 19-32.

Uysal M., Starý P., Sahbaz A. \& Özsemerci F. 2004. A review of aphid parasitoids (Hymenoptera: Braconidae: Aphidiinae) of Turkey. Egyptian Journal of Biological Pest Control 12 (2): 355-370.

van den Bosch R. 1957. The spotted alfalfa and its parasites in Mediterranean region, Middle East, and East Africa. Journal of Economic Entomology 50: 352-356. https://doi.org/10.1093/jee/50.3.352

van den Bosch R. \& Messenger P.S. 1973. Biological Control. Intext Educational Publishers, New York/ London.

van den Bosch R., Frazer B.D., Davis C.S., Messenger P.S. \& Hom R. 1970. Trioxys pallidus - an effective new walnut aphid parasite from Iran. California Agriculture 24 (11): 8-10.

Vollhardt I.M.G., Tscharntke T., Wäckers F.L., Bianchi F.J.J.A. \& Thies C. 2008. Diversity of cereal aphid parasitoids in simple and complex landscapes. Agriculture Ecosystems \& Environment 126: 289292. https://doi.org/10.1016/j.agee.2008.01.024

Wool D. \& Burstein M. 1991. Parasitoids of the gall-forming aphid Smynthurodes betae [Aphidoidea: Fordinae] in Israel. BioControl 36 (4): 531-538.

Yoldaş Z., Öncuer C. \& Karsavuran Y. 1990. Les ennemies naturel déterminés dans les cultures de tomates industrielles. In: Proceedings of the $2^{\text {nd }}$ Turkish National Congress of Biological Control: 189196. Ege Universitesi, Izmir.

Yoldaş Z., Gülcük M., Turanli D. \& Madanlar N. 2002. Mass rearing of effective biological control agents against aphids injurious to vegetables grown in greenhouses. In: Özbek H., Güçlü Ş. and Hayat R. (eds) Proceedings of $5^{\text {th }}$ Turkish National Congress of Biological Control: 199-207. Ataturk University, Erzurum.

Yoldaş Z., Güncan A. \& Koçlu T. 2011. Seasonal occurrence of aphids and their natural enemies in Satsuma mandarin orchards in Izmir Turkey. Turkish Journal of Entomology 35 (1): 59-74.

Yumruktepe R. \& Uygun N. 1994. Determination of aphid species (Homoptera: Aphididae) and their natural enemies in citrus orchards in Eastern Mediterranean region. In: Proceedings of the $3^{\text {rd }}$ Turkish National Congress of Biological Control: 1-12. Ege Universitesi, Izmir.

Zareh N., González D., Ahmadi A., Esmaili M., Maleki-Milani H., Vafabakhsh J., Gilstrap F., Starý P., Woolley J.B. \& Thompson F.C. 1995. A search for the Russian wheat aphid Diuraphis noxia and its natural enemies in Iran. In: Proceedings of the 12 ${ }^{\text {th }}$ Iranian Plant Protection Congress Vol. 1: 12. University of Tehran, Karadj.

Zeren O. \& Düzgüneş Z. 1983. A Study on identifcation, host plants, effect on host plant and natural enemies of aphids, causing damage on vegetables in Cukurova region. No. 59. Turkish Ministry of Agriculture Research Serie Ankara. [In Turkish.]

Žikić V., Lazarević M. \& Milošević D. 2017. Host range patterning of parasitoid wasps Aphidiinae (Hymenoptera: Braconidae). Zoologischer Anzeiger 268: 75-83.

https://doi.org/10.1016/j.jcz.2016.10.001

Žikić V., Stanković S.S., Ilić-Milošević M., Petrović-Obradović O., Petrović A., Starý P. \& Tomanović Ž. 2015. First detection of Lysiphlebus testaceipes (Cresson) (Hymenoptera: Aphidiinae) in Serbia: an introduced species invading Europe. North-Western Journal of Zoology 11 (1): 97-101. 
Žikić V., Tomanović Ž., Ivanović A., Kavallieratos N.G., Starý P., Stanisavljević L.Ž. \& Rakhshani E. 2009. Morphological characterization of Ephedrus persicae biotypes (Hymenoptera: Braconidae: Aphidiinae) in the Palaearctic. Annals of the Entomological Society of America 102 (1): 1-11. https://doi.org/10.1603/008.102.0101

Manuscript received: $1^{\text {rst }}$ November 2018

Manuscript accepted: 26 May 2019

Published on: 16 September 2019

Topic editor: Gavin Broad

Desk editor: Marianne Salaün

Printed versions of all papers are also deposited in the libraries of the institutes that are members of the EJT consortium: Muséum national d'Histoire naturelle, Paris, France; Botanic Garden Meise, Belgium; Royal Museum for Central Africa, Tervuren, Belgium; Royal Belgian Institute of Natural Sciences, Brussels, Belgium; Natural History Museum of Denmark, Copenhagen, Denmark; Naturalis Biodiversity Center, Leiden, the Netherlands; Museo Nacional de Ciencias Naturales-CSIC, Madrid, Spain; Real Jardín Botánico de Madrid CSIC, Spain; Zoological Research Museum Alexander Koenig, Bonn, Germany; National Museum, Prague, Czech Republic. 


\section{(3) \\ - I.}

(3)

$150 \mu \mathrm{m}$

150

1)

$150 \mu \mathrm{m}$

the

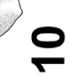

1.1.2.

3

कृ

$\dot{0}+\frac{2}{3}$

$\dot{3}$

:

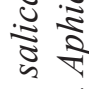

空

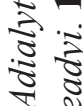

3र

was

ค.

竎:

$\longrightarrow+150 \mathrm{~m}$

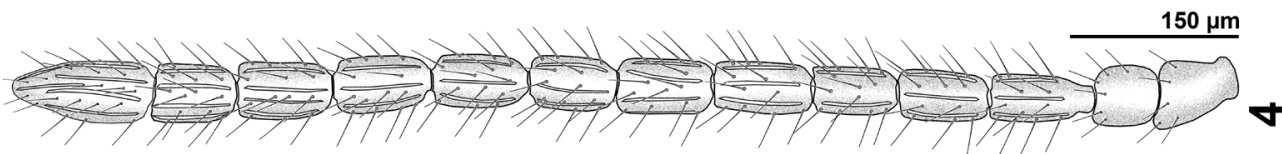

(3)

$150 \mu \mathrm{m}$

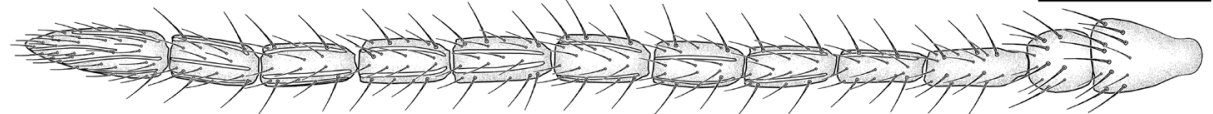

$150 \mu \mathrm{m}$
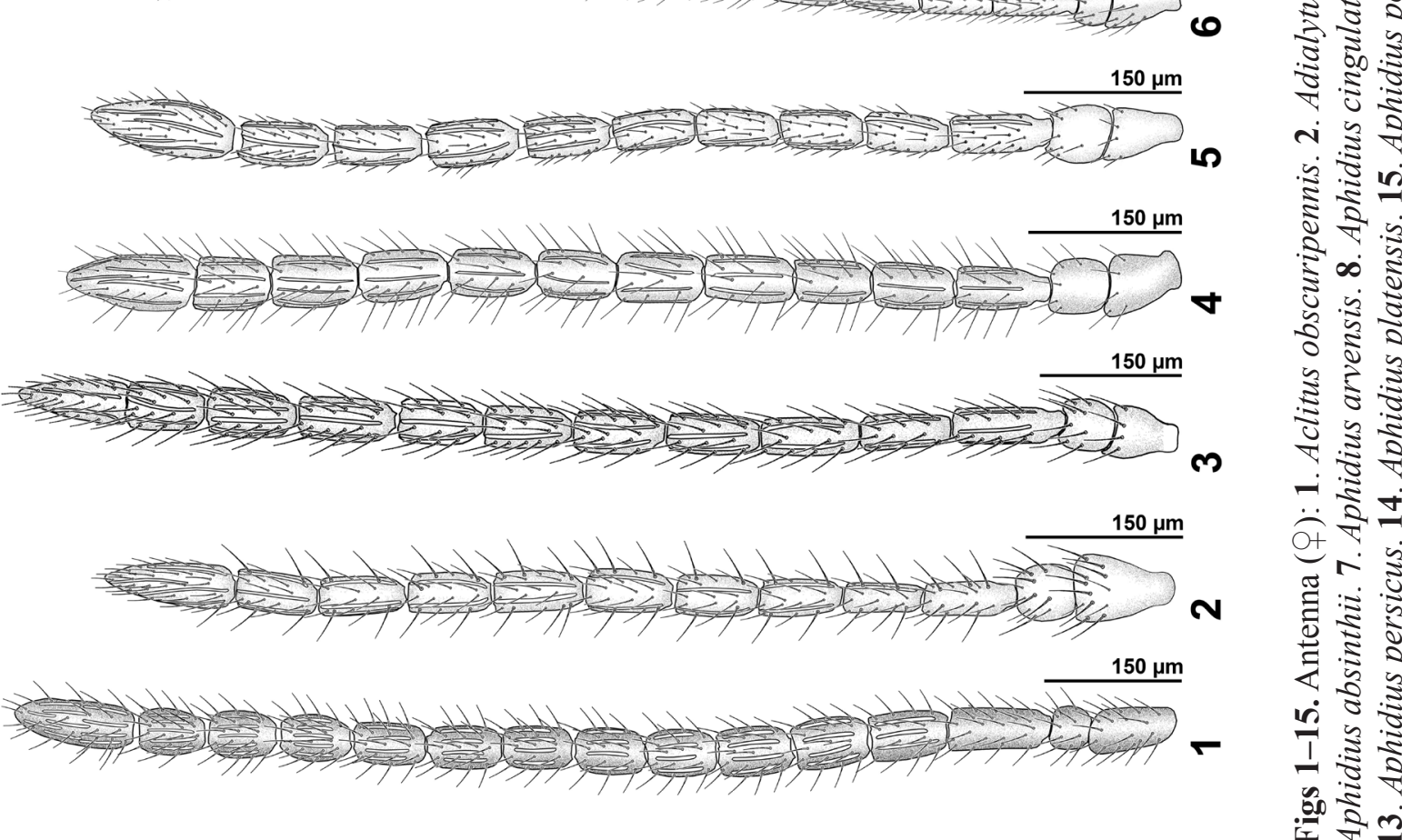


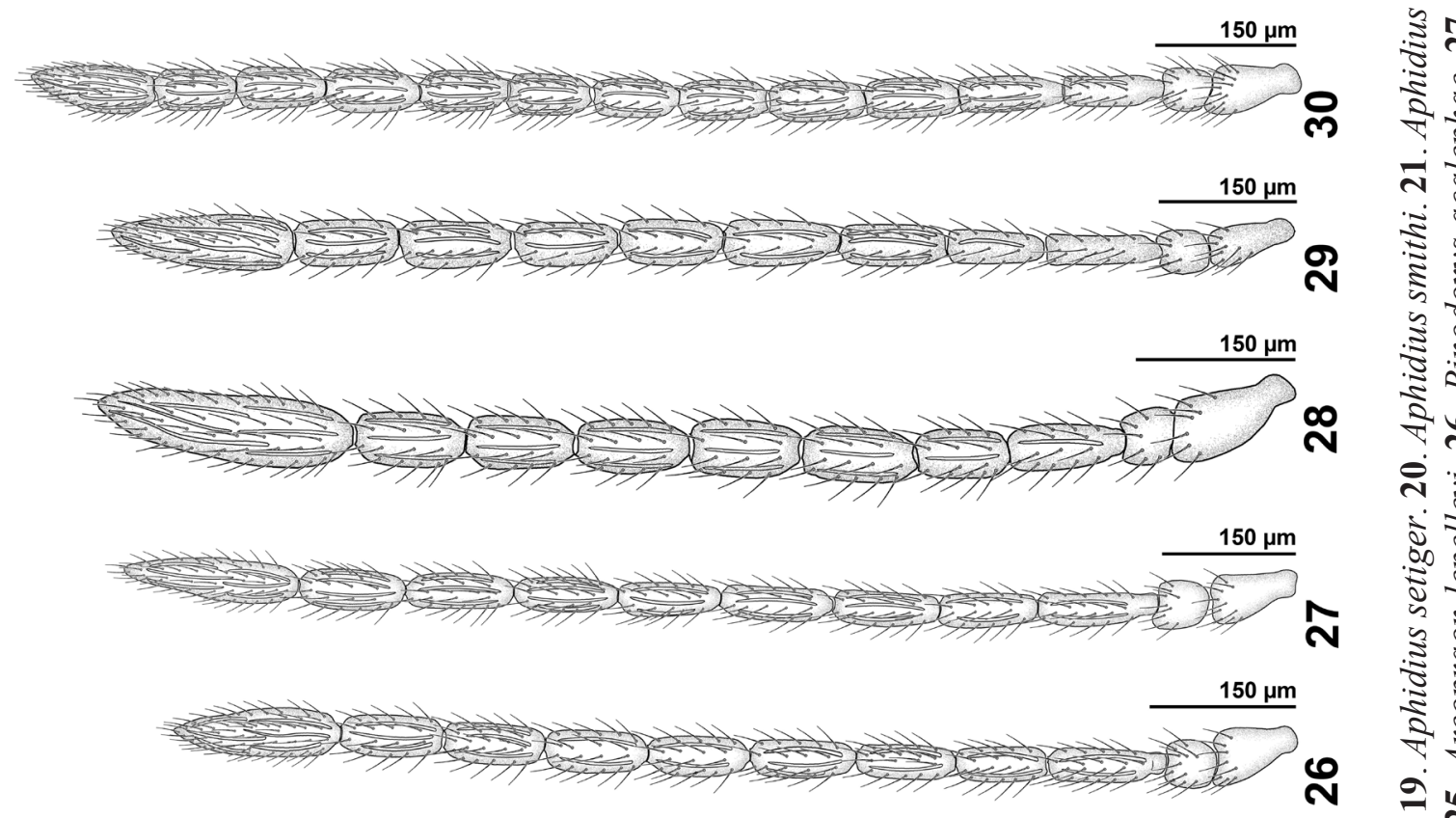

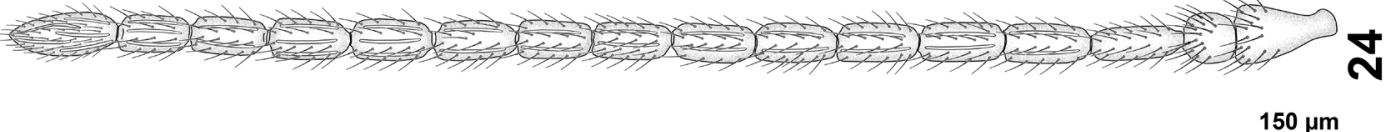

(2) 


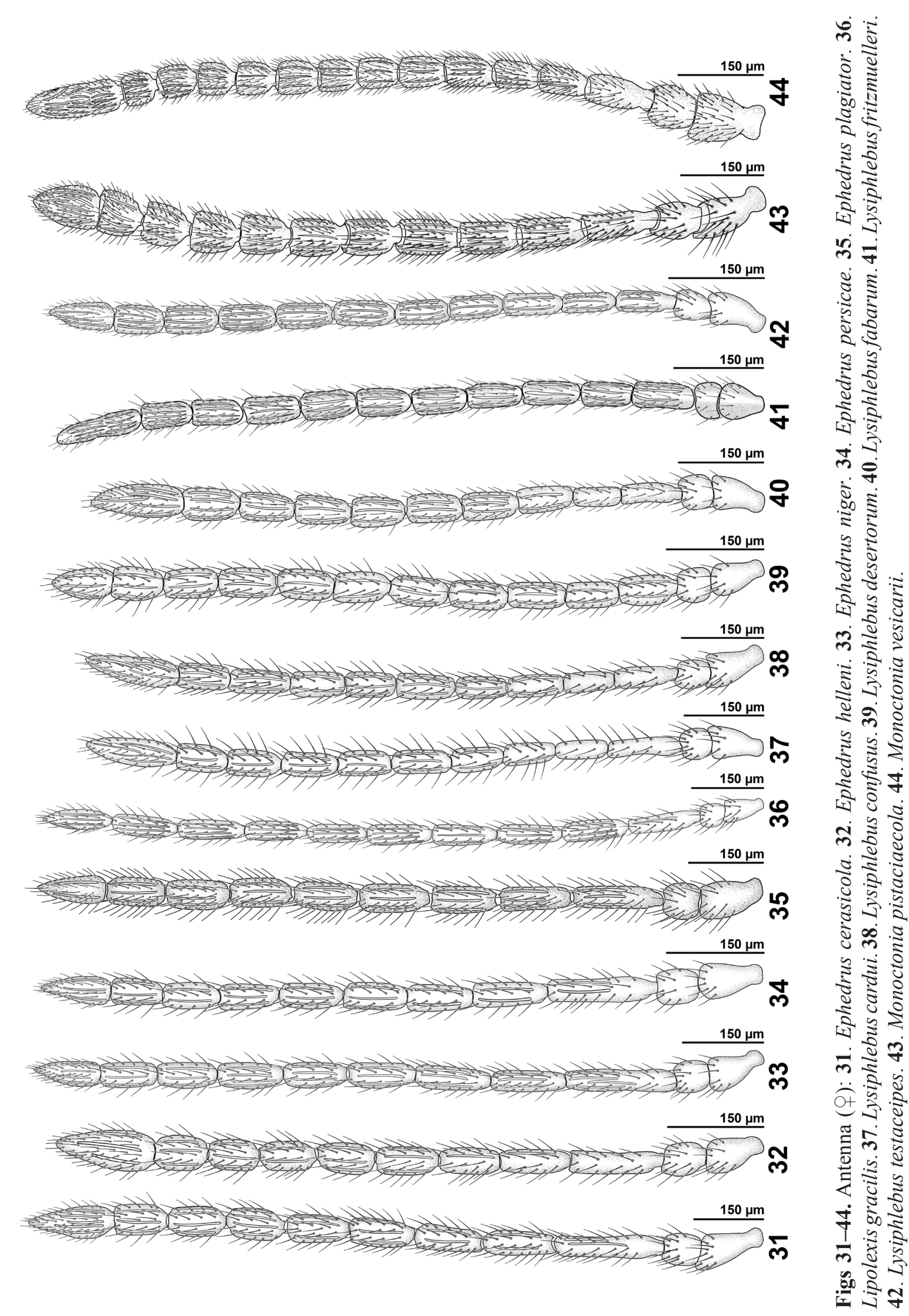




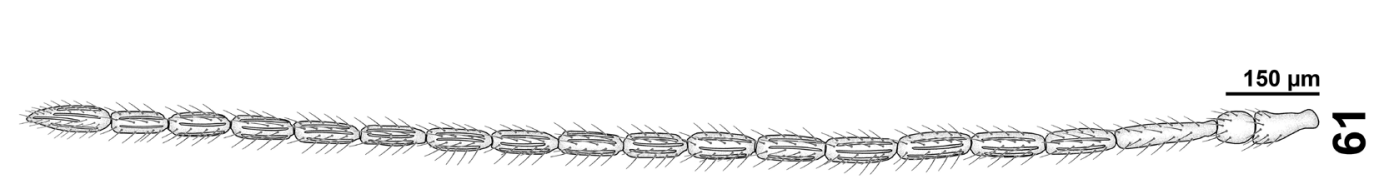

in

竞

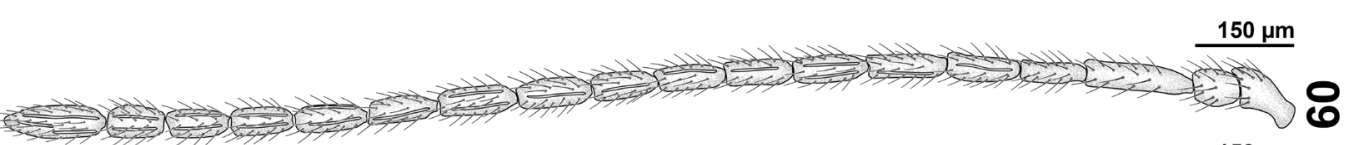

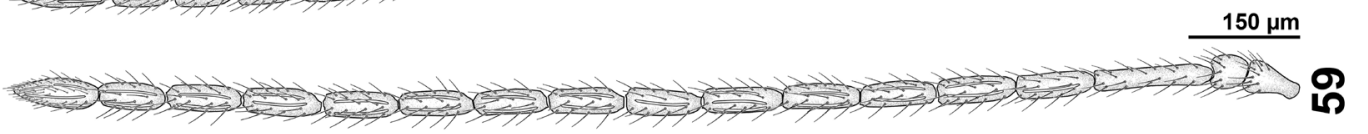

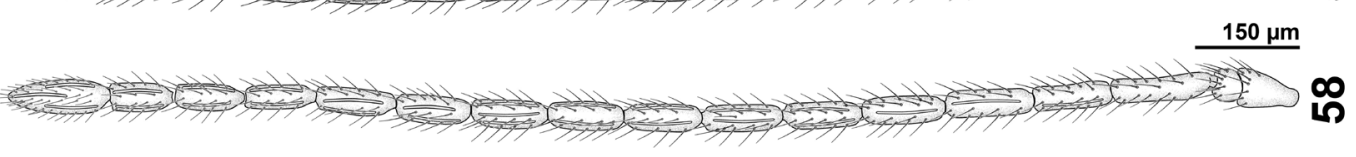

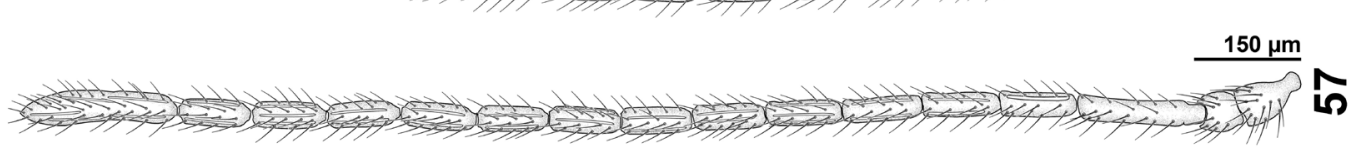

10

-

2.

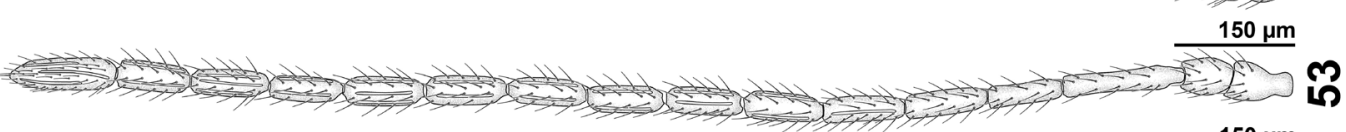

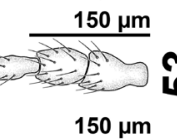

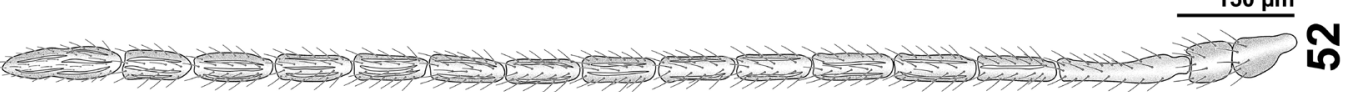

in

से

$\Rightarrow+150 \mathrm{\mu m}$ O

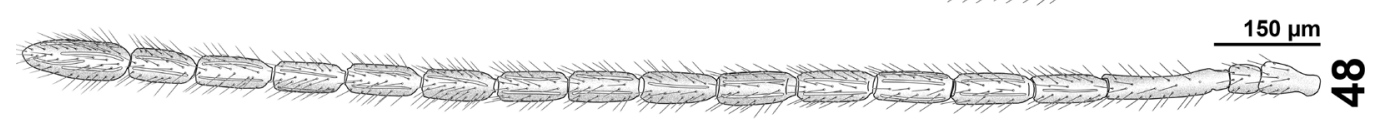

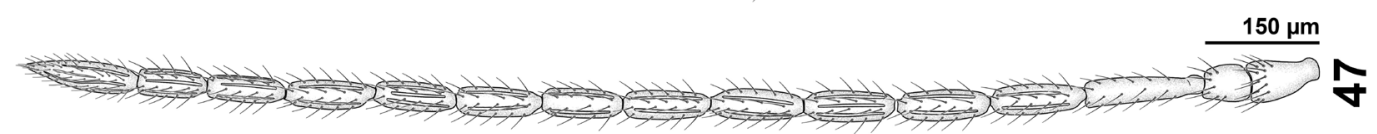

(3) Stw






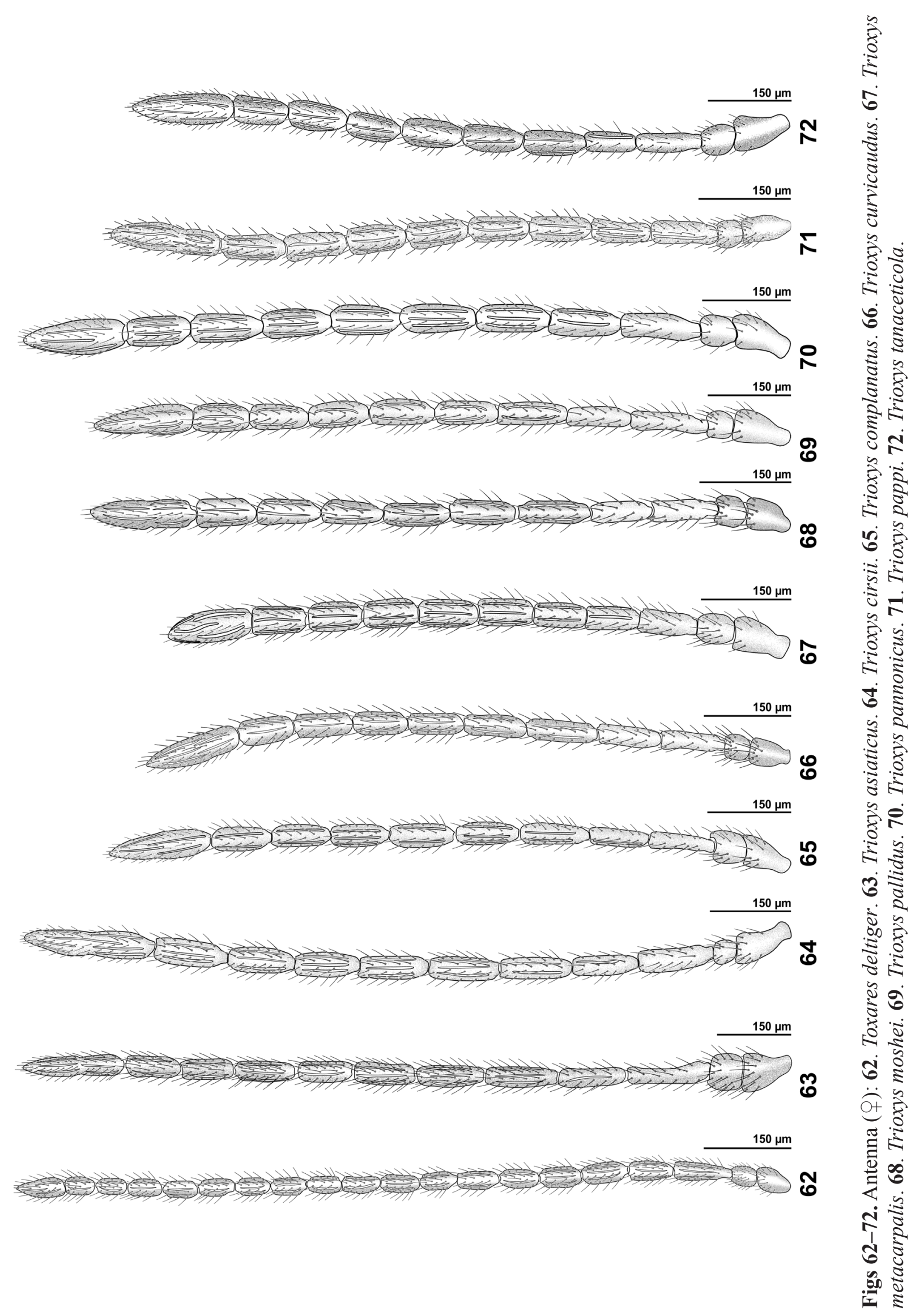




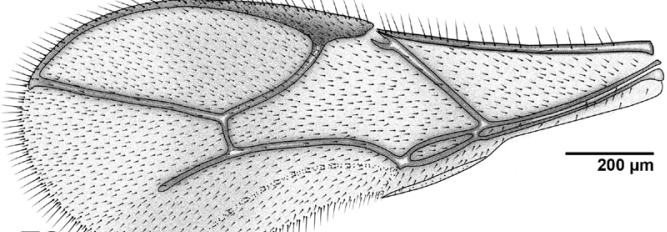

73

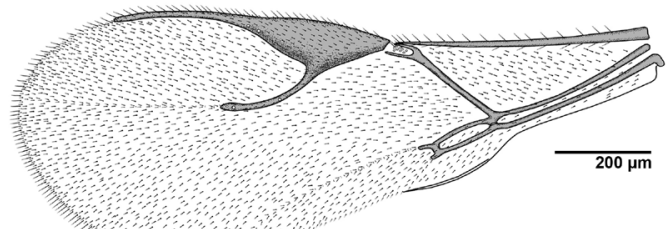

75
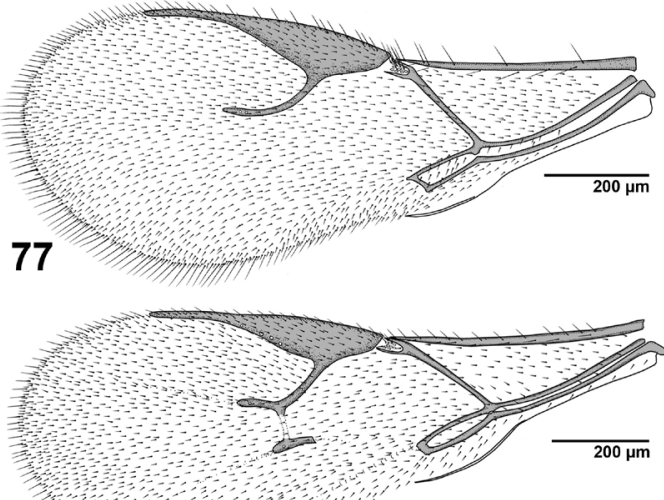

79
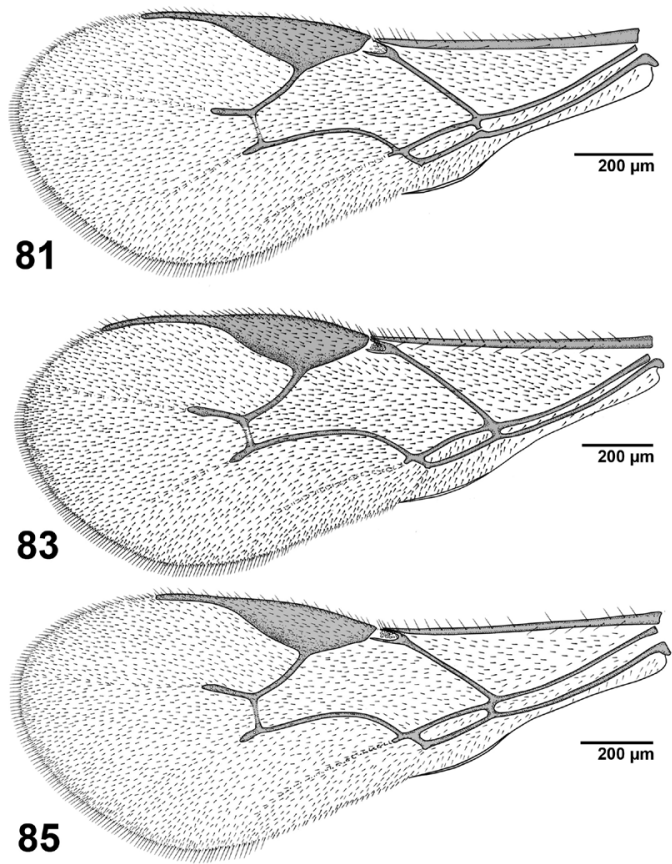
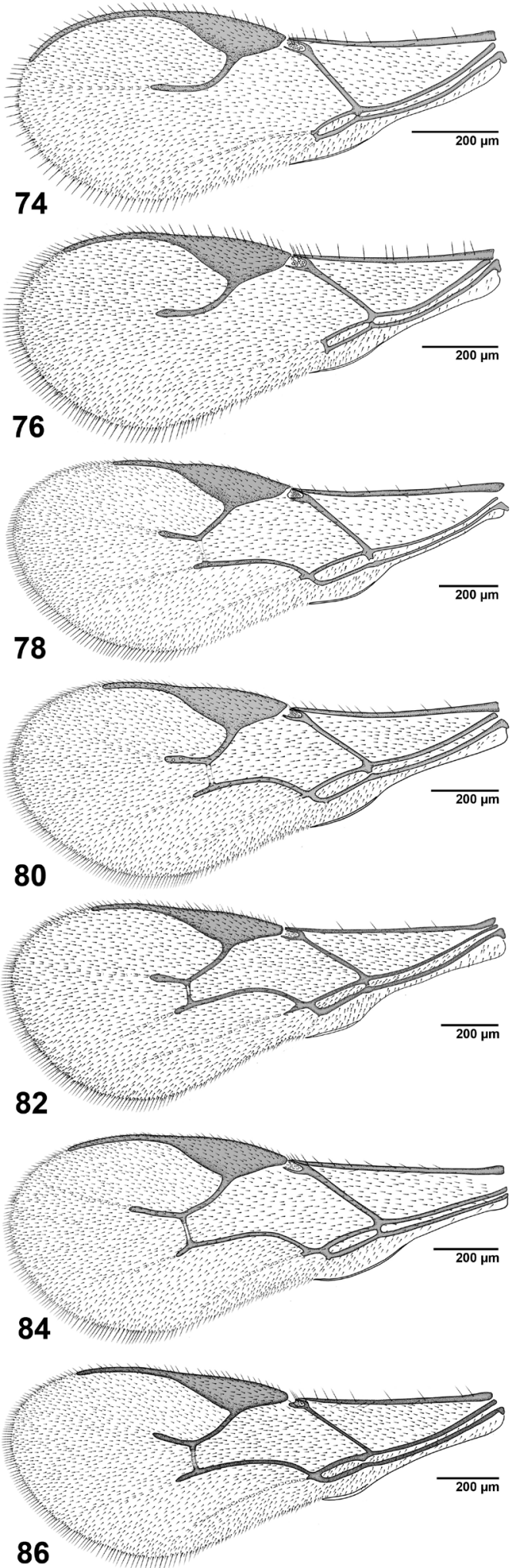

Figs 73-86. Forewing ( $($ ): 73. Aclitus obscuripennis. 74. Adialytus ambiguus. 75. Adialytus salicaphis. 76. Adialytus thelaxis. 77. Adialytus veronicaecola. 78. Aphidius absinthii. 79. Aphidius arvensis. 80. Aphidius asteris. 81. Aphidius avenae. 82. Aphidius banksae. 83. Aphidius cingulatus. 84. Aphidius colemani. 85. Aphidius eadyi. 86. Aphidius eglanteriae. 

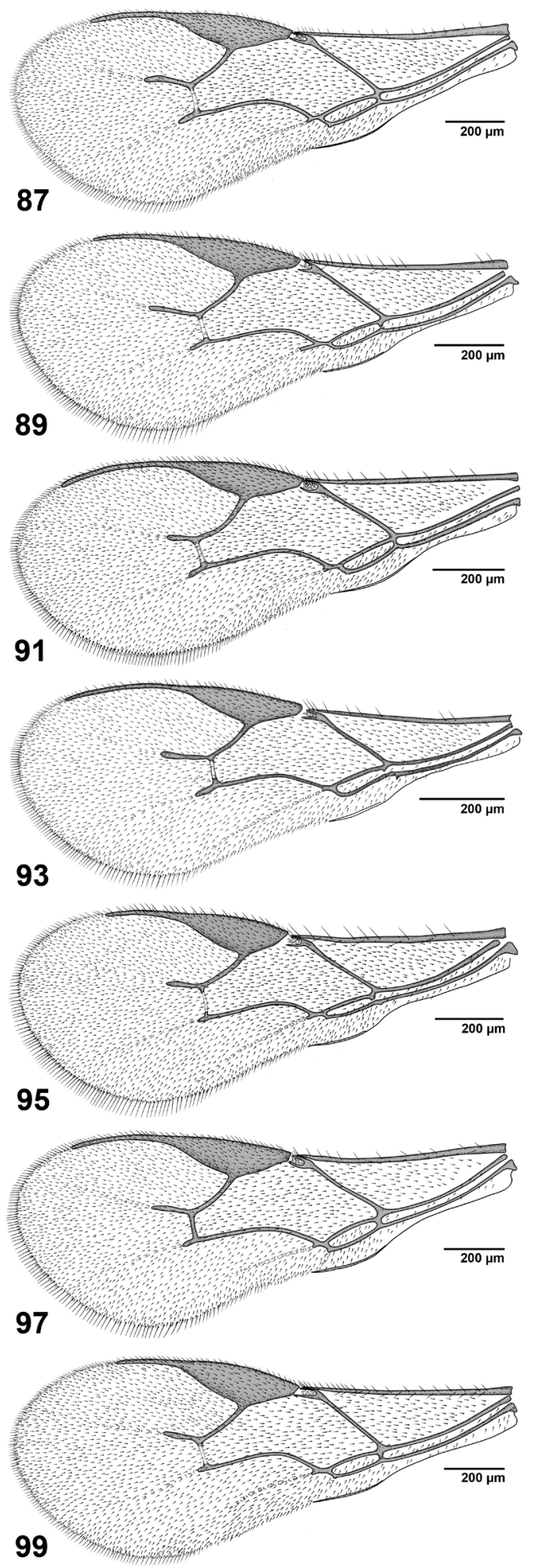
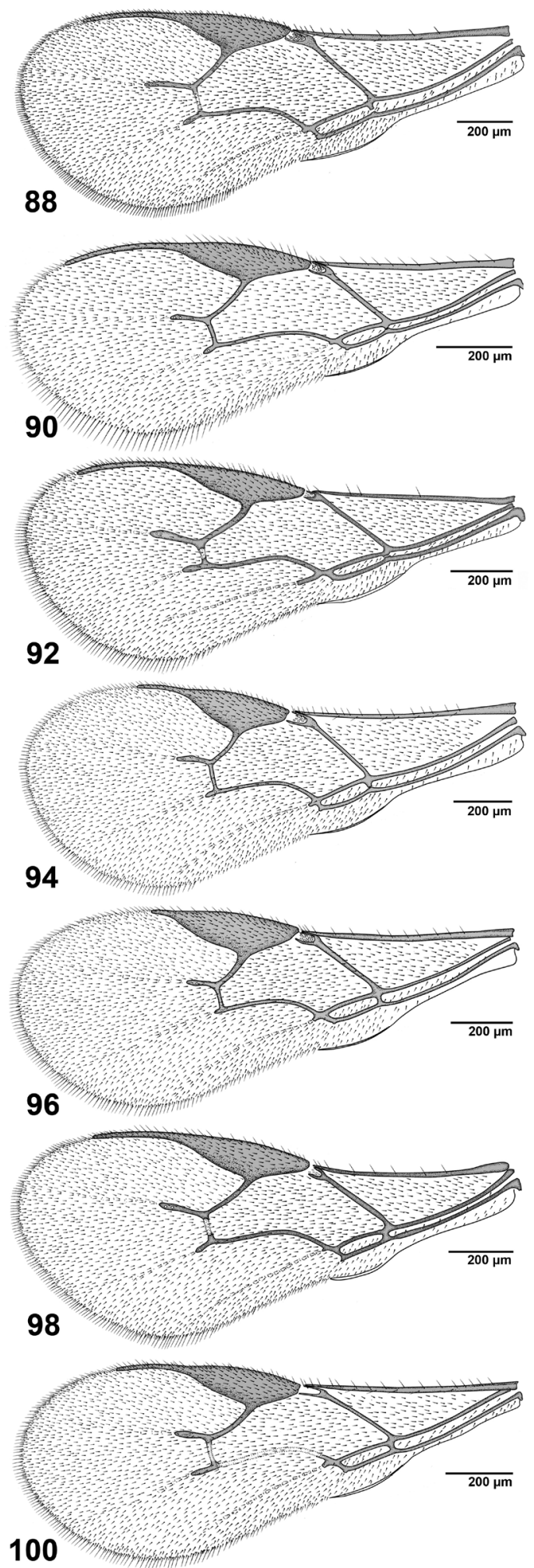

Figs 87-100. Forewing ( + ): 87. Aphidius ervi. 88. Aphidius funebris. 89. Aphidius hieraciorum. 90. Aphidius iranicus. 91. Aphidius matricariae. 92. Aphidius microlophii. 93. Aphidius myzocallidis. 94. Aphidius persicus. 95. Aphidius platensis. 96. Aphidius popovi. 97. Aphidius rhopalosiphi. 98. Aphidius ribis. 99. Aphidius rosae. 100. Aphidius salicis. 

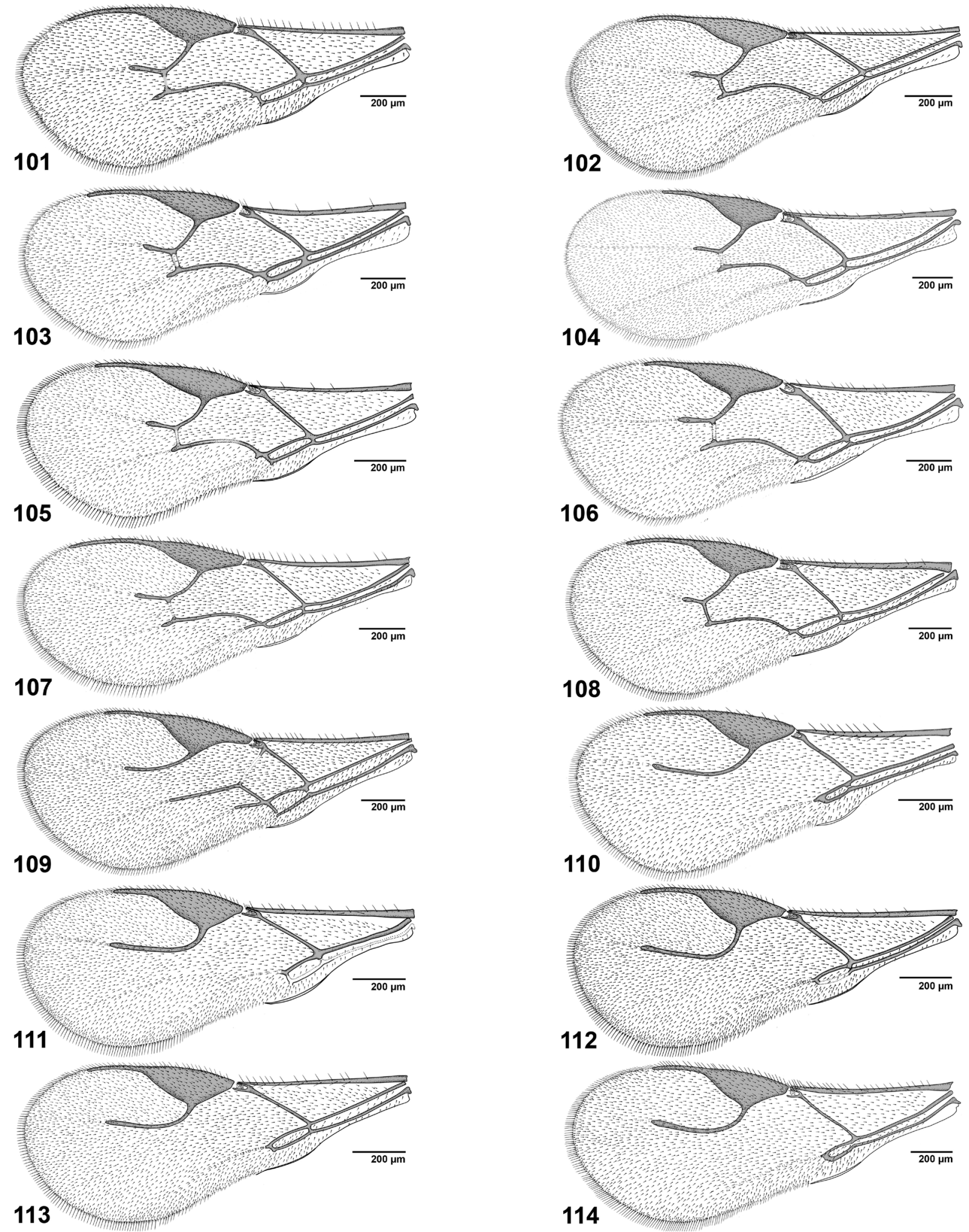

Figs 101-114. Forewing (): 101. Aphidius setiger. 102. Aphidius smithi. 103. Aphidius sonchi. 104. Aphidius stigmaticus. 105. Aphidius transcaspicus. 106. Aphidius uroleuci. 107. Aphidius urticae. 108. Aphidius uzbekistanicus. 109. Areopraon lepelleyi. 110. Betuloxys hortorum. 111. Binodoxys acalephae. 112. Binodoxys angelicae. 113. Binodoxys brevicornis. 114. Binodoxys centaureae. 

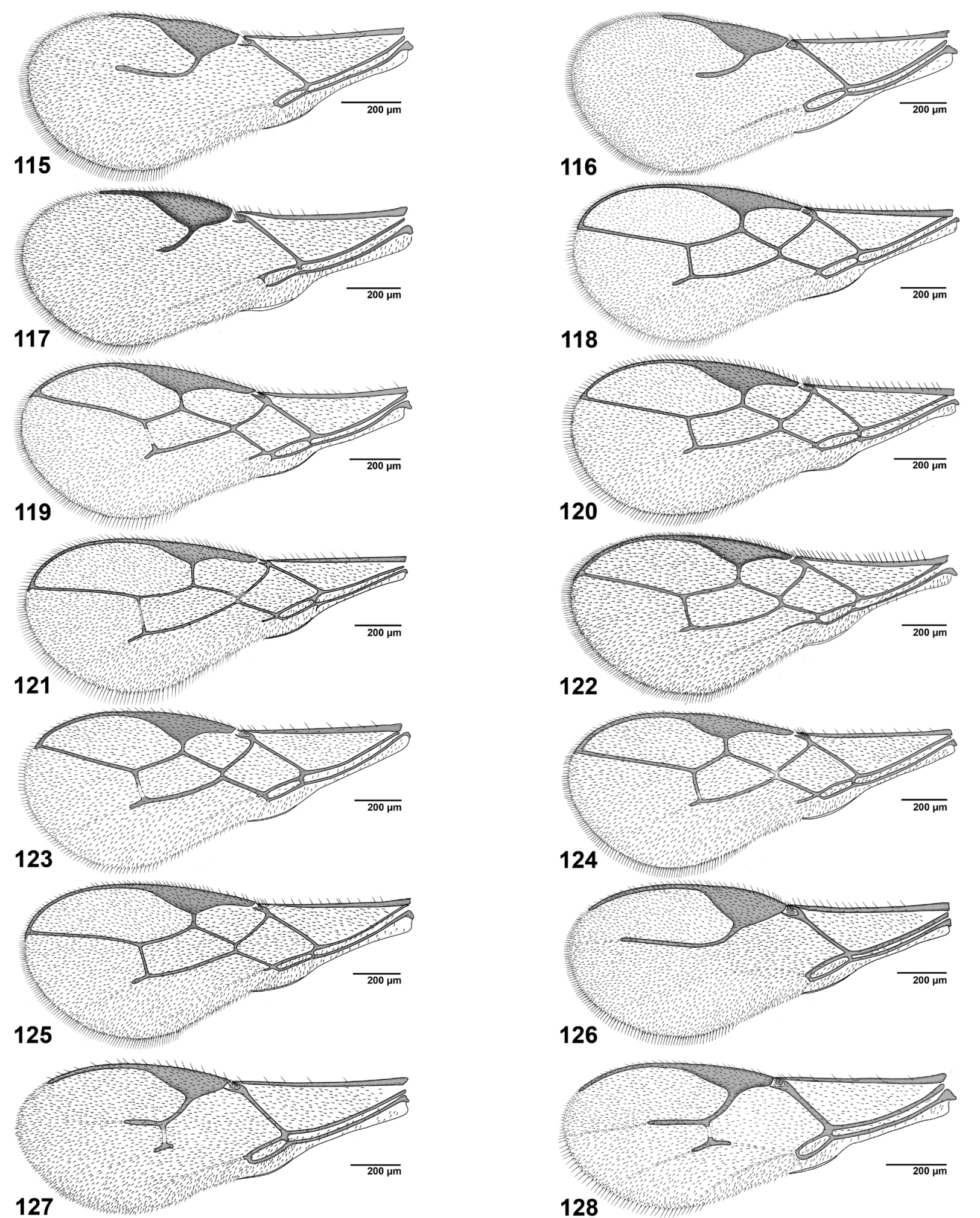

Figs 115-128. Forewing(): 115. Binodoxys heraclei.116. Diaeretiella rapae. 117. Diaeretus leucopterus. 118. Ephedrus cerasicola. 119. Ephedrus chaitophori. 120. Ephedrus helleni. 121. Ephedrus lacertosus. 122. Ephedrus nacheri. 123. Ephedrus niger. 124. Ephedrus persicae. 125. Ephedrus plagiator. 126. Lipolexis gracilis. 127. Lysiphlebus cardui. 128. Lysiphlebus confusus. 



Figs 129-142. Forewing ( $($ )): 129. Lysiphlebus desertorum. 130. Lysiphlebus fabarum. 131. Lysiphlebus fritzmuelleri. 132. Lysiphlebus testaceipes. 133. Monoctonia pistaciaecola. 134. Monoctonia vesicarii. 135. Monoctonus crepidis. 136. Monoctonus mali. 137. Pauesia abietis. 138. Pauesia anatolica. 139. Pauesia antennata. 140. Pauesia cedrobii. 141. Pauesia hazratbalensis. 142. Pauesia picta. 

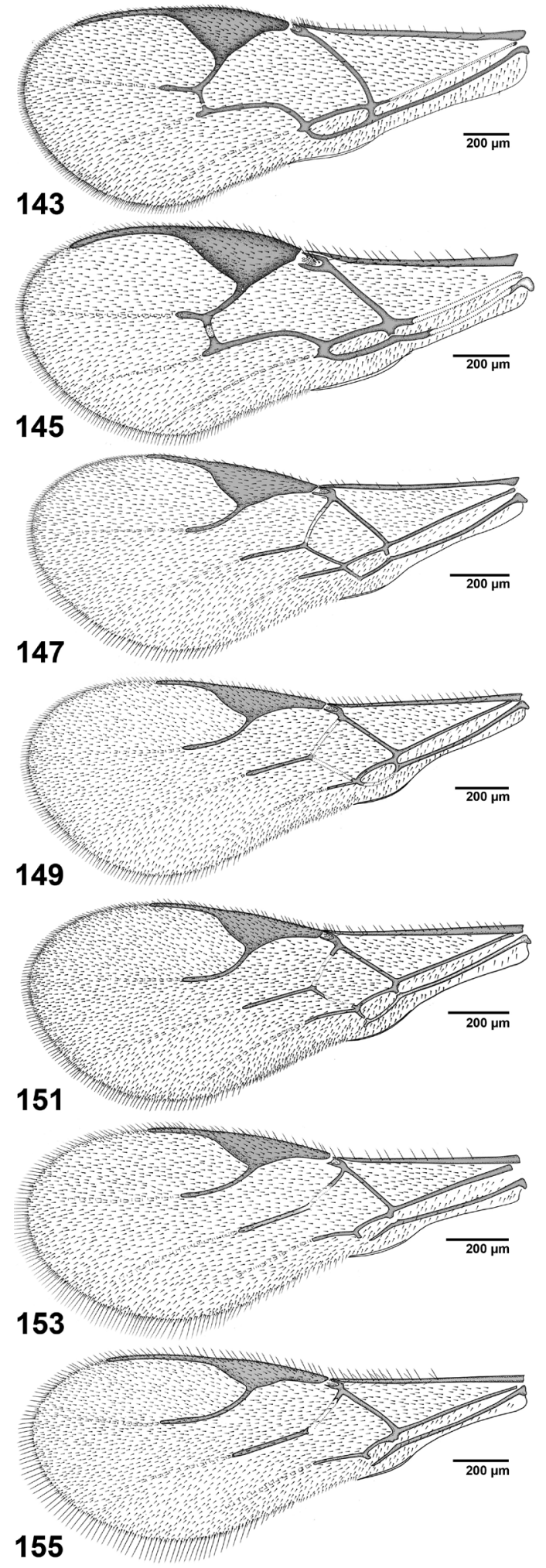
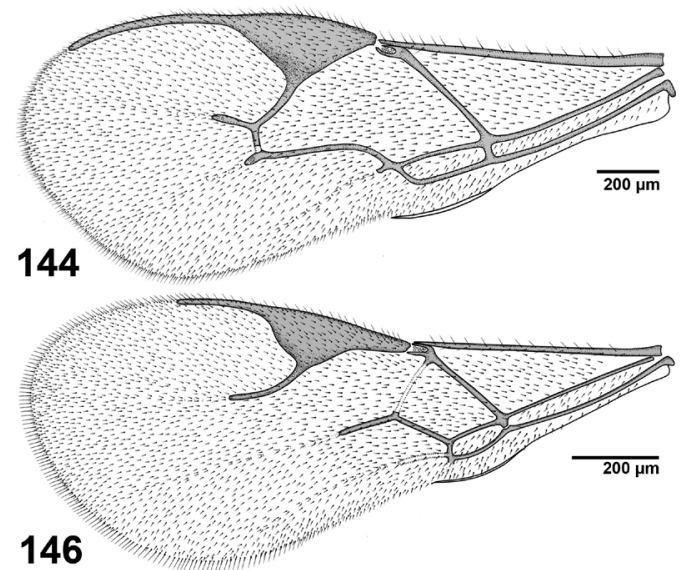

146
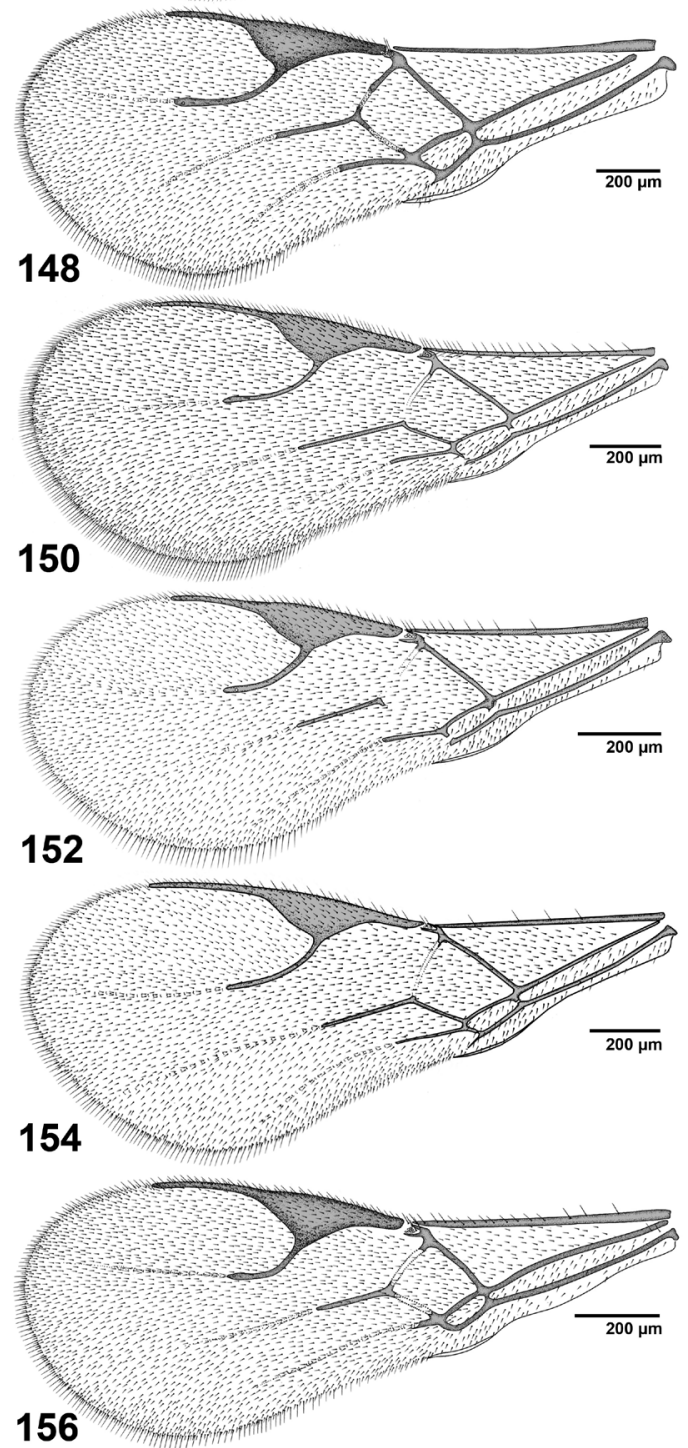

Figs 143-156. Forewing ( $९$ ): 143. Pauesia pini. 144. Pauesia silana. 145. Pauesia unilachni. 146. Praon abjectum. 147. Praon absinthii. 148. Praon athenaeum. 149. Praon barbatum. 150. Praon bicolor. 151. Praon exsoletum. 152. Praon flavinode. 153. Praon gallicum. 154. Praon longicorne. 155. Praon necans. 156. Praon nonveilleri. 

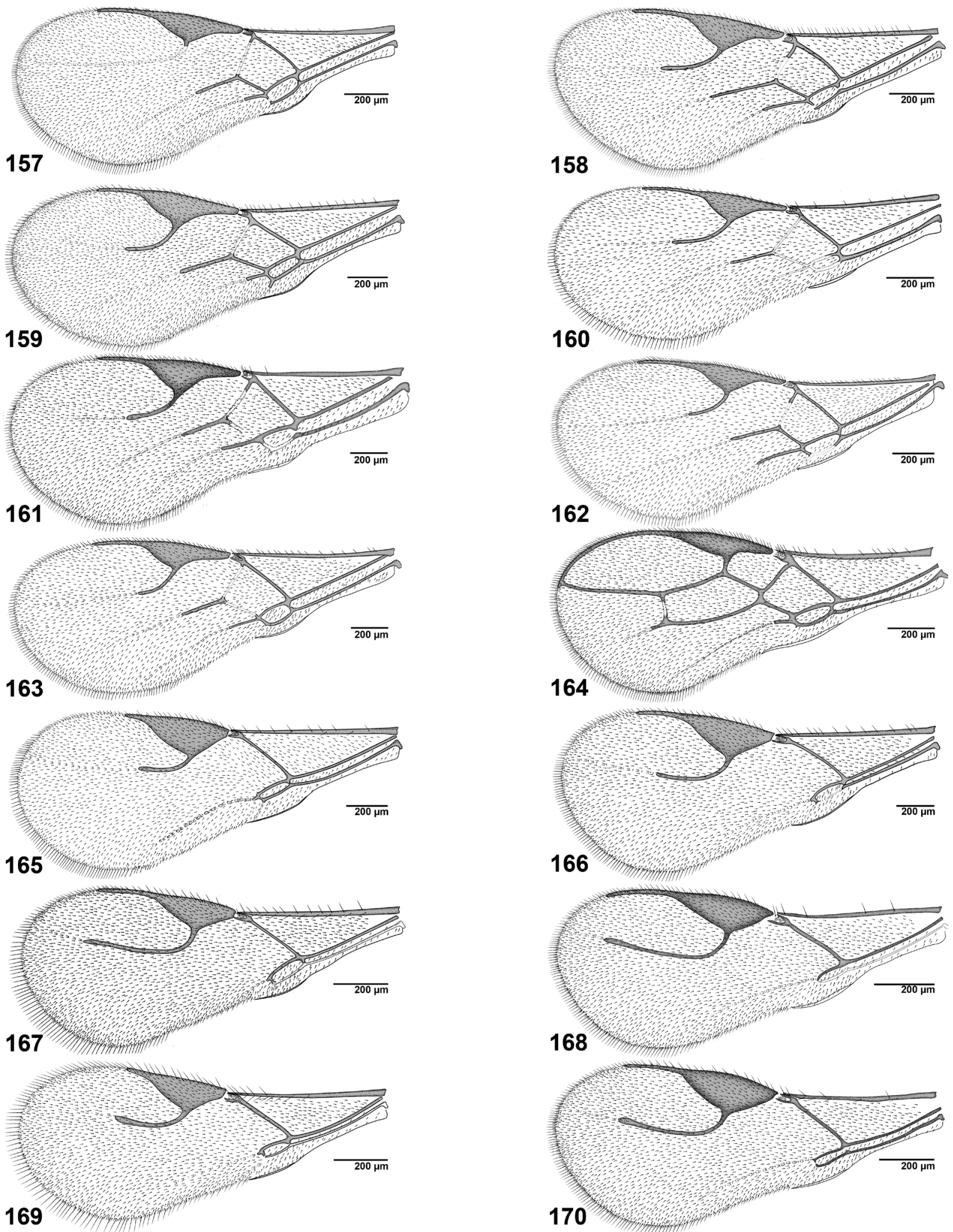

Figs 157-170. Forewing ( + ): 157. Praon orpheusi. 158. Praon pubescens. 159. Praon rosaecola. 160. Praon unitum. 161. Praon uroleucon. 162. Praon volucre. 163. Praon yomenae. 164. Toxares deltiger. 165. Trioxys asiaticus. 166. Trioxys cirsii. 167. Trioxys complanatus. 168. Trioxys curvicaudus. 169. Trioxys metacarpalis. 170. Trioxys moshei. 


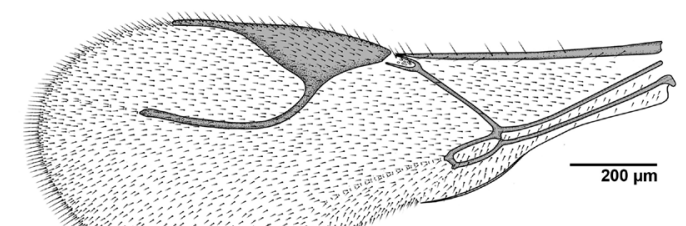

171

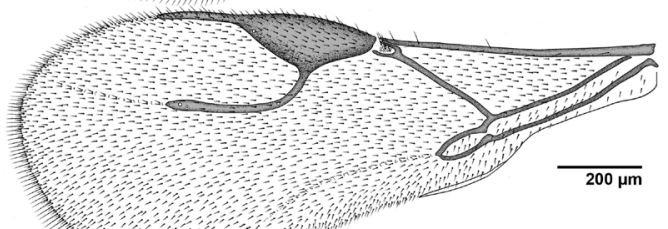

173

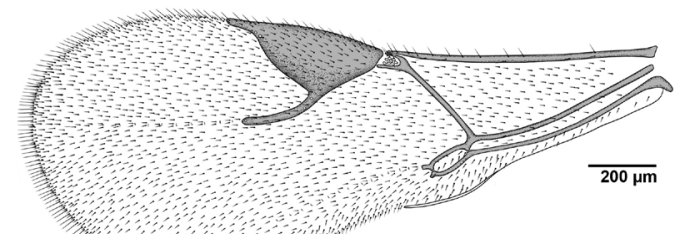

172

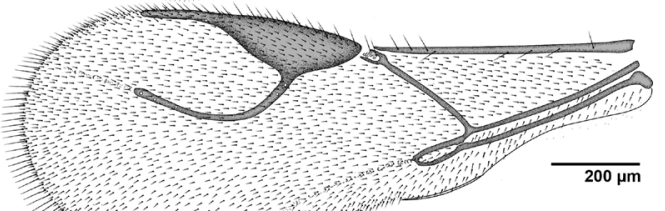

174

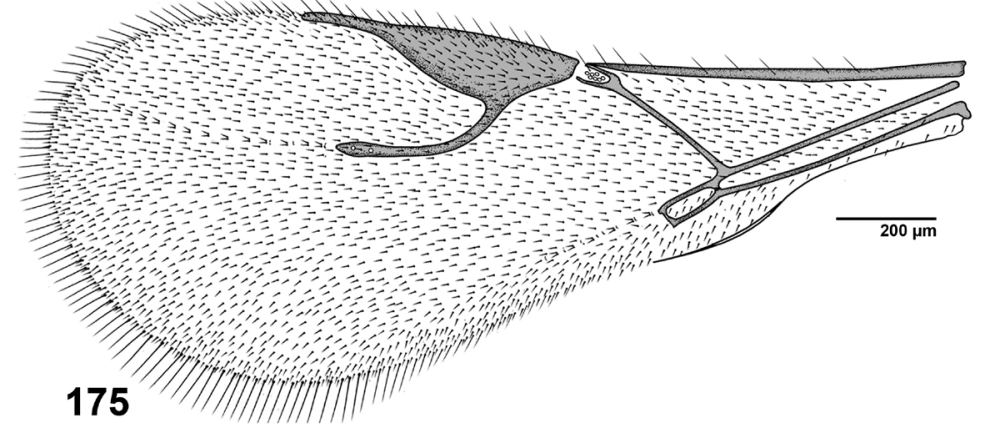

Figs 171-175. Forewing (): 171. Trioxys pallidus. 172. Trioxys pannonicus. 173. Trioxys pappi. 174. Trioxys quercicola. 175. Trioxys tanaceticola. 



Figs 176-191. Dorsal aspect of mesonotum (†): 176. Aclitus obscuripennis. 177. Adialytus ambiguus. 178. Adialytus salicaphis. 179. Adialytus thelaxis. 180. Adialytus veronicaecola. 181. Aphidius absinthii. 182. Aphidius arvensis. 183. Aphidius asteris. 184. Aphidius avenae. 185. Aphidius banksae. 186. Aphidius cingulatus. 187. Aphidius colemani. 188. Aphidius eadyi. 189. Aphidius eglanteriae. 190. Aphidius ervi. 191. Aphidius funebris. 

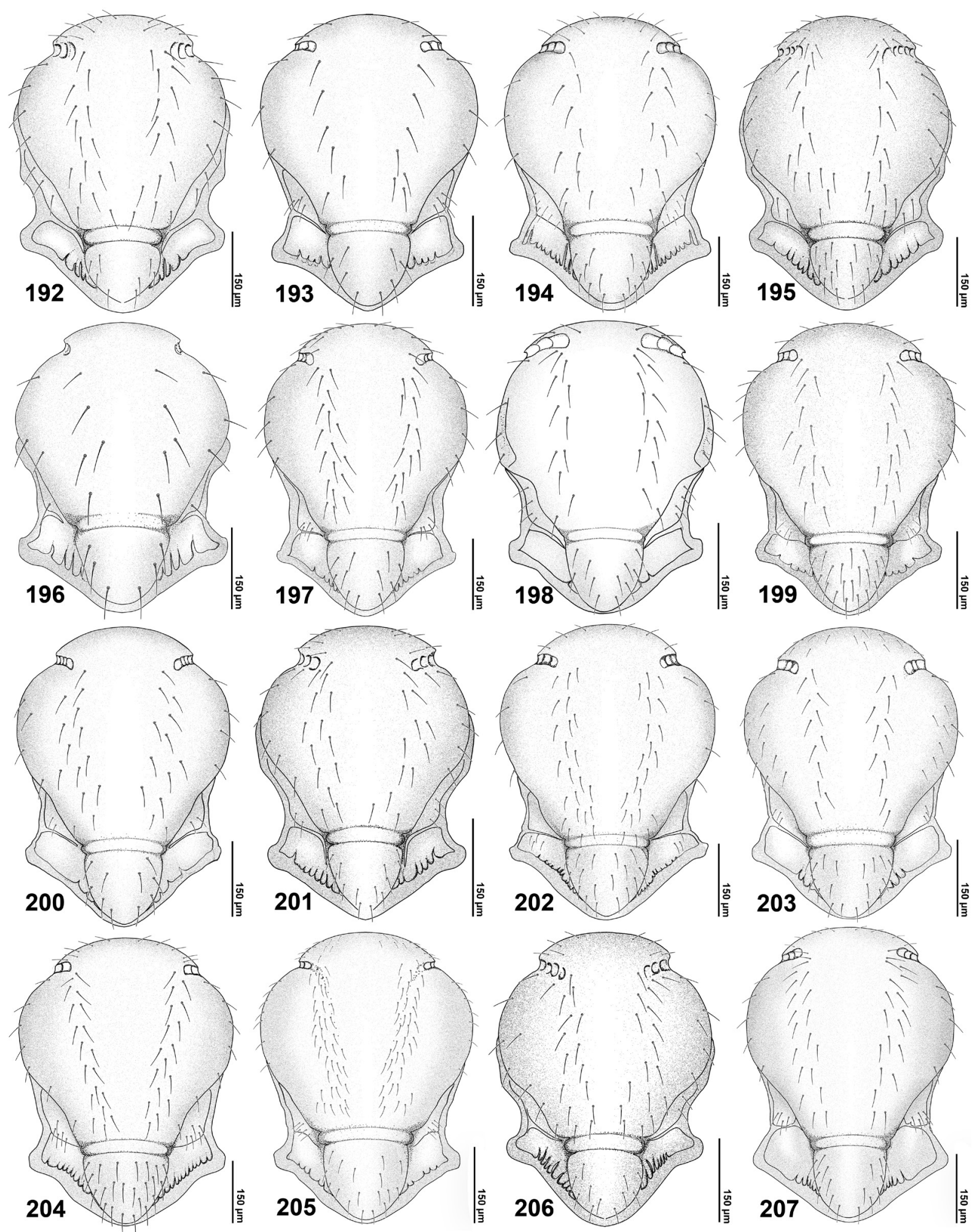

Figs 192-207. Dorsal aspect of mesonotum (†): 192. Aphidius hieraciorum. 193. Aphidius iranicus. 194. Aphidius matricariae. 195. Aphidius microlophii. 196. Aphidius myzocallidis. 197. Aphidius persicus. 198. Aphidius platensis. 199. Aphidius popovi. 200. Aphidius rhopalosiphi. 201. Aphidius ribis. 202. Aphidius rosae. 203. Aphidius salicis. 204. Aphidius setiger. 205. Aphidius smithi. 206. Aphidius sonchi. 207. Aphidius stigmaticus. 

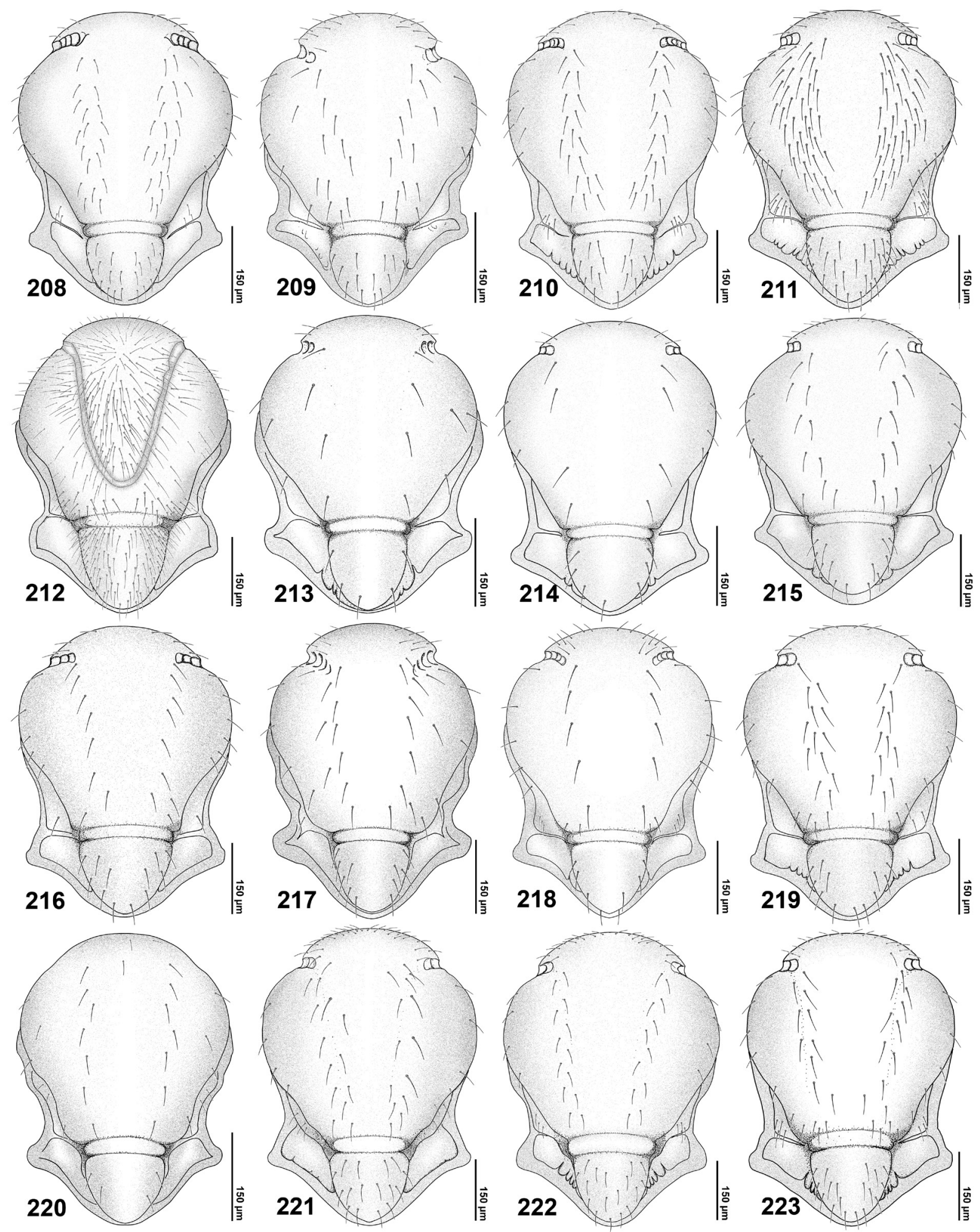

Figs 208-223. Dorsal aspect of mesonotum (†): 208. Aphidius transcaspicus. 209. Aphidius uroleuci. 210. Aphidius urticae. 211. Aphidius uzbekistanicus. 212. Areopraon lepelleyi. 213. Betuloxys hortorum. 214. Binodoxys acalephae. 215. Binodoxys angelicae. 216. Binodoxys brevicornis. 217. Binodoxys centaureae. 218. Binodoxys heraclei. 219. Diaeretiella rapae. 220. Diaeretus leucopterus. 221. Ephedrus cerasicola. 222. Ephedrus chaitophori. 223. Ephedrus helleni. 

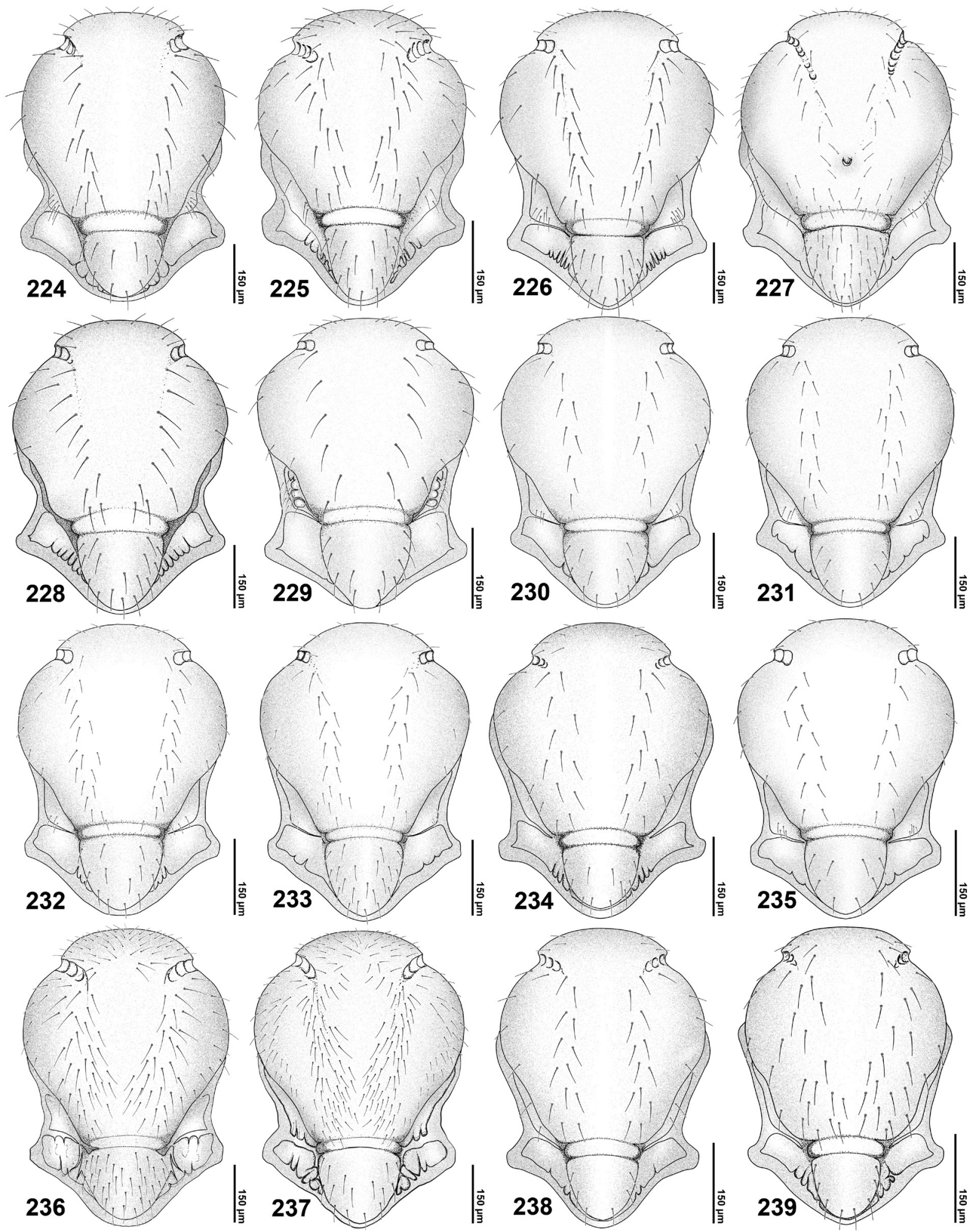

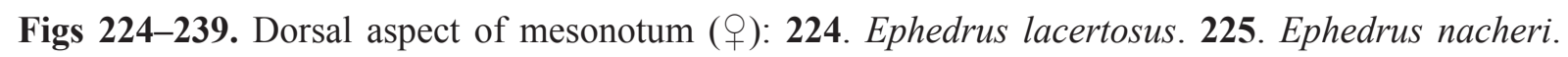
226. Ephedrus niger. 227. Ephedrus persicae. 228. Ephedrus plagiator. 229. Lipolexis gracilis. 230. Lysiphlebus cardui. 231. Lysiphlebus confusus. 232. Lysiphlebus desertorum. 233. Lysiphlebus fabarum. 234. Lysiphlebus fritzmuelleri. 235. Lysiphlebus testaceipes. 236. Monoctonia pistaciaecola. 237. Monoctonia vesicarii. 238. Monoctonus crepidis. 239. Monoctonus mali. 

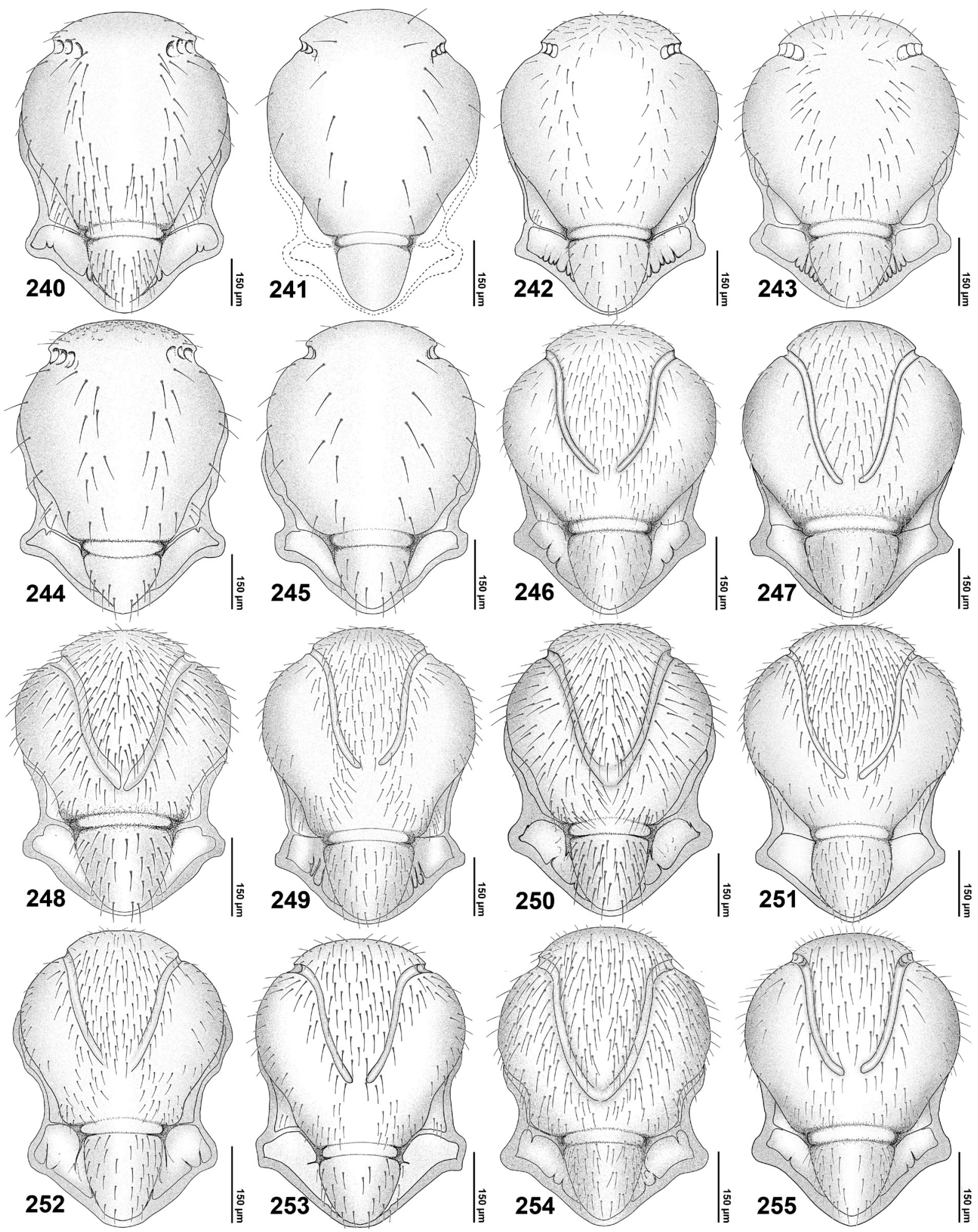

Figs 240-255. Dorsal aspect of mesonotum (P): 240. Pauesia abietis. 241. Pauesia anatolica. 242. Pauesia antennata. 243. Pauesia hazratbalensis. 244. Pauesia picta. 245. Pauesia silana. 246. Praon abjectum. 247. Praon absinthii. 248. Praon athenaeum. 249. Praon barbatum. 250. Praon bicolor. 251. Praon exsoletum. 252. Praon flavinode. 253. Praon gallicum. 254. Praon longicorne. 255. Praon necans. 

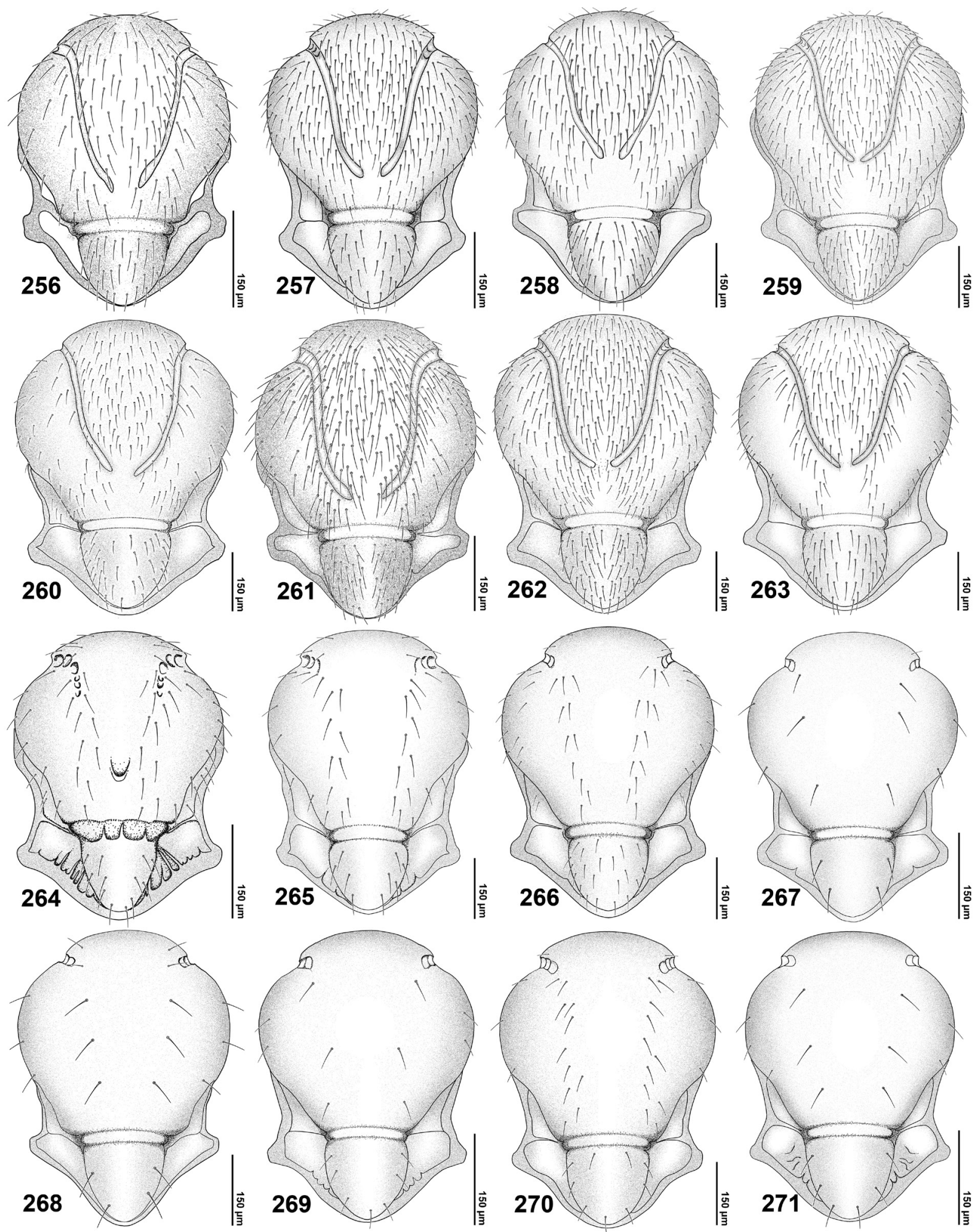

Figs 256-271. Dorsal aspect of mesonotum (†): 256. Praon nonveilleri. 257. Praon orpheusi. 258. Praon pubescens. 259. Praon rosaecola. 260. Praon unitum. 261. Praon uroleucon. 262. Praon volucre. 263. Praon yomenae. 264. Toxares deltiger. 265. Trioxys asiaticus. 266. Trioxys cirsii. 267. Trioxys complanatus. 268. Trioxys metacarpalis. 269. Trioxys pallidus. 270. Trioxys pannonicus. 271. Trioxys tanaceticola. 



Figs 272-286. Dorsal aspect of propodeum ( $($ ): 272. Aclitus obscuripennis. 273. Adialytus ambiguus. 274. Adialytus salicaphis. 275. Adialytus thelaxis. 276. Adialytus veronicaecola. 277. Aphidius absinthii. 278. Aphidius arvensis. 279. Aphidius asteris. 280. Aphidius avenae. 281. Aphidius banksae. 282. Aphidius cingulatus. 283. Aphidius colemani. 284. Aphidius eglanteriae. 285. Aphidius eadyi. 286. Aphidius ervi. 



293
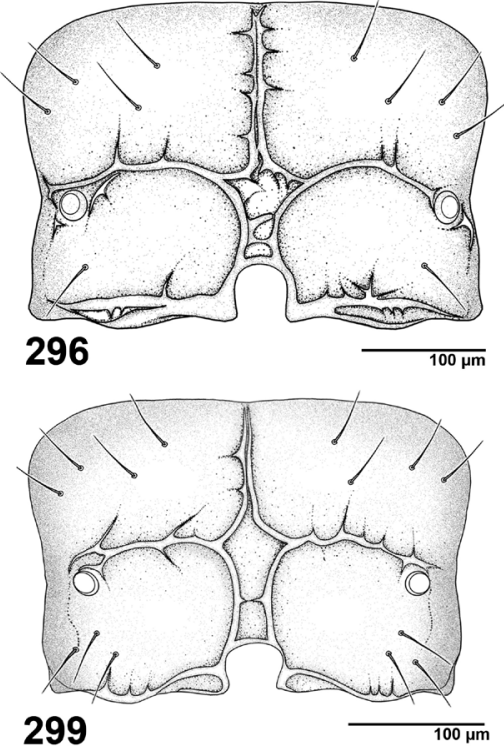
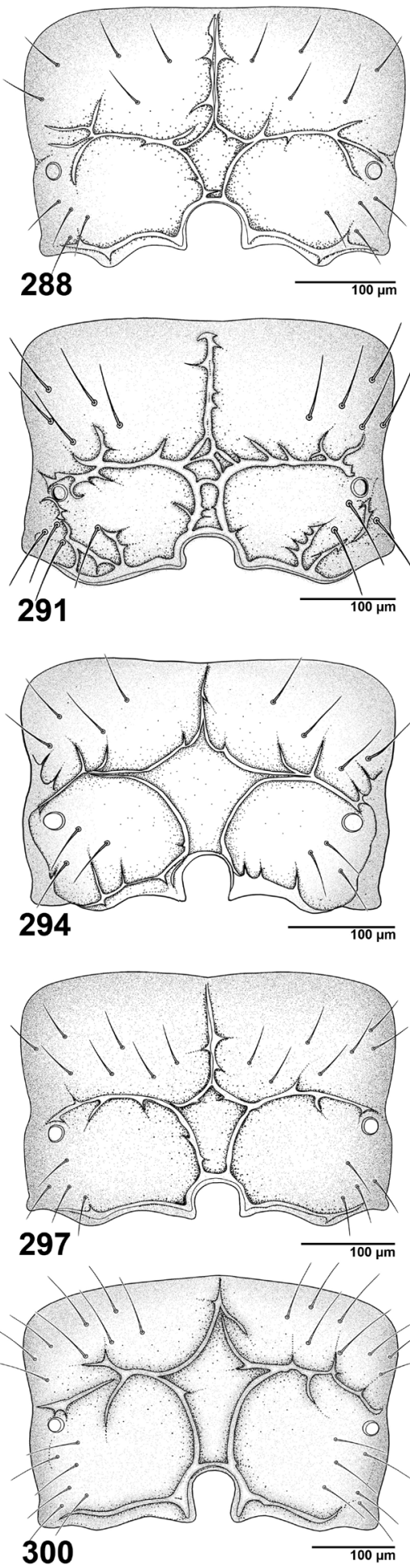


298

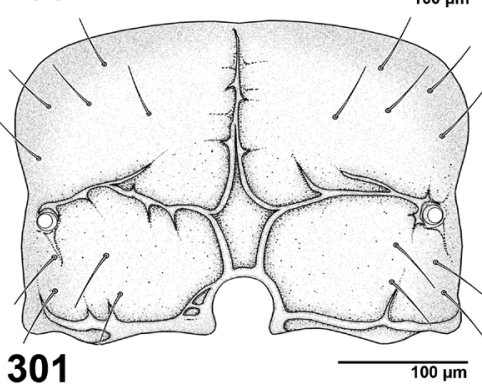

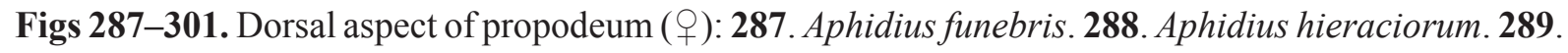
Aphidius iranicus. 290. Aphidius matricariae. 291. Aphidius microlophii. 292. Aphidius myzocallidis. 293. Aphidius persicus. 294. Aphidius platensis. 295. Aphidius popovi. 296. Aphidius rhopalosiphi. 297. Aphidius ribis. 298. Aphidius rosae. 299. Aphidius salicis. 300. Aphidius setiger. 301. Aphidius smithi. 

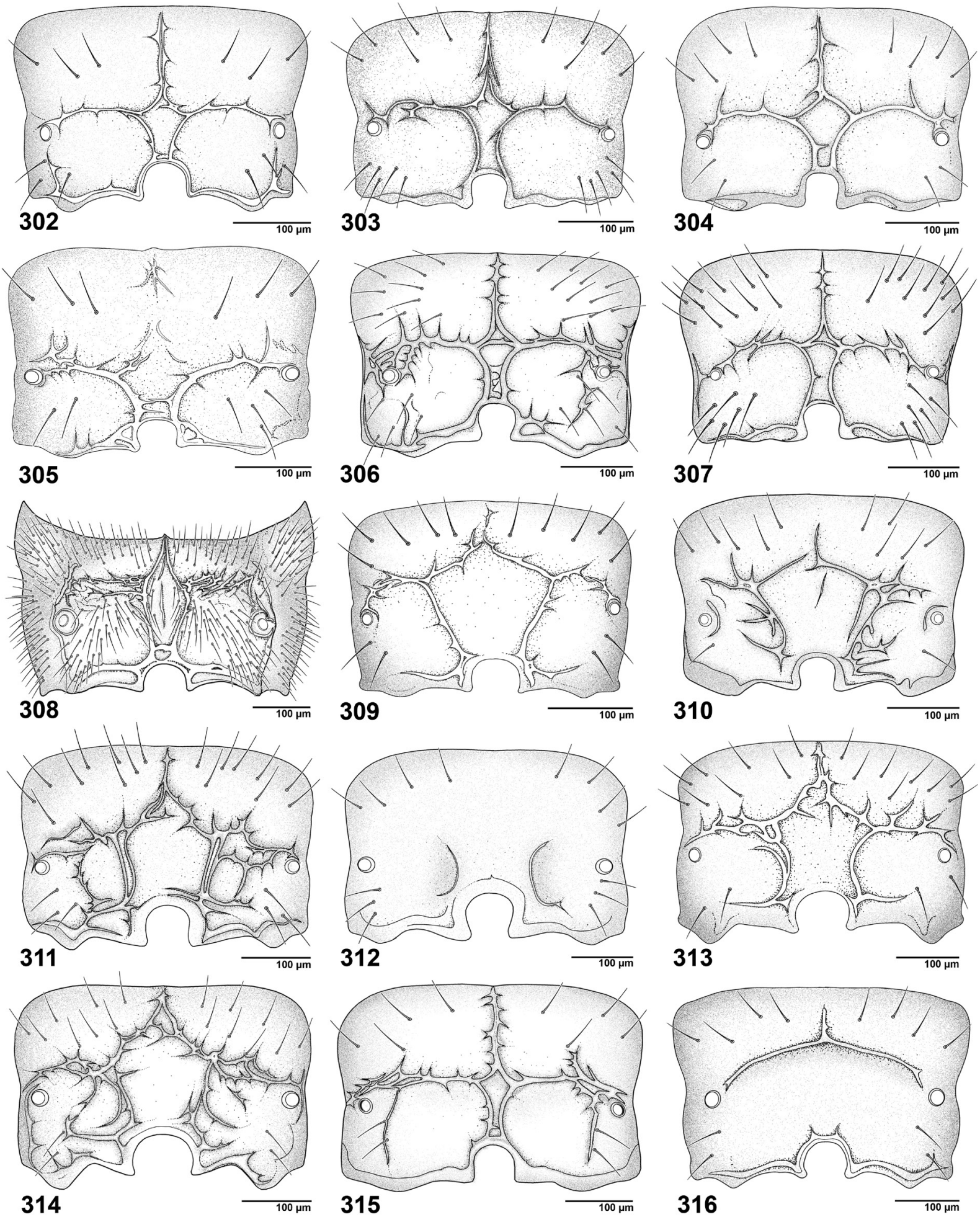

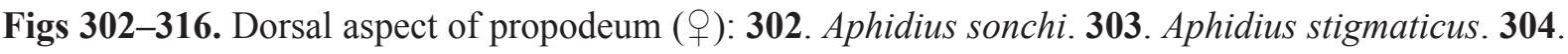
Aphidius transcaspicus. 305. Aphidius uroleuci. 306. Aphidius urticae. 307. Aphidius uzbekistanicus. 308. Areopraon lepelleyi.309. Betuloxys hortorum. 310. Binodoxys acalephae. 311. Binodoxys angelicae. 312. Binodoxys brevicornis. 313. Binodoxys centaureae. 314. Binodoxys heraclei. 315. Diaeretiella rapae. 316. Diaeretus leucopterus. 

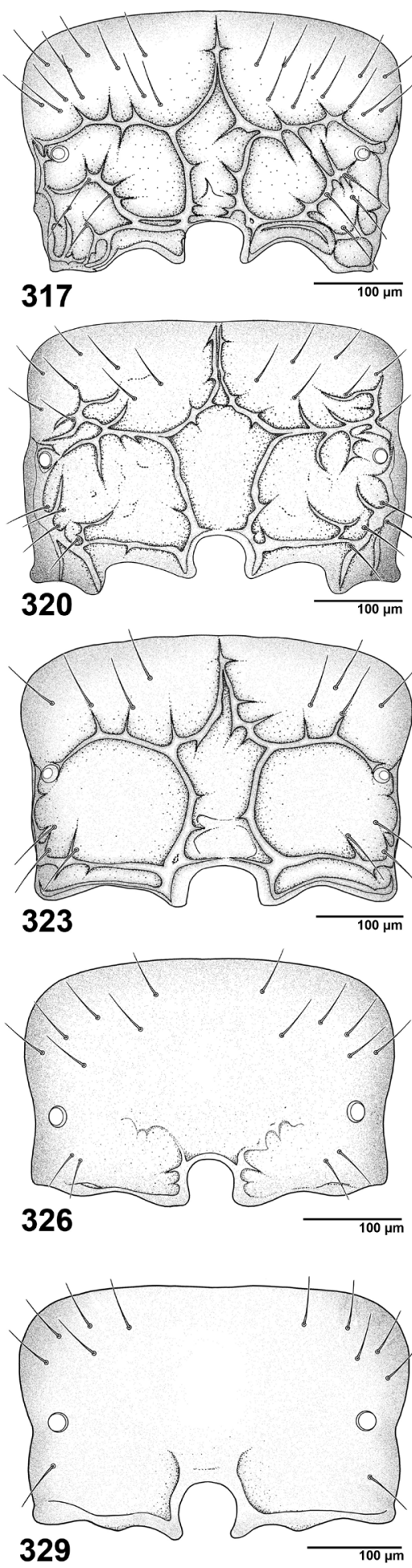
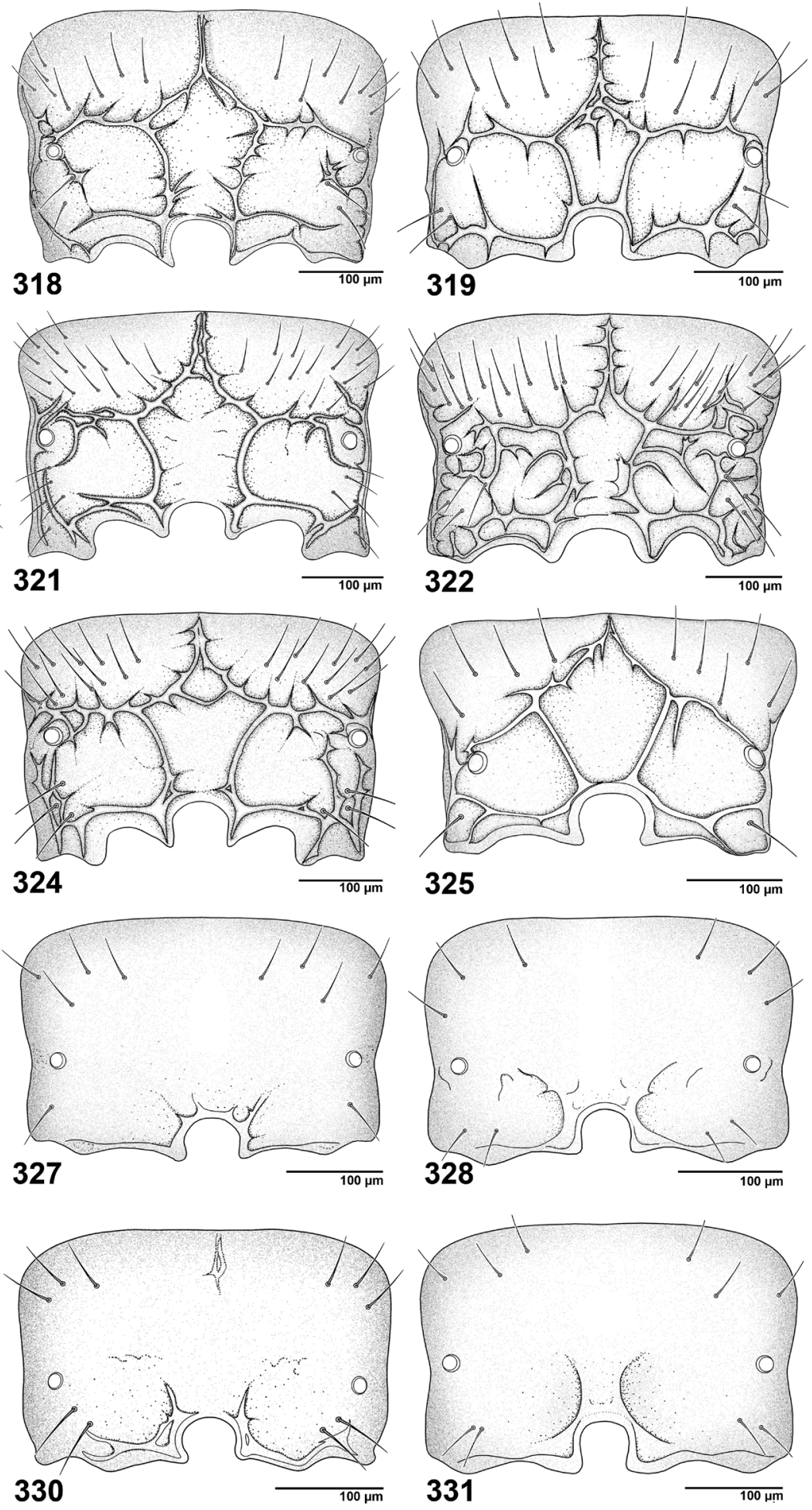

Figs 317-331. Dorsal aspect of propodeum (q): 317. Ephedrus cerasicola. 318. Ephedrus chaitophori. 319. Ephedrus helleni. 320. Ephedrus lacertosus. 321. Ephedrus nacheri. 322. Ephedrus niger. 323. Ephedrus persicae. 324. Ephedrus plagiator. 325. Lipolexis gracilis. 326. Lysiphlebus cardui. 327. Lysiphlebus confusus. 328. Lysiphlebus desertorum. 329. Lysiphlebus fabarum. 330. Lysiphlebus fritzmuelleri. 331. Lysiphlebus testaceipes. 

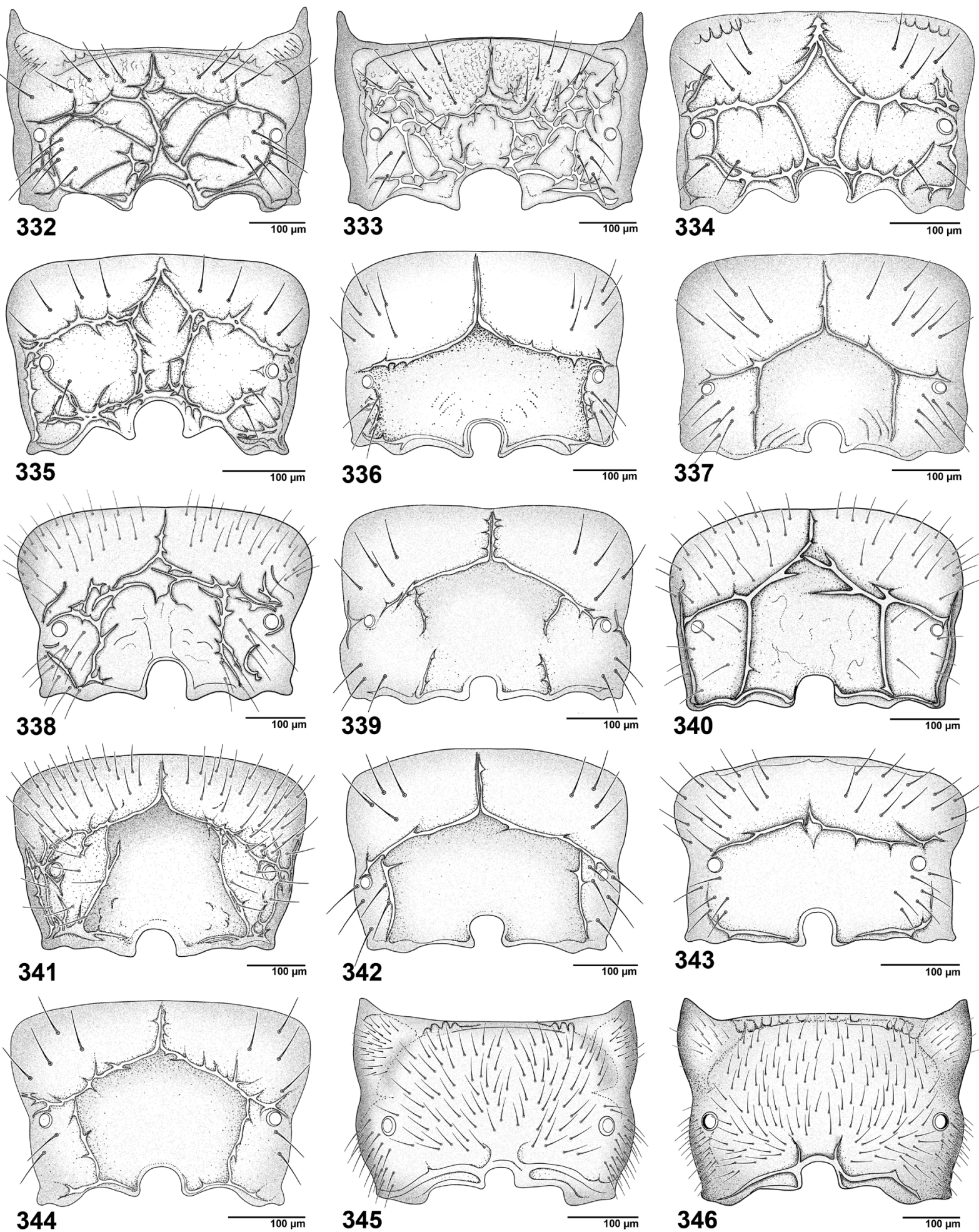

Figs 332-346. Dorsal aspect of propodeum (†): 332. Monoctonia pistaciaecola. 333. Monoctonia vesicarii. 334. Monoctonus crepidis. 335. Monoctonus mali. 336. Pauesia abietis. 337. Pauesia anatolica. 338. Pauesia antennata. 339. Pauesia cedrobii. 340. Pauesia hazratbalensis. 341. Pauesia picta. 342. Pauesia pini. 343. Pauesia silana. 344. Pauesia unilachni. 345. Praon abjectum. 346. Praon absinthii. 

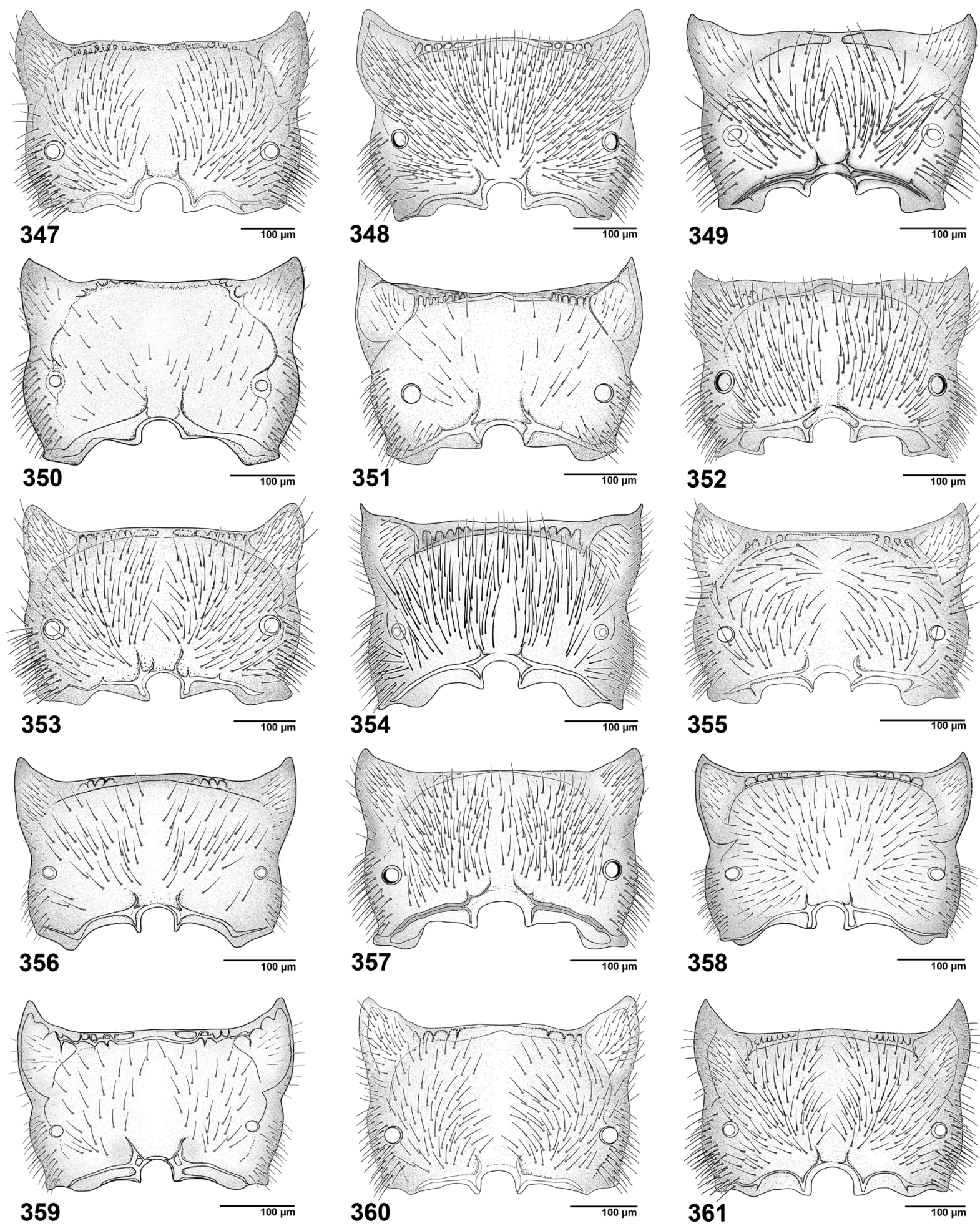

Figs 347-361. Dorsal aspect of propodeum (†): 347. Praon athenaeum. 348. Praon barbatum. 349. Praon bicolor. 350. Praon exsoletum. 351. Praonflavinode. 352. Praon gallicum. 353. Praon longicorne. 354. Praon necans. 355. Praon nonveilleri. 356. Praon orpheusi. 357. Praon pubescens. 358. Praon rosaecola. 359. Praon unitum. 360. Praon uroleucon. 361. Praon volucre. 



Figs 362-373. Dorsal aspect of propodeum ( $($ ) : 362. Praon yomenae. 363. Toxares deltiger. 364. Trioxys asiaticus. 365. Trioxys cirsii. 366. Trioxys complanatus. 367. Trioxys curvicaudus. 368. Trioxys metacarpalis. 369. Trioxys moshei. 370. Trioxys pallidus. 371. Trioxys pannonicus. 372. Trioxys quercicola. 373. Trioxys tanaceticola. 

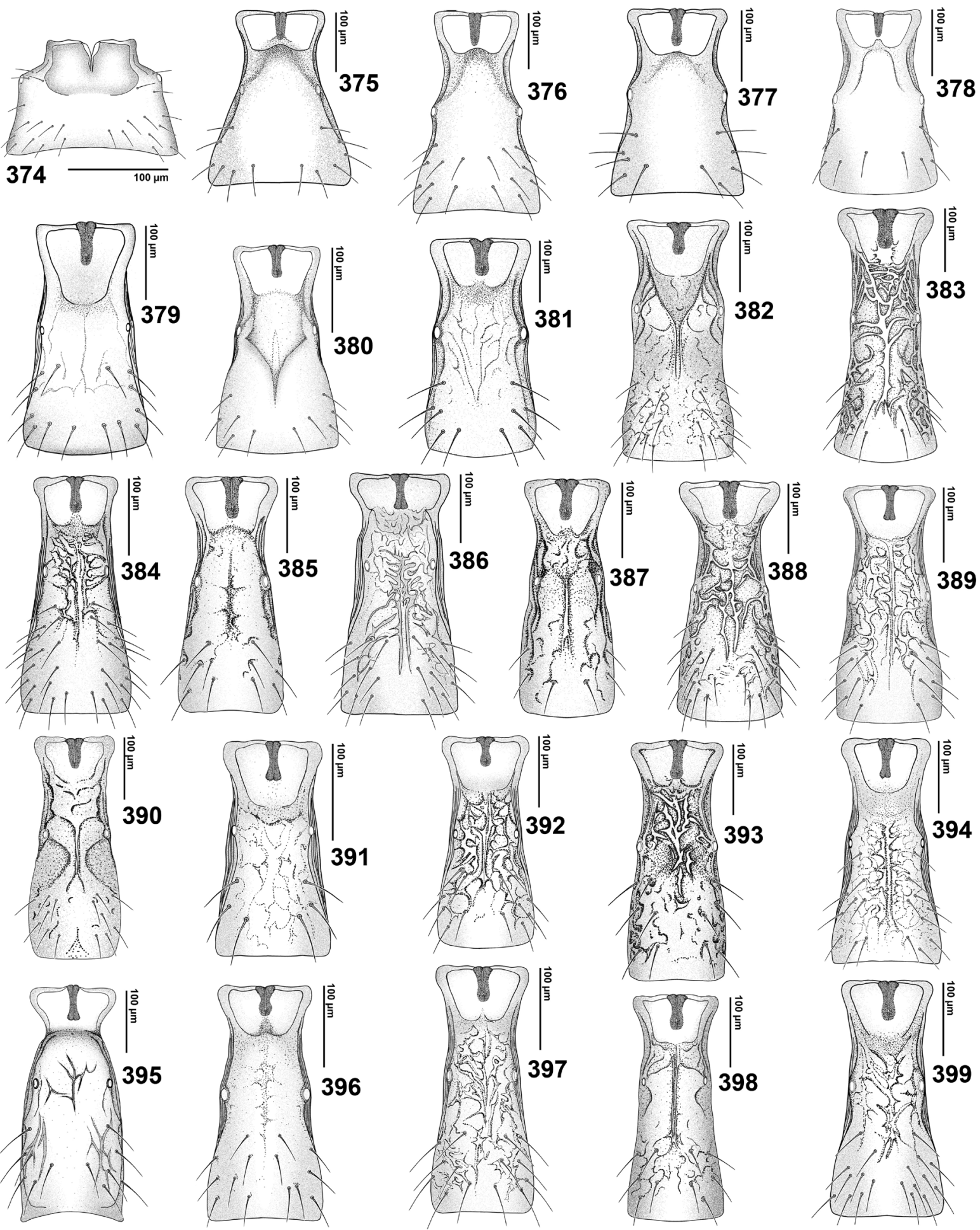

Figs 374-399. Dorsal aspect of petiole (†): 374. Aclitus obscuripennis. 375. Adialytus ambiguus. 376. Adialytus salicaphis. 377. Adialytus thelaxis. 378. Adialytus veronicaecola. 379. Aphidius absinthii. $\mathbf{3 8 0}$. Aphidius arvensis. 381. Aphidius asteris. 382. Aphidius avenae. 383. Aphidius banksae. 384. Aphidius cingulatus. 385. Aphidius colemani. 386. Aphidius eadyi. 387. Aphidius eglanteriae. 388 Aphidius ervi. 389. Aphidius funebris. 390. Aphidius hieraciorum. 391. Aphidius iranicus. 392. Aphidius matricariae. 393. Aphidius microlophii. 394. Aphidius persicus. 395. Aphidius platensis. 396. Aphidius popovi. 397. Aphidius rhopalosiphi. 398. Aphidius ribis. 399. Aphidius rosae. 



Figs 400-424. Dorsal aspect of petiole (†): 400. Aphidius salicis. 401. Aphidius setiger. 402. Aphidius smithi. 403. Aphidius sonchi. 404. Aphidius stigmaticus. 405. Aphidius transcaspicus. 406. Aphidius uroleuci. 407. Aphidius urticae. 408. Aphidius uzbekistanicus. 409. Areopraon lepelleyi. 410. Betuloxys hortorum. 411. Binodoxys acalephae. 412. Binodoxys angelicae. 413. Binodoxys brevicornis. 414. Binodoxys centaureae. 415. Binodoxys heraclei.416. Diaeretiella rapae. 417. Diaeretus leucopterus. 418. Ephedrus cerasicola. 419. Ephedrus chaitophori. 420. Ephedrus helleni. 421. Ephedrus lacertosus. 422. Ephedrus nacheri. 423. Ephedrus niger. 424. Ephedrus persicae. 

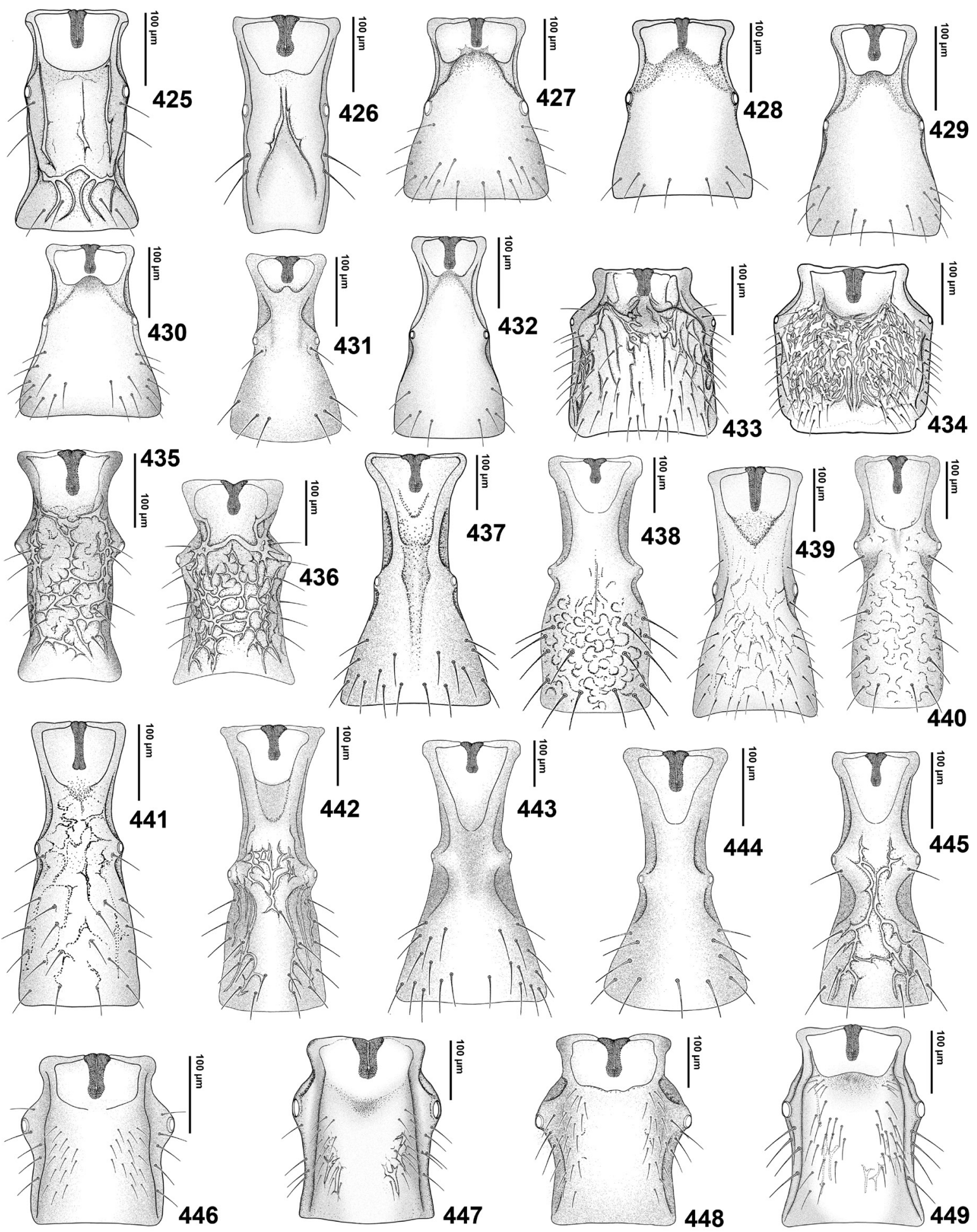

Figs 425-449. Dorsal aspect of petiole ( $($ ): 425. Ephedrus plagiator. 426. Lipolexis gracilis. 427. Lysiphlebus cardui. 428. Lysiphlebus confusus. 429. Lysiphlebus desertorum. 430. Lysiphlebus fabarum. 431. Lysiphlebus fritzmuelleri. 432. Lysiphlebus testaceipes. 433. Monoctonia pistaciaecola. 434. Monoctonia vesicarii. 435. Monoctonus crepidis. 436. Monoctonus mali. 437. Pauesia abietis. 438. Pauesia anatolica. 439. Pauesia antennata. 440. Pauesia cedrobii. 441. Pauesia hazratbalensis. 442. Pauesia picta. 443. Pauesia pini. 444. Pauesia silana. 445. Pauesia unilachni. 446. Praon abjectum. 447. Praon absinthii. 448. Praon athenaeum. 449. Praon barbatum. 

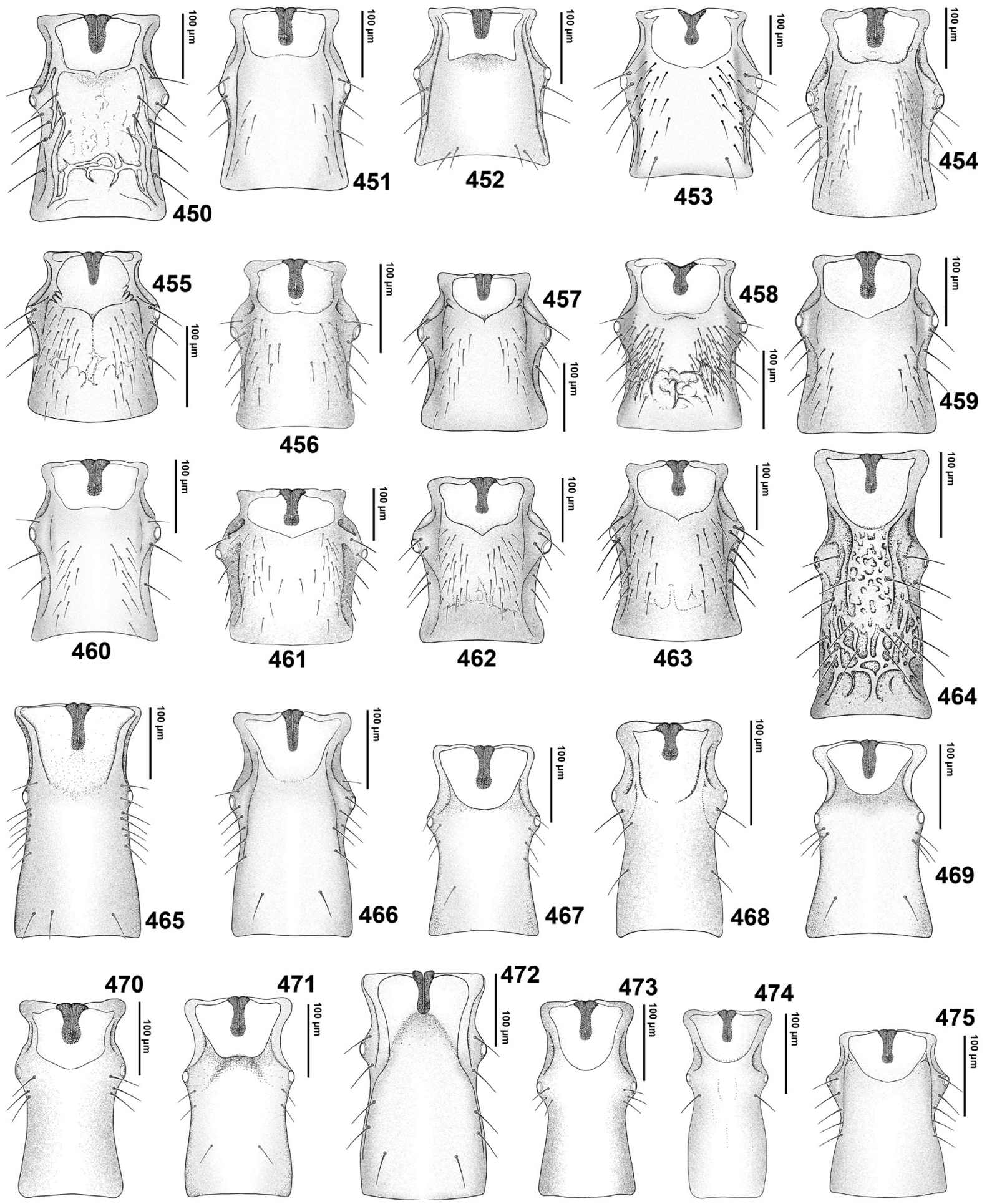

Figs 450-475. Dorsal aspect of petiole ( $($ ): 450. Praon bicolor. 451. Praon exsoletum. 452. Praon flavinode. 453. Praon gallicum. 454. Praon longicorne. 455. Praon necans. 456. Praon nonveilleri. 457. Praon orpheusi. 458. Praon pubescens. 459. Praon rosaecola. 460. Praon unitum. 461. Praon uroleucon. 462. Praon volucre. 463. Praon yomenae. 464. Toxares deltiger. 465. Trioxys asiaticus. 466. Trioxys cirsii. 467. Trioxys complanatus. 468. Trioxys curvicaudus. 469. Trioxys metacarpalis. 470. Trioxys moshei. 471. Trioxys pallidus. 472. Trioxys pannonicus. 473. Trioxys pappi. 474. Trioxys quercicola. 475. Trioxys tanaceticola. 

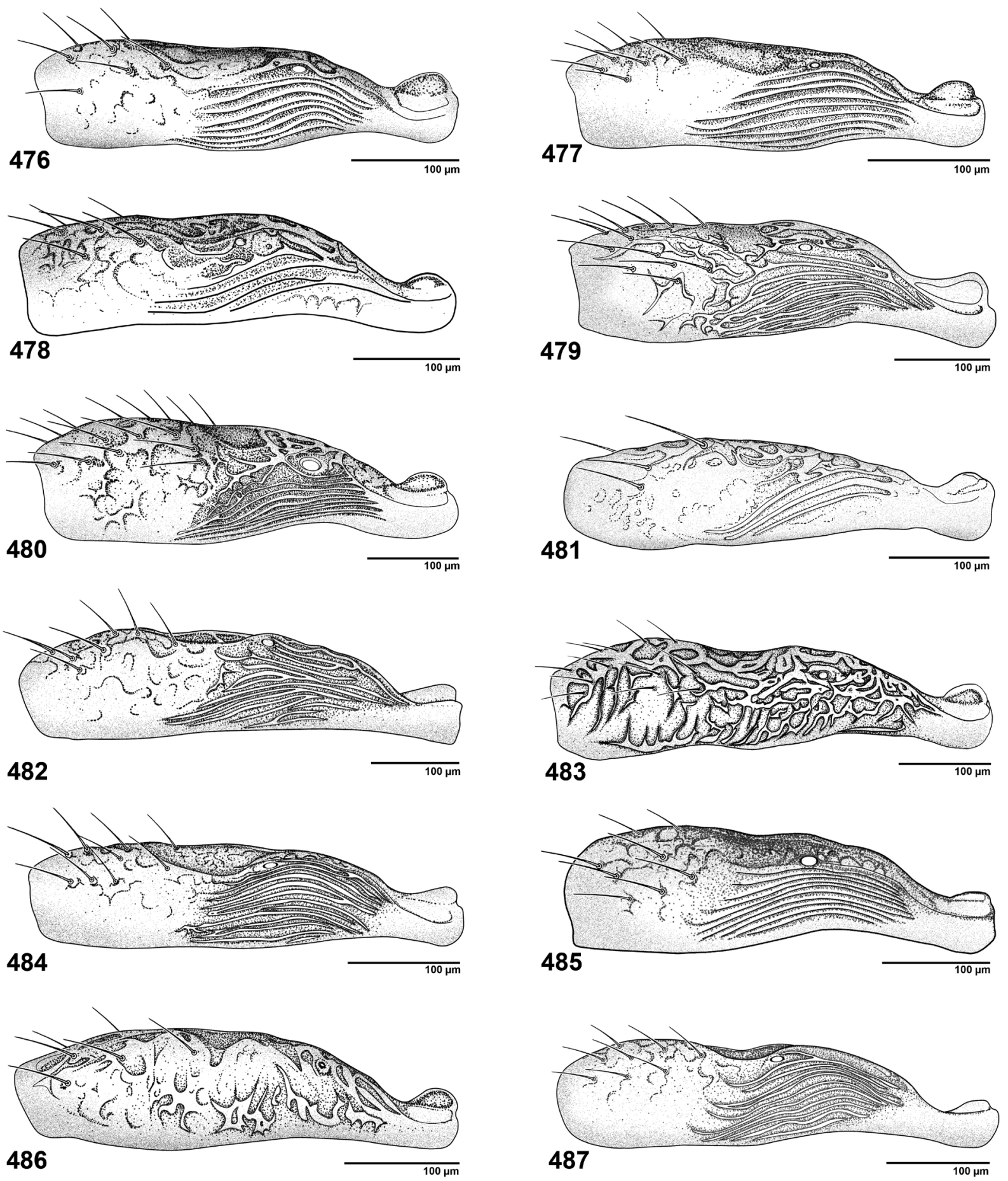

Figs 476-487. Anterolateral aspect of petiole in Aphidius species (†): 476. Aphidius absinthii. 477. Aphidius asteris. 478. Aphidius avenae. 479. Aphidius banksae. 480. Aphidius cingulatus. 481. Aphidius colemani. 482. Aphidius eadyi. 483. Aphidius ervi. 484. Aphidius funebris. 485. Aphidius matricariae. 486. Aphidius microlophii. 487. Aphidius persicus. 

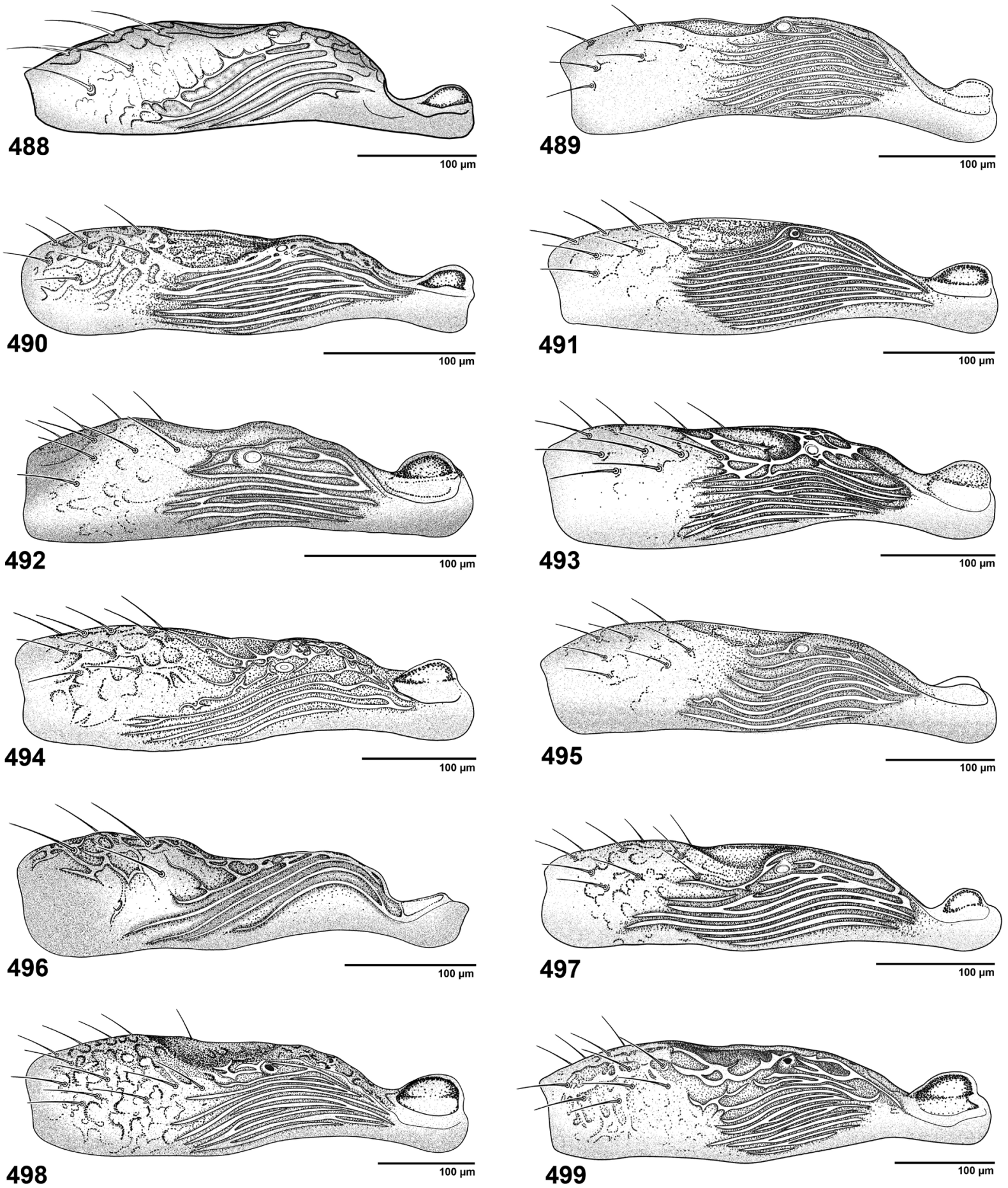

Figs 488-499. Anterolateral aspect of petiole in Aphidius species (†): 488. Aphidius platensis. 489. Aphidius popovi. 490. Aphidius rhopalosiphi. 491. Aphidius rosae. 492. Aphidius salicis. 493. Aphidius setiger. 494. Aphidius smithi. 495. Aphidius stigmaticus. 496. Aphidius transcaspicus. 497. Aphidius uroleuci. 498. Aphidius urticae. 499. Aphidius uzbekistanicus. 

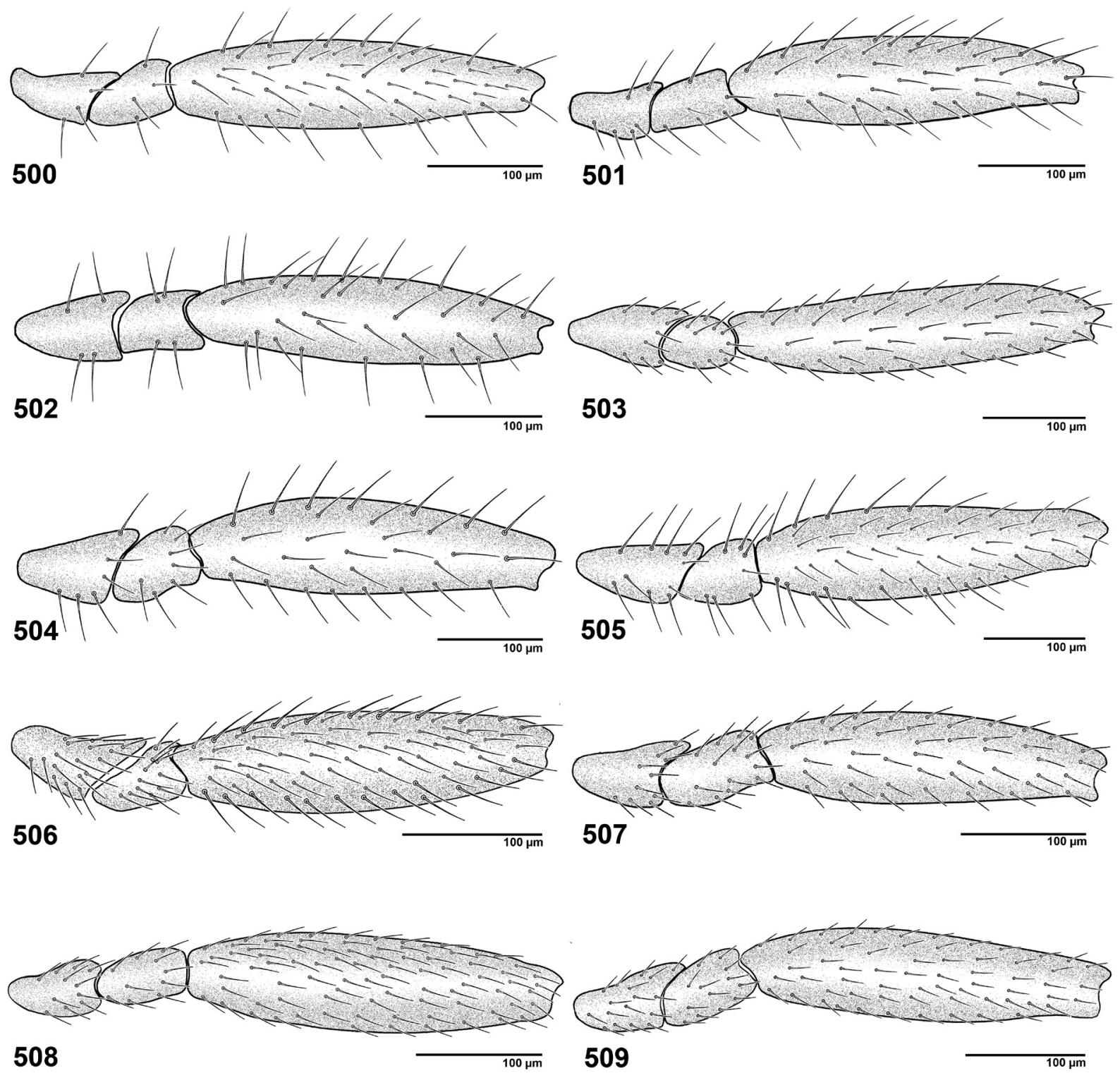

Figs 500-509. Lateral aspect of hind femur in Adialytus and Lysiphlebus species ( + ): 500. Adialytus ambiguus. 501. Adialytus salicaphis. 502. Adialytus thelaxis. 503. Adialytus veronicaecola. 504. Lysiphlebus cardui. 505. Lysiphlebus confusus. 506. Lysiphlebus desertorum. 507. Lysiphlebus fabarum. 508. Lysiphlebus fritzmuelleri. 509. Lysiphlebus testaceipes. 


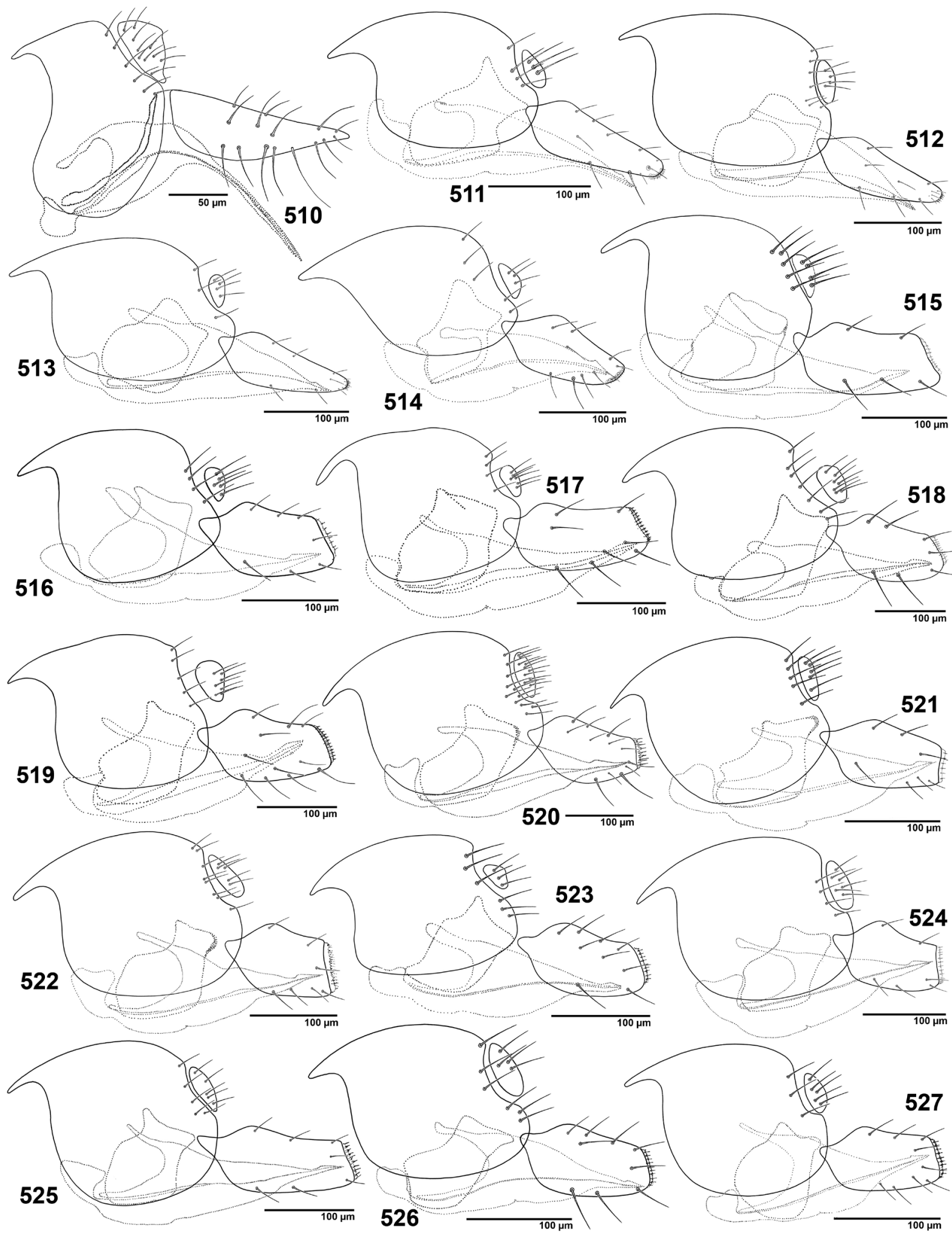

Figs 510-527. Lateral aspect of $q$ genitalia: 510. Aclitus obscuripennis. 511. Adialytus ambiguus. 512. Adialytus salicaphis. 513. Adialytus thelaxis. 514. Adialytus veronicaecola. 515. Aphidius absinthii. 516. Aphidius arvensis. 517. Aphidius asteris. 518. Aphidius avenae. 519. Aphidius banksae. 520. Aphidius cingulatus. 521. Aphidius colemani. 522. Aphidius eadyi. 523. Aphidius eglanteriae. 524. Aphidius ervi. 525. Aphidius funebris. 526. Aphidius hieraciorum. 527. Aphidius iranicus. 


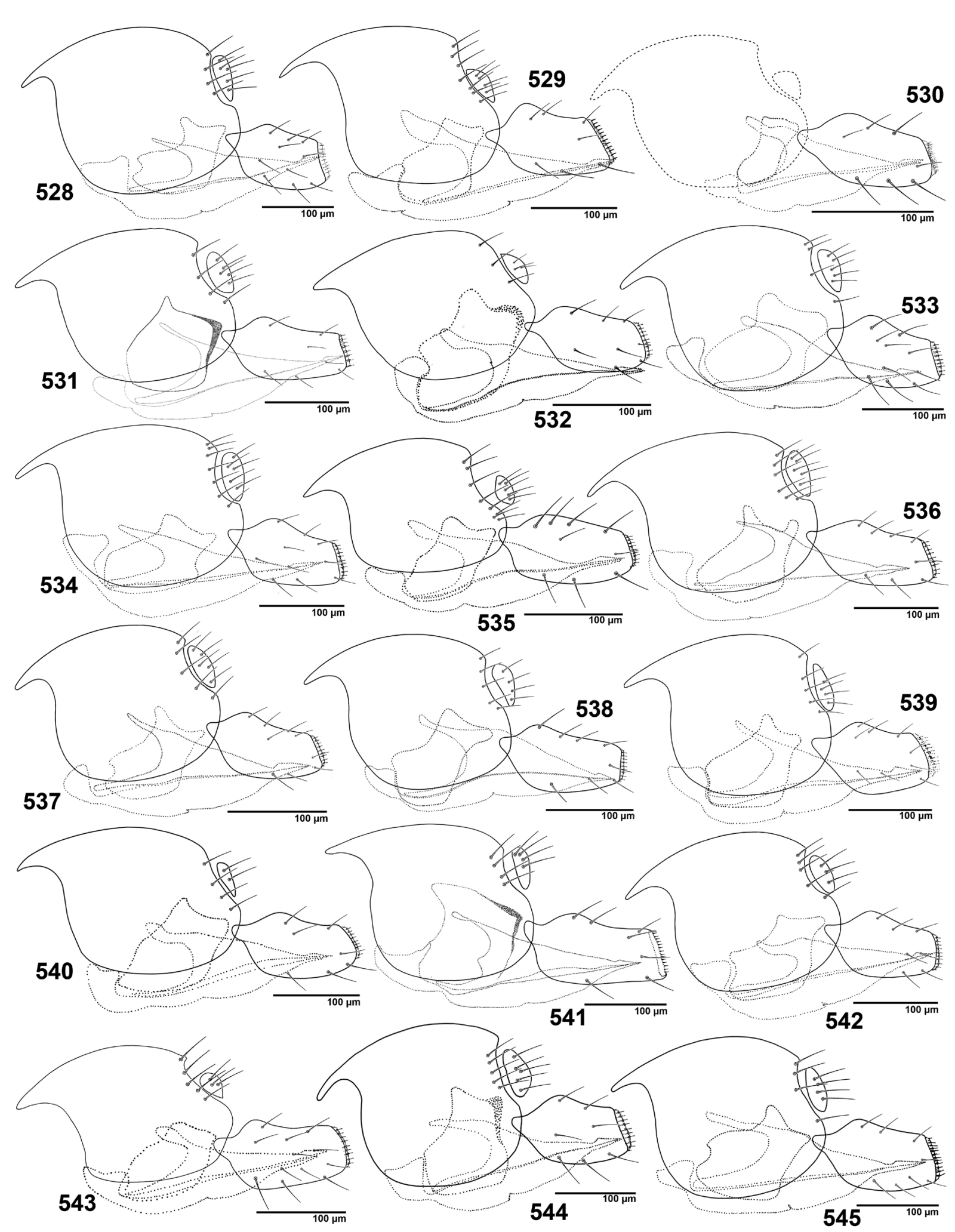

Figs 528-545. Lateral aspect of $q$ genitalia: 528. Aphidius matricariae. 529. Aphidius microlophii. 530. Aphidius myzocallidis. 531. Aphidius persicus. 532. Aphidius platensis. 533. Aphidius popovi. 534. Aphidius rhopalosiphi. 535. Aphidius ribis. 536. Aphidius rosae. 537. Aphidius salicis. 538. Aphidius setiger. 539. Aphidius smithi. 540. Aphidius sonchi. 541. Aphidius stigmaticus. 542. Aphidius transcaspicus. 543. Aphidius uroleuci. 544. Aphidius urticae. 545. Aphidius uzbekistanicus. 




Figs 546-562. Lateral aspect of $q$ genitalia: 546. Areopraon lepelleyi. 547. Betuloxys hortorum. 548. Binodoxys acalephae. 549. Binodoxys angelicae. 550. Binodoxys brevicornis. 551. Binodoxys centaureae. 552. Binodoxys heraclei. 553. Diaeretiella rapae. 554. Diaeretus leucopterus. 555. Ephedrus cerasicola. 556. Ephedrus chaitophori. 557. Ephedrus helleni. 558. Ephedrus lacertosus. 559. Ephedrus nacheri. 560. Ephedrus niger. 561. Ephedrus persicae. 562. Ephedrus plagiator. 


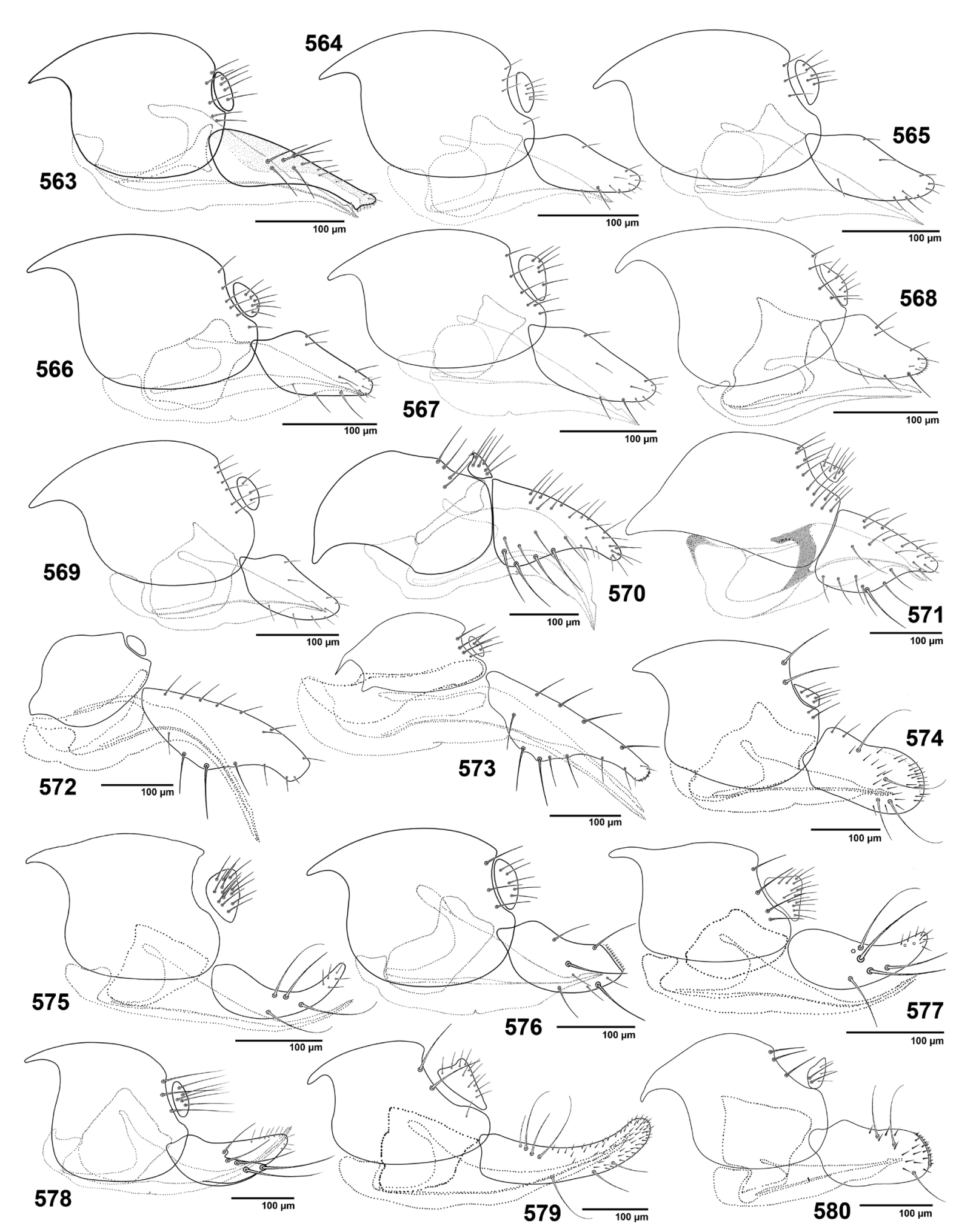

Figs 563-580. Lateral aspect of $q$ genitalia: 563. Lipolexis gracilis. 564. Lysiphlebus cardui. $\mathbf{5 6 5}$. Lysiphlebus confusus. 566. Lysiphlebus desertorum. 567. Lysiphlebus fabarum. 568. Lysiphlebus fritzmuelleri. 569. Lysiphlebus testaceipes. 570. Monoctonia pistaciaecola. 571. Monoctonia vesicarii. 572. Monoctonus crepidis. 573. Monoctonus mali. 574. Pauesia abietis. 575. Pauesia anatolica. 576. Pauesia antennata. 577. Pauesia cedrobii. 578. Pauesia hazratbalensis. 579. Pauesia picta. 580. Pauesia pini. 


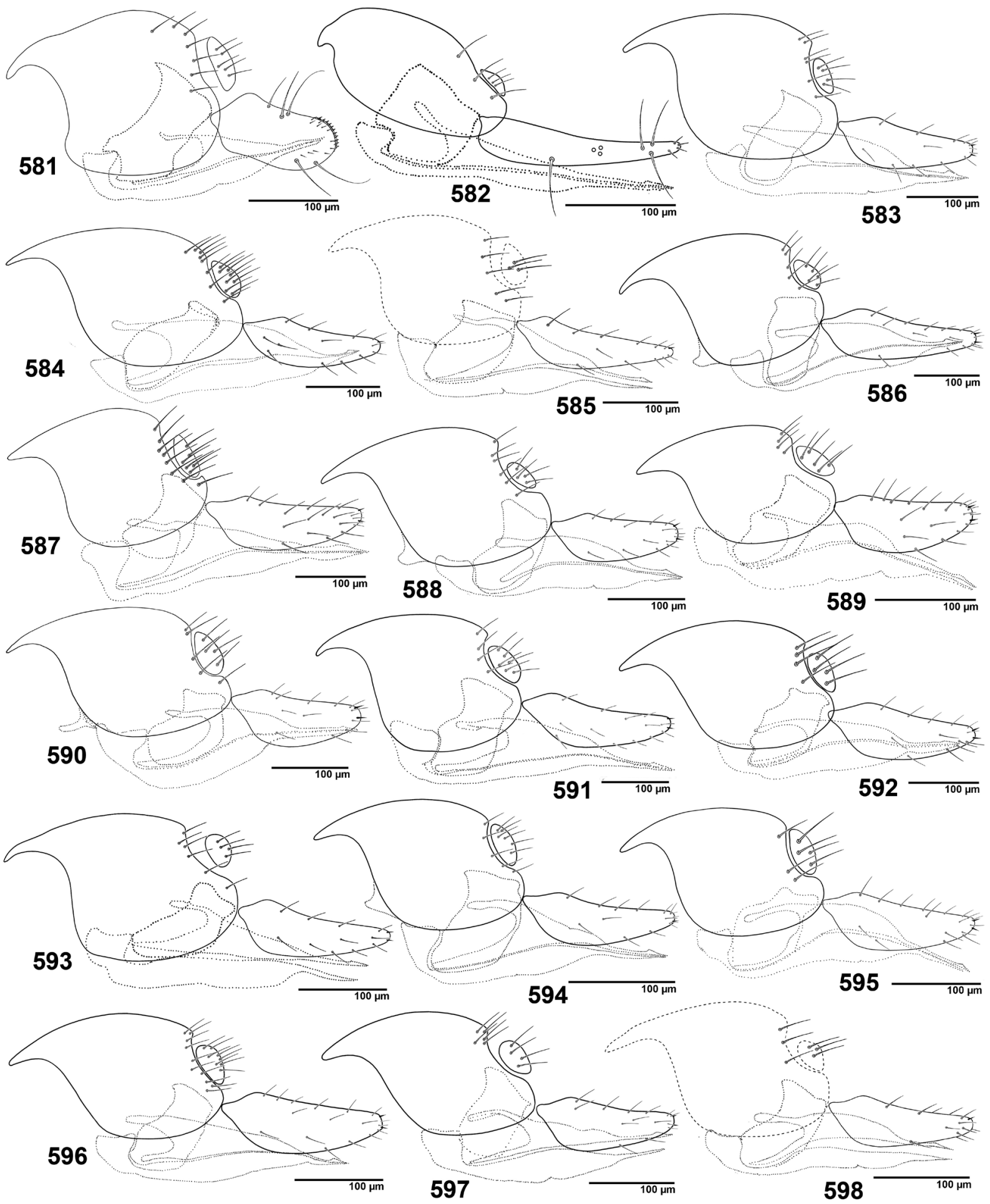

Figs 581-598. Lateral aspect of $q$ genitalia: 581. Pauesia silana. 582. Pauesia unilachni. 583. Praon abjectum. 584. Praon absinthii. 585. Praon athenaeum. 586. Praon barbatum. 587. Praon bicolor. 588. Praon exsoletum. 589. Praon flavinode. 590. Praon gallicum. 591. Praon longicorne. 592. Praon necans. 593. Praon nonveilleri. 594. Praon orpheusi. 595. Praon pubescens. 596. Praon rosaecola. 597. Praon unitum. 598. Praon uroleucon. 


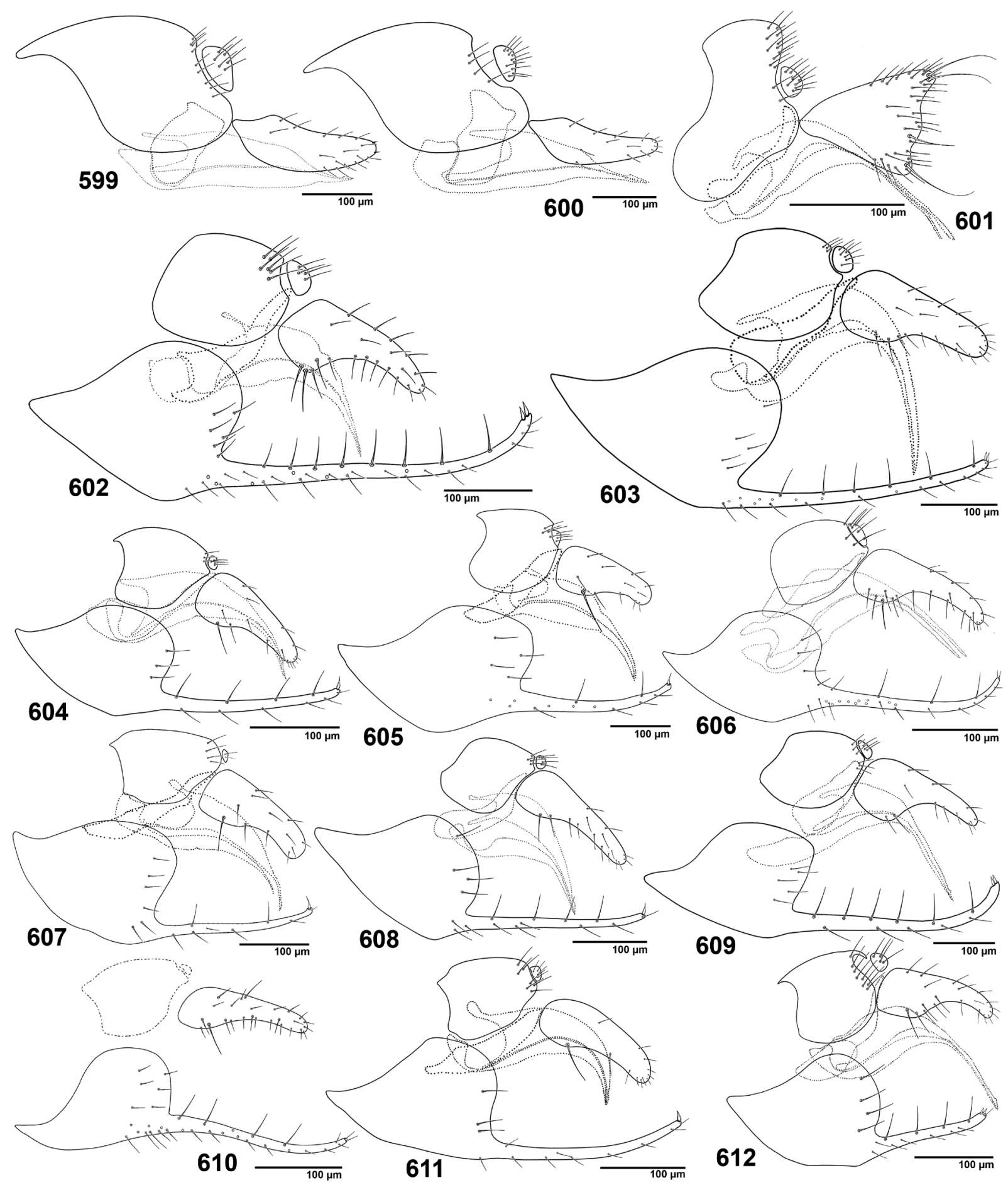

Figs 599-612. Lateral aspect of $q$ genitalia: 599. Praon volucre. 600. Praon yomenae. 601. Toxares deltiger. 602. Trioxys asiaticus. 603. Trioxys cirsii. 604. Trioxys complanatus. 605. Trioxys curvicaudus. 606. Trioxys metacarpalis. 607. Trioxys moshei. 608. Trioxys pallidus. 609. Trioxys pannonicus. 610. Trioxys pappi. 611. Trioxys quercicola. 612. Trioxys tanaceticola. 


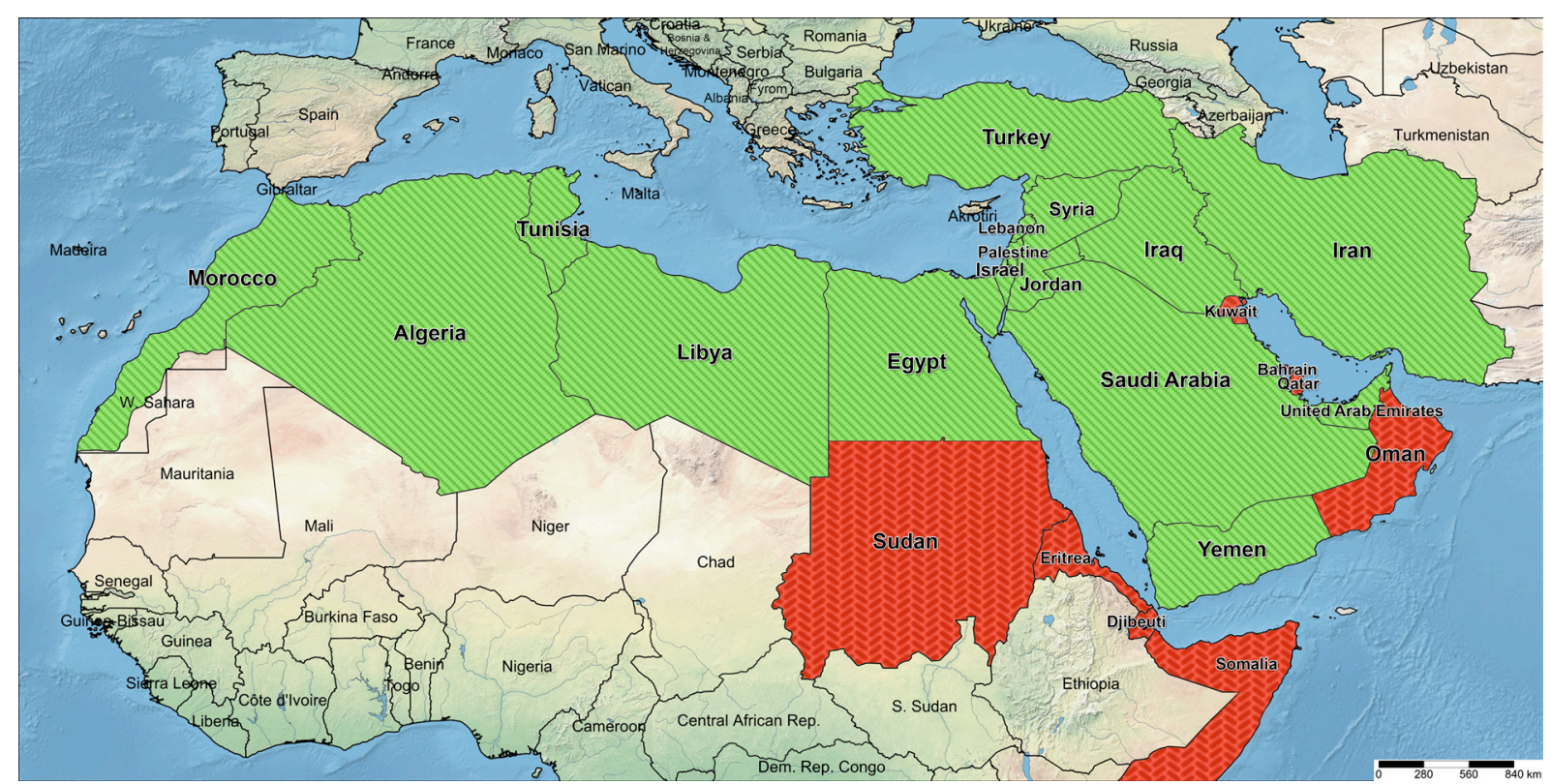

Fig. 613. Map of the Middle East and North Africa. A country is highlighted with green color indicates that there are available data on aphidiine parasitoids. A country highlighted with red color, indicates that there are no available data on aphidiine parasitoids 